\title{
Diagnostic, neuropsychiatric and therapeutic considerations in epilepsy and intellectual disability
}

Citation for published version (APA):

Snoeijen-Schouwenaars, F. (2019). Diagnostic, neuropsychiatric and therapeutic considerations in epilepsy and intellectual disability: medical perspectives. [Doctoral Thesis, Maastricht University]. Ridderprint BV. https://doi.org/10.26481/dis.20191009fs

Document status and date:

Published: 01/01/2019

DOI:

10.26481/dis.20191009fs

Document Version:

Publisher's PDF, also known as Version of record

\section{Please check the document version of this publication:}

- A submitted manuscript is the version of the article upon submission and before peer-review. There can be important differences between the submitted version and the official published version of record.

People interested in the research are advised to contact the author for the final version of the publication, or visit the DOI to the publisher's website.

- The final author version and the galley proof are versions of the publication after peer review.

- The final published version features the final layout of the paper including the volume, issue and page numbers.

Link to publication

\footnotetext{
General rights rights.

- You may freely distribute the URL identifying the publication in the public portal. please follow below link for the End User Agreement:

www.umlib.nl/taverne-license

Take down policy

If you believe that this document breaches copyright please contact us at:

repository@maastrichtuniversity.nl

providing details and we will investigate your claim.
}

Copyright and moral rights for the publications made accessible in the public portal are retained by the authors and/or other copyright owners and it is a condition of accessing publications that users recognise and abide by the legal requirements associated with these

- Users may download and print one copy of any publication from the public portal for the purpose of private study or research.

- You may not further distribute the material or use it for any profit-making activity or commercial gain

If the publication is distributed under the terms of Article $25 \mathrm{fa}$ of the Dutch Copyright Act, indicated by the "Taverne" license above, 


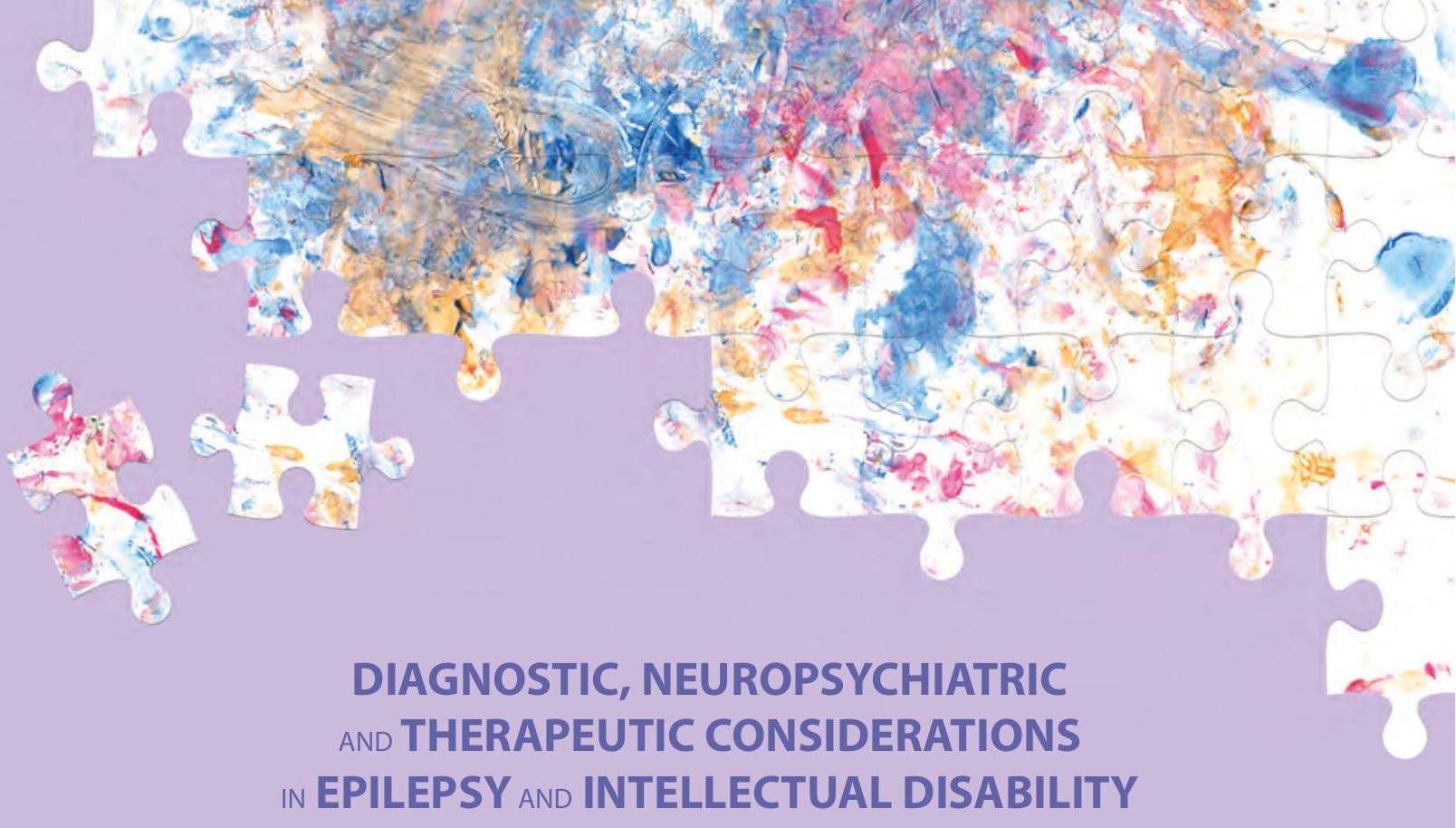

medical perspectives

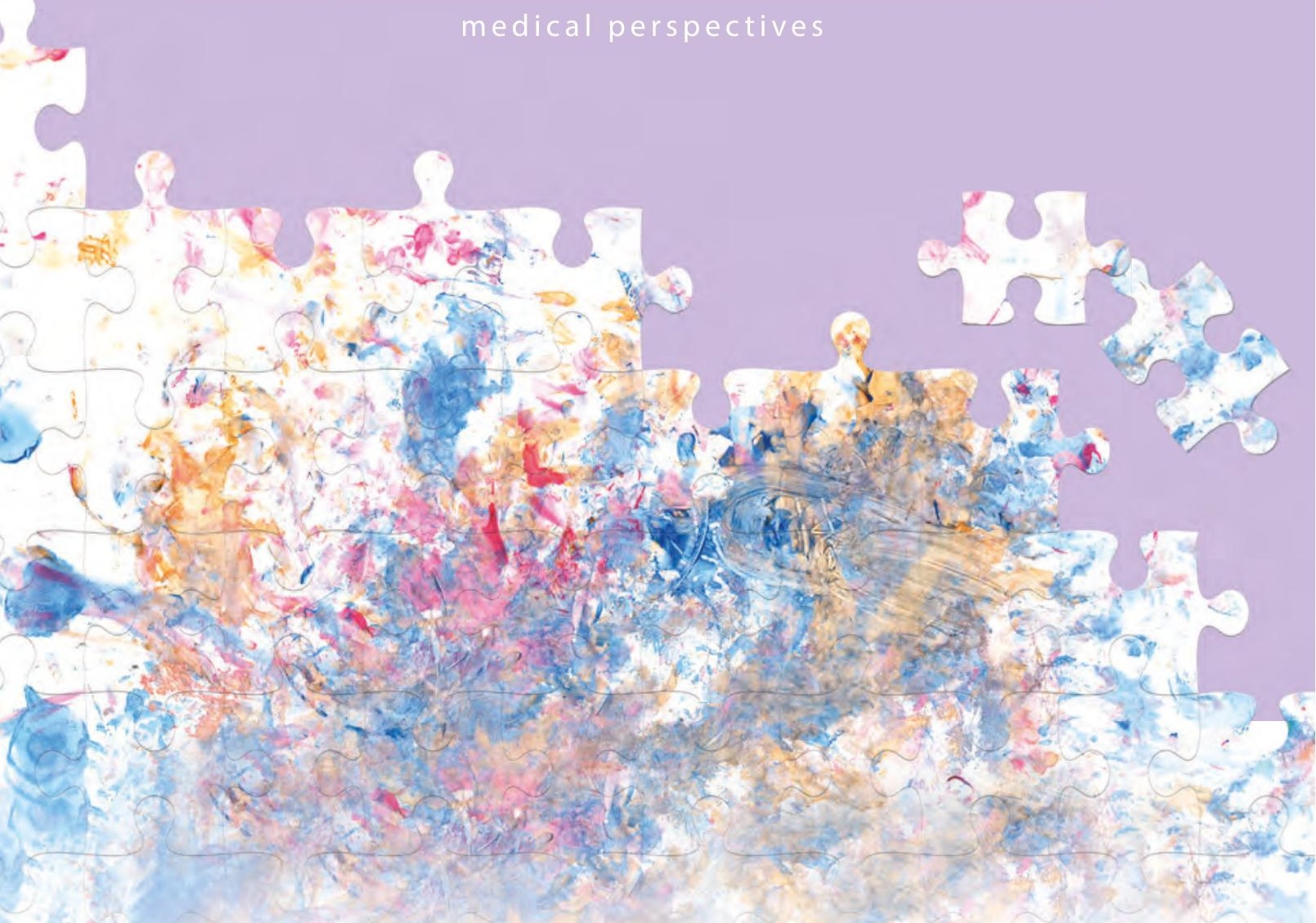

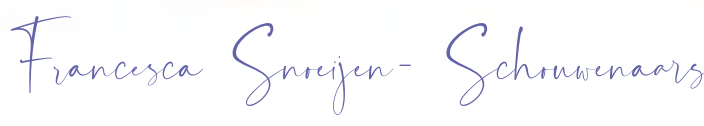



Diagnostic, neuropsychiatric and

therapeutic considerations in epilepsy

and intellectual disability

- medical perspectives - 
๑) Copyright Francesca Snoeijen-Schouwenaars, Maastricht 2019

Cover design: Rochelle van Dinter \& Jenthe Snoeijen

Layout: Tiny Wouters

Printed by: Ridderprint BV

ISBN: 978-94-6375-453-8

Printing of this thesis was financially supported by: Kempenhaeghe, the School for Mental Health and Neuroscience (MHeNS) of Maastricht University, UCB Pharma, EISAI and Ds. Visschersfonds. 


\section{Diagnostic, neuropsychiatric and therapeutic considerations in epilepsy and intellectual disability \\ - medical perspectives -}

\section{PROEFSCHRIFT}

Ter verkrijging van de graad van doctor aan de Universiteit Maastricht, op gezag van de Rector Magnificus, Prof.dr. Rianne M. Letschert volgens het besluit van het College van Decanen,

in het openbaar te verdedigen

op

woensdag 9 oktober 2019 om 12.00 uur

door

Francesca Snoeijen-Schouwenaars 


\section{Promotor}

Prof.dr. A.P. Aldenkamp

\section{Copromotores}

Dr. H.J. Schelhaas

Dr. J.G.M. Hendriksen

\section{Beoordelingscommissie}

Prof.dr. R.J. van Oostenbrugge (voorzitter)

Prof.dr. F.J.M. Feron

Prof.dr. L. Lagae (Universitair ziekenhuis Leuven, België)

Dr. D.A.M. Maes-Festen (Erasmus Medisch Centrum, Rotterdam)

Dr. M.C. Marcelis 


\section{Content}

$\begin{array}{lll}\text { Chapter } 1 & \text { General introduction } & 7\end{array}$

1.1 Definitions 9

1.2 A systematic review of neuropsychiatric comorbidities in 11 patients with both epilepsy and intellectual disability.

1.3 Aim and outline 39

Part I Pieces of the puzzle: diagnostic and neuropsychiatric 43 considerations

Chapter 2 Diagnostic exome sequencing in 100 consecutive patients 45 with both epilepsy and intellectual disability.

Chapter 3 Mood, anxiety, and perceived quality of life in adults with epilepsy and intellectual disability

Chapter $4 \quad$ Psychiatric evaluation of patients with epilepsy and intellectual 101 disability: challenges and outcomes

Part 2 Therapeutic considerations in epilepsy and intellectual disability

Chapter 5 Anti-epileptic drug treatment in patients with both epilepsy and Intellectual disability: a tale of two services

Chapter $6 \quad$ Evaluation of Perampanel in patients with ID and Epilepsy

Chapter 7 Carbamazepine and oxcarbazepine in adult patients with

Dravet syndrome: Friend or foe?

Chapter 8 General discussion

Summary

Nederlandse samenvatting

Valorisation

Dankwoord

Curriculum vitae

List of publications 



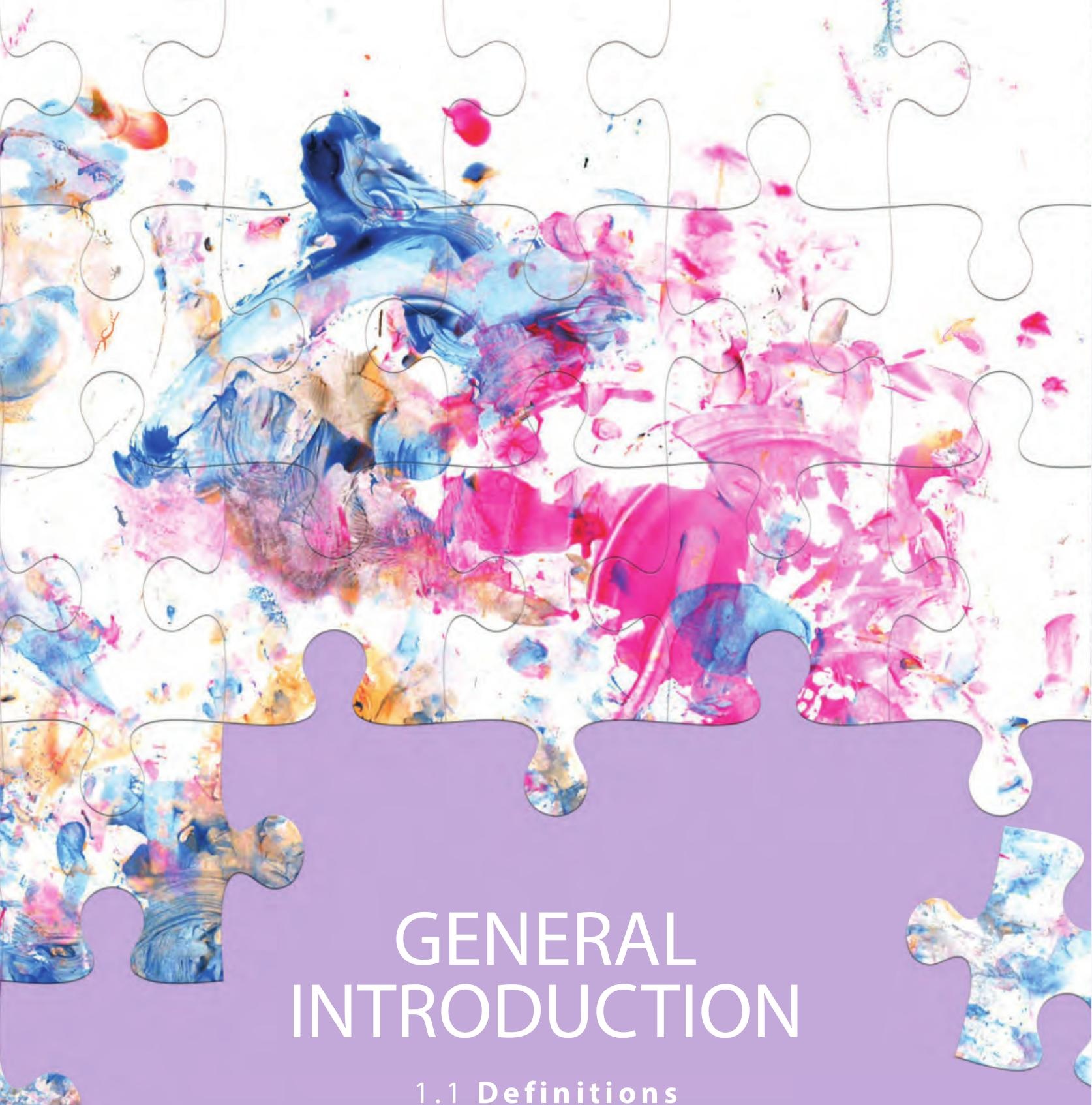

1.2 Systematic review of neuropsychiatric comorbidities in patients with both epilepsy and intellectual disability. Van Ool, Snoeijen-Schouwenaars et al. (2016).

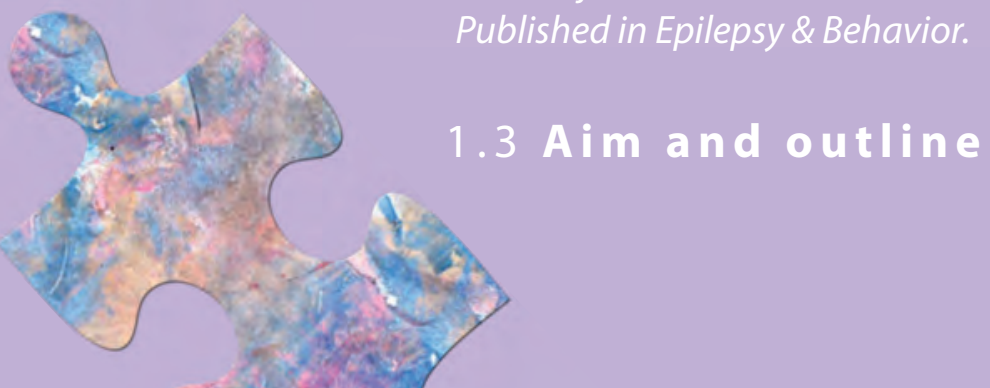




$$
\text { Chapter 1.1 }
$$

General introduction - definitions 


\section{General introduction - definitions}

\section{Epilepsy and intellectual disability}

Epilepsy is a chronic disorder of the brain characterized by a predisposition to seizure activity, and is associated with long term neurobiological, psychosocial and cognitive effects. A seizure can be defined as a transient occurrence of neurological symptoms associated with abnormal neuronal activity in the brain. ${ }^{1}$ The semiology of the seizure event will vary dependent upon the origin of the abnormal or excessive neuronal activity. The diagnosis of epilepsy has previously been defined by the International League against Epilepsy (ILAE) as at least two unprovoked seizures, at least a day apart.1 The revised practical (clinical) definition implies that epilepsy also can be considered to be present after one unprovoked seizure in individuals who have other factors that are associated with a high likelihood of a persistently lowered seizure threshold and therefore a high recurrence risk. ${ }^{2}$

Intellectual disability (ID) is defined in the Diagnostic and Statistical Manual of Mental Disorders-5 (DSM-5) as having both reduced intellectual functioning and impaired adaptive abilities to cope with the daily demands of the social environment, and should manifest during the developmental period of an individual. The impaired adaptive abilities refer to three general domains: conceptual, social and practical domain. $^{3}$

The pooled prevalence of epilepsy among patients with intellectual disability (ID) is estimated at around $22.2 \%(95 \% \mathrm{Cl} 19.6-25.0)$, the prevalence rate increasing with severity of ID. ${ }^{4}$ This is substantially higher than the reported prevalence of $1 \%$ in the general European population. ${ }^{5}$

Despite the clear relationship between epilepsy and ID, understanding this association can be complex. There is a wide range of potential pathological processes, often associated with genetic abnormalities and/or underlying structural pathology ${ }^{6}$, that might influence the neurodevelopment at varying stages. ${ }^{7,8}$ In addition, the influence of treatment resistant seizures and the effects of antiepileptic/mood stabilizing drugs complicates the unraveling of the encountered multiple comorbid conditions.

For example, studies have shown that uncontrolled epilepsy by itself can have pervasive and negative consequences on both mortality and quality of life. ${ }^{9}$

In addition to the complexity of a multifactorial etiology, the interpretation of existing literature is hampered by inconsistent definitions of ID or epilepsy and the use of small or highly selected samples. Often, literature lacks information on the extent of detail within assessments, instruments or diagnostic criteria used. 


\section{Triangle of epilepsy, ID and neuropsychiatric comorbidity}

For the full spectrum of challenging behavior, affective symptoms and psychiatric disorders, we used the term "neuropsychiatric comorbidities" in this thesis.

The assessment of behavior and neuropsychiatric comorbidity in people with epilepsy and ID can be complex and requires a multidisciplinary approach. The lack of information regarding neuropsychiatric comorbidity in patients with ID is striking, as it is known that psychiatric symptoms influence the quality of life and daily functioning of a population already known for their complex needs. ${ }^{10}$ In addition, the presence of mental and physical disorders ${ }^{11,12}$ may affect anti-epileptic drug (AED) treatment choices.

In order to create an overview of existing literature in this field, we published a systematic review focusing on neuropsychiatric comorbidities in patients who have both epilepsy and ID. 


\section{Chapter}

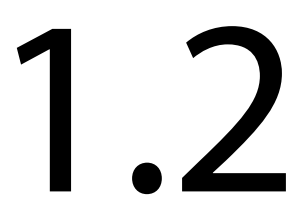

General introduction - systematic review

A systematic review of neuropsychiatric comorbidities in patients with both epilepsy and intellectual disability Van Ool, Snoeijen-Schouwenaars et al. (2016). Published in Epilepsy \& Behavior. 


\section{Abstract}

\section{Background}

Epilepsy is a neurological condition that is particularly common in people with intellectual disability (ID). The care for people with both epilepsy and ID is often complicated by the presence of neuropsychiatric disorders, defined as psychiatric symptoms, psychiatric disorders, and behavioral problems. The aim of this study was to investigate associations between epilepsy or epilepsy-related factors and neuropsychiatric comorbidities in ID patients and between ID and neuropsychiatric comorbidities in epilepsy patients.

\section{Method}

We performed a systematic review of the literature, published between January 1995 and January 2015, retrieved from PubMed/Medline, PsychInfo, and ERIC, and assessed the risk of bias using the SIGN-50 methodology. Forty-two studies were identified of which fifteen were assessed as having a low or acceptable risk of bias evaluation. Neuropsychiatric comorbidities were examined in relation to epilepsy in nine studies, in relation to epilepsy-related factors, such as seizure activity, seizure type, and medication in four studies, and in relation to the presence and degree of ID in five studies.

\section{Results and conclusion}

We can conclude that the presence of epilepsy only was not a clear determinant of neuropsychiatric comorbidity in patients with ID, although a tendency towards negative mood symptoms was identified. Epilepsy-related factors indicating a more severe form of epilepsy were associated with neuropsychiatric comorbidity as was the presence of ID as compared to those without ID in patients with epilepsy, although this should be validated in future research. A large proportion of the studies in this area are associated with a substantial risk of bias. There is a need for high-quality studies using standardized methods to enable clear conclusions to be drawn that might assist in improving the quality of care for this population 


\section{Introduction}

Epilepsy is a neurological condition that is particularly common in people with intellectual disability (ID). For the ID population, the epilepsy prevalence rates range from 20 to $30 \%$, increasing with severity of $I D,{ }^{1}$ whereas rates of around $1 \%$ are reported for the general European population. ${ }^{2}$ For the epilepsy population, $16 \%$ of individuals with epilepsy also have some degree of ID (e.g. mild, moderate, severe, or profound), ${ }^{3}$ which is much higher than the prevalence of $<1 \%$ reported for the population overall. ${ }^{4}$ The population with both epilepsy and ID has a high rate of complex needs and comorbid behavioral difficulties; these have important implications for good clinical practice and treatment of these patients. ${ }^{5}$

Epilepsy can be associated with a variety of behavioral, cognitive, and psychiatric comorbidities. ${ }^{6-8}$ The nature of these comorbidities is complicated, as many factors are involved, such as etiology and factors related to epilepsy, seizures and anti-epileptic drugs (for an overview, see ${ }^{6}$ ). In children with epilepsy, the most prevalent comorbidities include attention deficit hyperactivity disorder (ADHD), mood and anxiety disorders, autism spectrum disorders, and behavioral problems. ${ }^{8}$ Adults with epilepsy are most often diagnosed with mood and anxiety disorders and social difficulties. ${ }^{7}$

Behavioral and psychiatric comorbidities are also common in the ID population. ${ }^{9-11}$ Most common are psychotic, affective and anxiety disorders, autism spectrum disorders, and behavioral problems. ${ }^{9}$ The specific group of patients with both epilepsy and ID might present even more complicated neuropsychiatric features, since both ID and epilepsy are risk factors for such conditions. Against this background, we carried out a systematic review in order to determine which neuropsychiatric comorbidities are typical for patients who have both epilepsy and ID and which factors are associated with the neuropsychiatric comorbidities in this population. A better understanding of neuropsychiatric comorbidities in patients with both epilepsy and ID is important to improve appropriate management of epilepsy, and could eventually enhance the quality of life. As far as we are aware, no review study with this goal has been performed. 


\section{Methods}

\section{Search strategy}

The present systematic review was carried out in keeping with the guidelines of the PRISMA statement. ${ }^{12}$ The electronic databases Medline/PubMed, PsychInfo, and ERIC were searched for relevant articles on neuropsychiatric outcomes in patients with epilepsy and ID. Our search was limited to articles in the English language, published between January 1995 and January 2015. The search terms and strategy were customized for each electronic database, using Medical Subject Heading (MeSH) and title/abstract words for Medline/PubMed, and Subject Headings and keywords for PsychInfo and ERIC. The search terms for neuropsychiatric outcomes were broadly selected to ensure all relevant articles were retrieved. For each database, search terms for epilepsy, ID, and neuropsychiatric outcomes were combined in the final search (Table 1.2.1).

We used the following definitions for our key terms:

Epilepsy: Studies that reported on the presence of epilepsy or seizure disorders in their study sample. There were no restrictions on epilepsy type or seizure activity.

Intellectual disability: According to ICD-10 and DSM-5, ID is defined as having both reduced intellectual functioning (IQ below 70) and impaired adaptive abilities to cope with the daily demands of the social environment, and should manifest during the developmental period of an individual. ${ }^{13,14}$ However, as this level of intellectual functioning is not always reported in articles and the terminology for ID is often inconsistent, we included the following: articles that reported IQ levels below 70, clearly impaired adaptive abilities of individuals, or any levels of ID (or a similar term) in accordance with ICD-10 or DSM-5.

Neuropsychiatric comorbidity: In order to obtain a clear and comprehensive overview of neuropsychiatric comorbidity, we included a variety of behavioral and affective problems, psychiatric symptoms and psychiatric disorders. We based our search terms on symptoms of common ICD-10 and DSM-5 diagnoses in the ID literature and aspects of psychological instruments (see Table 1.2.1). 
Table 1.2.1: Search strings for Epilepsy, ID and Neuropsychiatric Comorbidities. ${ }^{a}$

\begin{tabular}{|c|c|}
\hline \multicolumn{2}{|l|}{ Medline/PubMed } \\
\hline Epilepsy & $\begin{array}{l}\text { epilepsy[MeSH] OR epilep*[TIAB]) OR (seizures[MeSH] OR seizure[TIAB] OR } \\
\text { seizures[TIAB] }\end{array}$ \\
\hline$I D$ & $\begin{array}{l}\text { 'Intellectual Disability'[Mesh] OR (intellectual*[TIAB] AND (disabil*[TIAB] OR } \\
\text { handicap*[TIAB] OR impair*[TIAB] OR retard*[TIAB])) OR (mental*[TIAB] AND } \\
\text { (retard*[TIAB] OR disable*[TIAB] OR deficienc*[TIAB])) OR 'Mentally Disabled } \\
\text { Persons'[Mesh] }\end{array}$ \\
\hline $\begin{array}{l}\text { Neuropsychiatric } \\
\text { comorbidities }\end{array}$ & $\begin{array}{l}\text { 'mental disorders'[MeSH] OR psychiatric[TIAB] OR depress*[TIAB] OR manic[TIAB] OR } \\
\text { bipolar[TIAB] OR adhd[TIAB] OR anxi*[TIAB] OR autis*[TIAB] OR psychosis[TIAB] OR } \\
\text { psychot*[TIAB] OR schizophren*[TIAB] OR mood[TIAB] OR affective[TIAB] OR } \\
\text { emotional*[TIAB] OR phob*[TIAB] OR psychopatholog*[TIAB] OR behavior*[TIAB] OR } \\
\text { behaviour*[TIAB] OR 'self-injury'[TIAB] OR aggress*[TIAB] OR conduct[TIAB] OR } \\
\text { violence[TIAB] OR externali*[TIAB] OR internali*[TIAB] OR (Stereotyp*[TIAB] AND } \\
\text { behavior[TIAB]) }\end{array}$ \\
\hline \multicolumn{2}{|l|}{ Psychlnfo } \\
\hline Epilepsy & epilepsy[SH] OR seizures[SH] \\
\hline ID & $\begin{array}{l}\text { 'Intellectual development disorder'[SH] OR 'cognitive impairment'[SH] OR 'intellectual } \\
\text { disability' }[\mathrm{KW}]\end{array}$ \\
\hline $\begin{array}{l}\text { Neuropsychiatric } \\
\text { comorbidities }\end{array}$ & $\begin{array}{l}\text { 'mental disorders' }[\mathrm{SH}] \text { OR psychopathology[SH] OR 'psychiatric symptoms'[SH] OR } \\
\text { 'emotional disturbances' }[\mathrm{SH}] \text { OR 'emotional state'[SH] OR 'emotion regulation' }[\mathrm{SH}] \mathrm{OR} \\
\text { 'behavior disorders' }[\mathrm{SH}] \mathrm{OR} \text { 'behavior problems' }[\mathrm{SH}] \mathrm{OR} \text { 'conduct disorder' }[\mathrm{SH}] \mathrm{OR} \\
\text { 'attention deficit disorder' }[\mathrm{SH}] \text { OR 'aggressive behavior' }[\mathrm{SH}] \text { OR 'antisocial behavior' }[\mathrm{SH}] \\
\text { OR 'oppositional defiant disorder' }[\mathrm{SH}] \text { OR 'comorbidity'[SH] OR 'stereotyped } \\
\text { behavior' }[\mathrm{SH}] \mathrm{OR} \text { 'self-destructive behavior' }[\mathrm{SH}] \mathrm{OR} \text { 'coping behavior'[SH] OR } \\
\text { externalization[SH] OR internalization[SH] }\end{array}$ \\
\hline \multicolumn{2}{|r|}{ ( } \\
\hline Epilepsy & epilepsy[SH] OR seizures[SH] \\
\hline$I D$ & 'mental retardation' $[\mathrm{SH}] \mathrm{OR}$ 'le \\
\hline $\begin{array}{l}\text { Neuropsychiatric } \\
\text { comorbidities }\end{array}$ & $\begin{array}{l}\text { 'mental disorders' }[\mathrm{SH}] \text { OR 'psychopathology' }[\mathrm{SH}] \mathrm{OR} \text { 'psychiatry' }[\mathrm{SH}] \text { OR 'mental } \\
\text { health' }[\mathrm{SH}] \text { OR 'depression' }[\mathrm{SH}] \text { OR 'behavior disorder' }[\mathrm{SH}] \mathrm{OR} \text { 'behavior problems' }[\mathrm{SH}] \\
\text { OR 'attention deficit hyperactivity disorders' }[\mathrm{SH}] \mathrm{OR} \text { 'aggression' }[\mathrm{SH}] \mathrm{OR} \\
\text { 'comorbidity' }[\mathrm{SH}] \text { OR 'self destructive behavior' }[\mathrm{SH}] \text { OR 'coping'[SH] }\end{array}$ \\
\hline
\end{tabular}

$\mathrm{ID}=$ intellectual disability, MeSH = Medical Subject Heading, $\mathrm{TIAB}=$ Title and Abstract, $\mathrm{KW}=\mathrm{Keyword}, \mathrm{SH}=\mathrm{Subject}$ heading. ${ }^{a}$ Search terms for epilepsy, intellectual disability, and neuropsychiatric outcomes were combined in the final search (i.e. epilepsy AND intellectual disability AND neuropsychiatric comorbidity).

Titles and abstracts of records were screened by one author (JO). Full-text versions of relevant articles were retrieved and assessed for eligibility. In addition, references of included articles were screened for relevance; this resulted in one additional article that met the inclusion criteria. Inclusion criteria according to type of study, type of study population, and type of outcome were:

- Type of study: Empirical and observational studies written in English, published in peer-reviewed journals. We excluded case reports, psychometric studies, commentaries, and reviews. 
- Type of study population: Humans with both epilepsy and ID included in at least part of the study sample. All types of epilepsy, levels of ID, and types of residential setting were included.

- Type of outcome: Any type of behavioral difficulties or psychiatric symptoms, specifically reported for those with both epilepsy and intellectual disability.

Studies were excluded if they did not meet the inclusion criteria or if the focus was on neuropsychiatric characteristics related to a specific intervention or to the etiology, as these topics are beyond the scope of this review. Reasons for exclusion were noted. A blinded standardized double-check of $10 \%$ of included and excluded articles was carried out by a second author $(\mathrm{JH})$. They disagreed on $6.7 \%$ of the double-checked studies; this was resolved by consensus. As a result, a total of 42 articles were considered eligible for the present review.

Next, the study quality and risk of bias were analyzed according to the SIGN-50 methodology. ${ }^{15}$ SIGN has developed checklists that can be used as a tool to assess the level of evidence of a study, and that include items regarding selection bias (e.g. 'The study indicates how many of the people asked to take part did so'), attrition bias (e.g. 'Comparison is made between full participants and those lost to follow-up'), detection bias (e.g. 'Evidence from other sources is used to demonstrate that the method of outcome assessment is valid and reliable'), and confounding (e.g. 'The main potential confounders are identified and taken into account in the design and analysis'). The principles of GRADE processes were incorporated in the development of these checklists. ${ }^{16}$ According to SIGN-50, studies are allocated to one of four categories based on their level of evidence (LE), with LE1 indicating the lowest risk of bias. As there is no checklist for cross-sectional studies, we followed the procedures of De Winter, Jansen, and Evenhuis ${ }^{17}$ and Van de Wouw, Evenhuis, and Echteld ${ }^{18}$ and adapted the SIGN methodology checklist for cohort studies in order to enable assessment of cross-sectional studies. Accordingly, the level of evidence of crosssectional studies was considered to be LE2, and cross-sectional studies without statistical analyses as non-analytical studies (LE3). The LE can be further specified as either high-quality (++), well-conducted (+) or high risk of bias (-). Our assessment of LE was only performed on the questions related to our review area. Two of the authors (JO and FS) independently analyzed the included studies according to SIGN criteria. Disagreements (21\%) were resolved by consensus. After excluding the studies with a high risk of bias, a total of 15 studies remained to be included in this systematic review (the excluded studies are described in Table S1.2.7 in the Appendix). See Figure 1.2.1 for an overview of the selection procedure. 


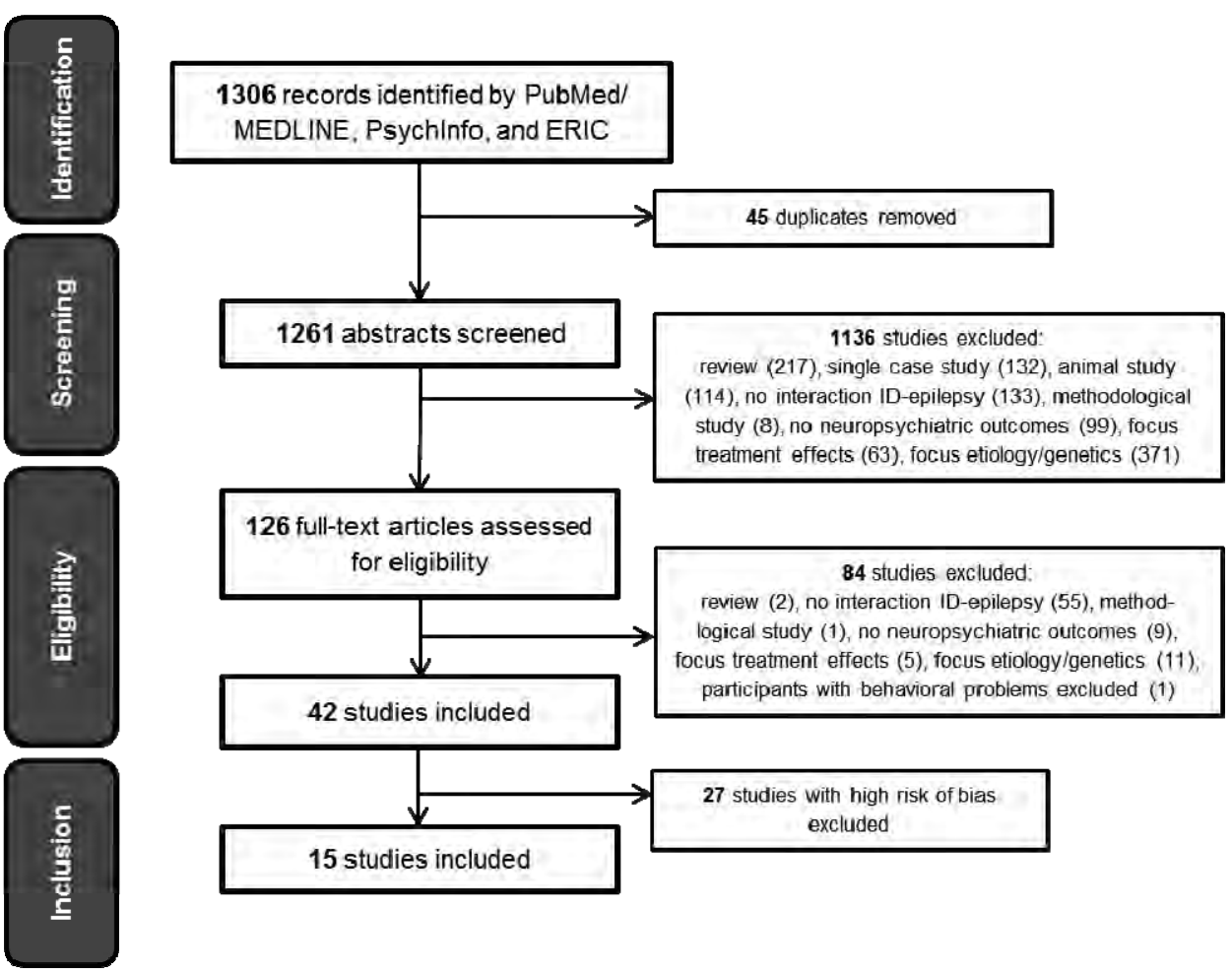

Figure 1.2.1: Summary of the study selection process.

\section{Data collection and analysis}

Study characteristics of the included articles were reviewed and the following characteristics noted, if sufficient information was available:

1. Study design, size of total sample, and number with epilepsy and ID;

2. Participants: sex, age range, and study population;

3. Level of ID and method of assessment;

4. Seizure type and method of assessment of epilepsy;

5. Neuropsychiatric outcomes and method of assessment;

6. Relevant results and conclusion. 


\section{Results}

The 15 included articles varied in their scope, and were subdivided into three categories with respect to the relation between neuropsychiatric comorbidities and: (1) the presence or absence of epilepsy in patients with ID, (2) differences in epilepsyrelated factors in patients with both epilepsy and ID, and (3) the presence or absence, or degree of ID in epilepsy patients. If an article addressed more than one category, it was allocated to both. There were nine studies in category 1 , four studies in category 2 , and four studies in category 3 . The study characteristics, study results, and level of evidence are presented by category in Tables 1.2.2-1.2.4 In order to provide a complete overview of the literature, the excluded studies with a high risk of bias are presented in Supplementary Table S1.2.7 and the methods of assessment of epilepsy, level of ID and neuropsychiatric outcomes for all studies are described in supplementary Table 1.2.6.

To avoid complexity in the text, individual statistical results are not presented here but the data are available in Tables 1.2.2-1.2.4, to which reference should be made. Only statistically significant results are presented. It should be noted that, in some cases where statistically significant associations were not established, these studies may have been underpowered, implying that statistically significant results might have been obtained if larger numbers had been included; detailed discussion of the power of each of these studies to determine statistically significant differences is not presented in this paper.

\section{Presence or absence of epilepsy}

We identified nine articles that studied the association between the presence or absence of epilepsy and neuropsychiatric comorbidities in people with ID (Table 1.2.2). Only one study was of high quality ${ }^{19}$ the rest were well-conducted. The studies consisted of participants from heterogeneous populations with respect to their age, epilepsy, level of ID, and etiology of epilepsy and ID. N varied from 90 to 3065 participants. Information about seizure type and seizure activity was often lacking.

\section{Presence of epilepsy and psychiatric symptoms and disorders}

The high quality study compared adults with ID with and without epilepsy with regard to psychiatric disorders, matched according to level of ID. ${ }^{19}$ When controlling for age, type of living environment, and sex, adults with epilepsy had more symptoms of depression and unspecified disorders (such as dementia and organic problems) than those without epilepsy. 


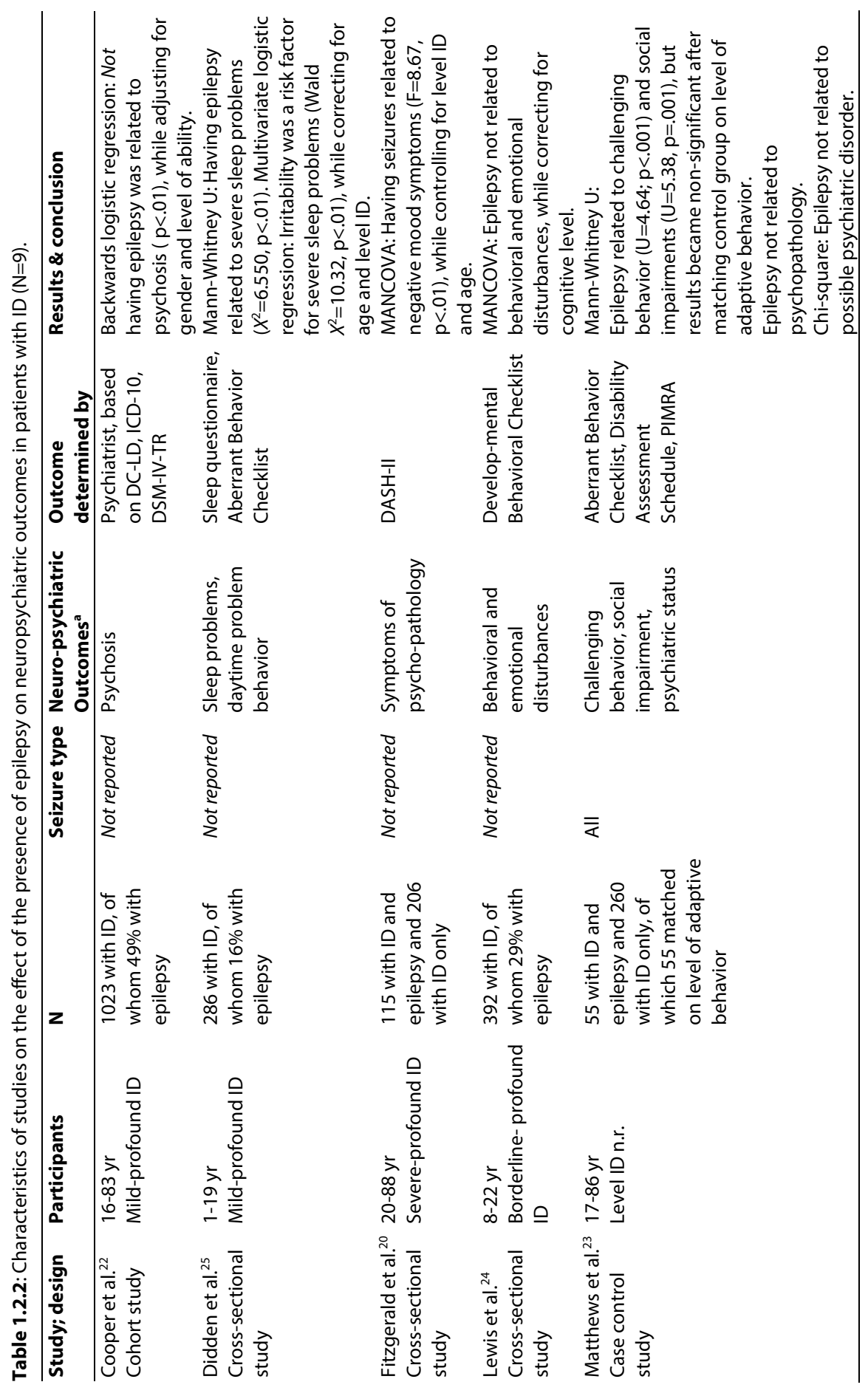




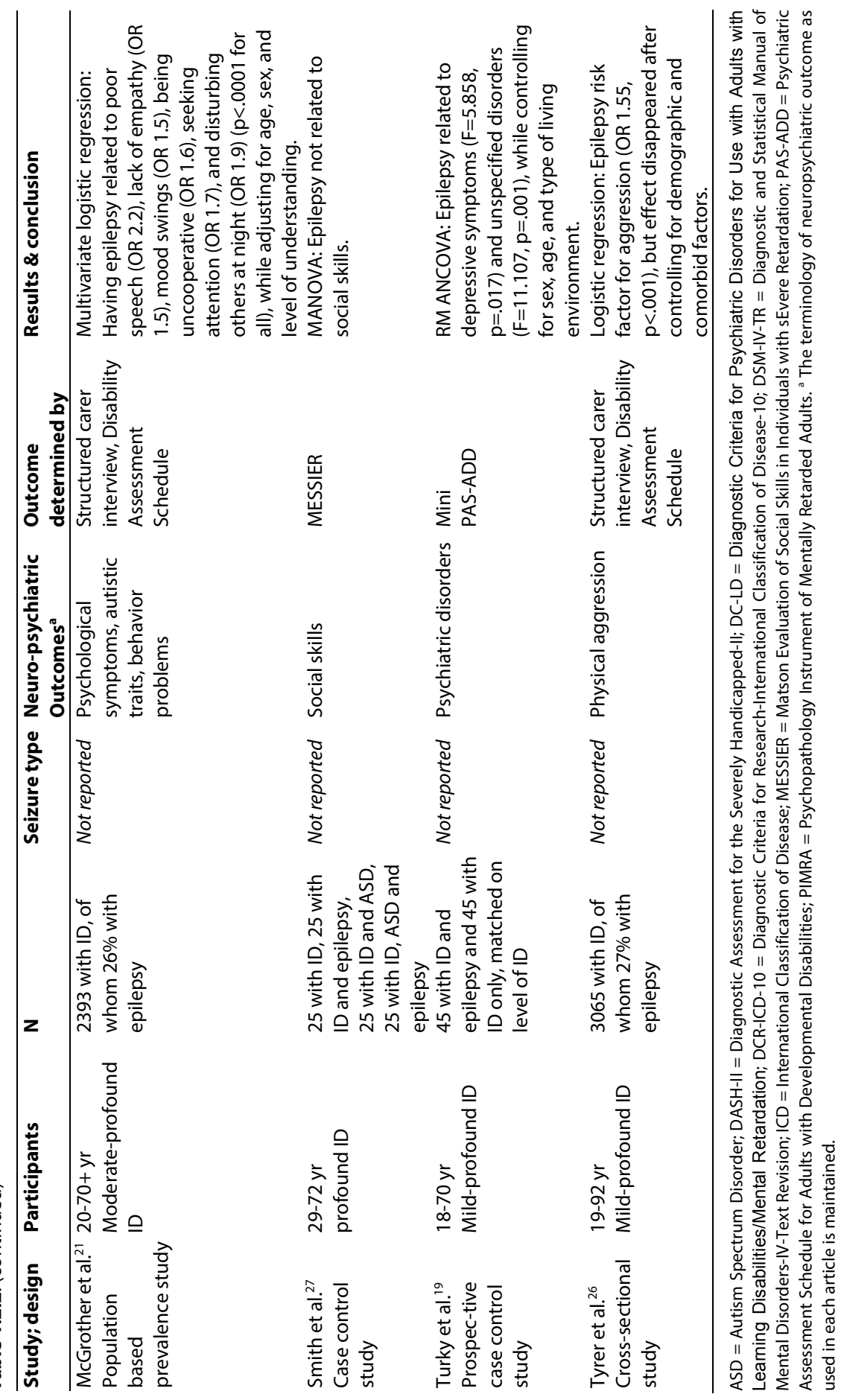


This is in line with results from Fitzgerald et al., ${ }^{20}$ who found more negative mood symptoms in adults with seizures than in those without, after controlling for level of ID and age. In addition, McGrother et al. ${ }^{21}$ reported that, after controlling for age, sex, and level of intellectual understanding, adults with epilepsy and ID had higher rates of lack of empathy, mood swings, and poor speech than adults without epilepsy.

In contrast, Cooper et al. ${ }^{22}$ found that adults with epilepsy were less likely to have psychosis, after adjustment for age and level of ability. There were two studies that did not report associations between the presence of epilepsy and psychiatric symptoms. More specifically, Matthews et al. ${ }^{23}$ concluded that having epilepsy was not associated with any psychopathology in adults with ID and Lewis et al. ${ }^{24}$ reported that having epilepsy was not associated with emotional disturbances in individuals aged 8 to 22 , after correcting for cognitive level.

\section{Presence of epilepsy and behavioral problems}

The presence of epilepsy was associated with higher rates of being uncooperative, seeking attention, and disturbing others at night in adults with ID, after controlling for age, sex, and level of intellectual understanding. ${ }^{21}$ Didden et al. ${ }^{25}$ found that children and adolescents with epilepsy had more severe sleep problems than those without epilepsy, which were related to more irritability. Challenging behavior and social impairments appeared to be more prevalent in adults with epilepsy and ID, compared to adults without epilepsy. ${ }^{23}$ These differences, however, became non-significant when comparisons were restricted to a subgroup of patients with ID with adaptive behavior levels similar to levels of patients with epilepsy and ID. Tyrer et al. ${ }^{26}$ find a similar trend; epilepsy was associated with aggression in adults with ID, but this effect became non-significant after controlling for demographic and comorbid factors. Having epilepsy was not related to behavioral disturbances in individuals aged 8 to $22,{ }^{24}$ nor to social skills in adults. ${ }^{27}$

\section{Epilepsy-related factors}

We identified four studies that investigated the role of epilepsy-related and other factors on neuropsychiatric outcomes in adolescents and adults with both epilepsy and ID (Table 1.2.3). 


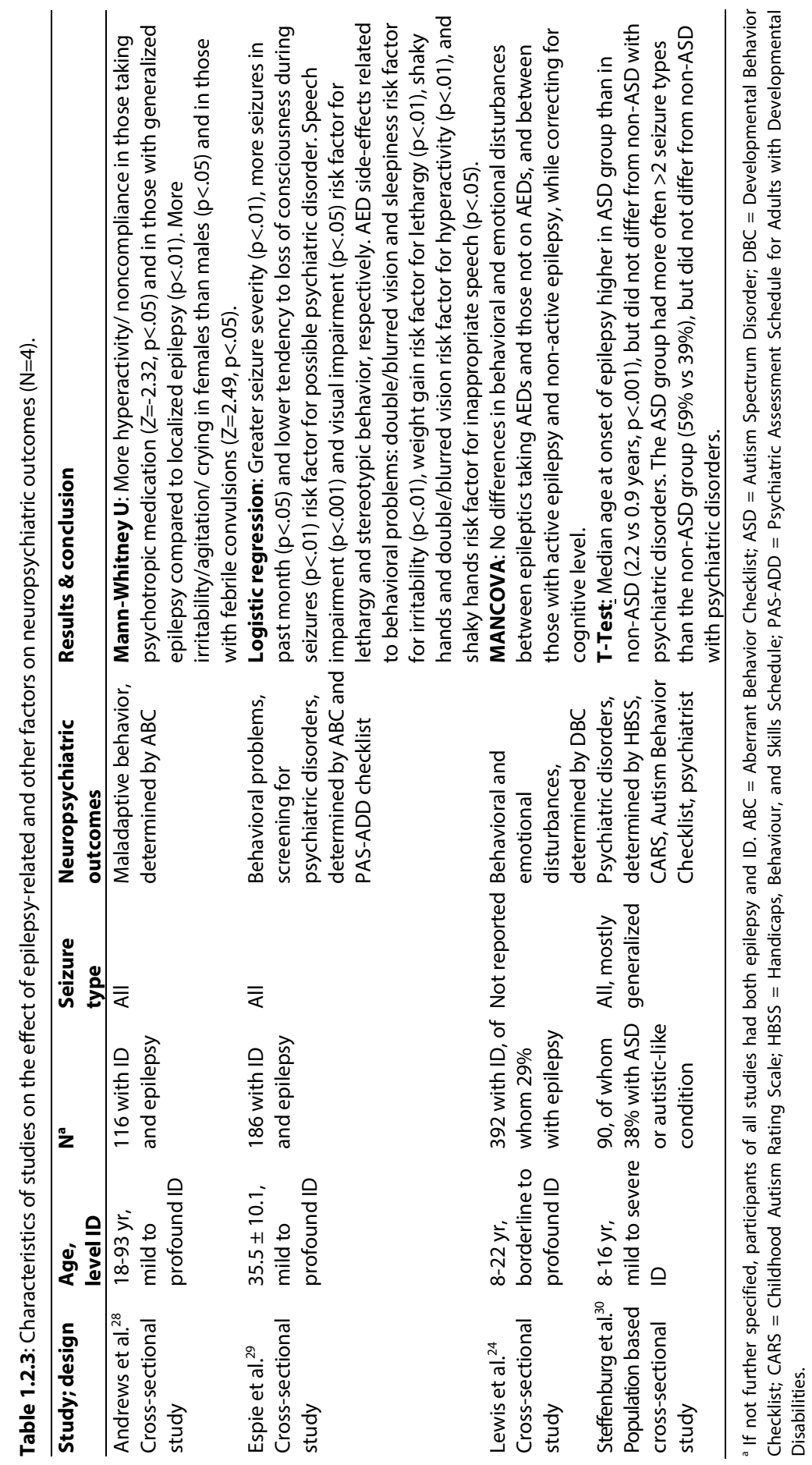




\section{Seizure type}

Three studies revealed a relationship between neuropsychiatric outcomes and seizure type, number of seizure types, or seizure severity. ${ }^{28-30}$ Steffenburg et al. ${ }^{30}$ found that children and adolescents with autism spectrum disorder and ID were more likely to have more than two seizure types than ID patients without autism spectrum disorder. They did not, however, find a difference in number of seizure types between ID patients with autism spectrum disorder and ID patients with other psychiatric disorders. Andrews et al. ${ }^{28}$ found that adult and elderly patients with generalized epilepsy showed more hyperactivity and non-compliance than those with localized epilepsy. Furthermore, greater seizure severity was an independent risk factor for a possible psychiatric disorder in adults with ID. ${ }^{29}$

\section{Seizure activity}

Only two studies investigated associations between seizure activity and neuropsychiatric outcomes; the results of these two studies were not consistent. ${ }^{24,29}$ Espie et al. ${ }^{29}$ showed that having more seizures in the past month was an independent risk factor for psychiatric disorders in adults with ID. Lewis et al. ${ }^{24}$ did not, however, find a relation between active versus non-active epilepsy and behavioral or emotional disturbances in individuals aged 8-22, after adjusting for cognitive level.

\section{Other factors}

Four well-conducted studies examined associations between other factors and neuropsychiatric comorbidities. Irritability, agitation, and crying appeared to be more prevalent in women compared to men and in adults who had a history of febrile convulsions. ${ }^{28}$ Steffenburg et al. ${ }^{30}$ found that children and adolescents with autism spectrum disorder and ID had a higher age of epilepsy onset than those without autism spectrum disorder. Furthermore, a lower tendency to loss of consciousness during seizures was an independent risk factor for having a possible psychiatric disorder and having a sensory disability was positively related to behavioral problems in adults. ${ }^{29}$ More specifically, speech impairment was associated with lethargy, whereas a visual impairment was associated with stereotypical behavior. Adverse events due to antiepileptic drugs were positively associated with behavioral problems in adults. More specifically, double/blurred vision and sleepiness were risk factors for irritability, weight gain was a risk factor for lethargy, shaky hands and double or blurred vision were risk factors for hyperactivity, and shaky hands was a risk factor for inappropriate speech. ${ }^{29}$ These studies might have identified_important factors that 
interact with other variables in explaining neuropsychiatric outcomes. Their findings are not, however, validated by other studies and should therefore be interpreted carefully.

\section{Presence and level of ID}

Our search revealed five studies that examined the presence or level of ID on neuropsychiatric outcomes in epilepsy patients (Table 1.2.4). One study received a high quality evaluation; ${ }^{31}$ the results of the remaining four studies had an acceptable risk of bias. It should be noted that the study samples of three studies predominantly included participants with mild ID and that none of the studies corrected for epilepsy characteristics in the analyses.

\section{Presence of ID and neuropsychiatric outcomes}

The high quality study examined psychiatric disorders in children with epilepsy, $40 \%$ of whom were diagnosed with ID. ${ }^{31}$ They found significantly higher rates of autism spectrum disorder in children with ID compared to those without, while adjusting for epilepsy duration, seizure type, and known/unknown etiology. Having ID was not related to ADHD, depression, developmental coordination disorder, or anxiety. Matsuura et al. ${ }^{32}$ investigated psychiatric disorders in a mixed sample of children and adults with epilepsy. Of these, $23 \%$ had ID, predominantly of a mild level. They showed that the presence of ID was the strongest predictor for having a psychiatric disorder. Hilger et al. ${ }^{33}$ studied postictal psychosis in adults with temporal lobe epilepsy of whom $5 \%$ had impaired intellectual functioning. Postictal psychosis was associated with impaired intellectual functioning and ictal fear.

Level of ID

Two studies examined associations between level of ID and behavioral problems.28,29 Andrews et al. $^{28}$ found that adults with a moderate level of ID showed more hyperactivity/non-compliance and used more inappropriate speech than adults with a mild ID. Espie et al. ${ }^{29}$ found that a more severe level of ID was a risk factor for lethargy and stereotypic behavior in adults. Thus, both studies concluded that patients with more severe ID have more behavioral problems, although the specific type of problematic behavior differed. To our knowledge, these are the only studies that examined the relation between level of ID and neuropsychiatric outcomes in epilepsy patients. 


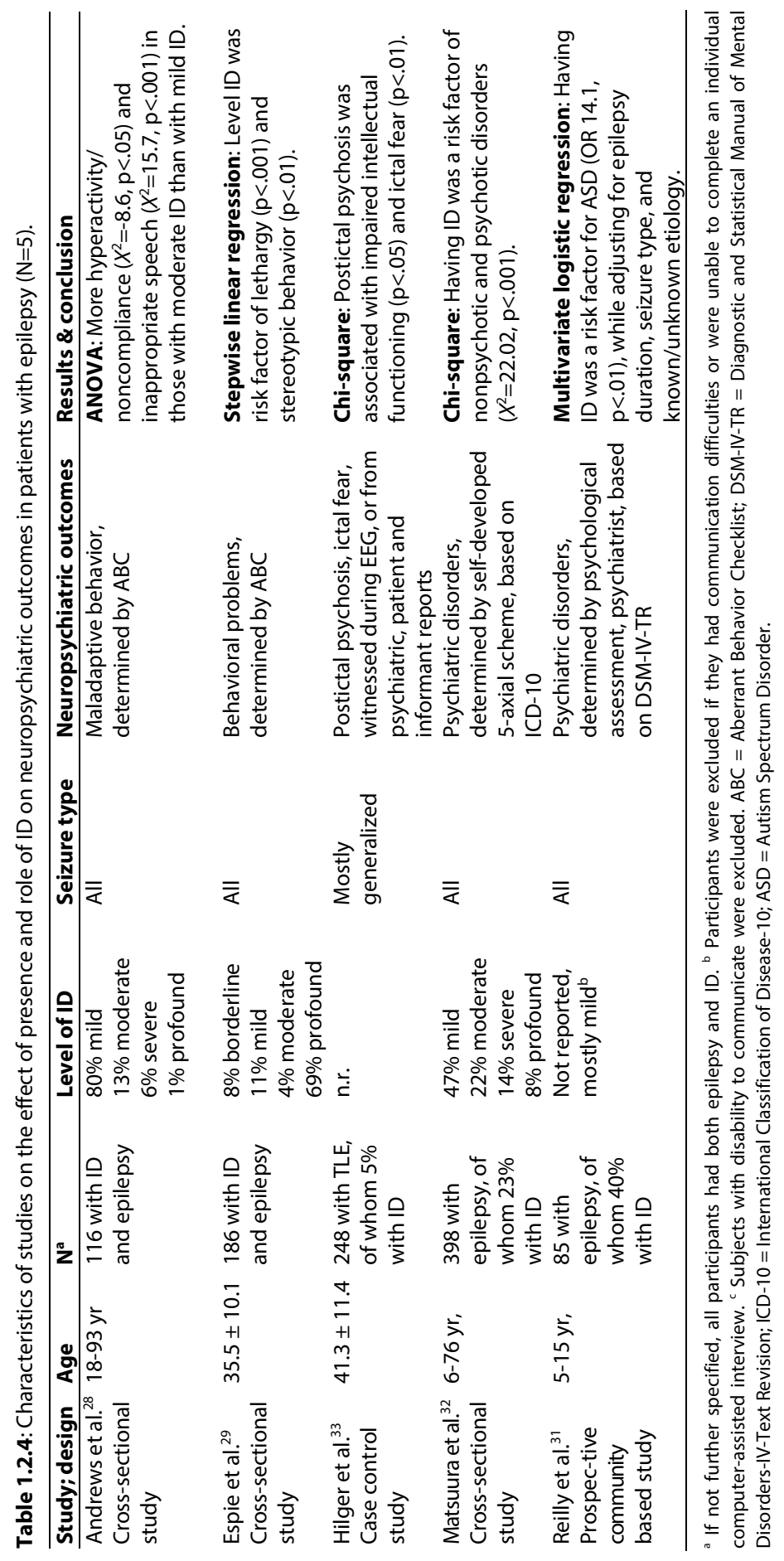




\section{Discussion}

This systematic review aimed to clarify whether there are significant associations between epilepsy or epilepsy-related factors and neuropsychiatric comorbidities in the ID population and between ID and neuropsychiatric comorbidities in epilepsy patients. From an initial total of 1306 records, we included 15 studies with a high quality or acceptable risk of bias evaluation, ${ }^{15}$ comprising 461 children and adolescents, 7742 adults and elderly, and 392 patients of mixed age groups. An overview of results is presented in Table 1.2.5. As most studies had a cross-sectional design, it is not possible to draw firm conclusions about causality.

With respect to the relation between the presence or absence of - any type of epilepsy and neuropsychiatric outcomes in ID, there was an indication that having epilepsy was significantly related to higher rates of negative mood symptoms in adults and elderly with ID, ${ }^{19-21}$ including depressive symptoms, negative mood, and mood swings. Having epilepsy was, however, not associated with emotional disturbances. ${ }^{24}$ The association between epilepsy and mood symptoms would be in line with results from a systematic review and meta-analysis on active and lifetime depression rates in epilepsy patients (including patients without ID), demonstrating increased odds in epilepsy patients compared to those without. ${ }^{34} \mathrm{As}$ far as behavioral problems are concerned, the results were inconsistent. Most studies did not, however, demonstrate a significant association between the presence of epilepsy and behavioral problems. ${ }^{23,24,26,27}$

The role of epilepsy-related and other factors on neuropsychiatric comorbidities in ID patients were examined in only five studies. Generally, the results indicate that more severe epilepsies, including generalized seizures, greater seizure severity, higher seizure frequency, and higher number of seizure types, were risk factors for behavioral problems and psychiatric disorders. ${ }^{29,30,33}$ These epilepsy factors can generate a negative impact on cognitive processes and brain development, ${ }^{6,7}$ and might, therefore, result in the development of neuropsychiatric problems.

Finally, four studies looked into the relation between the presence and degree of ID and neuropsychiatric outcomes in epilepsy patients and showed a significant association. More specifically, having ID was related to higher rates of autism spectrum disorder, postictal psychosis, and both psychotic and non-psychotic disorder. $^{31-33}$ The degree of the ID seemed relevant with respect to behavioral problems, a more severe ID being significantly associated with more behavioral problems in adults with epilepsy. ${ }^{28,29}$ These tentative results on the associations between ID and neuropsychiatric comorbidities in epilepsy patients should, however, be validated by future research. 
Table 1.2.5: Summary of findings on neuropsychiatric outcomes in patients with ID and epilepsy.

\begin{tabular}{|c|c|}
\hline \multicolumn{2}{|l|}{ Presence of epilepsy } \\
\hline Psychiatric characteristics & Behavioral problems \\
\hline+ Negative mood ${ }^{20}$ & + Poor speech, being uncooperative, disturbing \\
\hline + Depressive symptoms and unspecified disorders ${ }^{19}$ & others at night ${ }^{21}$ \\
\hline $\begin{array}{l}\text { + Lack of empathy, mood swings }{ }^{21} \\
\text { - Less psychosis }\end{array}$ & $\begin{array}{l}\text { + Severe sleep problems, which were related to } \\
\text { irritability }^{25}\end{array}$ \\
\hline $\begin{array}{l}\text { No association with emotional disturbances }{ }^{24} \text { and } \\
\text { psychopathology }^{23}\end{array}$ & $\begin{array}{l}\text { No association with behavioral disturbances, }{ }^{24} \\
\text { behavioral problems and social impairment, }{ }^{23} \text { social } \\
\text { skills }^{27} \text { and physical aggression }\end{array}$ \\
\hline \multicolumn{2}{|l|}{ Epilepsy-related factors } \\
\hline Seizure type & Other \\
\hline + Generalized epilepsy: hyperactivity, & + Higher age at onset: ASD $\left[{ }^{30}\right]$ \\
\hline non-compliance ${ }^{28}$ & + Febrile convulsions, females: irritability, agitation, \\
\hline + More than 2 seizure types: ASD $^{30}$ & crying $^{28}$ \\
\hline + Seizure severity: possible psychiatric disorder ${ }^{29}$ & $\begin{array}{l}\text { - Loss of consciousness: fewer psychiatric } \\
\text { disorders }\end{array}$ \\
\hline Seizure frequency & + Sensory disabilities: behavioral problems ${ }^{29}$ \\
\hline $\begin{array}{l}+ \text { Higher seizure frequency: possible psychiatric } \\
\text { disorder }\end{array}$ & + AED adverse events: behavioral problems ${ }^{29}$ \\
\hline \multicolumn{2}{|l|}{$\begin{array}{l}\text { No association between active epilepsy and } \\
\text { behavioral and emotional disturbances }{ }^{24}\end{array}$} \\
\hline \multicolumn{2}{|l|}{ ID } \\
\hline Presence of ID & Level of ID \\
\hline + Non-psychotic and psychotic disorders ${ }^{32}$ & + Moderate ID (vs. mild ID): hyperactivity, non- \\
\hline + Autism Spectrum Disorder ${ }^{31}$ & compliance and inappropriate speech ${ }^{28}$ \\
\hline+ Postictal psychosis ${ }^{33}$ & + More severe ID: lethargy, stereotypic behavior ${ }^{29}$ \\
\hline
\end{tabular}

The main limitation of this review is that many of the included studies examined highly heterogeneous patient groups, with different types of epilepsies and seizures, multiple levels of ID combined, a wide age range, and a lack of information regarding the etiology of epilepsy and ID. In addition, the terminology used to describe similar conditions was not consistent, such as behavioral problems, maladaptive behavior, behavioral disturbances, and challenging behavior, even in studies in which the same instrument was used. This makes it very difficult to compare and generalize results from multiple studies on similar conditions in order to draw firm conclusions. Also, in the designs of several studies, potential confounding variables, such as the effects of antiepileptic drugs and known etiology of ID and epilepsy, were not included. Finally, the wide variety of instruments to assess psychiatric disorders or symptoms, often with poor psychometric properties, are of concern as they might weaken or bias the results. We highly recommend the use of validated instruments or assessment by trained professionals, based on the most recent editions of ICD or DSM and following structured guidelines developed primarily for the ID population. ${ }^{35}$ 
This review illustrates the wide range of neuropsychiatric problems among patients with ID and epilepsy, but also identifies major gaps and limitations in the literature. As most studies in the present review had a cross-sectional design, several questions remained unanswered, and future studies should study patients with both epilepsy and ID longitudinally, ideally along with a matching control group of patients without ID and a comparison group without epilepsy. A focus on epilepsyrelated factors is recommended. Also, studying more homogenous groups of patients, both with regard to epilepsy syndrome and degree of ID, might help to clarify remaining issues. More attention should be paid to potential confounding variables. We encourage researchers to use validated and internationally-used methods in order to promote high quality research and generalized conclusions, and to try to use the same terminology. 


\section{References}

1. Bowley C, Kerr M. Epilepsy and intellectual disability. J Intellect Disabil Res 2000;44:529-43.

2. Forsgren $L$, Beghi $E$, Ôun A, Sillanpää $M$. The epidemiology of epilepsy in Europe - a systematic review. Eur J Neurol 2005;12:245-53.

3. Morgan $\mathrm{CL}$, Baxter H, Kerr MP. Prevalence of epilepsy and associated health service utilization and mortality among patients with intellectual disability. Am J Ment Retard 2003;108:293-300.

4. Westerinen $H$, Kaski M, Virta LJ, Almqvist F, livanainen M. Age-specific prevalence of intellectual disability in Finland at the beginning of new millennium - multiple register method. J Intellect Disabil Res 2014;58:285-95.

5. Kerr M, Gil-Nagel A, Glynn M, Mula M, Thompson R, Zuberi SM. Treatment of behavioral problems in intellectually disabled adult patients with epilepsy. Epilepsia 2013;54(Suppl. 1):34-40.

6. Aldenkamp AP, Bodde N. Behaviour, cognition and epilepsy. Acta Neurol Scand 2005;112(Suppl. 182):19-25.

7. Lin JJ, Mula M, Hermann BP. Uncovering the neurobehavioural comorbidities of epilepsy over the lifespan. Lancet 2012;380:1180-92.

8. Hamiwka L, Jones JE, Salpekar J, Caplan R. Child psychiatry. Epilepsy Behav 2011;22:38-46.

9, Cooper SA, Smiley E, Morrison J, Williamson A, Allan L. Mental ill-health in adults with intellectual disabilities: prevalence and associated factors. Br J Psychiatry 2007;198:27-35.

10. Cowley A, Holt G, Bouras N, Sturmey P, Newton JT, Costello H. Descriptive psychopathology in people with mental retardation. J Nerv Ment Dis 2004;192:232-7.

11. Koskentausta T, Livanainen M, Almqvist F. Psychiatric disorders in children with intellectual disability. Nord J Psychiatry 2002;56:126-31.

12. Liberati A, Altman DG, Tetzlaff J, Mulrow C, Gøtzsche PC, loannidis JPA, et al. The PRISMA statement for reporting systematic reviews and meta-analyses of studies that evaluate health care interventions: explanation and elaboration. PLoS Med 2009;6, e1000100.

13. American Psychiatric Association. Diagnostic and statistical manual of mental disorders. 5th ed. Arlington, VA: American Psychiatric Publishing; 2013.

14. World Health Organization. The ICD-10 classification of mental and behavioural disorders: clinical descriptions and diagnostic guidelines. Geneva: World Health Organization; 1992.

15. Scottish Intercollegiate Guidelines Network. SIGN 50. A guideline developer's handbook. Edinburgh: SIGN Executive; 2008.

16. Guyatt GH, Oxman AD, Vist GE, Kunz R, Falck-Ytter Y, Alonso-Coello $P$, et al. GRADE: an emerging consensus on rating quality of evidence and strength of recommendations. BMJ 2008;336:924-6.

17. De Winter CF, Jansen AAC, Evenhuis HM. Physical conditions and challenging behaviour in people with intellectual disability: a systematic review. J Intellect Disabil Res 2011;55:675-98.

18. Van deWouw E, Evenhuis HM, Echteld MA. Prevalence, associated factors and treatment of sleep problems in adults with intellectual disability: a systematic review. Res Dev Disabil 2012;33:1310-32.

19. Turky A, Felce D, Jones G, Kerr M. A prospective case control study of psychiatric disorders in adultswith epilepsy and intellectual disability. Epilepsia 2011;52:1223-30.

20. FitzgeraldME,Matson JL, Barker A. Symptoms of psychopathology in adults with intellectual disability and seizures. Res Dev Disabil 2011;32:2263-6.

21. McGrother CW, Bhaumik S, Thorp CF, Hauck A, Branford B, Watson JM. Epilepsy in adults with intellectual disabilities: prevalence, associations and service implications. Seizure 2006;15:376-86.

22. Cooper SA, Smiley E, Morrison J, Allan L, Williamson A, Finlayson J, et al. Psychosis and adults with intellectual disabilities. Prevalence, incidence, and related factors. Soc Psychiatry Psychiatr Epidemiol 2007:42:530-6.

23. Matthews T, Weston N, Baxter H, Felce D, Kerr M. A general practice-based prevalence study of epilepsy among adults with intellectual disabilities and of its association with psychiatric disorder, behaviour disturbance and carer stress. J Intellect Disabil Res 2008;52:163-73.

24. Lewis JN, Tonge BJ, Mowat DR, Einfeld SL, Siddons HM, Rees VW. Epilepsy and associated psychopathology in young people with intellectual disability. J Paediatr Child Health 2000;36:172-5. 
25. Didden R, Korzilius H, Van Aperlo B, Van Overloop C, De Vries M. Sleep problems and daytime problem behaviours in children with intellectual disability. J Intellect Disabil Res 2002;46:537-47.

26. Tyrer F, McGrother CW, Thorp CF, Donaldson M, Bhaumik S, Watson JM, et al. Physical aggression towards others in adults with learning disabilities: prevalence and associated factors. J Intellect Disabil Res 2006;50:295-304.

27. Smith KRM, Matson JL. Social skills: differences among adults with intellectual disabilities, co-morbid autism spectrum disorders and epilepsy. Res Dev Disabil 2010;31:1366-72.

28. Andrews TM, Everitt AD, Sander JWAS. A descriptive survey of long-term residents with epilepsy and intellectual disability at the Chalfont Centre: is there a relationship betweenmaladaptive behaviour andmagnetic resonance imaging findings? J Intellect Disabil Res 1999;43:475-83.

29. Espie CA, Watkins J, Curtice L, Espia A, Duncan R, Ryan JA, et al. Psychopathology in people with epilepsy and intellectual disability: an investigation of potential explanatory variables. J Neurol Neurosurg Psychiatry 2003;74:1485-92.

30. Steffenburg S, Steffenburg U, Gillberg C. Autism spectrum disorders in children with active epilepsy and learning disability: comorbidity, pre- and perinatal background, and seizure characteristics. Dev Med Child Neurol 2003;45:724-30.

31. Reilly C, Atkinson P, Das KB, Chin RFMC, Aylett SE, Burch V, et al. Neurobehavioral comorbidities in children with active epilepsy: a population-based study. Pediatrics 2014;133:e1586-93.

32. Matsuura M, Oana $Y$, Kato $M$, Kawana A, Kan $R$, Kubota $H$, et al. A multicenter study on the prevalence of psychiatric disorders among newreferrals for epilepsy in Japan. Epilepsia 2003;44:107-14.

33. Hilger E, Zimprich F, Jung R, Pataraia E, Baumgartner C, Bonelli S. Postictal psychosis in temporal lobe epilepsy: a case-control study. Eur J Neurol 2013;20:955-61.

34. Fiest KM, Kykeman J, Patten SB,Wiebe S, Kaplan GG, Maxwell CJ, et al. Depression in epilepsy. A systematic review and meta-analysis. Neurology 2013;80:590-9.

35. Deb S, Matthews T, Holt G, Bouras N. Practice guidelines for the assessment and diagnosis of mental health problems in adults with intellectual disability. London: Pavilion Press; 2001.

36. Arshad S, Winterhalder R, Underwood L, Kelesidi K, Chaplin E, Kravariti E, et al. Epilepsy and intellectual disability: does epilepsy increase the likelihood of comorbid psychopathology? Res Dev Disabil 2011;32:353-7.

37. Buono S, Scannella F, Palmigiano MB, Elia M, Kerr M, Di Nuovo S. Self-injury in people with intellectual disability and epilepsy: a matched controlled study. Seizure 2012;21:160-4.

38. Brylewski JE,Wiggs L. A questionnaire survey of sleep and night-time behaviour in a communitybased sample of adults with intellectual disability. J Intellect Disabil Res 1998;42:154-62.

39. Chadwick O, Piroth N, Waller J, Bernard S, Taylor E. Factors affecting the risk of behaviour problems in children with severe intellectual disability. J Intellect Disabil Res 2000;44:108-23.

40. Chadwick O, Kusel Y, Cuddy M. Factors associated with the risk of behaviour problems in adolescents with severe intellectual disabilities. J Intellect Disabil Res 2008;52:864-76.

41. Chung MC, Cassidy G. A preliminary report on the relationship between challenging behaviour and epilepsy in learning disability. Eur J Psychiatry 2001;15:23-32.

42. Deb S. Mental disorder in adults with mental retardation and epilepsy. Compr Psychiatry 1997;38:17984.

43. Deb S. Electrophysiological correlates of psychopathology in individualswithmental retardation and epilepsy. J Intellect Disabil Res 1995;39:129-35.

44. Deb S, Joyce J. Psychiatric illness and behavioural problems in adults with learning disability and epilepsy. Behav Neurol 1998;11:125-9.

45. Deb S, Thomas M, Bright C. Mental disorder in adults with intellectual disability. 2: The rate of behaviour disorders among a community-based population aged between 16 and 64 years. J Intellect Disabil Res 2001;45:506-14.

46. Johnson CR, Lowengrub JA, Lubetsky MJ. Psychiatric and behavior disorders in childrenwithmental retardation and seizure disorder. J Dev Phys Disabil 1995;7:243-52.

47. Jones S, Cull CA. An investigation of behaviour disturbance and adaptive behaviour of children with severe intellectual disabilities and epilepsy: a comparative study. J Appl Res Intellect Disabil 1998;11:247-54. 
48. Lacey CJ, Salzberg MR, D'Souza WJ. Risk factors for psychological distress in community-treated epilepsy. Epilepsy Behav 2014;35:1-5.

49. Matson JL, Bamburg JW, Mayville EA, Khan I. Seizure disorders in people with intellectual disability: an analysis of differences in social functioning, adaptive functioning and maladaptive behaviours. J Intellect Disabil Res 1999;43:531-9.

50. Matsuura M, Adachi N, Muramatsu R, Kato M, Onuma T, Okubo Y, et al. Intellectual disability and psychotic disorders of adult epilepsy. Epilepsia 2005;46(Suppl. 1):11-4.

51. Molteno G, Molteno CD, Finchilescu G, Dawes ARL. Behavioural and emotional problems in children with intellectual disability attending special schools in Cape Town, South Africa. J Intellect Disabil Res 2001;45:515-20.

52. Pawar DG, Akuffo EO. Comparative survey of comorbidities in people with learning disability with and without epilepsy. Psychiatr Bull 2008;32:224-6.

53. Piazzini A, Bravi F, Edefonti V, Turner K, Vignoli A, Ferraroni M, et al. Aggressive behavior and epilepsy: a multicenter study. Epilepsia 2012;53:e174-9.

54. Ring H, Zia A, Lineman S, Himlok K. Interactions between seizure frequency, psychopathology, and severity of intellectual disability in a population with epilepsy and a learning disability. Epilepsy Behav 2007;11:92-7.

55. Roberts C, Yoder PJ, Kennedy CH. Descriptive analysis of epileptic seizures and problembehavior in adults with developmental disabilities. Am J Ment Retard 2005;110:405-12.

56. Smith KRM, Matson JL. Behavior problems: differences among intellectually disabled adults with comorbid autism spectrum disorders and epilepsy. Res Dev Disabil 2010;31:1062-9.

57. Smith KRM, Matson JL. Psychopathology: differences among adults with intellectually disabled, comorbid autism spectrum disorders and epilepsy. Res Dev Disabil 2010;31:743-9.

58. Steffenburg S, Gillberg C, Steffenburg U. Psychiatric disorders in children and adolescents with mental retardation and active epilepsy. Arch Neurol 1996;53:904-12.

59. Turkistani IYA. Epilepsy in learning disabilities: relevance and association withmental illness and behavioural disturbances. J Learn Disabil 2004;8:89-99. 


\section{Appendix 1.2}

Supplementary Table 1.2.6: Assessment methods of epilepsy and ID of all studies.

\begin{tabular}{|c|c|c|}
\hline \multirow[t]{2}{*}{ Study } & \multicolumn{2}{|l|}{ Measure } \\
\hline & Epilepsy & Level ID \\
\hline Andrews et al. ${ }^{28}$ & EEG and CT data & IQ tests, clinical notes \\
\hline Arshad et al. ${ }^{36}$ & N.r. & Psychiatrists specialized in people with ID \\
\hline Brylewski et al. ${ }^{37}$ & Parent/patient interview & N.r. \\
\hline Buono et al. ${ }^{38}$ & N.r. & N.r. \\
\hline Chadwick et al. ${ }^{40}$ & Parent/carer reports & Reported by special need schools \\
\hline Chadwick et al..$^{39}$ & Parental interview & Reported by special need schools \\
\hline Chung et al. ${ }^{41}$ & Medical records & Rated by keyworkers \\
\hline Cooper et al. ${ }^{9}$ & $21^{\text {st }}$ Health Check & $\begin{array}{l}\text { Vineland Adaptive Behavior Scale, results previous IQ } \\
\text { tests }\end{array}$ \\
\hline Cooper et al. ${ }^{22}$ & N.r. & Reported by family physician or general practitioner \\
\hline Cowley et al. ${ }^{10}$ & N.r. & N.r. \\
\hline Deb $^{45}$ & Clinical features & N.r. \\
\hline Deb $^{44}$ & N.r. & N.r. \\
\hline Deb et al. ${ }^{46}$ & $\begin{array}{l}\text { Interview with patients and } \\
\text { carers, medical case-notes }\end{array}$ & Interview with patients and carers, medical case-notes \\
\hline Deb et al. ${ }^{47}$ & $\begin{array}{l}\text { Interview with subjects and } \\
\text { carers }\end{array}$ & Interview with subjects and carers \\
\hline Didden et al..$^{25}$ & Parent questionnaire & $\begin{array}{l}\text { Results from standardized instrument from child's } \\
\text { dossier }\end{array}$ \\
\hline Espie et al. ${ }^{29}$ & $\begin{array}{l}\text { Ratings by experienced } \\
\text { epileptologists }\end{array}$ & Vineland Adaptive Behavior Scales \\
\hline Fitzgerald et al..$^{20}$ & N.r. & N.r. \\
\hline Hilger et al. ${ }^{33}$ & N.r. & Attendance special school (relating to $\mathrm{IQ}<70$ ) \\
\hline Johnson et al. ${ }^{46}$ & Child neurologist & N.r. \\
\hline Jones et al. ${ }^{47}$ & Reports by teacher and parent & N.r. \\
\hline Koskentaus et al.11 & N.r. & Psychological assessment or case reports \\
\hline Lacey et al. ${ }^{48}$ & Epilepsy specialist & N.r. \\
\hline Lewis et al. ${ }^{24}$ & Parent interview & Cognitive tests administered by psychologists \\
\hline Matson et al. ${ }^{49}$ & Neurologist & N.r. \\
\hline Matsuura et al. ${ }^{50}$ & Self-developed 5-axial scheme & Medical records, interview patient and family \\
\hline Matsuura et al. ${ }^{32}$ & N.r. & Wechsler Adult Intelligence Scale-Revised \\
\hline Matthews et al. ${ }^{23}$ & $\begin{array}{l}\text { Data from epilepsy research } \\
\text { nurse, evaluated by doctors }\end{array}$ & Medical notes and records \\
\hline McGrother et al. ${ }^{21}$ & $\begin{array}{l}\text { Structured home interview with } \\
\text { carers by trained interviewers }\end{array}$ & Identified by Leicester Learning Disability Register \\
\hline Molteno et al..$^{51}$ & N.r. & N.r. \\
\hline Pawar et al..$^{52}$ & Case notes & N.r. \\
\hline Piazzini et al. ${ }^{53}$ & Patient interview & N.r. \\
\hline Reilly et al. $^{31}$ & Pediatric neurologists & Psychological assessment by educational psychologists \\
\hline Ring et al. ${ }^{54}$ & $\begin{array}{l}\text { Clinical notes or EEG and imaging } \\
\text { data }\end{array}$ & Mostly Wechsler Adults Intelligence Scale \\
\hline Roberts et al. ${ }^{55}$ & N.r. & N.r. \\
\hline Smith et al. ${ }^{56}$ & Neurologist & Psychologist \\
\hline Smith et al. ${ }^{26}$ & Neurologist & Psychologist \\
\hline Smith et al. ${ }^{57}$ & Neurologist & Psychologist \\
\hline
\end{tabular}


Table 1.2.6: (continued)

\begin{tabular}{|c|c|c|}
\hline \multirow[t]{2}{*}{ Study } & \multicolumn{2}{|l|}{ Measure } \\
\hline & Epilepsy & Level ID \\
\hline Steffenburg et al..$^{58}$ & N.r. & $\begin{array}{l}\text { Wechsler Intelligence Scale for Children, Griffiths' } \\
\text { Developmental Scales, Leiter international scale and } \\
\text { Raven Progressive Matrices }\end{array}$ \\
\hline Steffenburg et al. ${ }^{30}$ & $\begin{array}{l}\text { Interview with patients, parents, } \\
\text { nursing staff and teachers }\end{array}$ & $\begin{array}{l}\text { Wechsler Intelligence Scale for Children, Griffiths' } \\
\text { Developmental Scales, Leiter international scale and } \\
\text { Raven Progressive Matrices }\end{array}$ \\
\hline Turkistani et al. ${ }^{59}$ & Case notes & Case notes \\
\hline Turky et al. ${ }^{19}$ & Medical records & $\begin{array}{l}\text { Clinical and psychometric reports, Adaptive Behavior } \\
\text { Scales }\end{array}$ \\
\hline Tyrer et al. ${ }^{27}$ & $\begin{array}{l}\text { Structured home interview with } \\
\text { carers by trained interviewers }\end{array}$ & Identified by Leicester Learning Disability Register \\
\hline
\end{tabular}

N.r. = not reported 


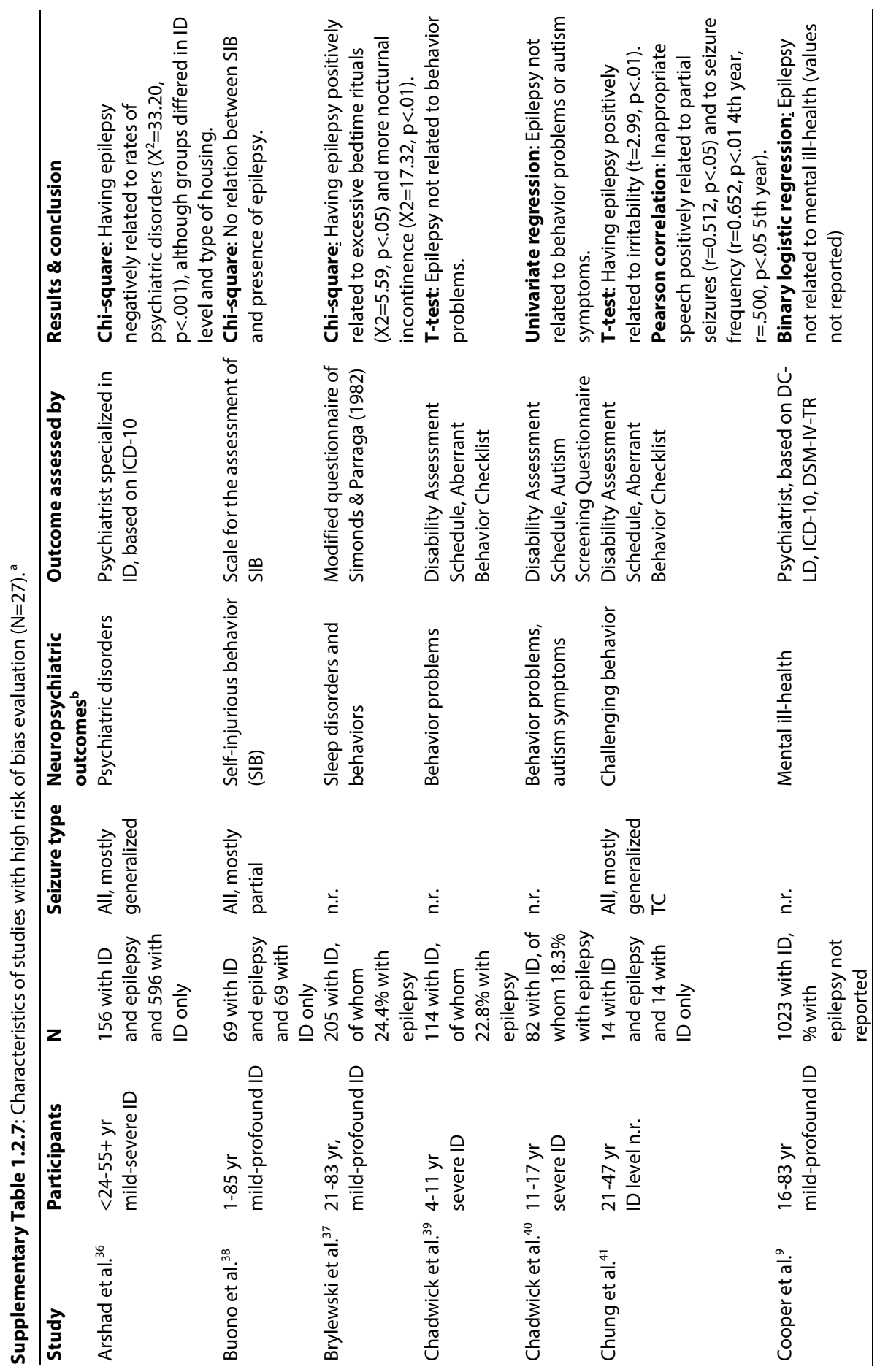




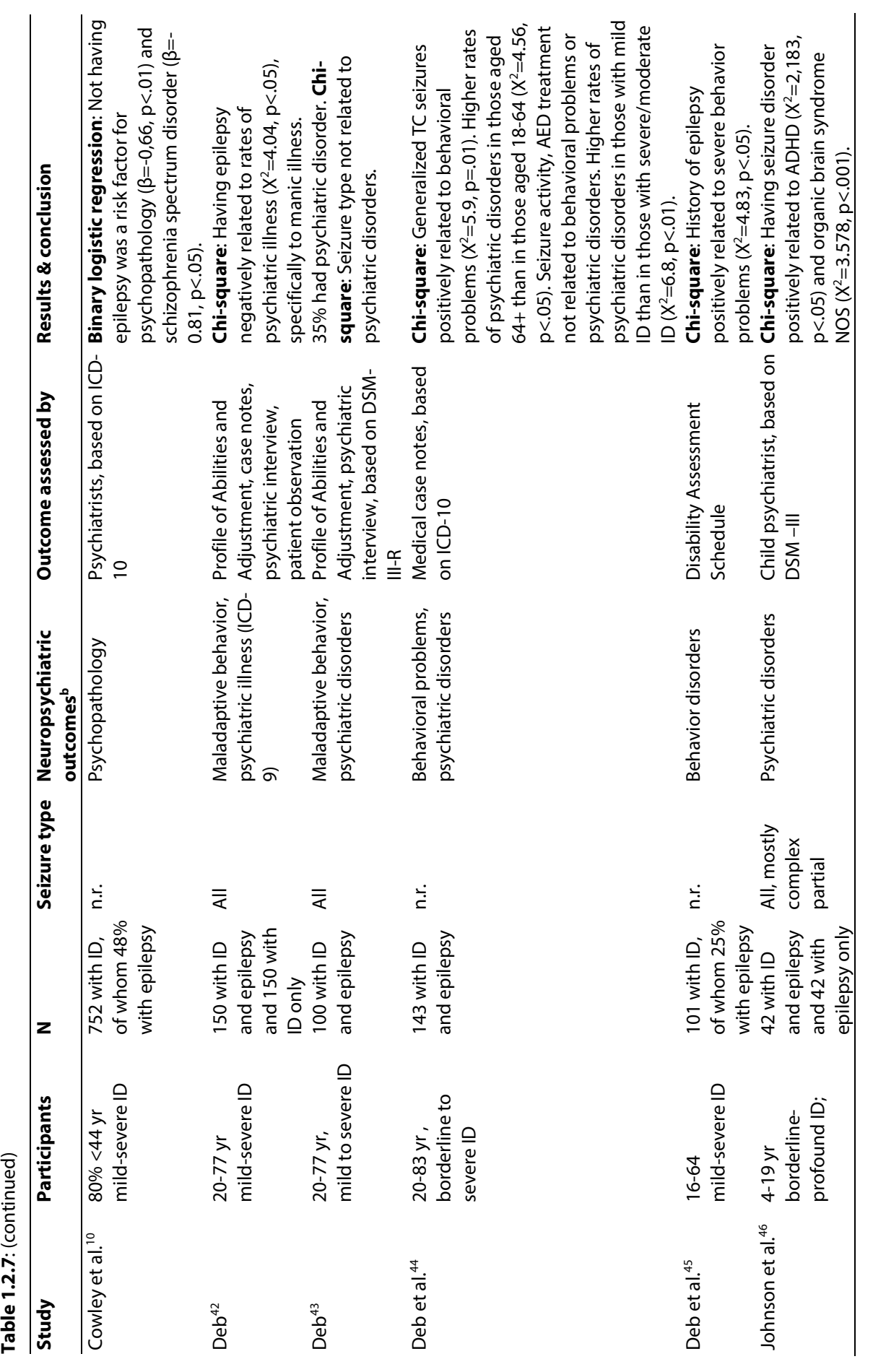




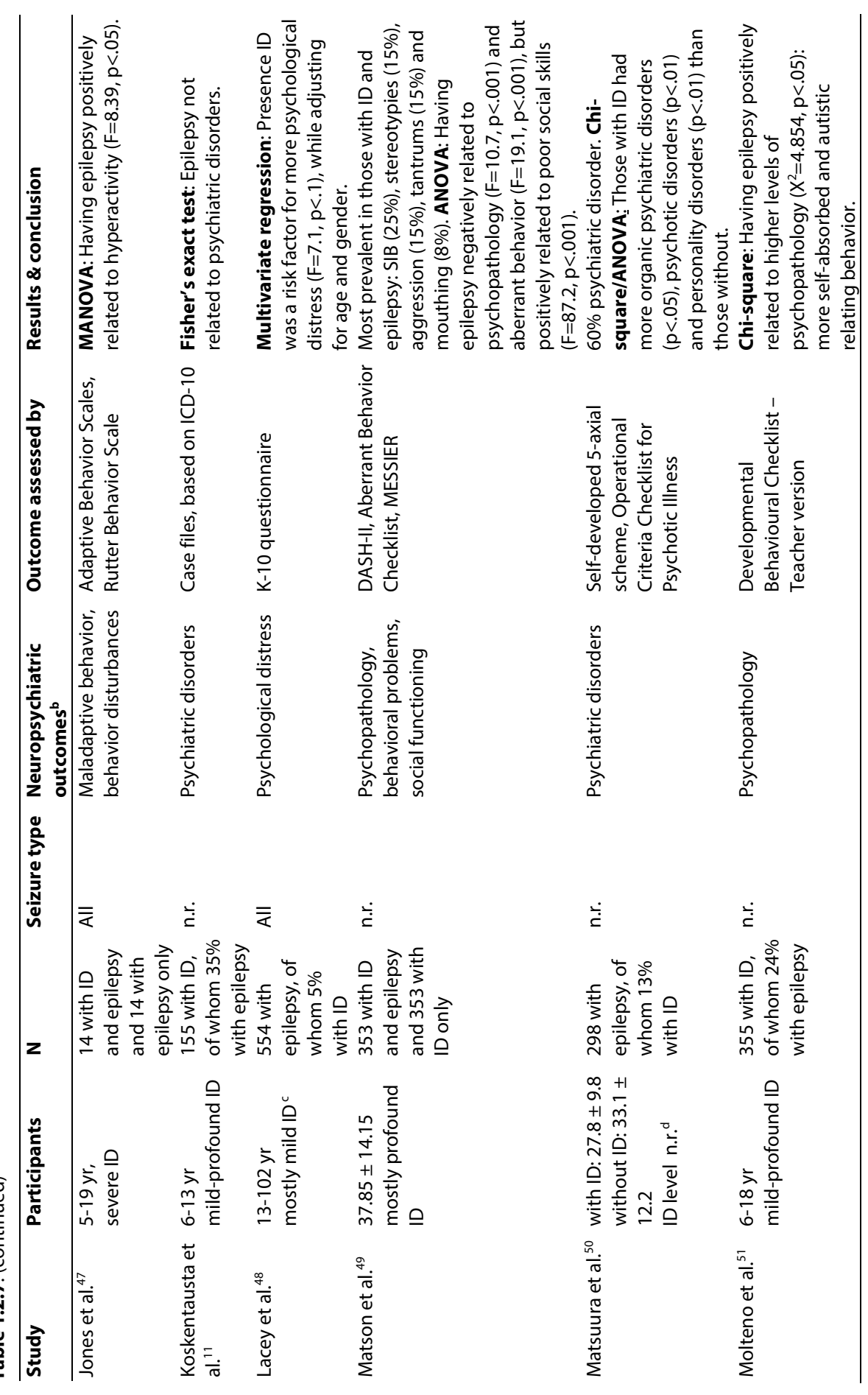




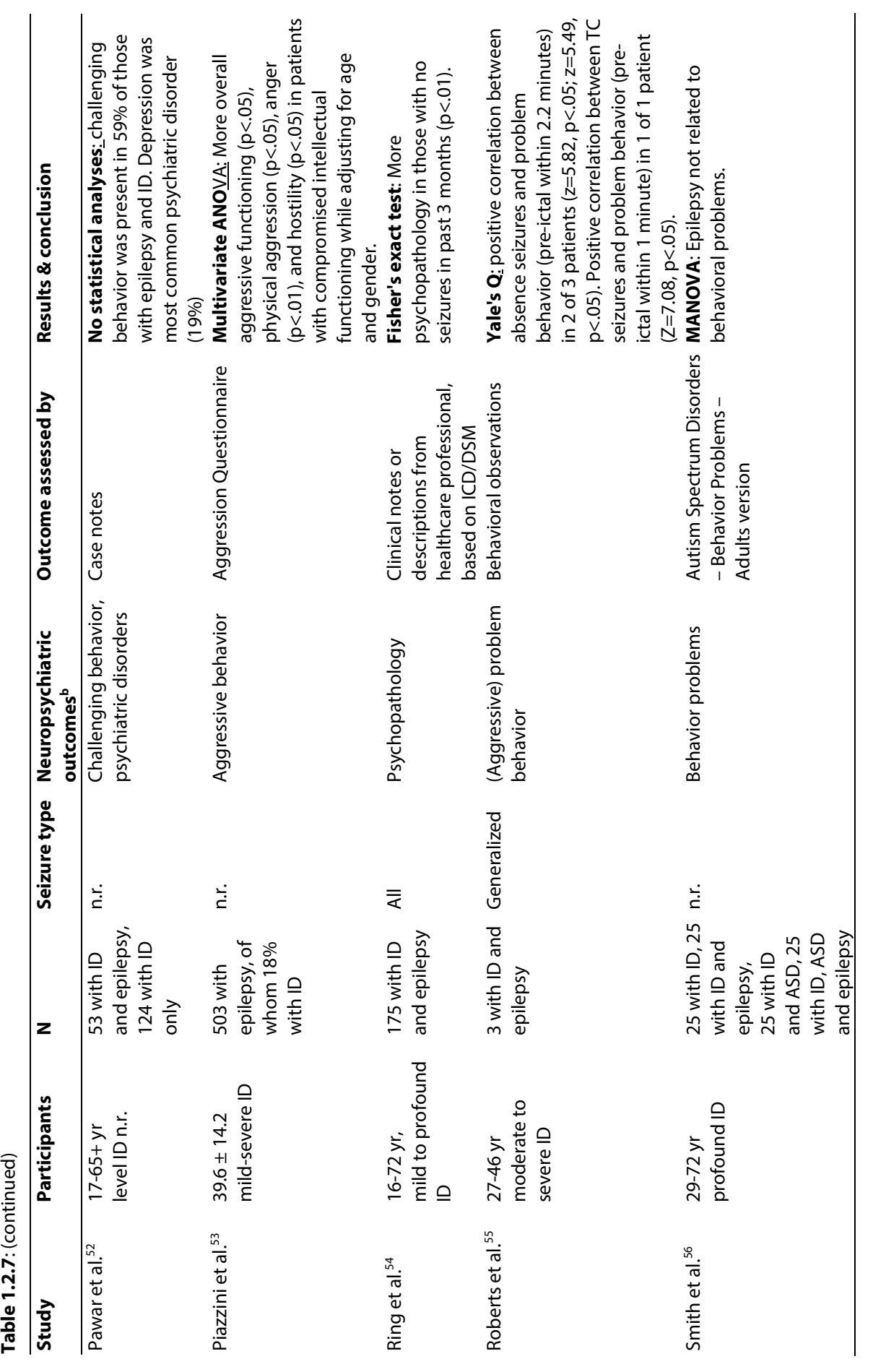




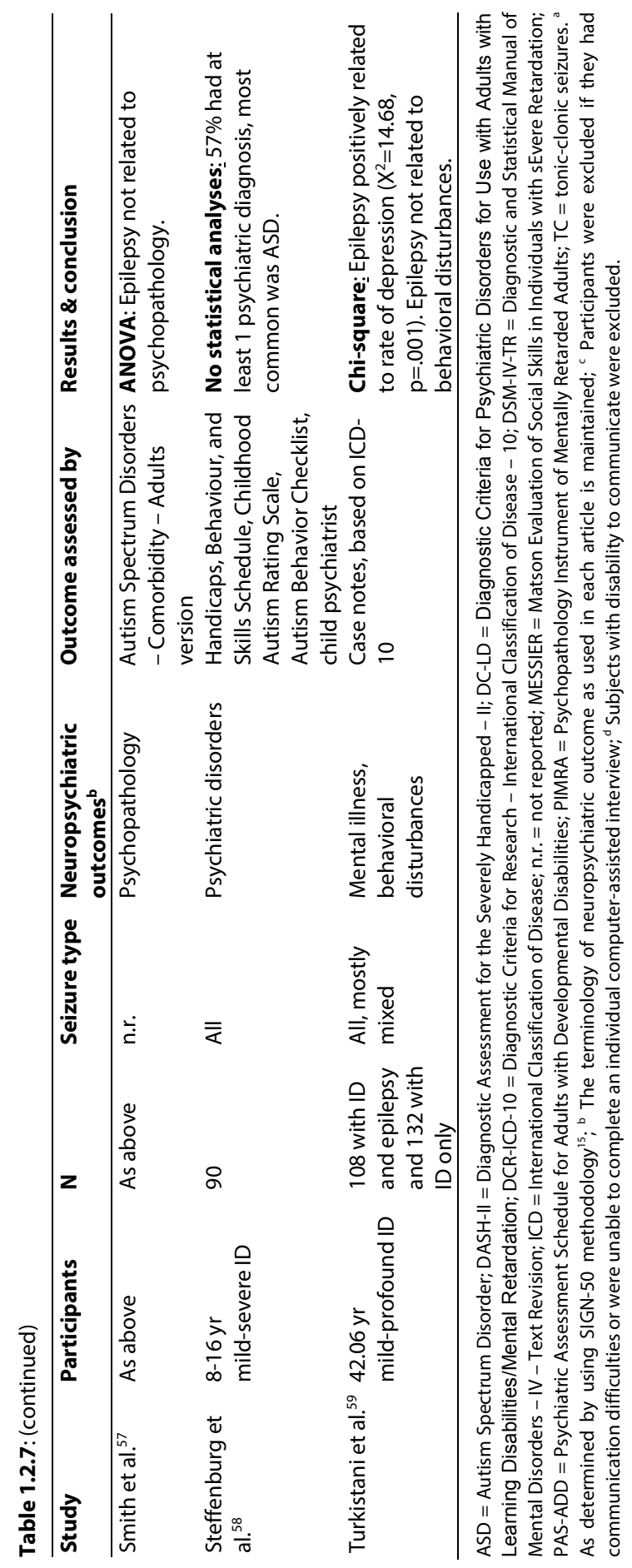




\section{Chapter 1.3}

General introduction - aim and outline 


\section{General introduction - aim and outline}

This thesis was part of a so called "twin"-PhD, that stresses the relevance of a multidisciplinary and holistic approach to the existing problems in care for people with ID and epilepsy. This thesis covers the medical perspectives. The dissertation on the psychological perspectives performed by Dr. Jans van Ool, behavioral scientist.

There are no strict national or international guidelines how to approach the complex problems of this population, when experiencing neuropsychiatric comorbidities.

With our joint PhD's we hope to lay the foundations for evidence based assessments, investigations and ultimately also treatment choices in this population.

We tried to visualize the pathway of considerations made in clinical practice when evaluating a patient with epilepsy, ID and neuropsychiatric problems. The different chapters in this thesis reflect the successive steps of this approach. 


\section{PATHWAY OF CONSIDERATIONS} IN PATIENTS WITH EPILEPSY, INTELLECTUAL DISABILITY \& NEUROPSYCHIATRIC PROBLEMS

\section{1}

What do we know about:

- Seizure \& epilepsy presentation - Level of intellectual disability - Intensity \& character of problems

\section{2 \\ Explanation from 1 perspective? \\ -Do we know the etiology?}

\section{3}

How do we assess the problems?

- Which instruments do we use based on validity/reliabilty

- Special issues / considerations in this complex group?

- Psychiatric symptoms?

04

Which specific factors should

we take into account?

\section{5}

\section{Extra attention needed} concerning medication?

- Specific anti-epileptic drugs?

-Known behavioral side-effects? 



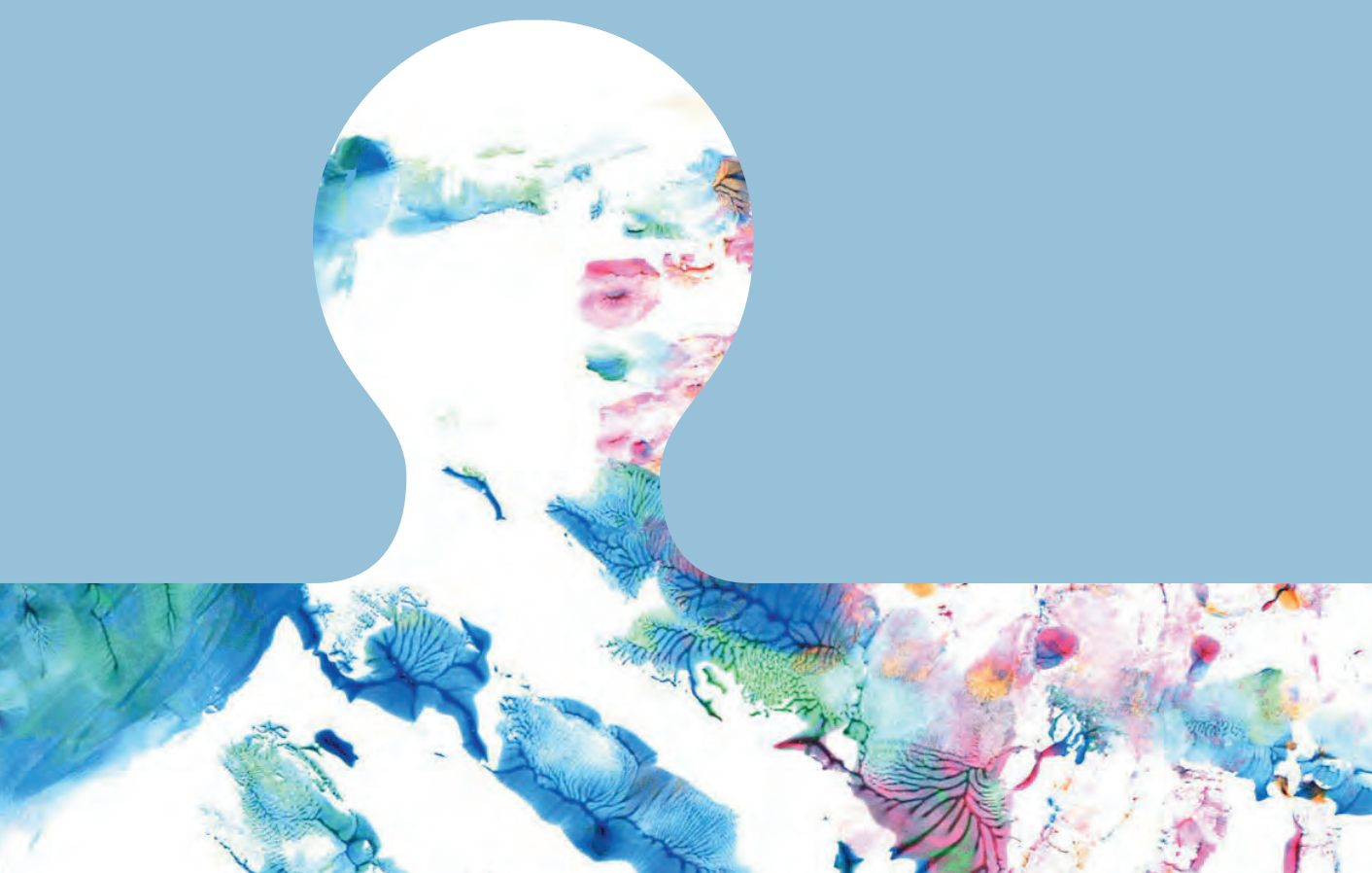

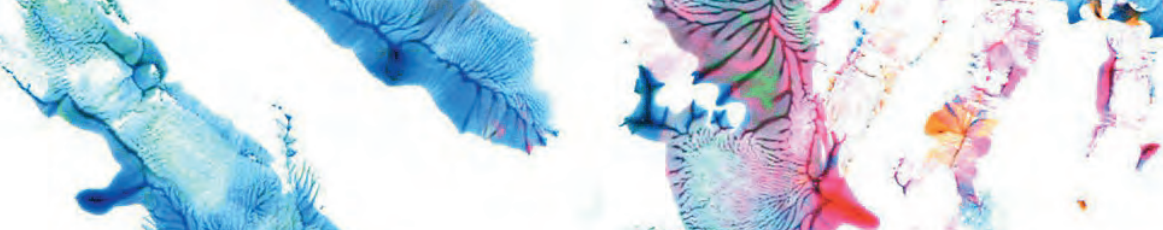

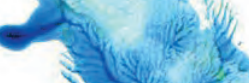

सेक्रा

का सहते

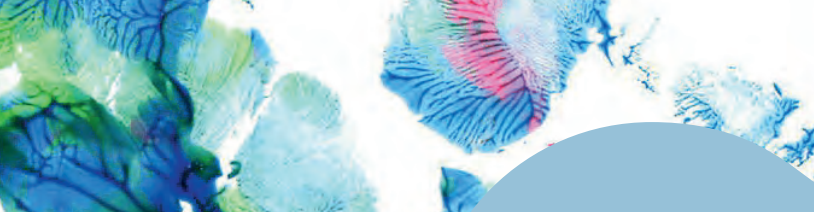

4

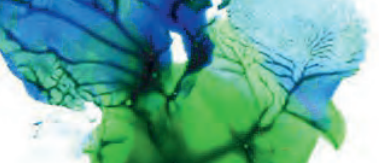

4 a

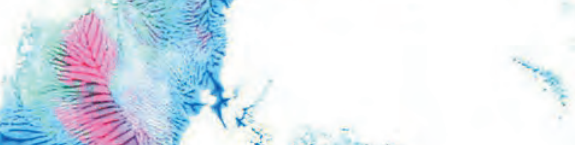

(1)

in $y-4$

7

here 35
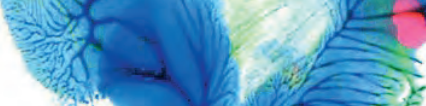

ant

If
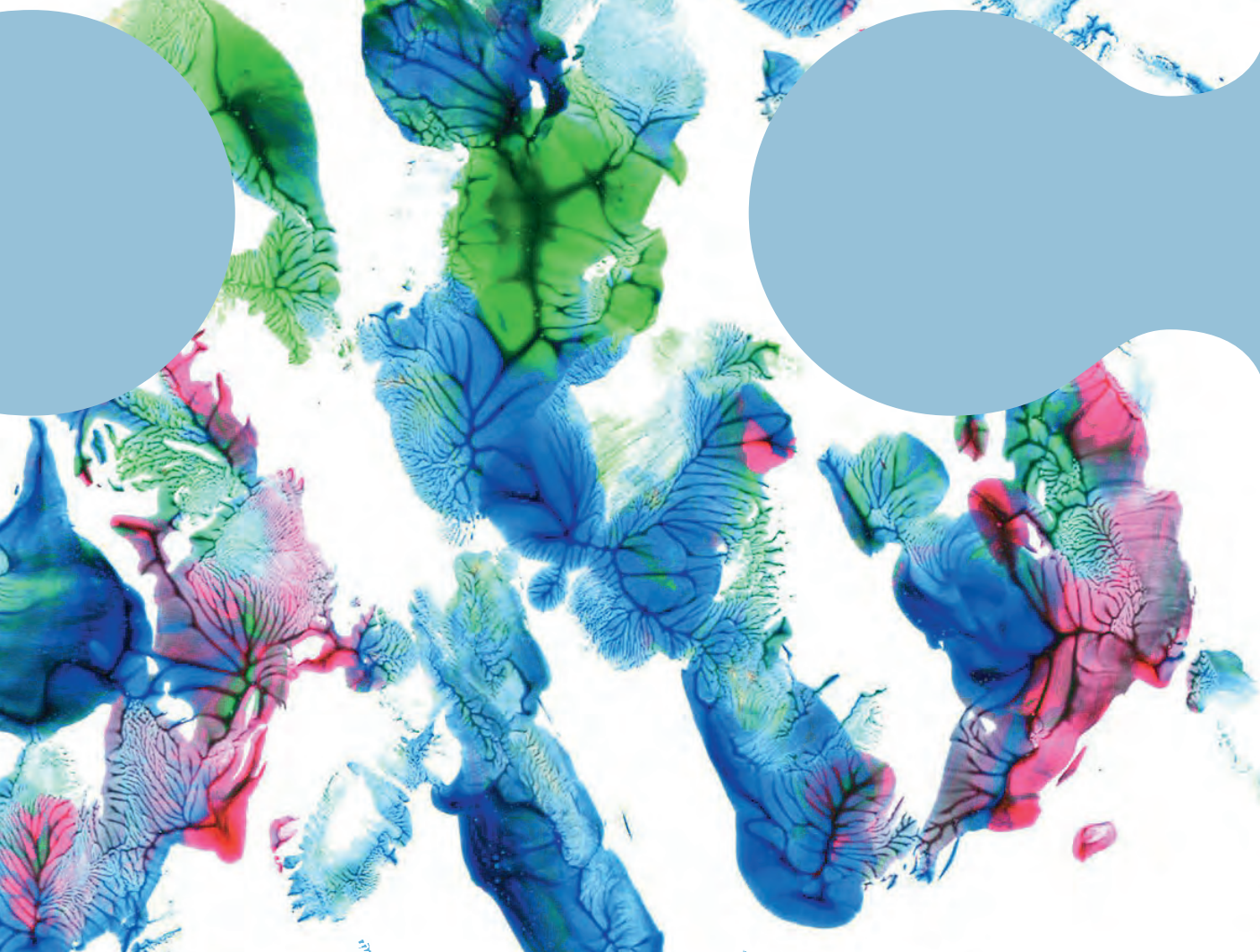


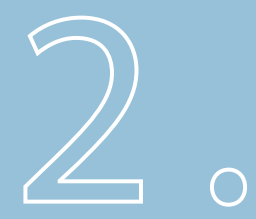

\section{Exome sequencing in patients with both epilepsy and ID}

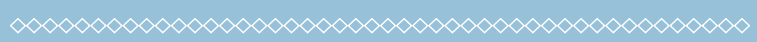

F.M. Snoeijen-Schouwenaars, J.S. van Ool, J.S. Verhoeven, P. van Mierlo, H.M.H. Braakman, E.E. Smeets, J. Nicolai, J. Schoots, M.W.A. Teunissen, R.P.W. Rouhl, I.Y. Tan, H.G. Yntema, H.G. Brunner, R. Pfundt, A.P. Stegmann, E-J. Kamsteeg, H.J. Schelhaas, M.H. Willemsen.

Epilepsia. 2019;60(1):155-64. 


\section{Abstract}

\section{Objective}

Epilepsy is highly prevalent among patients with intellectual disability (ID), and seizure control is often difficult. Identification of the underlying etiology in this patient group is important for daily clinical care. We assessed the diagnostic yield of whole exome sequencing (WES). In addition, we evaluated which clinical characteristics influence the likelihood of identifying a genetic cause and we assessed the potential impact of the genetic diagnosis on (antiepileptic) treatment strategy.

\section{Methods}

One hundred patients with both unexplained epilepsy and (borderline) ID (intelligence quotient 585 ) were included. All patients were evaluated by a clinical geneticist, a (pediatric) neurologist, and/or a specialist ID physician. WES analysis was performed in two steps. In step 1, analysis was restricted to the latest versions of ID and/or epilepsy gene panels. In step 2, exome analysis was extended to all genes (so-called full exome analysis). The results were classified according to the American College of Medical Genetics and Genomics guidelines.

\section{Results}

In 58 patients, the diagnostic WES analysis reported one or more variant(s). In 25 of the 100 patients, these were classified as (likely) pathogenic, in 24 patients as variants of uncertain significance, and in the remaining patients the variant was most likely not related to the phenotype. In 10 of 25 patients (40\%) with a (likely) pathogenic variant, the genetic diagnosis might have an impact on the treatment strategy in the future.

\section{Significance}

This study illustrates the clinical diagnostic relevance of WES for patients with both epilepsy and ID. It also demonstrates that implementing WES diagnostics might have impact on the (antiepileptic) treatment strategy in this population. Confirmation of variants of uncertain significance in (candidate) genes may further increase the yield. 


\section{Introduction}

Epilepsy is a neurological condition that is particularly common in patients with intellectual disability (ID). The prevalence of epilepsy among patients with ID is estimated to be around $22.2 \%$ (95\% confidence interval $=19.6-25.0)$, with prevalence rates increasing with severity of ID. ${ }^{1}$ These findings contrast with the rates of around $1 \%$ that are reported for the general European population. ${ }^{2}$

There is increased interest in understanding ID- and epilepsy- associated (neuropsychiatric) health risks from an etiological perspective. This interest is partly due to advanced genomic technologies now being able to identify a cause in an increasing proportion of patients with ID and in patients with epilepsy. These new diagnoses provide opportunities to delineate cause-specific characteristics and may lead to a greater understanding of comorbidities and prognosis. Furthermore, establishing a genetic diagnosis may even influence treatment choices, as has been demonstrated in patients with variants in the SCN1A, SCN8A, SLC2A1, and POLG genes.

Ideally, a recognizable phenotype will lead to the suspicion of a specific clinical diagnosis followed by confirmation with a specific diagnostic test, such as Sanger sequencing of the associated gene. ID and epilepsy, however, are extremely clinically and genetically heterogeneous, which challenges the search for an etiological diagnosis. In addition, the clinical spectrum of a specific genetic defect can be very broad. $^{3}$

Before the introduction of whole exome sequencing (WES), an etiological diagnosis could be established in about $40 \%$ of patients with previously unexplained developmental delay or ID, by using clinical examination and subsequent specific (DNA) diagnostic tests, high-resolution genome-wide chromosomal analysis, and screening for metabolic disorders. ${ }^{4-6}$ Recent studies have demonstrated the efficacy of detecting genetic alterations using a next generation sequencing panel approach or WES $^{7-12}$ in patients with epilepsy. Some of these studies also included patients with ID. The diagnostic yield varied between $30 \%$ and $70 \% .^{7-12}$

In this study, we specifically focused on patients with both epilepsy and (borderline) ID. This focus is in line with the white paper on the medical and social needs of people with epilepsy and intellectual disability of the International League Against Epilepsy ${ }^{13}$ that recommended action in investigation of etiology and mentioned that specific attention is needed to different aspects concerning adequate antiepileptic treatment in this population. 
We assessed the diagnostic yield in this group and evaluated which clinical characteristics determined the likelihood of identifying a genetic diagnosis. In addition, we discuss the potential consequences of the newly established genetic diagnosis for the treatment strategy.

\section{Materials and methods}

\subsection{Study population}

We retrospectively included 100 consecutive patients, both adults and children, with both epilepsy and (borderline) ID with an unknown cause. Inclusion started in January 2016. Enrollment was based on the clinical referral to our multidisciplinary outpatient clinic at the Academic Center for Epileptology at Kempenhaeghe, Heeze, The Netherlands or Maastricht University Medical Center, Maastricht, the Netherlands. All patients were evaluated by a clinical geneticist and a (pediatric) neurologist and/or specialist ID physician with specific expertise in this group of patients.

The study was assessed by the ethical committee of Maastricht University Medical Center (16-2-248.1) and was found to have no obligations regarding the Dutch Act on Medical Research in People.

\subsection{Inclusion criteria}

To be enrolled in this study, a patient had to meet all of the following criteria:

- Unexplained ID according to International Classification of Diseases, 10th revision ${ }^{14}$ and Diagnostic and Statistical Manual of Mental Disorders, 5th edition, ${ }^{15}$ defined as having both reduced intellectual functioning (intelligence quotient $<70$ ) and impaired adaptive abilities to cope with the daily demands of the social environment, manifesting during the developmental period of an individual. In addition, we included patients with borderline intellectual functioning (intelligence quotient $=70-85) .^{14}$

- Unexplained etiology of (active) epilepsy, according to the International League Against Epilepsy classification of Scheffer et al. ${ }^{16}$ This could be present as an associated feature; it was not necessary to have epilepsy as a main phenotypic feature.

- Parents and/or legal representative consented to anonymous inclusion of the clinical and molecular data for research purposes.

By not excluding patients with a positive family history, we were also able to identify focal familial forms of epilepsy. 


\subsection{Data collection}

Clinical data included demographics (age, sex, ethnicity), level of ID according to International Classification of Diseases, 10th revision ${ }^{14}$ Diagnostic and Statistical Manual of Mental Disorders, 5th edition, ${ }^{15}$ epilepsy and seizure type ${ }^{16}$ age at onset of epilepsy and ID, family history, seizure frequency, treatment of epilepsy, and results of brain magnetic resonance imaging. Furthermore, we also collected information about neuropsychiatric symptoms, dysmorphic features, major congenital anomalies, and previous (genetic) diagnostic tests.

A specialized neurologist (H.J.S.) rated the brain magnetic resonance imaging findings into three categories: normal, abnormal without epileptogenic focus, and abnormal with epileptogenic focus. The allocation was based on the likelihood that the lesions were responsible for the seizures presented in the individual patient. Therefore, if a lesion could, based on the magnetic resonance imaging location or characteristics, theoretically be epileptogenic but could not be linked to the seizure type of this specific patient, it was rated as abnormal but not epileptogenic.

\subsection{Study procedures}

Diagnostic WES was performed in all patients who remained undiagnosed by previous targeted DNA diagnostic tests (i.e., Sanger sequencing) on clinical indication. In most patients, chromosomal abnormalities were excluded in prior diagnostic evaluations. Recently, copy number variation analysis in WES data for the detection of chromosomal abnormalities became available. ${ }^{17}$ Therefore, genome-wide chromosomal analysis was not performed in all cases prior to WES.

Diagnostic exome sequencing was done at the Departments of Human Genetics of the Radboud University Medical Center Nijmegen and Maastricht University Medical Center, Maastricht, The Netherlands and performed essentially as described previously. ${ }^{18}$ It was preferentially done by a trio-based approach (patient + both parents) to facilitate the interpretation of variants.

In a first step of WES, only variants in known epilepsy (and/or ID) genes were considered by using a filter for specific panels in all patients (latest versions of epilepsy gene panel ${ }^{19}$ and/or ID gene pane ${ }^{20}$ ). The choice of a specific panel was based on clinical evaluation and indication; in nearly half of the patients (49/100) we opted for the epilepsy panel, as epilepsy was thought to be the primary/initial disorder. In 34 of 100 patients, both the epilepsy and ID panels were analyzed. In the remaining patients, only the ID gene panel was analyzed first, because in these cases, ID was thought to be the main feature. However, there is a substantial overlap between the ID and epilepsy gene panels (88.7\% of epilepsy candidate genes are 
covered in the ID panel, and $21.2 \%$ of ID candidate genes are covered in the epilepsy panel).

If the panel analysis did not reveal a pathogenic variant, the diagnostic exome sequencing was extended to the full exome; variants in all other genes were considered. Full exome analysis could only be performed among patients of whom parents or legal representatives provided written informed consent. Validation and segregation analysis of putative causative variants were done by Sanger sequencing.

\subsection{Interpretation of variants}

By the laboratories of genome diagnostics, variants were classified on the basis of the existing Dutch guidelines for evaluation of the pathogenicity of variants ${ }^{21}$ and the interpretation of sequence variants according to the American College of Medical Genetics and Genomics (ACMG) guidelines. ${ }^{22}$

The ACMG guidelines define 28 criteria (each with an assigned code) that address evidence such as population data, case-control analyses, functional data, computational predictions, allelic data, segregation studies and de novo observations. Each code is assigned a weight (stand-alone, very strong, strong, moderate, or supporting) and direction (benign or pathogenic). The combination of these evidence codes leads to the following five classifications: pathogenic, likely pathogenic, variant of uncertain significance (VUS), likely benign, or benign. ${ }^{22}$

\subsection{Statistical analyses}

Direct associations between clinical characteristics and the presence of a (likely) pathogenic variant were examined using the Pearson chi-square test or Fisher's exact test in case of low sample size in particular categories. We chose not to correct for multiple testing (Bonferroni) in this clinically orientated and relatively small study. The analyses were performed using SPSS Statistics version 23 software (IBM).

\section{Results}

Demographical and clinical characteristics of all patients with epilepsy and ID $(\mathrm{N}=100)$ are displayed in Table 2.1. Previous etiological research, such as DNA and/or chromosomal examination or a metabolic screening, was available in $96 \%$ of patients. An overview of the type of previous etiological investigations, related to the diagnostic yield found in this present study, is presented in Table 2.2. 
Table 2.1: Demographic and clinical characteristics ( $N=100)$.

\begin{tabular}{|c|c|}
\hline Characteristics & $\mathbf{N}$ \\
\hline Male & 55 \\
\hline Mean age, years (SD); & 24.1 years (16.2); range $2.8-67.6$ \\
\hline Children (< 18 years) & 47 \\
\hline Adults (>18 years) & 53 \\
\hline Caucasian & 98 ( 2 patients had African ethnicity) \\
\hline \multicolumn{2}{|l|}{ Level of ID } \\
\hline Borderline & 13 \\
\hline Mild & 35 \\
\hline Moderate & 13 \\
\hline Severe-Profound & 39 \\
\hline \multicolumn{2}{|l|}{ Onset of ID } \\
\hline Unknown & 2 \\
\hline Before onset epilepsy & 43 \\
\hline After onset epilepsy & 27 \\
\hline No differentiation & 28 \\
\hline \multicolumn{2}{|l|}{ Family history } \\
\hline ID & 17 \\
\hline Epilepsy & 18 \\
\hline Both epilepsy and ID & 13 \\
\hline No family history & 51 \\
\hline Unknown & 1 \\
\hline \multicolumn{2}{|l|}{ Age at onset of epilepsy } \\
\hline Neonatal period & 5 \\
\hline Infancy & 24 \\
\hline Childhood & 60 \\
\hline Adolescence & 9 \\
\hline Adulthood & 2 \\
\hline \multicolumn{2}{|l|}{ Epilepsy type $^{16}$} \\
\hline Focal & 15 \\
\hline Generalized & 12 \\
\hline Combined & 14 \\
\hline Unknown & 59 \\
\hline \multicolumn{2}{|l|}{ Seizure frequency } \\
\hline$>1 /$ day & 26 \\
\hline$\geq 1 /$ week, $<1 /$ day & 29 \\
\hline$\geq 1 /$ month, $<1 /$ week & 16 \\
\hline$<1 /$ month, $>1 /$ year & 14 \\
\hline No seizures under treatment & 15 \\
\hline \multicolumn{2}{|l|}{ Treatment } \\
\hline None & 4 \\
\hline 1 AED & 19 (1 also VNS) \\
\hline 2 AEDs & 30 ( 1 also VNS, 2 also KD) \\
\hline$\geq 3$ AEDs & 45 (6 also VNS, 1 also KD) \\
\hline Other (surgery or VNS, no AED) & 2 \\
\hline \multicolumn{2}{|l|}{ Brain MRI } \\
\hline Abnormal, with possible epileptogenic focus & 6 \\
\hline Abnormal, with no epileptogenic focus & 26 \\
\hline Normal & 54 \\
\hline Not performed & 14 \\
\hline
\end{tabular}


Table 2.1: (continued)

\begin{tabular}{|c|c|}
\hline Characteristics & $\mathbf{N}$ \\
\hline \multicolumn{2}{|l|}{ Dysmorphic features } \\
\hline Yes & 25 \\
\hline No & 73 \\
\hline Unknown & 2 \\
\hline \multicolumn{2}{|l|}{ Congenital anomalies } \\
\hline Yes & 8 \\
\hline No & 91 \\
\hline Unknown & 1 \\
\hline \multicolumn{2}{|l|}{ Previous etiological research } \\
\hline None & 4 \\
\hline DNA (specific) & 9 \\
\hline Chromosomal (genome-wide) & 32 \\
\hline DNA + chromosomal & 27 \\
\hline DNA + metabolic screening & 4 \\
\hline Chromosomal + metabolic screening & 2 \\
\hline DNA + chromosomal + metabolic screening & 22 \\
\hline \multicolumn{2}{|l|}{ WES panel } \\
\hline Epilepsy panel & 51 \\
\hline ID panel & 17 \\
\hline ID and epilepsy panel & 32 \\
\hline \multicolumn{2}{|l|}{ WES full exome } \\
\hline Yes & 56 \\
\hline \multicolumn{2}{|l|}{ Trio analysis } \\
\hline Yes & 66 \\
\hline \multicolumn{2}{|l|}{ Neuropsychiatric symptoms } \\
\hline No & 39 \\
\hline Yes & 61 \\
\hline Affective & 12 \\
\hline Psychotic & 1 \\
\hline Attention deficit disorder & 9 \\
\hline ASD & 22 \\
\hline Disruptive/impulse control & 13 \\
\hline Automutilation & 3 \\
\hline Sleep-wake disorder & 1 \\
\hline
\end{tabular}

$\mathrm{ID}=$ Intellectual Disability; AED = Anti-epileptic drugs; KD = Ketogenic diet; VNS = Vagus nerve stimulation; WES = Whole exome sequencing; $\mathrm{ASD}=$ autism spectrum disorder.

Table 2.2: Previous etiological investigations compared to current WES results.

\begin{tabular}{lrcc}
\hline Previous etiological investigations & N & \multicolumn{2}{c}{ Diagnostic yield current WES } \\
\cline { 3 - 4 } & & (Likely) pathogenic & VUS \\
\hline None & 4 & $50.0 \%$ & $25.0 \%$ \\
DNA (specific) & 9 & $11.1 \%$ & $33.3 \%$ \\
Chromosomal (genome-wide) & 32 & $18.8 \%$ & $21.9 \%$ \\
DNA + chromosomal & 27 & $22.2 \%$ & $18.5 \%$ \\
DNA + metabolic screening & 4 & $25.0 \%$ & $25.0 \%$ \\
Chromosomal + metabolic screening & 2 & $100.0 \%$ & $0.0 \%$ \\
DNA + chromosomal + metabolic screening & 22 & $31.8 \%$ & $31.8 \%$ \\
\hline
\end{tabular}

VUS $=$ variant of uncertain significance. 


\subsection{WES analyses}

In 66 of the 100 patients, WES analysis was trio-based. In the remaining 34, WES analysis was done in the index only, due to lack of consent or availability of DNA of both parents, necessary for the full exome analysis. If indicated and possible, additional segregation analysis in other family members was performed, such as in a single available parent, and other family members, both affected and unaffected. Initially, in 49 of the 100 patients analysis was restricted to the epilepsy gene panel ${ }^{19}$ and in 17 of the 100 patients the analysis was restricted to the ID panel. ${ }^{20}$ In 34 of the 100 patients, both the epilepsy and ID gene panels were analyzed. Extension of the analysis to the full exome was performed if the ID and/or epilepsy gene panels did not detect a (likely) pathogenic variant (56/100 patients), consent for full exome analysis was given, and DNA of both parents was available. We summarized the process of analysis in a flowchart (Figure 2.1).

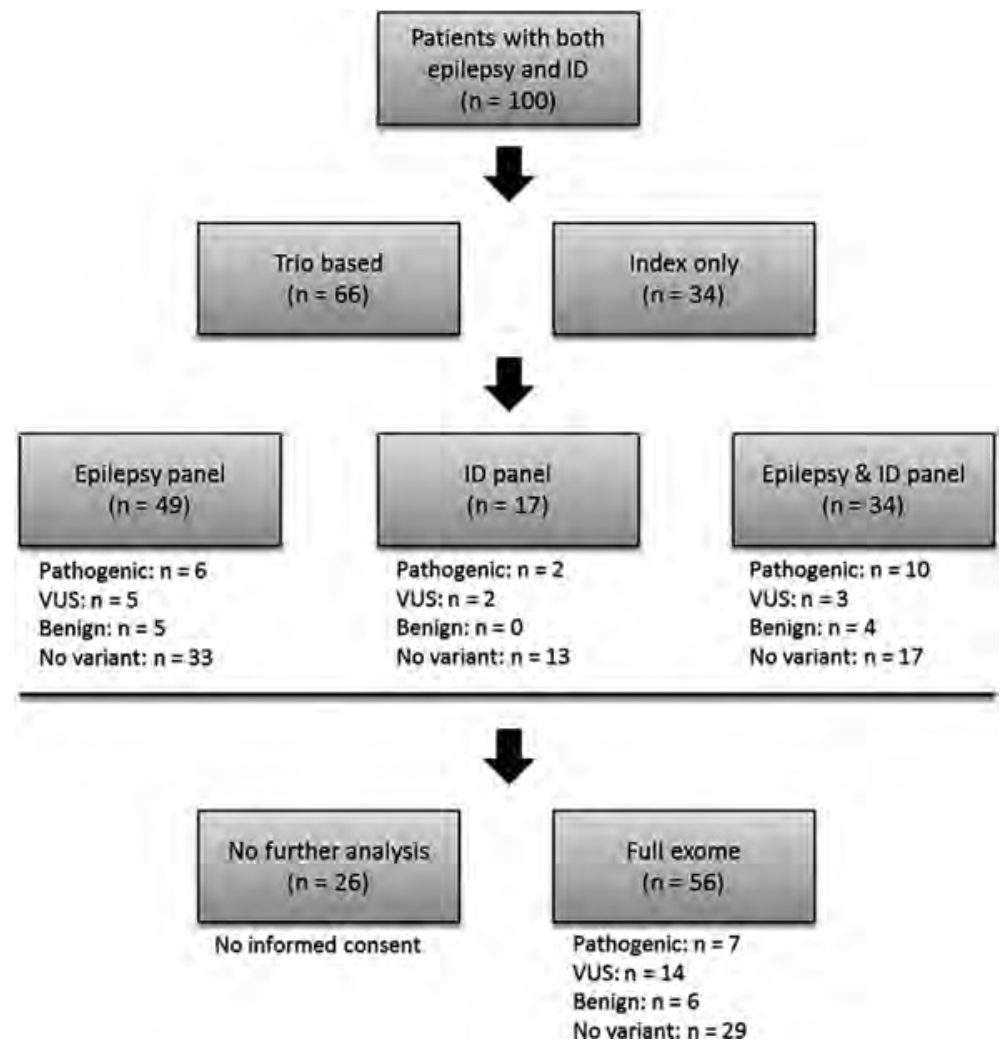

Figure 2.1: Flowchart of diagnostic analysis. ID, intellectual disability; VUS, variant of uncertain significance. 


\subsection{Diagnostic yield}

In 59 patients, one or more genetic variant(s) of interest were reported in the WES analysis, with either dominant ( $n=51$, mostly de novo), recessive $(n=2)$, or $X$-linked $(n=6)$ inheritance. In 25 of the 100 patients, these were classified as (likely) pathogenic, in 24 cases as VUS, and in the remaining cases the identified variant was classified as (likely) benign. See Table 2.3 for an overview of the diagnostic yield, including the genes involved. (Likely) pathogenic variants were found by panel analyses (epilepsy and/or ID panel) in $72 \%$ and the remaining $28 \%$ by additional full exome analysis. In four of nine patients who had WES with analysis of both the epilepsy and ID panels, a pathogenic variant in a specific ID gene that is not covered by the epilepsy panel was identified. A complete overview of the likely pathogenic variants and VUS/VUS in candidate genes is presented in Tables S2.1 and S2.2.

Table 2.3: Diagnostic yield of WES in patients $(N=100)$ with both epilepsy and ID of previously unknown cause.

\begin{tabular}{|c|c|c|c|}
\hline Interpretation of variants & $\mathbf{N}$ & Gene & $\begin{array}{l}\text { Panel/Full } \\
\text { exome analysis }\end{array}$ \\
\hline (Likely) pathogenic & 25 & $\begin{array}{l}\text { SCN1A (3x),DEPDC5, CASK, CACNA1A, BRAF, } \\
\text { TSEN54*, CDK13, PGAP3*, CLCN4, STXBP1, } \\
\text { SCN8A(2x),CHD2, KMT2D, FOXG1, TCF20, HERC2*, } \\
\text { NEXMIF, SLC13A5*, SLC6A1 (2x), NBEA, EHMT1, } \\
\text { PURA, del 16q24.3; del 6q21q22, KCNQ5 }\end{array}$ & $\begin{array}{l}\text { Panel: } 18 \\
\text { Full exome: } 7\end{array}$ \\
\hline VUS & 24 & $\begin{array}{l}\text { MACF1, BRAF, TSC1, SPTAN1 (2x), SPTAN1+ } \\
\text { THUMPD3 (same patient), CASP8AP2 + DEPDC5 } \\
\text { (same patient), MGA + TSPAN5 (same patient), } \\
\text { SPRED2, SAMD4A, CTNNA2, GLRA2, ABCC8+DNM1 } \\
\text { (same patient),CNNM2, CASK, NLGN4X, GRIN2A, } \\
\text { KIF1A, RAI1, ARGHAP35. }\end{array}$ & $\begin{array}{l}\text { Panel: } 10 \\
\text { Full exome: } 14\end{array}$ \\
\hline (Likely) benign or no variants & 51 & $\begin{array}{l}\text { GLDC**, KCNQ2, ABCC8, EHMT1, DYNC1H1, SMPD4, } \\
\text { SLC2A1+TNIK (same patient), RIMBP2,CLN3 }{ }^{* *}, \\
\text { No variants }(42 \mathrm{x})\end{array}$ & $\begin{array}{l}\text { Panel: } 3 \\
\text { Full exome: } 6\end{array}$ \\
\hline
\end{tabular}

* compound heterozygous/homozygous, ${ }^{* *}$ recessive disease. All other variants were heterozygous variants.

\subsection{Potential clinical indicators of pathogenic variants}

The analyses revealed that most clinical characteristics were not related to the WES outcome (see Table 2.4). The exception was that pathogenic variants were significantly more prevalent among patients with epilepsy originating after childhood $(p=0.001)$. 
Table 2.4: Associations between a likely causal variant and clinical characteristics.

\begin{tabular}{|c|c|c|c|}
\hline & (Likely) pathogenic & $\begin{array}{c}\text { VUS/benign/no } \\
\text { variant }\end{array}$ & p-value \\
\hline Age & & & $0.418^{\mathrm{a}}$ \\
\hline Children & $10(21.3 \%)$ & $37(78.7 \%)$ & \\
\hline Adults & $15(28.3 \%)$ & $38(71.7 \%)$ & \\
\hline Level of ID & & & $0.106^{\mathrm{a}}$ \\
\hline Borderline/Mild & $8(18.2 \%)$ & $36(81.8 \%)$ & \\
\hline Moderate/Severe & $17(32.7 \%)$ & $35(67.3 \%)$ & \\
\hline Onset of ID & & & $0.157^{\mathrm{a}}$ \\
\hline Before onset epilepsy & $14(32.6 \%)$ & $29(67.4 \%)$ & \\
\hline After onset epilepsy/no differentiation & $11(20.0 \%)$ & $44(80.0 \%)$ & \\
\hline Family history & & & $0.243^{b}$ \\
\hline No epilepsy/ID in family & $13(25.5 \%)$ & $38(74.5 \%)$ & \\
\hline ID or epilepsy in family & $11(31.4 \%)$ & $24(68.6 \%)$ & \\
\hline Both ID and epilepsy in family & $1(7.7 \%)$ & $12(92.3 \%)$ & \\
\hline Age of onset of epilepsy & & & $0.001^{\mathrm{b}}$ \\
\hline Infancy (<1 year) & $10(34.5 \%)$ & $19(56.5 \%)$ & \\
\hline Childhood & $8(14.0 \%)$ & $49(86.0 \%)$ & \\
\hline Adolescence-adulthood & $7(63.6 \%)$ & $4(36.4 \%)$ & \\
\hline Epilepsy type $^{16}$ & & & $0.585^{\mathrm{b}}$ \\
\hline Focal & $3(20.0 \%)$ & $12(80.0 \%)$ & \\
\hline Generalized & $2(16.7 \%)$ & $10(83.3 \%)$ & \\
\hline Combined & $2(14.3 \%)$ & $12(85.7 \%)$ & \\
\hline Unknown & $18(35.5 \%)$ & $41(69.5 \%)$ & \\
\hline Seizure frequency & & & $0.871^{\mathrm{b}}$ \\
\hline$>1 /$ day & $6(23.1 \%)$ & $20(76.9 \%)$ & \\
\hline$>1 /$ week & $9(31.0 \%)$ & $20(69.0 \%)$ & \\
\hline$>1 /$ month & $3(18.8 \%)$ & $13(81.3 \%)$ & \\
\hline$>1 /$ year & $4(28.6 \%)$ & $10(71.4 \%)$ & \\
\hline Seizure free (under treatment) & $3(20.0 \%)$ & $12(80.0 \%)$ & \\
\hline Brain MRI & & & $0.648^{b}$ \\
\hline Abnormal, epileptogenic & $2(33.3 \%)$ & $4(76.7 \%)$ & \\
\hline Abnormal, not epileptogenic & $8(30.8 \%)$ & $18(69.2 \%)$ & \\
\hline Normal & $11(20.4 \%)$ & $43(79.6 \%)$ & \\
\hline Not performed & $4(28.6 \%)$ & $10(71.4 \%)$ & \\
\hline Dysmorphia & & & $0.388^{\mathrm{a}}$ \\
\hline No & $17(23.3 \%)$ & $56(76.6 \%)$ & \\
\hline Yes & $8(32.0 \%)$ & $17(68.0 \%)$ & \\
\hline Congenital anomalies & & & $.999^{b}$ \\
\hline No & $22(24.2 \%)$ & $69(75.8 \%)$ & \\
\hline Yes & $2(25.0 \%)$ & $6(75.0 \%)$ & \\
\hline Neuropsychiatric symptoms & & & $.046^{\mathrm{b}}$ \\
\hline None & $12(30.8 \%)$ & $27(69.2 \%)$ & \\
\hline Affective (mood \& anxiety) & $6(50.0 \%)$ & $6(50.0 \%)$ & \\
\hline ASD & $4(18.2 \%)$ & $18(81.8 \%)$ & \\
\hline Other & $3(11.1 \%)$ & $24(88.9 \%)$ & \\
\hline
\end{tabular}

${ }^{\mathrm{a}}$ Pearson Chi-square Test; ${ }^{\mathrm{b}}$ Fisher Exact Test

Neuropsychiatric comorbidities were present in $62 \%$ of patients, with autism spectrum disorders being most frequently diagnosed (22\%). In addition, the presence 
of such comorbidities was also significantly related to detection of a causal variant with WES ( $p=0.046)$. More specifically, affective symptoms (i.e., depressive symptoms or anxiety) were significantly more prevalent in those with a pathogenic variant than in those without. For all other characteristics, no significant relationship to the outcome of WES was observed.

\subsection{Relevant findings for daily clinical care}

In five patients (20\%) with a (likely) pathogenic variant, we found variants with a potential consequence for antiepileptic drug treatment and clinical approach, that is, SCN1A $(3 \times)$ and SCN8A (2x). In Patient 4 (Table S2.1), in whom a SCN1A encephalopathy was not considered before this WES study, lamotrigine was reduced, and preliminary results show a positive effect, especially on the patient's behavior and mood. In another five (20\%) patients of the (likely) pathogenic group, we found variants with a possible consequence for daily clinical care, that is, in the genes CACNA1A, GRIN2A, SLC13A5, and SLC6A1 (2X). Whereas apart from SCN1A and SCN8A, no direct change in treatment strategy is to be expected yet, this might change in the future.

\section{Discussion}

In the current study of 100 patients with both epilepsy and ID, we identified a (likely) pathogenic variant in 25 and a VUS with possible relation to the phenotype in another 24. Eight patients had a VUS in a potential candidate gene for a phenotype with epilepsy and ID (such as SPRED2, GLRA2, and CTNNA2).

Yields in previous diagnostic cohort studies varied from $30 \%$ to $70 \%{ }^{7-12}$ and depended on the diagnostic strategy. Several studies used targeted next generation sequencing approaches with a fixed number of genes included.

The number of genes varied highly, and in addition, the filtering and interpretation of variants differed among the various studies. Therefore, the diagnostic yields of the studies are difficult to compare. In our study, the ACMG guidelines for classification of the variants were used. In these guidelines, the clinical information has a relatively low weight in the interpretation of variants. We identified several variants (variants in TSEN54, PGAP3, CLCN4, NBEA, and TCF20, see Table S2.2) that would be classified as likely pathogenic from a clinical point of view but do not strictly fulfill the criteria for pathogenicity according to the ACMG guidelines, for example, because functional evidence is lacking. If we would add these variants to the 
total yield, this would sum up to $30 \%$. Therefore, application of the ACMG criteria will likely have led to an underestimation of the clinical diagnostic value. However, it is inevitable to use stringent criteria as in the ACMG guidelines when making use of untargeted testing of large numbers of genes, rather than specifically testing one or a small number of genes based on a distinct phenotype.

The yield in this study may have been even higher if all patients had a full trio-based exome analysis, but as in regular daily clinical practice, full exome analysis is not always possible due to lack of consent or availability of DNA of one or both of the parents.

In our cohort, clinical characteristics of patients, such as congenital anomalies or dysmorphic features, seemed mostly unrelated to the WES outcome. This indicates that patient characteristics cannot predict the likelihood that WES will identify a causal variant for the phenotype. Therefore, genetic diagnostic investigation by WES should be considered in all patients with both unexplained epilepsy and ID. A (likely) pathogenic variant was more prevalent in the group of patients with a later onset of epilepsy (after childhood). This observation is possibly due to our multidisciplinary clinic being situated in a tertiary epilepsy center. In this setting, those patients with an obvious epilepsy syndrome originating early in life, such as Dravet syndrome or epileptic encephalopathy with continuous spike and wave during sleep, are likely to be diagnosed at an earlier stage by targeted DNA diagnostic testing.

Remarkably, the number of patients with an "unknown" epilepsy type is notably high in our cohort. This could be partly explained by the average age of the patients at the time of study (24 years) in combination with the finding that most patients suffered from epilepsy from an early age and the seizure semiology changed throughout the years.

Although there is a substantial overlap between the epilepsy and ID gene panels, it is important to realize that several genes that could potentially be associated with both phenotypes are not necessarily included in both panels. For example, epilepsy can be part of the phenotype in a small subset of the patients with a specific mutation but is not recognized as a distinctive feature of this mutation. In four cases, (likely) pathogenic variants were identified in specific ID genes that are not covered by the epilepsy panel, and thus would have been missed if only the epilepsy panel was analyzed (eg, Patient 8 in Table S2.1). Conversely, we could have missed some of the variants when the analysis would have been restricted to the ID panel, for example a variant in SLC6A1, as this gene was not included in the ID gene panel (see Patient 24 in Table S2.1). It is also possible that the epilepsy and ID have separate causes in a specific individual, which can be missed if the analysis is restricted to one of both panels. We therefore recommend analysis of both panels, especially when a full 
exome analysis is not possible or not consented to by the parents/legal representative(s). We would not recommend analysis of the total exome immediately, because of the chance of unsolicited findings and higher chance of detecting variants of uncertain significance, and because a substantial number of patients will be diagnosed by the panel analyses.

Although we did not find any unsolicited findings in our cohort, full exome analysis is accompanied by an increased risk of $1 \%-2 \%$ for possible unsolicited findings and has a higher incidence of identification of unknown variants. Despite this, full exome analysis also has the advantage of the possible identification of novel or very recently discovered new genes for phenotypes with both ID and epilepsy that have not yet been included in current panel releases. In addition, full exome analysis allows a genome-wide analysis for chromosomal copy number variations and can thereby largely replace separate chromosomal analysis by array analysis. ${ }^{23}$

Despite the high yield of WES, it is important to realize that not all genetic causes of ID and epilepsy can be detected by this approach. WES will not detect trinucleotide repeats, as for example a CGG repeat expansion in the FMR1 gene in fragile $X$ syndrome. In addition, balanced chromosomal rearrangements, such a ring chromosome 20 causing epilepsy, will not be detected using WES.

This study is in line with the report "Listening for a Change" that was published by the International League Against Epilepsy Task Force on ID and the International Bureau for Epilepsy. ${ }^{13,24}$ To optimize the genetic investigations as well as communication, all studies were performed and evaluated in a multidisciplinary setting at an outpatient clinic that is dedicated to the care of patients with ID and epilepsy. In line with the philosophy of this guideline, we also considered and discussed whether the outcome of the genetic studies might potentially guide treatment options (in the future) of the individual patient.

For 10 of the 25 patients (40\%) with a (likely) pathogenic variant, the new diagnosis might have, according to the literature, a consequence for antiepileptic treatment. ${ }^{25-29}$

For example, in patients with Dravet syndrome due to an SCN1A mutation, there is evidence for the efficacy of stiripentol, based on two randomized controlled trials (Chiron et al. ${ }^{25}$ and STICLO study group [not published]), and there are medications to avoid, especially lamotrigine, phenytoin, and carbamazepine. ${ }^{26}$

There is also literature suggesting that patients with SCN8A gain of function mutations have been shown to respond well to high-dose phenytoin. ${ }^{27}$ In addition, there is literature suggesting that for some patients with a GRIN2A missense mutation, the $N$-methyl-D-aspartate receptor blocker memantine should be considered ${ }^{28,29}$ and that finding a mutation in the CACNA1A, ${ }^{30}$ 
SLC13A5,,$^{31,32}$ and SLC6A1 $(2 \times)^{33}$ genes might guide antiepileptic treatment options in the future. For these genes, additional functional studies in a diagnostic setting will be necessary to delineate the full potential of personalized medicine.

Our study highlights the diagnostic relevance of WES for patients with both epilepsy and ID in clinical practice. Prior studies have been performed in a more selected group, that is, patients with epileptic encephalopathy, and with different inclusion criteria, for example, exclusion of patients with positive family history and/or structural anomalies. ${ }^{12,34}$ Because of the design of our study, we also identified patients with ID syndromes with epilepsy as an associated feature, but not necessary as the main feature. In addition, by not excluding patients with a positive family history, we were also able to identify focal familial forms of epilepsy, such as epilepsy caused by a mutation in DEPDC5. A limitation of our study is that our population was biased not only by the referral bias to a tertiary clinic but also by an almost exclusively Caucasian study population.

However, our study also demonstrates that implementing WES diagnostics might be relevant for the treatment strategy in this complex population.

The VUS that we identified in novel candidate genes for epilepsy and ID such as GLRA2, SAMD4A, and MACF1 warrant validation in future studies, such as functional studies and studies in larger patient groups, to confirm that these candidate genes are associated with epilepsy and ID and further delineate the related phenotype. 


\section{References}

1. Robertson J, Hatton C, Emerson E, et al. Prevalence of epilepsy among people with intellectual disabilities: a systematic review. Seizure. 2015;29:46-62.

2. Forsgren L, Beghi E, Oûn A, et al. The epidemiology of epilepsy in Europe-a systematic review. Eur J Neurol. 2005;12:245-53.

3. Mefford HC, Batshaw ML, Hoffman EP. Genomics, intellectual disability, and autism. N Engl J Med. 2012;366:733-43.

4. Leonard $\mathrm{H}$, Wen $\mathrm{X}$. The epidemiology of mental retardation: challenges and opportunities in the new millennium. Ment Retard Dev Disabil Res Rev. 2002;8:117-34.

5. Ropers HH. Genetics of early onset cognitive impairment. Annu Rev Genomics Hum Genet. 2010;11:161-87.

6. Topper S, Ober C, Das S. Exome sequencing and the genetics of intellectual disability. Clin Genet. 2011;80:117-26.

7. Della Mina E, Ciccone R, Brustia F, et al. Improving molecular diagnosis in epilepsy by a dedicated high-throughput sequencing platform. Eur J Hum Genet. 2015;23:354-62.

8. Lemke JR, Riesch E, Scheurenbrand T, et al. Targeted next generation sequencing as a diagnostic tool in epileptic disorders. Epilepsia. 2012;53:1387-98.

9. Veeramah KR, Johnstone L, Karafet TM, et al. Exome sequencing reveals new causal mutations in children with epileptic encephalopathies. Epilepsia. 2013;54:1270-81.

10. Orsini A, Zara F, Striano P. Recent advances in epilepsy genetics. Neurosci Lett. 2018;667:4-9.

11. Møller RS, Dahl HA, Helbig I. The contribution of next generation sequencing to epilepsy genetics. Expert Rev Mol Diagn. 2015;15:1531-8.

12. Epi4K Consortium, Epilepsy Phenome/Genome Project, Allen AS. De novo mutations in epileptic encephalopathies. Nature. 2013;501:217-21.

13. Kerr M, Linehan C, Thompson R, et al. A white paper on the medical and social needs of people with epilepsy and intellectual disability: the Task Force on Intellectual Disabilities and Epilepsy of the International League Against Epilepsy. Epilepsia. 2014;55:1902-6.

14. World Health Organization. The ICD-10 classification of mental and behavioural disorders: clinical descriptions and diagnostic guidelines. Geneva, Switzerland:World Health Organization; 1992.

15. American Psychiatric Association. Diagnostic and statistical manual of mental disorders. 5th ed. Arlington, VA: American Psychiatric Publishing; 2013.

16. Scheffer IE, Berkovic S, Capovilla G, et al. ILAE classification of the epilepsies: position paper of the ILAE commission for classification and terminology. Epilepsia. 2017;58:512-21.

17. Hehir-Kwa JY, Pfundt R, Veltman JA. Exome sequencing and whole genome sequencing for the detection of copy number variation. Expert Rev Mol Diagn. 2015;15:1023-32.

18. Neveling K, Feenstra I, Gilissen C, et al. A post-hoc comparison of the utility of sanger sequencing and exome sequencing for the diagnosis of heterogeneous diseases. Hum Mutat. 2013;34:1721-6.

19. Radboud UMC. Epilepsy gene panel DG 2.9/DG 2.10. 2016. Available at: https://issuu.com/radboudumc/docs/epilepsy_20pane I_20dg29-dg210?e $=28355229 / 55547221$. Accessed February 17, 2017.

20. Radboud UMC. Intellectual disability gene panel DG 2.7/DG 2.8. 2016. Available at: https://issuu.com/radboudumc/docs/intel lectual_20disability_20dg29-dg?e $=28355229 / 55547999$. Accessed February 17, 2017.

21. Wallis $Y$, Payne $S$, McAnulty $C$, et al. Practice guidelines for the evaluation of pathogenicity and the reporting of sequence variants in clinical molecular genetics. Association for Clinical Genetic Science and the Dutch Society of Clinical Genetic Laboratory Specialists; 2013. Available at: http://www.acgs.uk.com/media/774853/evaluation_and_reporting_of_sequence_variants_bpgs_jun e_2013_-_finalpdf.pdf. Accessed February 17, 2017.

22. Richards S, Aziz N, Bale S, et al. Standards and guidelines for the interpretation of sequence variants: a joint consensus recommendation of the American College of Medical Genetics and Genomics and the Association for Molecular Pathology. Genet Med. 2015;17:405-23. 
23. Pfundt R, Del Rosario $M$, Vissers LE, et al. Detection of clinically relevant copy-number variants by exome sequencing in a large cohort of genetic disorders. Genet Med. 2017;19:667-75.

24. Kerr M, Thompson R, Linehan C, et al. Listening for a change, medical and social needs of people with epilepsy and intellectual disability: a joint report of the International League Against Epilepsy Task Force on Intellectual Disabilities and the International Bureau for Epilepsy. Epilepsia. 2014;55:1902-6.

25. Chiron C, Marchand MC, Tran A, et al. Stiripentol in severe myoclonic epilepsy in infancy: a randomised placebo-controlled syndrome-dedicated trial. STICLO study group. Lancet. 2000;356:1638-42.

26. Guerrini $\mathrm{R}$, Dravet $\mathrm{C}$, Genton $\mathrm{P}$, et al. Lamotrigine and seizure aggravation in severe myoclonic epilepsy. Epilepsia. 1998;39:508-12.

27. Boerma RS, Braun KP, van den Broek MP, et al. Remarkable phenytoin sensitivity in 4 children with SCN8A-related epilepsy: a molecular neuropharmacological approach. Neurotherapeutics. 2016;13:192-7.

28. Pierson TM, Yuan H, Marsh ED, et al. GRIN2A mutation and early-onset epileptic encephalopathy: personalized therapy with memantine. Ann Clin TransI Neurol. 2014;1:190-8.

29. von Stülpnagel C, Ensslen M, Møller RS, et al. Epilepsy in patients with GRIN2A alterations: genetics, neurodevelopment, epileptic phenotype and response to anticonvulsive drugs. Eur J Paediatr Neurol. 2017;21:530-41.

30. Byers HM, Beatty CW, Hahn SH, et al. Dramatic response after lamotrigine in a patient with epileptic encephalopathy and a de novo CACNA1A variant. Pediatr Neurol. 2016;60:79-82.

31. Hardies K, de Kovel CG, Weckhuysen S, et al. Recessive mutations in SLC13A5 result in a loss of citrate transport and cause neonatal epilepsy, developmental delay and teeth hypoplasia. Brain. 2015;138:3238-50.

32. Klotz J, Porter BE, Colas C, et al. Mutations in the Na+/citrate cotransporter NaCT (SLC13A5) in pediatric patients with epilepsy and developmental delay. Mol Med. 2016;22:310-21.

33. Carvill GL, McMahon JM, Schneider A, et al. Mutations in the GABA transporter SLC6A1 cause epilepsy with myoclonic-atonic seizures. Am J Hum Genet. 2015;96:808-15.

34. Hamdan FF, Myers CT, Cossette $P$, et al. High rate of recurrent de novo mutations in developmental and epileptic encephalopathies. Am J Hum Genet. 2017;101:664-85. 


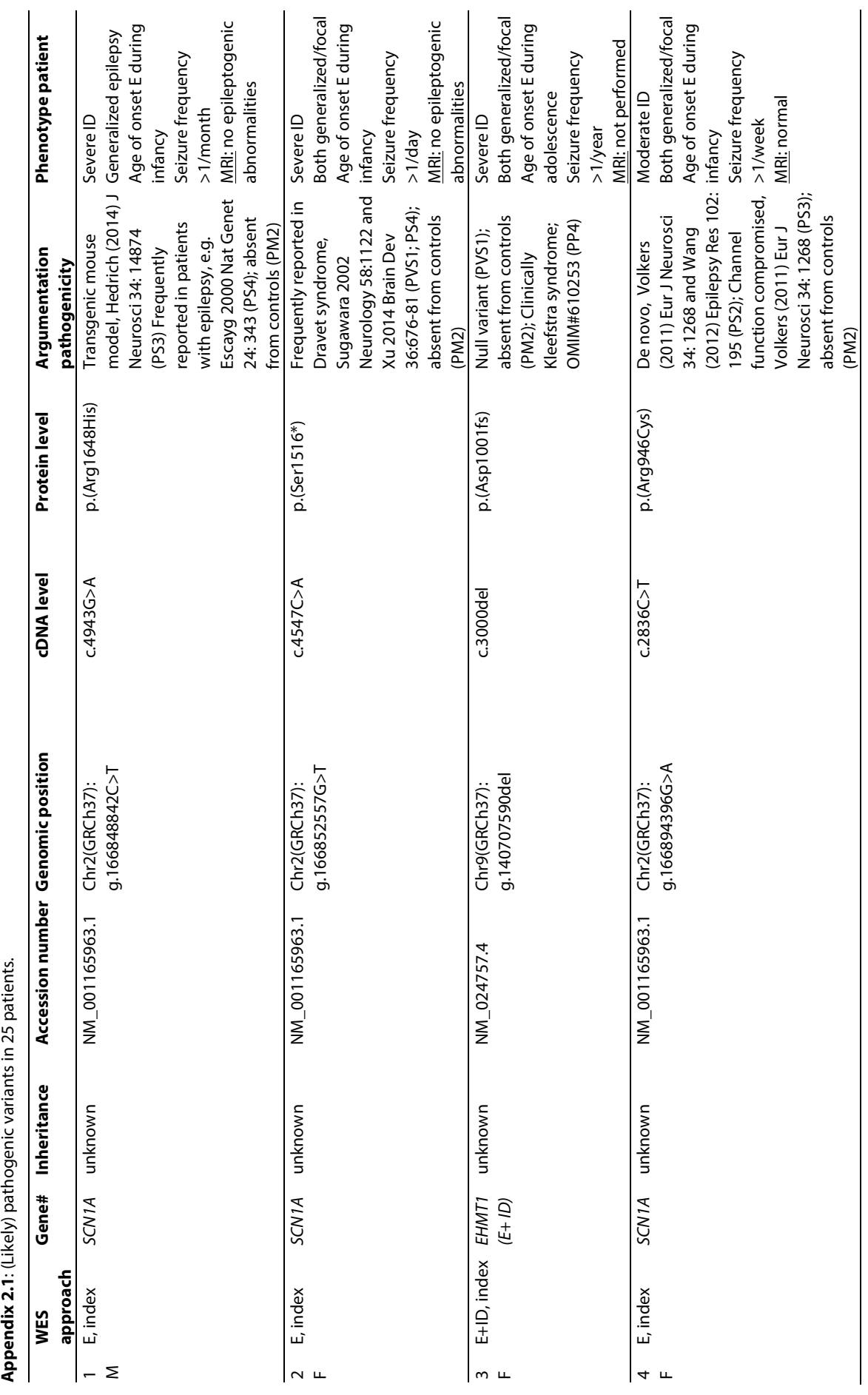




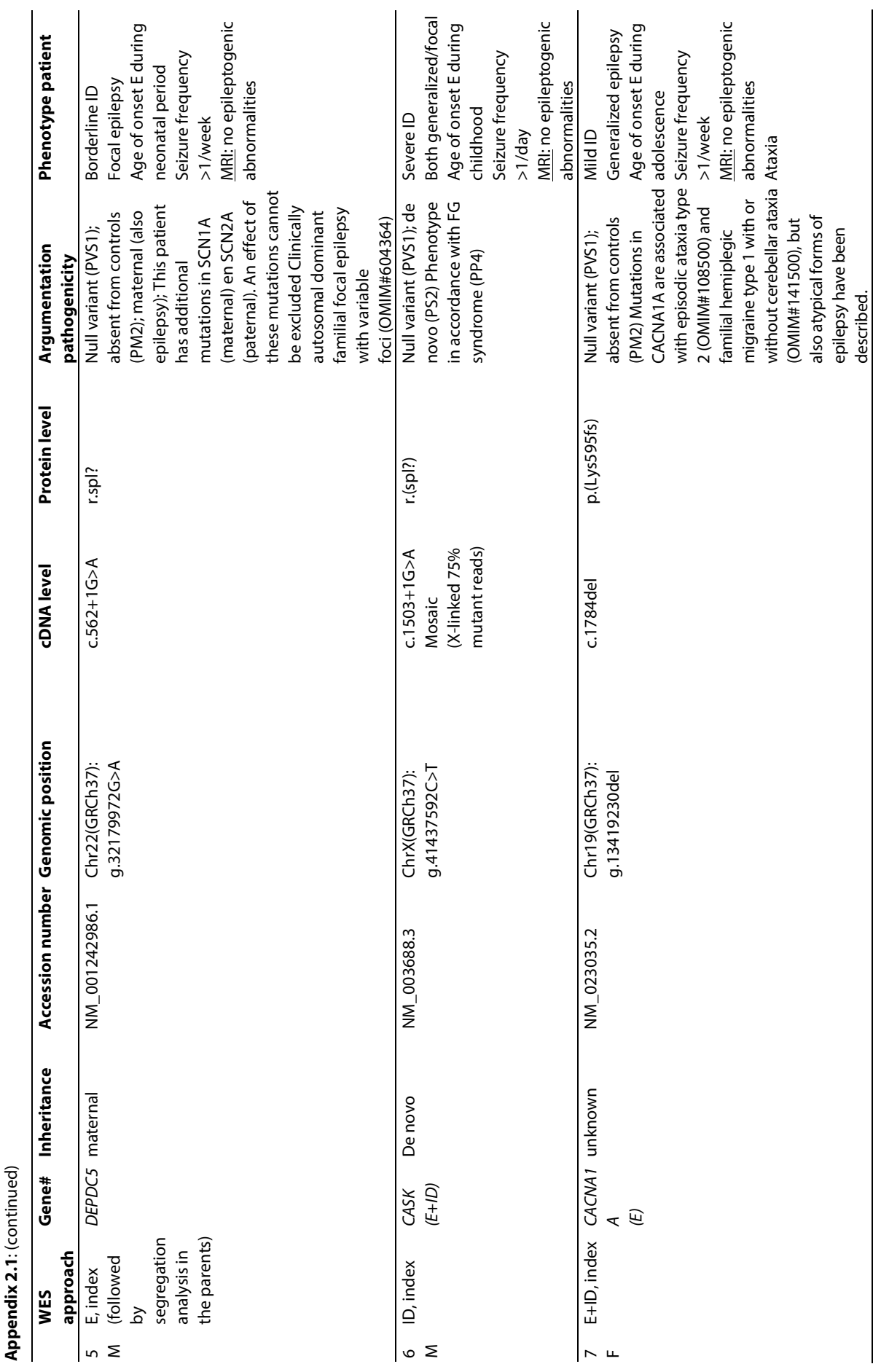




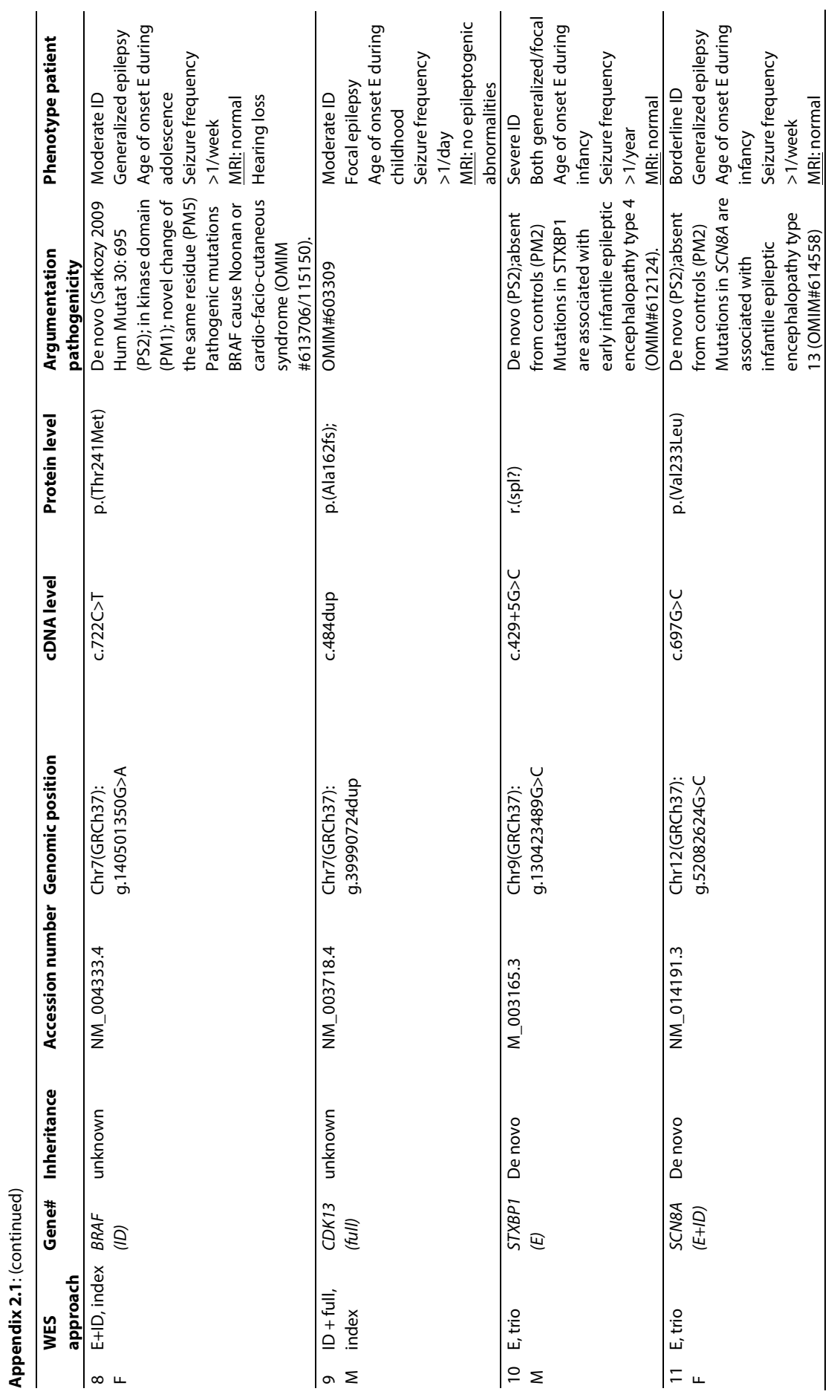




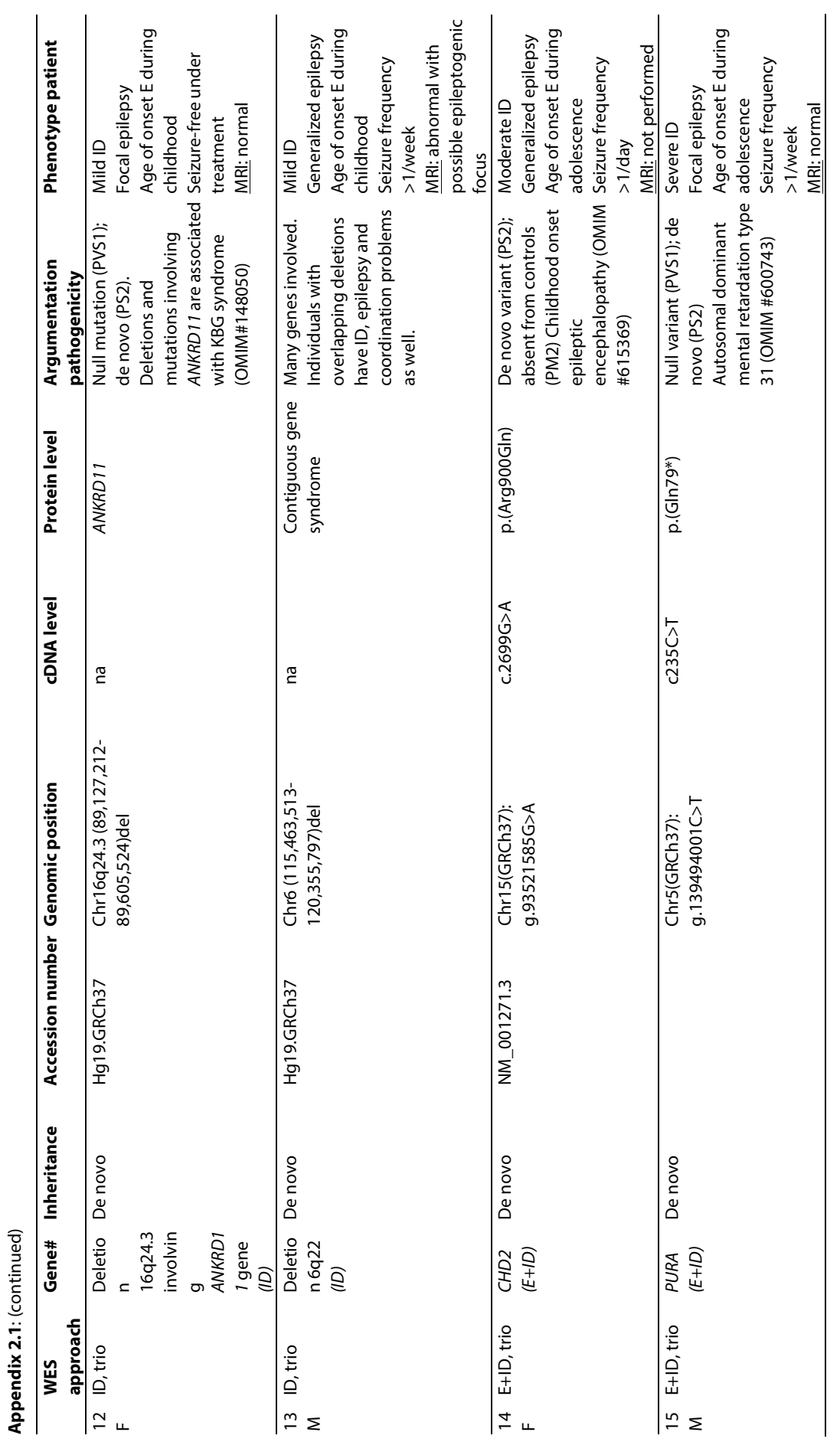




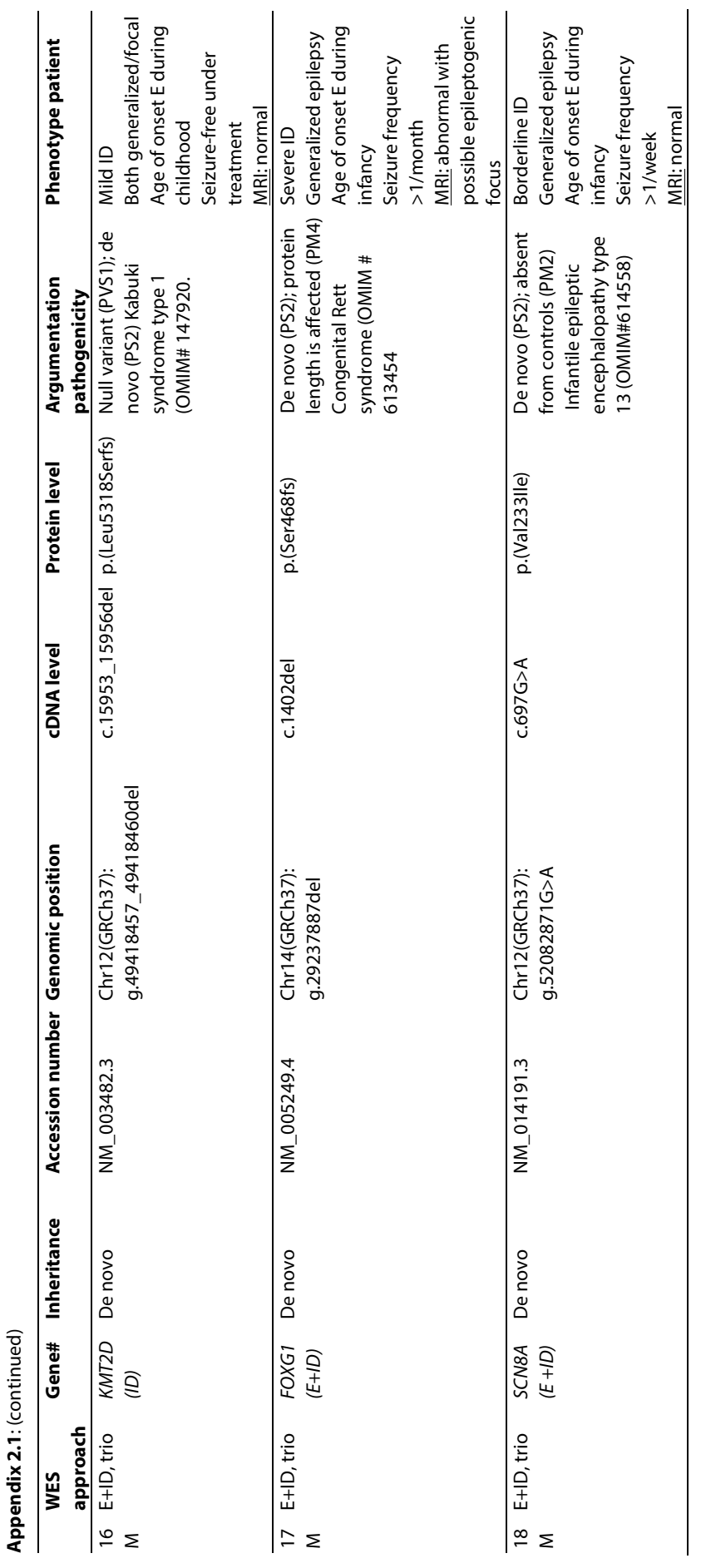




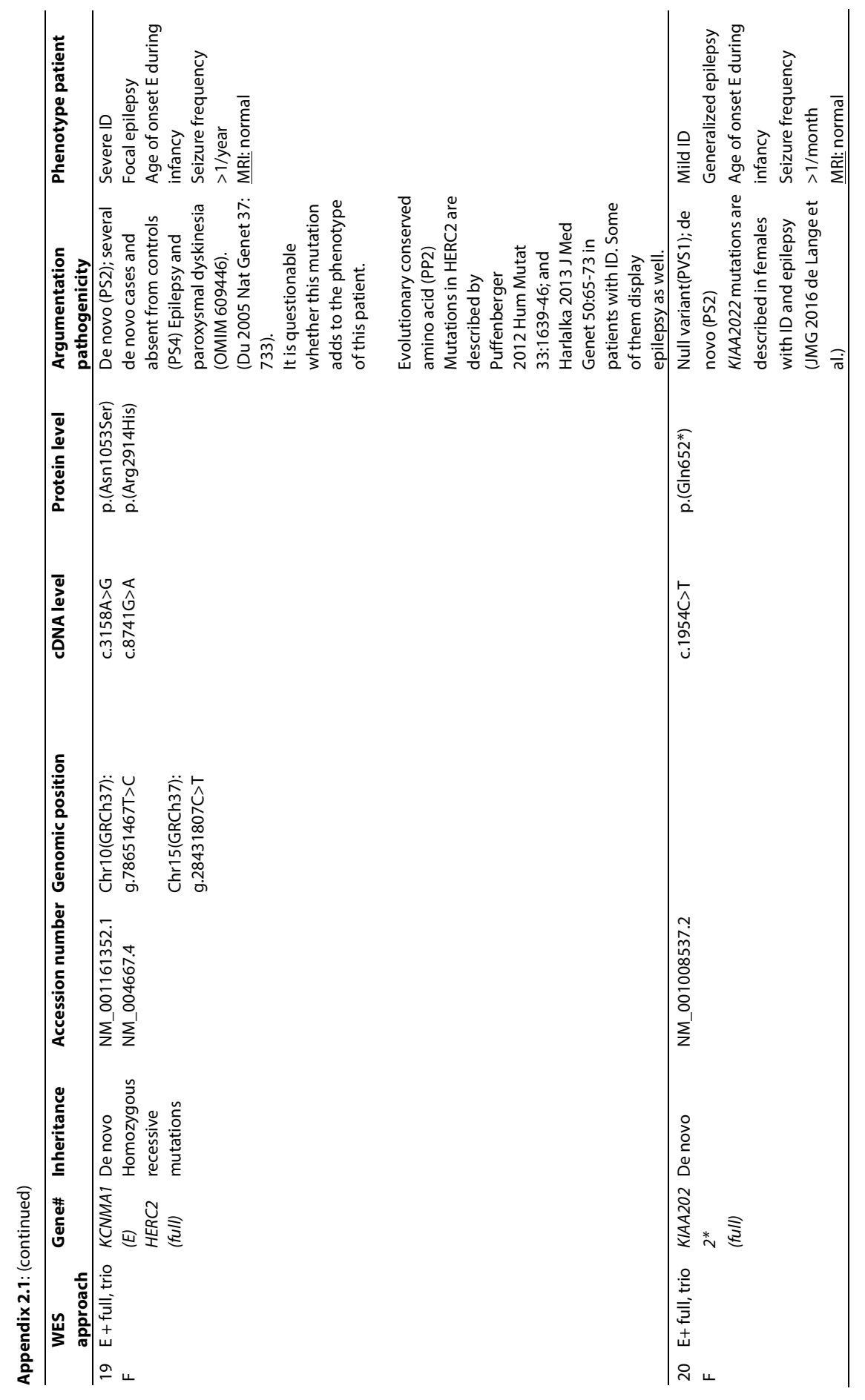




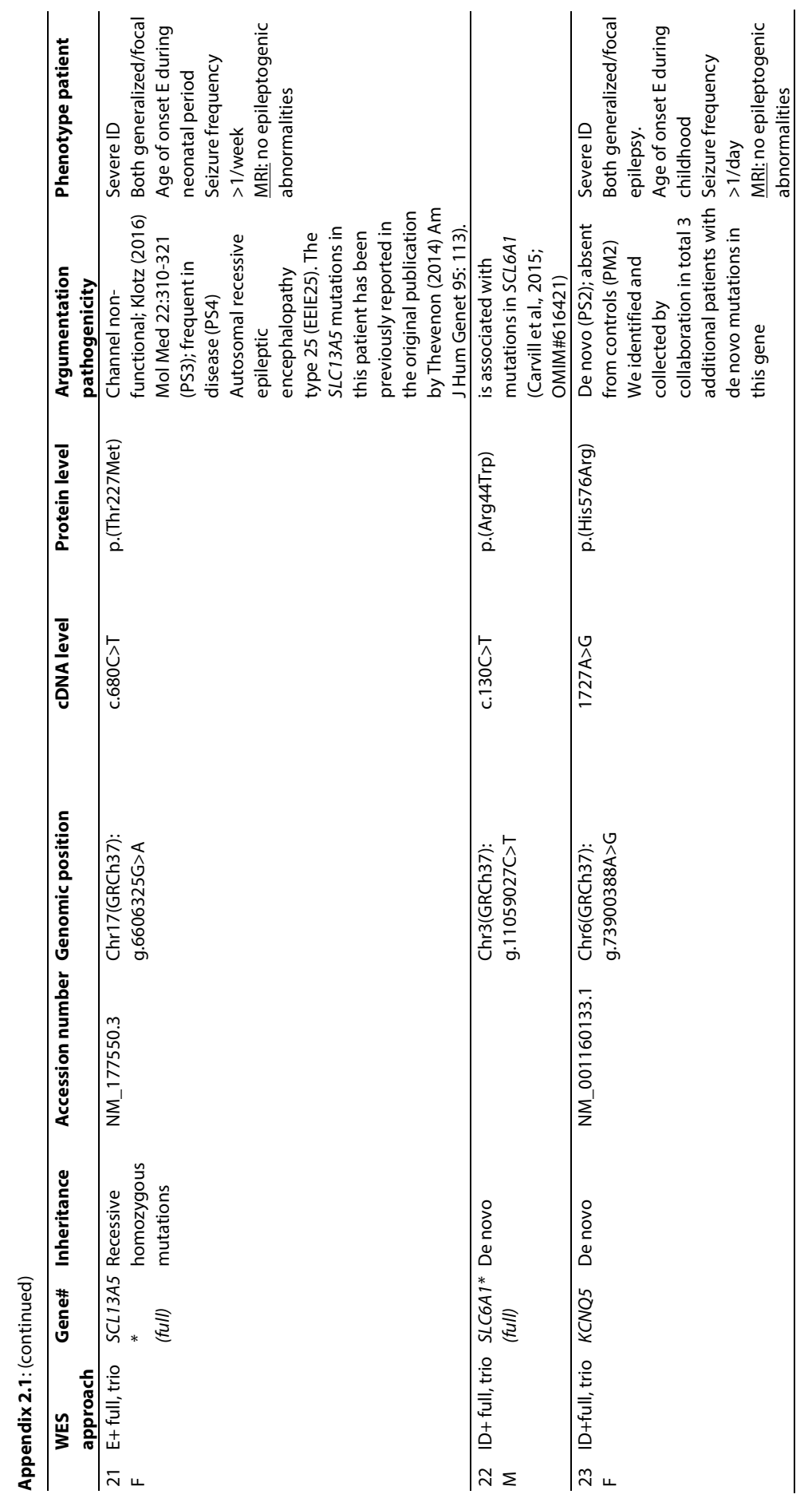




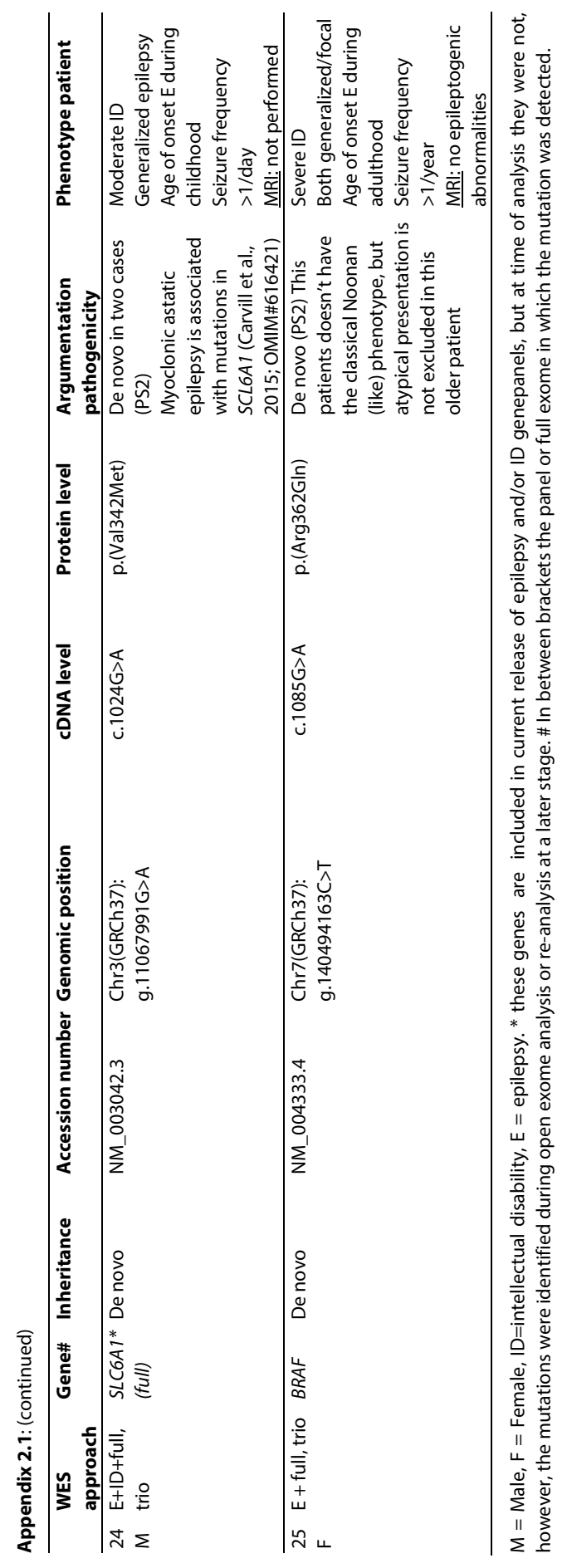




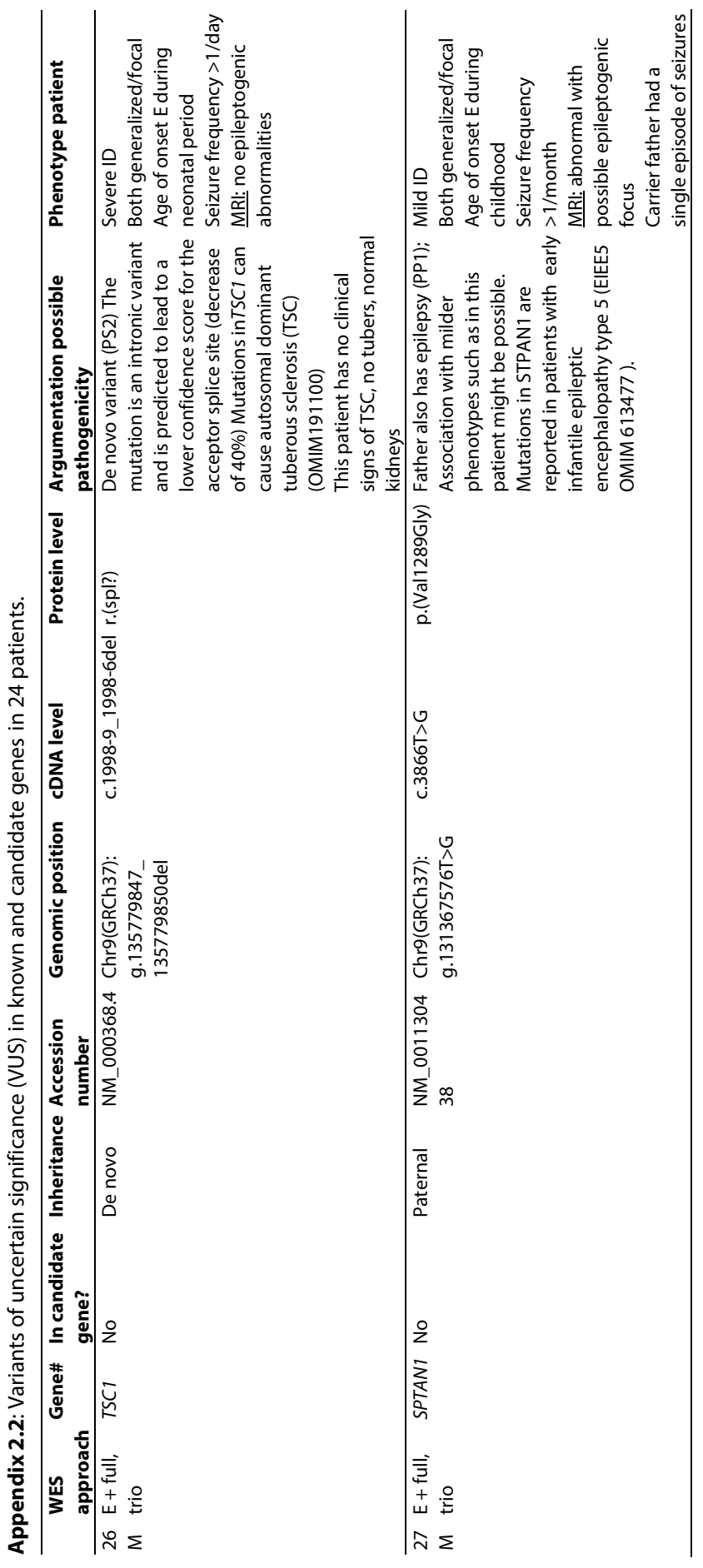




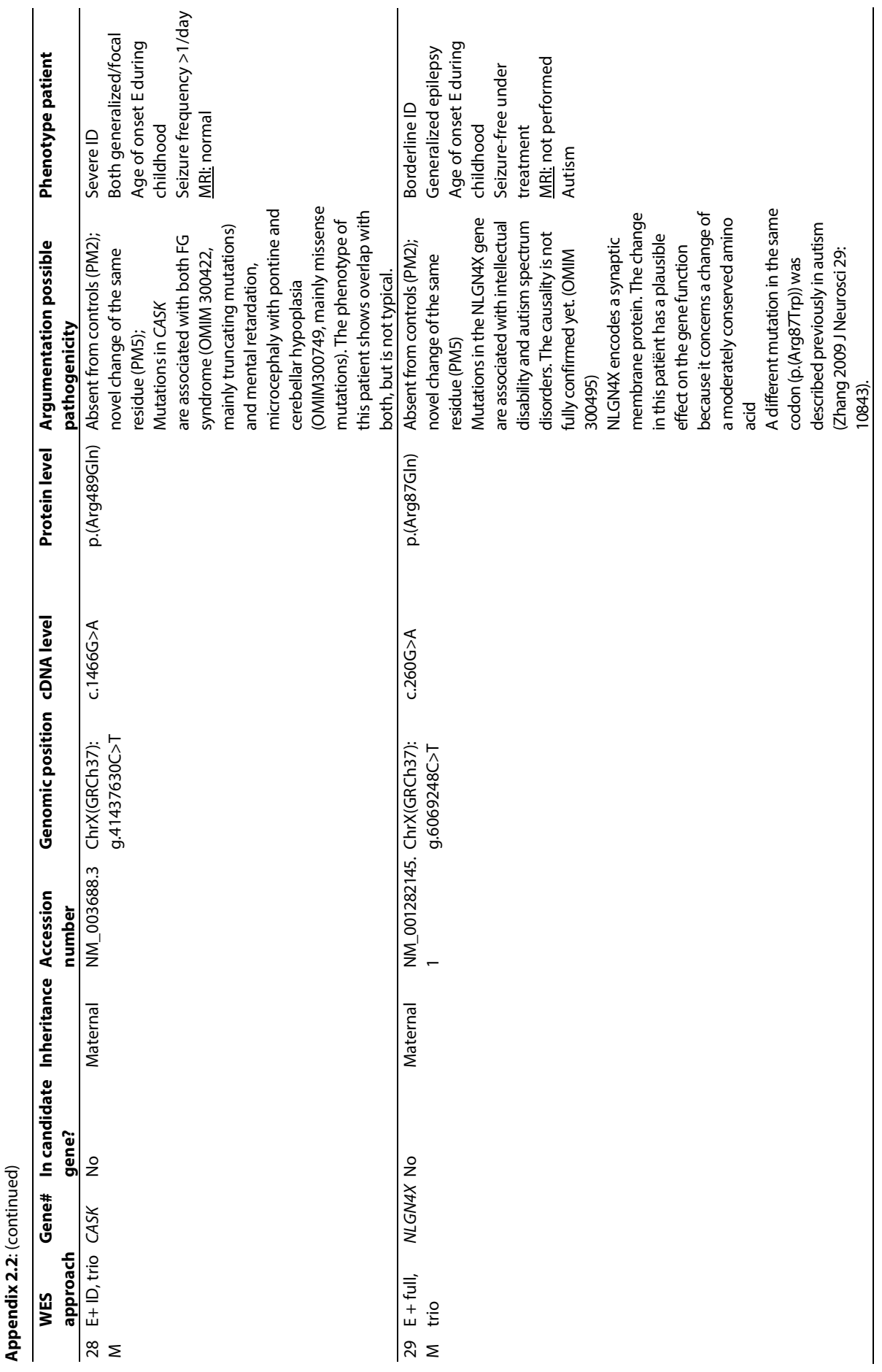




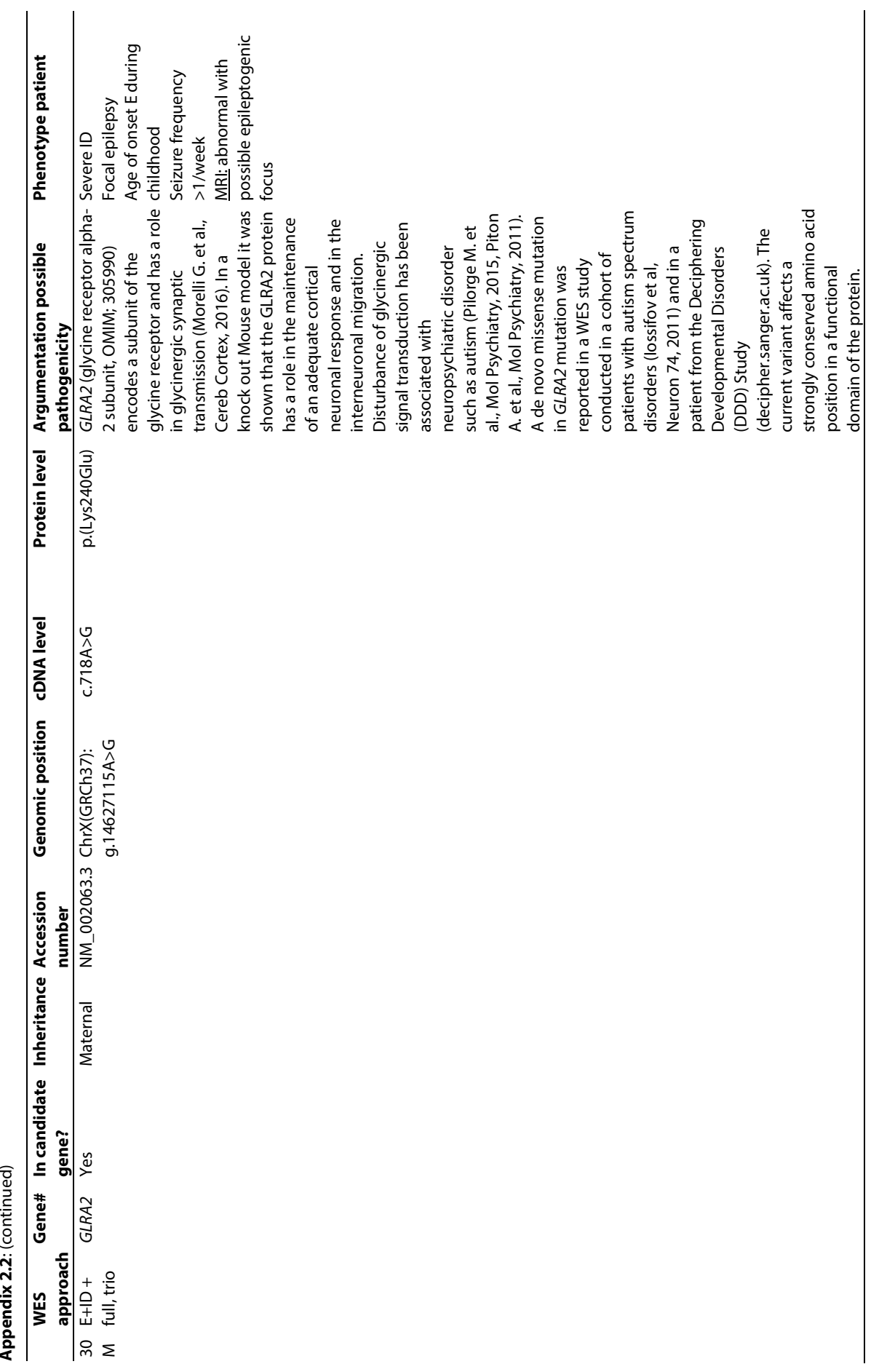




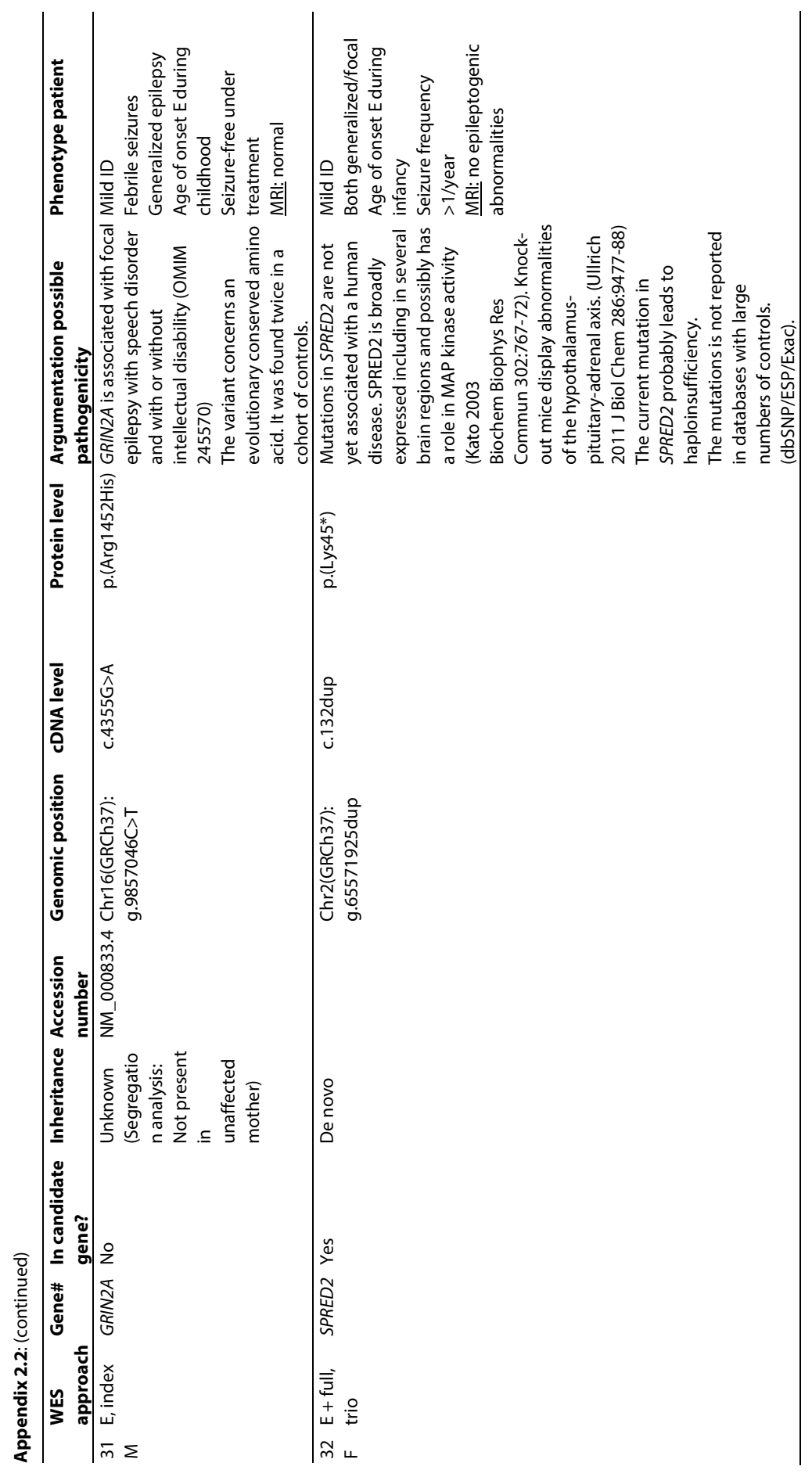




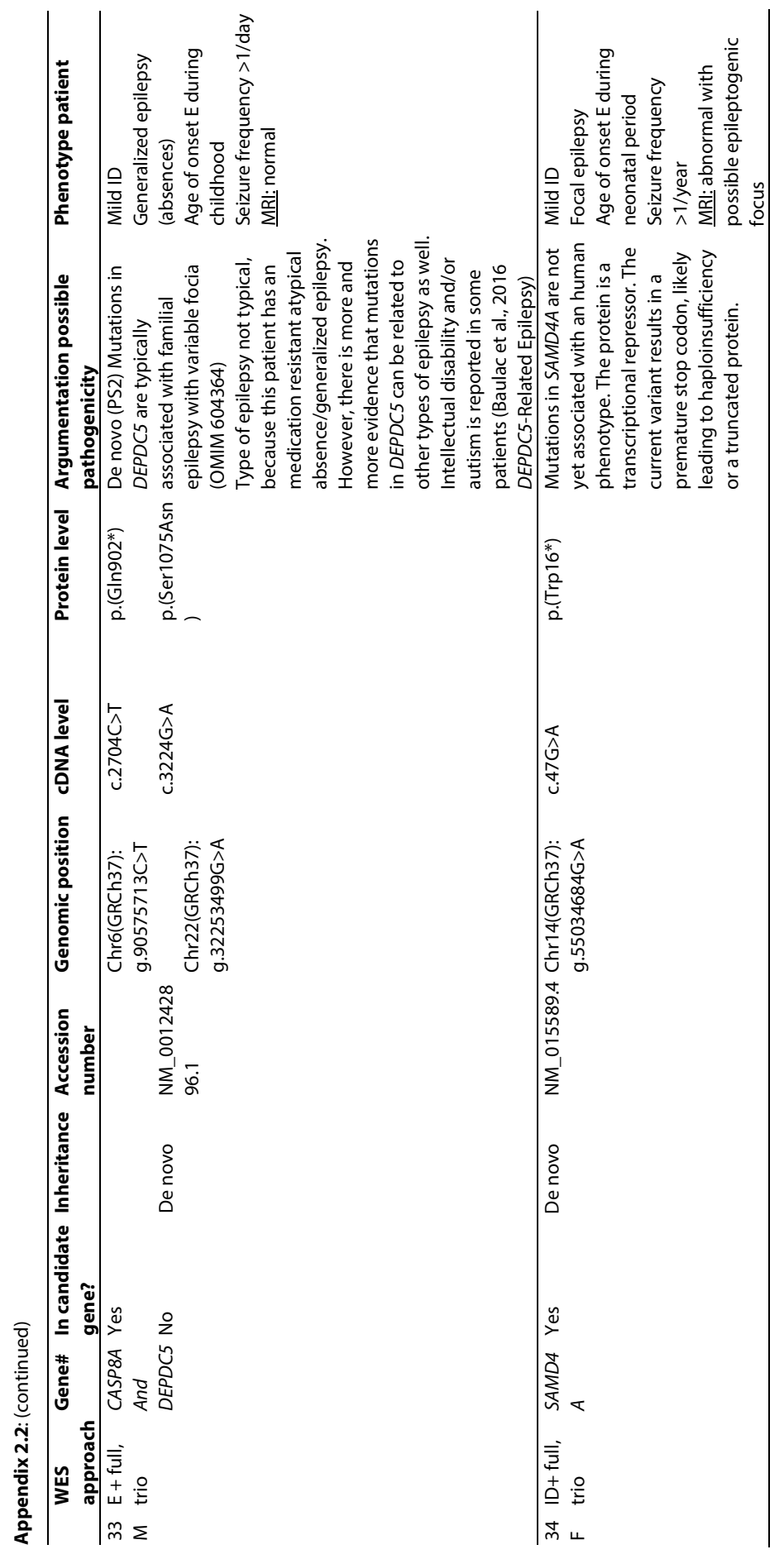




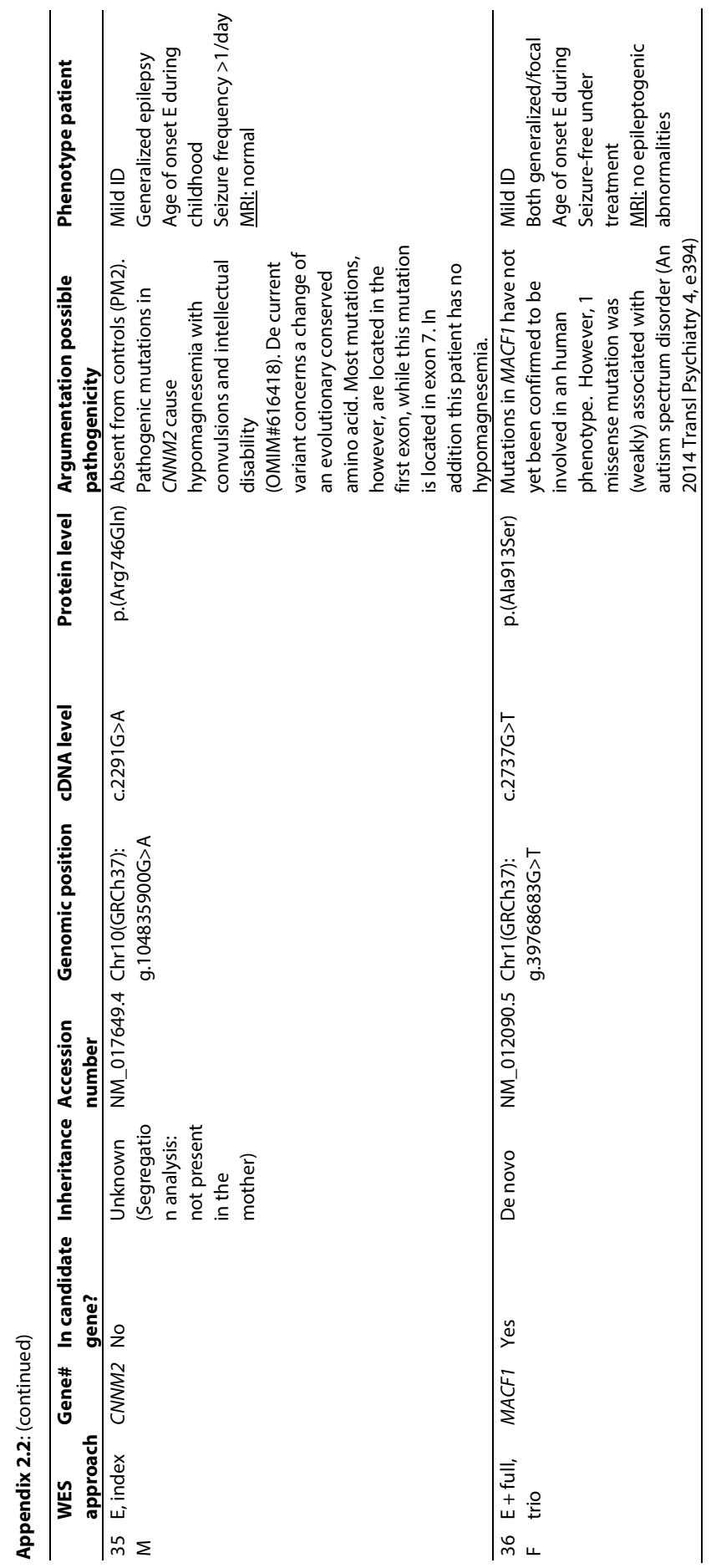




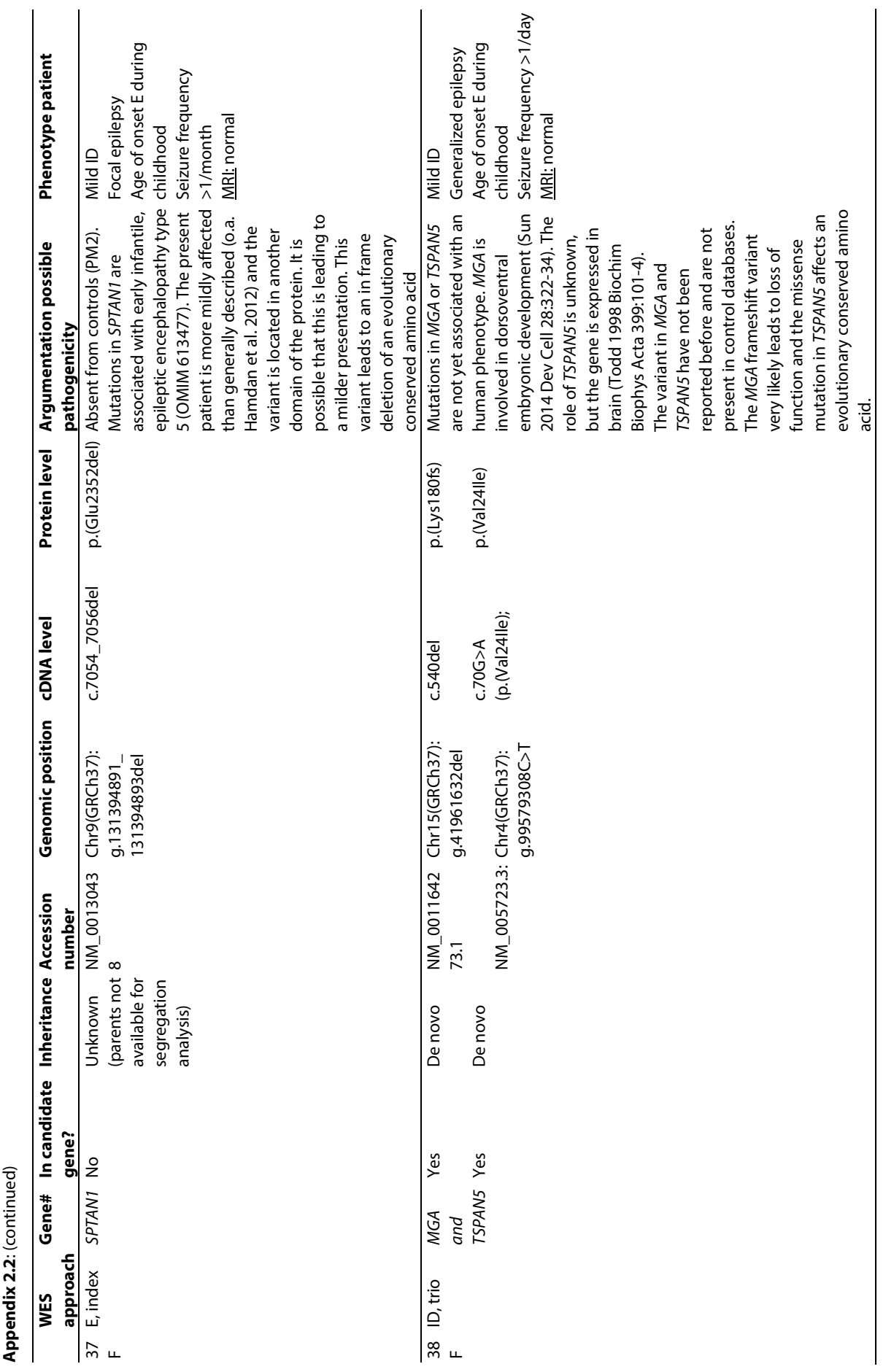




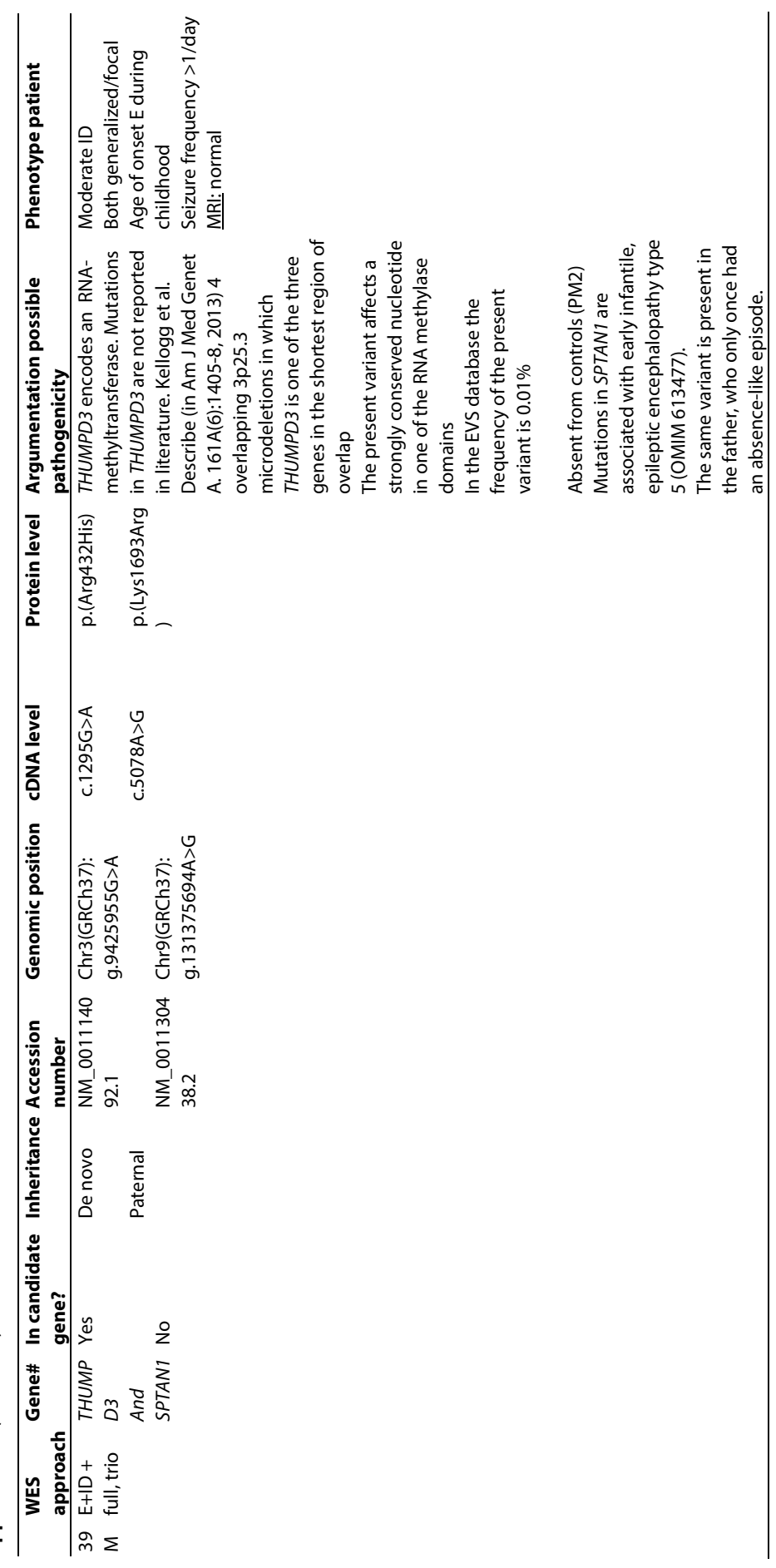




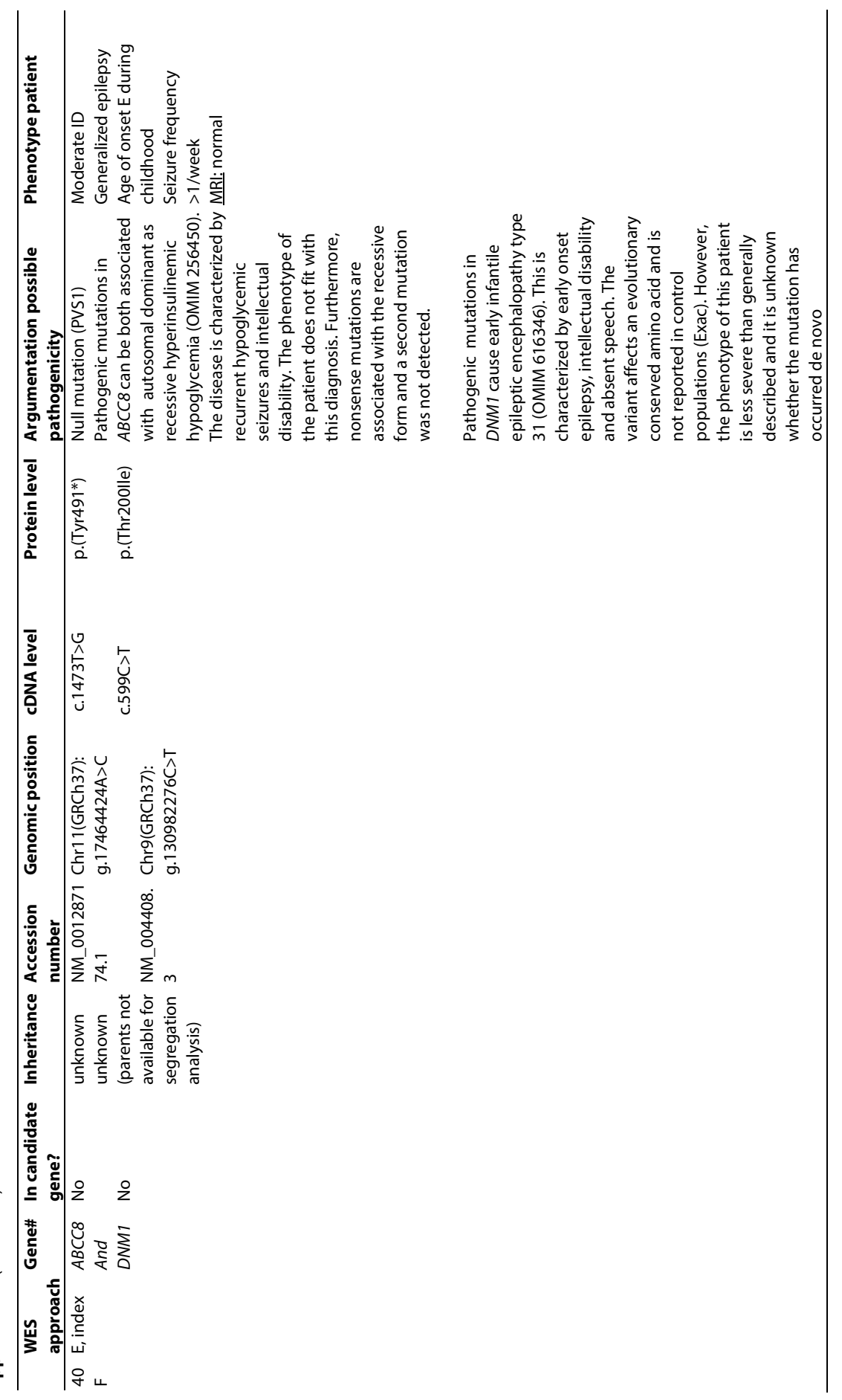




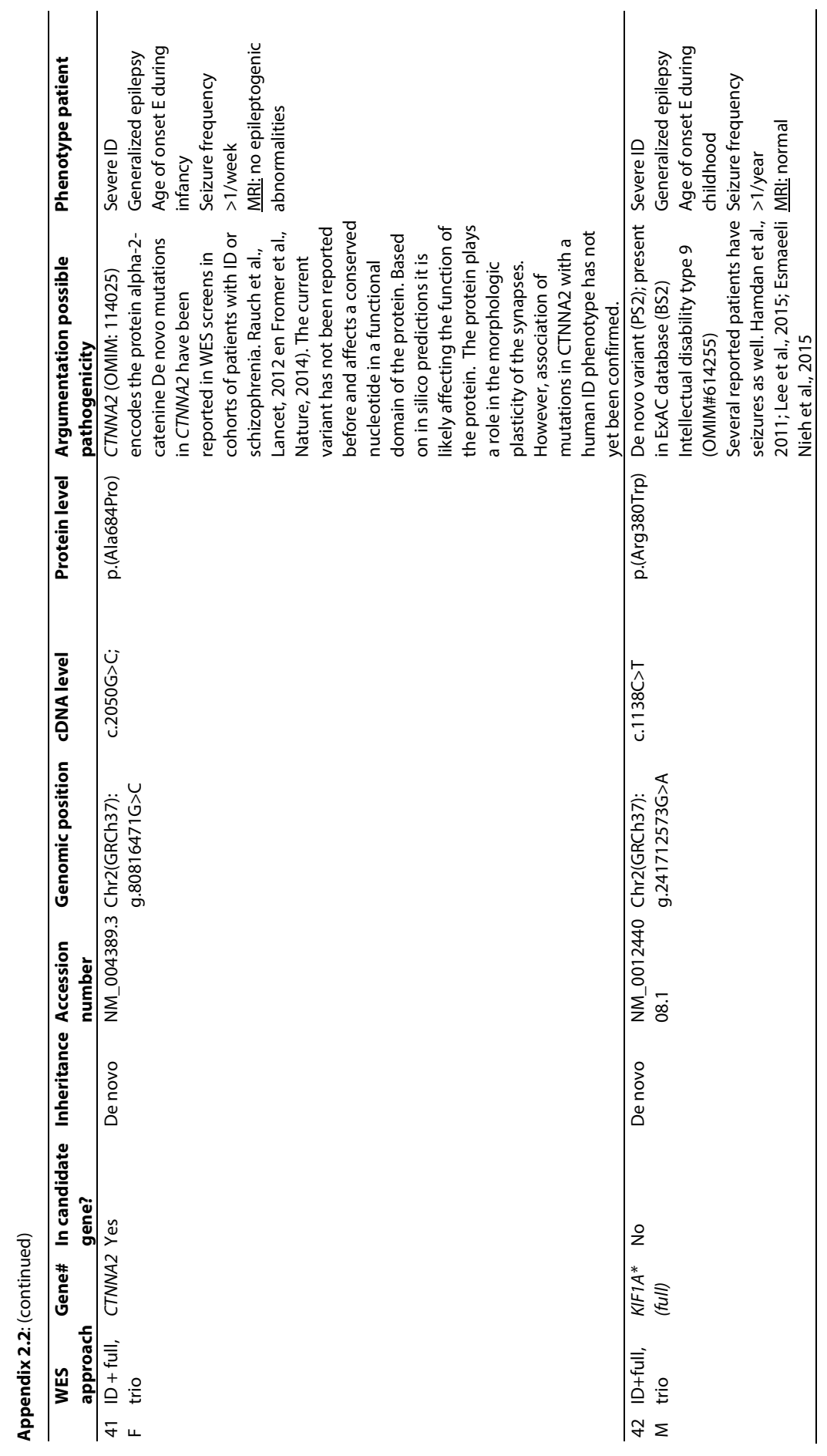




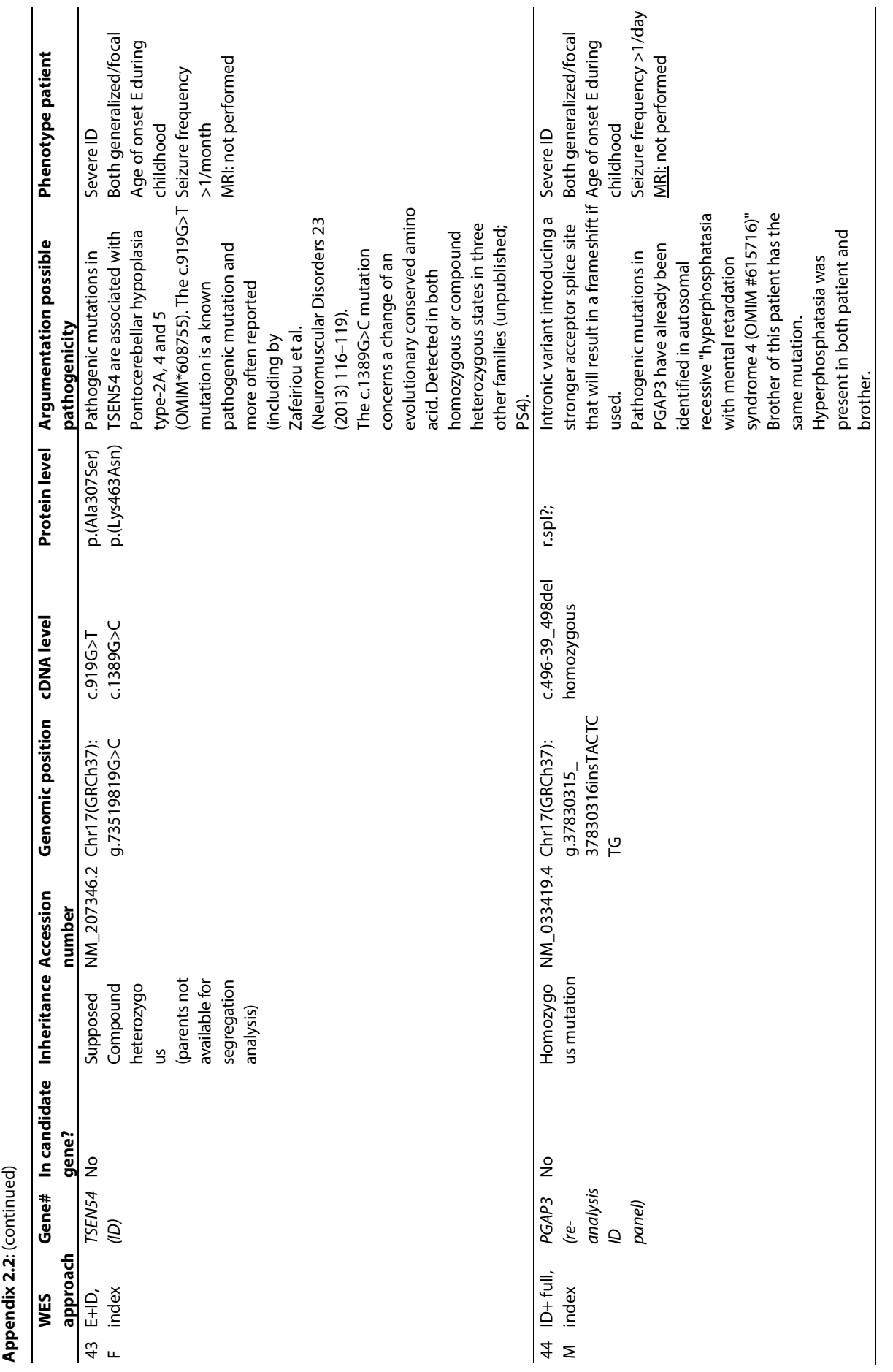




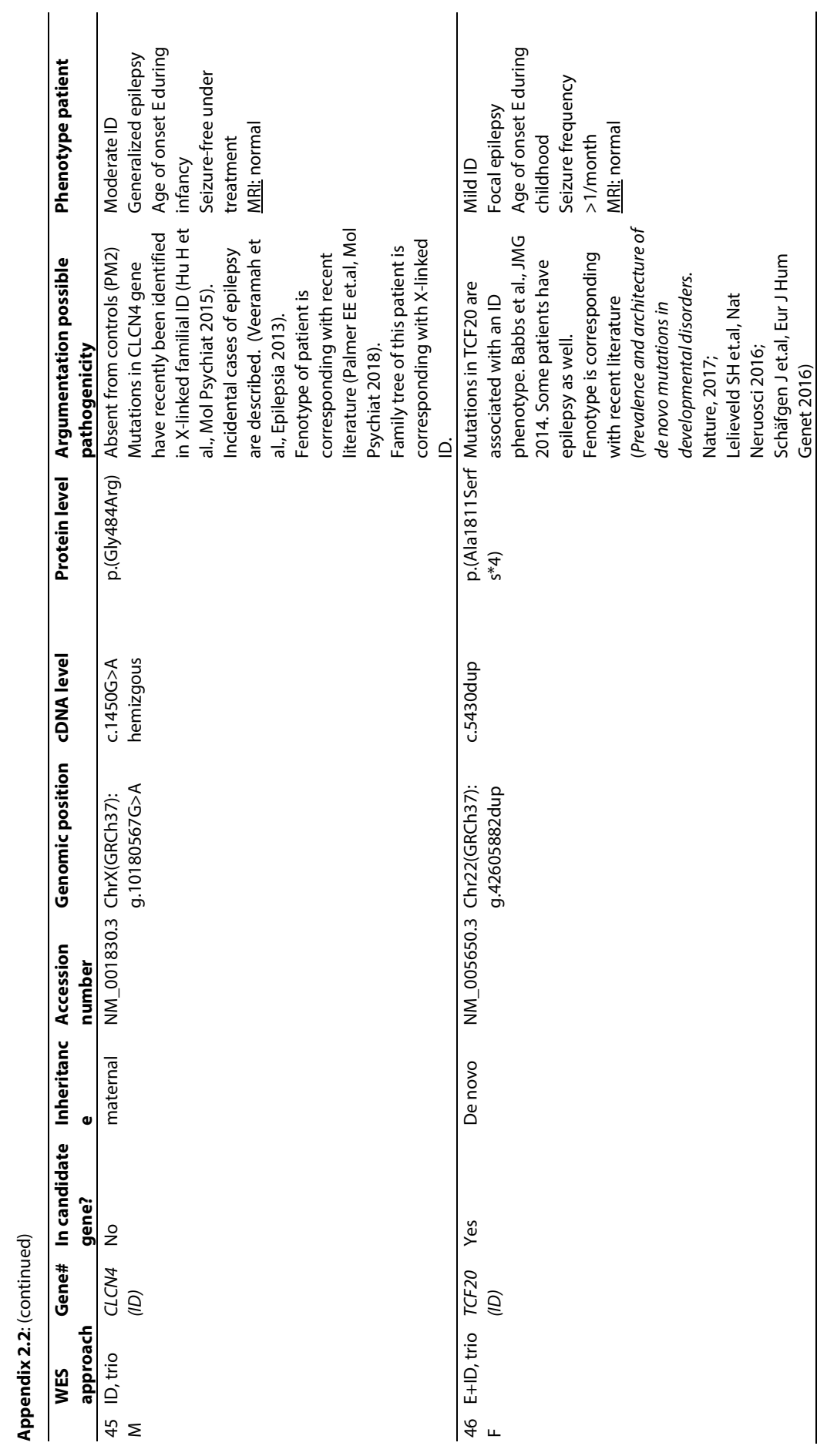




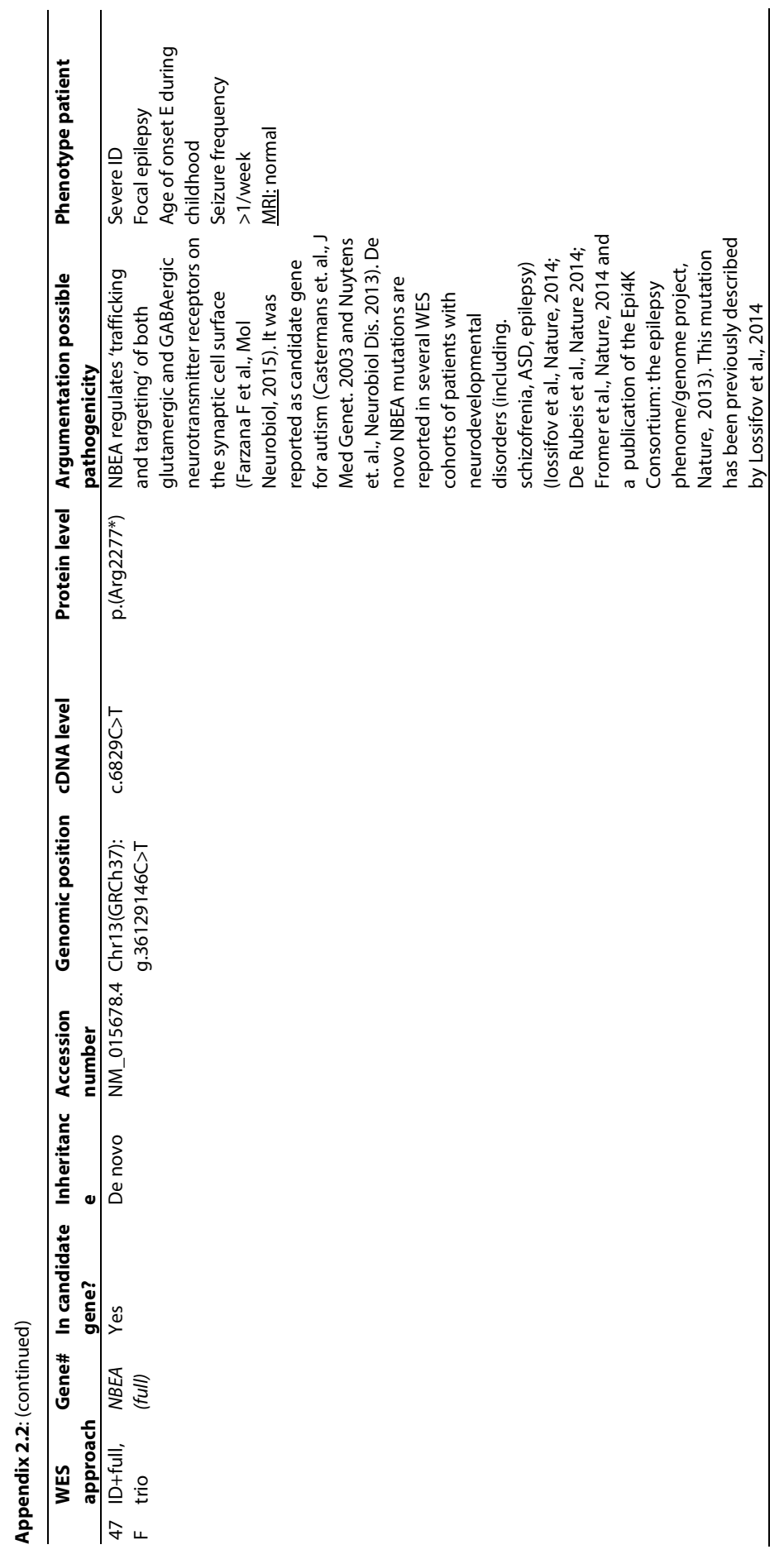


Exome sequencing in epilepsy and ID

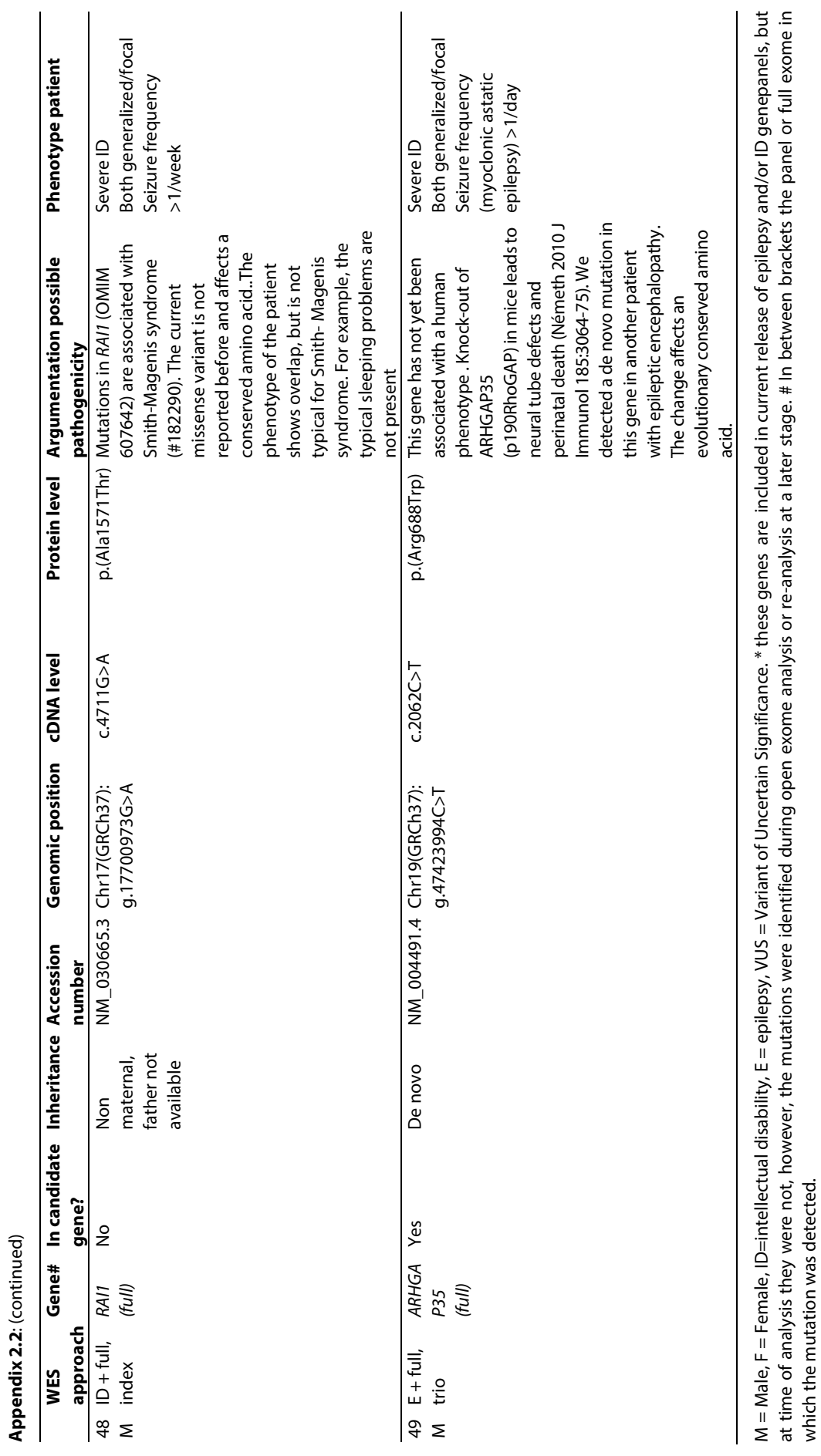




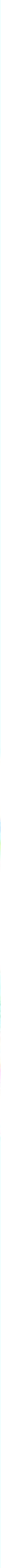




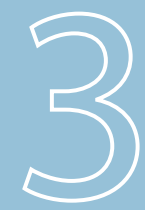

O

\section{Mood, anxiety, and perceived quality of life in adults with epilepsy and ID}

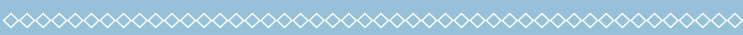

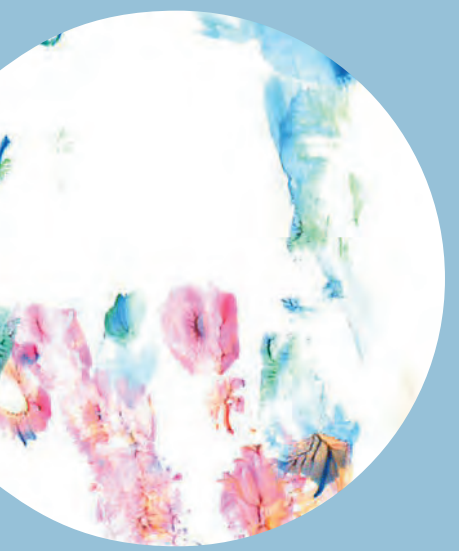

F.M. Snoeijen-Schouwenaars, J.S. van Ool, I.Y. Tan, A.P. Aldenkamp, H.J. Schelhaas, J.G.M. Hendriksen Acta Neurol Scand. 2019;139(6):519-525 


\section{Abstract}

\section{Objective}

Depression and anxiety symptoms are common among patients with epilepsy, but are relatively under-researched in patients with both epilepsy and intellectual disability (ID). The aim was to investigate whether epilepsy and ID characteristics are associated with mood, anxiety and quality of life.

\section{Materials and methods}

Adult patients with epilepsy and ID who rely on tertiary epilepsy care were included $(\mathrm{N}=189)$. Mood, anxiety, and quality of life were assessed by standardized questionnaires. Epilepsy and ID characteristics were retrieved from patient charts or determined by psychometric instruments.

\section{Results}

Elevated levels of depressive and anxiety symptoms were present in $21.7 \%$ and $12.7 \%$, respectively. Anxiety was significantly associated with a focal epilepsy type and ID domain discrepancy (substantial difference between two domains of adaptive behavior), but was negatively related to seizure frequency and drug load of moodstabilizing antiepileptic drugs. Depressive symptoms were not significantly related to epilepsy characteristics, but a severe ID and ID domain discrepancy was associated with more depressive symptoms. Quality of life was significantly worse in those with multiple seizure types and ID domain discrepancy.

\section{Conclusion}

Whereas anxiety and quality of life are associated with individual epilepsy characteristics, this could not be confirmed for depressive symptoms in patients with epilepsy and ID, despite its high prevalence. 


\section{Introduction}

Both depression and anxiety disorders are relatively common psychiatric disorders in patients with epilepsy. Pooled prevalence rates in this population are estimated at $22.9 \%$ and $20.2 \%,{ }^{1}$ which is much higher when compared to the prevalence of depression and anxiety disorders worldwide (4.4\% and 3.6\%). ${ }^{2}$ Although epilepsy relatively often co-occurs with intellectual disability (ID), ${ }^{3}$ literature on the presence of mood disorders among patients with both epilepsy and ID is scarce. Knowledge of this relationship is important as it might influence quality of life in these patients.

Depressive and anxiety symptoms can be associated with epilepsy for multiple reasons. They may have the same underlying neurobiological aetiology ${ }^{4}$ or result from epilepsy due to seizure-related or psychosocial factors, such as increased dependence, experienced stigma and poor seizure control. ${ }^{5,6}$ Results from a systematic review indicate that having an epilepsy diagnosis is associated with an increase of depressive symptoms in adults with ID, and that a severe form of epilepsy might be a risk factor for psychiatric disorders. ${ }^{7}$ More specifically, Espie et al. concluded that psychiatric symptoms were most strongly related to epilepsy characteristics, such as seizure frequency and severity. ${ }^{8}$

Both depressive and anxiety symptoms can have a negative influence on daily functioning and quality of life in this population that is already known for their complex needs. ${ }^{9}$ For example, in a study among 142 adults with epilepsy and mild ID, it was found that psychological distress and seizure frequency were predictors of (health-related) quality of life. ${ }^{10}$

The primary aim of the present study is to investigate whether epilepsy and ID characteristics are associated with depressive symptoms and anxiety in adults with both epilepsy and ID. Our secondary aim is to describe associations between epilepsy and quality of life in a subset of adults with mild ID.

\section{Materials and methods}

\subsection{Study design and participants}

This study had a cross-sectional design and was part of the TRIANGLE study (The Relation between epilepsy, ID, And Neuropsychiatric comorbidities in a Group of patients in Long-term care for Epilepsy), which was conducted amongst in- and 
outpatients who rely on tertiary epilepsy care facilities of Kempenhaeghe, The Netherlands. TRIANGLE is approved by the medical-ethical committee of Kempenhaeghe (No. 15.01) and the medical-ethical committee of Erasmus University medical center concluded that the rules laid down in the Medical Research Involving Human Subjects Act do not apply to this study (MEC-2016-408).

Inclusion criteria were: 1) age $\geq 18$ years, 2) diagnosis of epilepsy according to the clinical definition by the ILAE, ${ }^{11}$ and 3) diagnosis of ID according to DSM-5 ${ }^{12}$ or current adaptive functioning at level of ID as evaluated by the individual's psychologist.

\subsection{Instruments and procedure}

\subsubsection{Mood and anxiety}

Mood and anxiety were assessed using the Dutch version of the Anxiety, Depression and Mood Scale (ADAMS), ${ }^{13,14}$ a by proxy observational questionnaire that is specifically developed for people with ID. The ADAMS consists of 28 items which had to be rated by one professional caregiver on a 4-point scale, ranging from never/no problem to often/severe problem. The Dutch translation of the ADAMS consists of a four-factor structure: Depressive mood, Anxiety, Social avoidance, and Other problems. The validity and reliability of this structure was investigated in two Dutch samples, one sample older than 50 years of age $(\mathrm{N}=198)$ and one adult sample below 50 years of age $(\mathrm{N}=975)^{13,15}$ and was found to be fair to good. Higher scores on (sub)scales are indicative of more anxiety or negative mood. The subscale 'Other problems' and 'social avoidance' were excluded from the analyses, because of the scope of the article and the substantive variability within the subscale.

In addition, information regarding the daily use of psychotropic medication and the history of psychiatric disorders were retrieved from the medical records.

\subsubsection{Quality of life}

The self-reported quality of life was assessed by using the Intellectual Disability Quality of Life questionnaire (IDQOL-16). ${ }^{16}$ This is a 16 -item self-report questionnaire developed for people with a mild ID. Each (written) item is illustrated by pictograms and smileys in order to further clarify the question and response categories, which are based on a 5-point Likert scale varying from very unpleasant to very pleasant. The items correspond to three domains of quality of life: psychological function, social functioning, and satisfaction about daily living. The IDQOL-16 was only administered among subjects with a mild level of ID, if administration of the test was judged feasible by the subject's psychologist (i.e., if the subject was likely to have sufficient 
verbal comprehension). In previous studies, the IDQOL-16 domains were found to have fair to good internal consistency. ${ }^{17,18}$

\subsubsection{Epilepsy characteristics}

Epilepsy characteristics were retrieved from the subject's medical records and included the age at onset, epilepsy type, number of seizure types, number of seizures in the past year, the use and types of anti-epileptic drugs, and the etiology of epilepsy. The diagnosis of epilepsy was classified by a specialized neurologist. Seizures were recorded by the nursing staff. Non-epileptic events, such as psychogenic non-epileptic seizures, were excluded. The epilepsy type and etiology was classified according to the most recent classification system by the International League Against Epilepsy (ILAE). ${ }^{19}$

As a measure for drug load of AEDs with mood stabilizing properties, i.e. carbamazepine, valproic acid, and lamotrigine, the prescribed daily dose versus defined daily dose (PDD/DDD) ratio was calculated. ${ }^{20}$ This was also calculated for benzodiazepines that were prescribed as AED, i.e., clobazam, clonazepam, diazepam, and dipotassium clorazepate. The DDDs were retrieved from the database of the WHO Collaborating Centre for Drug Statistics Methodology. ${ }^{21}$

\subsubsection{Intellectual Disability}

Regarding the ID, we examined the overall level of ID and the presence of an ID domain discrepancy. The level of ID was based on the three domains of adaptive deficits as described in DSM-5: the conceptual, social, and practical domain. ${ }^{22}$ Each domain was assessed separately using standardized instruments and the results were converted into a classification of mild, moderate, severe or profound deficits. An ID profile was considered as discrepant when there was a substantial intra-individual difference between two DSM-5 domains, indicating that one domain is considerably more or less deficient than the other two. For more information regarding the assessment of level of ID and domain discrepancy, see Van Ool et al. ${ }^{23}$

\subsection{Analyses}

Descriptive statistics were calculated for the subscales of the ADAMS as well as Pearson correlation coefficients between subscales. Also, the most reported items and the number of subjects having a score above the clinical cut-off for depressive symptoms $(\geq 14)$ or anxiety $(\geq 10)$ were calculated. ${ }^{24}$

Separate linear regression analyses were performed to examine direct associations between each epilepsy and ID characteristic and ADAMS subscale scores. 
Subsequently, multiple hierarchical regression analyses were performed in which predictors were added to the model in three steps. In the first step, demographic variables (age and sex) and the use of psychotropic drugs were entered, in the second step, level of ID and the presence of an ID discrepancy was added, and in the final model, the epilepsy characteristics were added. Model statistics as well as predictor statistics were examined. Associations between predictors and quality of life were assessed using Pearson correlation analyses and independent samples ttests (or nonparametric alternatives if the variables do not meet the normality assumption). Results were considered significant if $p<0.05$. All analyses were conducted in IBM SPSS Statistics version 24 .

\section{Results}

\subsection{Sample characteristics}

A total of 240 patients were invited for the study, of whom 189 provided consent for the study (inclusion rate: $78.8 \%$ ). The consent was provided by individuals and/or their legal guardian when appropriate. Participants were significantly younger than the 51 nonparticipants (mean difference $=6.04$ years, $p=0.015$ ) and were using psychotropic medication more often $(41.8 \%$ versus $14.0 \%$ respectively, $p<0.001)$. Individuals who participated did not differ from non-participants with respect to level of ID or gender. Twenty-four subjects (12.7\%) with a mild ID had completed the quality of life questionnaire.

The sample comprised $58.7 \%$ males and had a mean age of 47.9 years $(S D=15.6$; range $18.3-85.9$ years). The majority of subjects resided in residential facilities (76.2\%); the others lived in community settings. The level of ID was mild in $20.1 \%$, moderate in $30.7 \%$, severe in $29.1 \%$, and profound in $20.1 \%$, and an ID domain discrepancy was present in $32.8 \%$. Clinical characteristics are described in Table 3.1. The descriptive statistics of the ADAMS subscales are presented in Table 3.2. Elevated levels of depressive and anxiety symptoms were present in $21.7 \%$ and $12.7 \%$, respectively. The subscales were moderately to highly correlated (Pearson $r$ varying from 0.426 to 0.571 , all $P$-values <0.001). The ADAMS items that were reported most frequently, were: tense $(74.1 \%)$, does not relax $(70.9 \%)$, fatigued $(64.6 \%)$, distracted $(63.4 \%)$, and lacked energy (63.4\%).

Reliability analyses on the ADAMS subscales showed fair to good internal consistency for the study population (Cronbach's $a=0.795$ to 0.850 ). Regarding the IDQOL, the psychological domain had a good internal consistency $(a=0.83)$. The 
domains social functioning and satisfaction about daily living had, however, insufficient internal consistency ( $a=0.50$ and $a=0.58$, respectively), and were therefore excluded from the analyses.

Table 3.1: Clinical characteristics of the study sample $(\mathrm{N}=189)$.

\begin{tabular}{|c|c|}
\hline Characteristics & Values \\
\hline Age at onset of epilepsy (years) & $M d n=2.0, I Q R=0-5.5$, range $0-53$ \\
\hline Infancy (< 1 yr) & $32.8 \%$ \\
\hline Childhood (1 - $12 \mathrm{yr})$ & $54.0 \%$ \\
\hline Adolescence (12 - $18 \mathrm{yr})$ & $10.1 \%$. \\
\hline Adulthood (18+yr) & $3.2 \%$ \\
\hline \multicolumn{2}{|l|}{ Epilepsy type $\mathrm{a}^{\mathrm{a}}$} \\
\hline Generalized only & $10.6 \%$ \\
\hline Focal only & $41.3 \%$ \\
\hline Both generalized and focal & $44.4 \%$ \\
\hline Missing/unknown & $3.7 \%$ \\
\hline Number of seizure types (semiology) ${ }^{a}$ & $M d n=3.0, I Q R=1-4$, range $0-8$ \\
\hline Seizure frequency (last year) & $M d n=70.0, I Q R=11.5-153.0$, range $0-1206$ \\
\hline Seizure-free & $12.7 \%$ \\
\hline Yearly & $12.2 \%$ \\
\hline Monthly & $19.6 \%$ \\
\hline Weekly & $43.9 \%$ \\
\hline Daily & $11.6 \%$ \\
\hline \multicolumn{2}{|l|}{ Etiology of epilepsy ${ }^{a}$} \\
\hline Structural & $28.6 \%$ \\
\hline Genetic & $20.1 \%$ \\
\hline Infectious & $6.3 \%$ \\
\hline Metabolic & $1.1 \%$ \\
\hline Unknown & $43.9 \%$ \\
\hline Daily use of anti-epileptic drugs & $M d n=3.0, I Q R=2.5-4.0$, range $0-6$ \\
\hline Daily use of psychotropic drugs & $41.8 \%$ \\
\hline Psychiatric classification (DSM-IV) & $20.6 \%$ \\
\hline
\end{tabular}

a Based on ILAE 2017 criteria.19 ID = intellectual disability.

Table 3.2: Descriptive statistics of the ADAMS subscales $(\mathrm{N}=189)$

\begin{tabular}{lcccc}
\hline & M & SD & Range & Above clinical cut-off $^{\mathbf{a}}$ \\
\hline Depressive symptoms & 9.99 & 7.04 & $0-34$ & $21.7 \%$ \\
Anxiety & 4.73 & 3.73 & $0-15$ & $12.7 \%$ \\
\hline
\end{tabular}

${ }^{a}$ Based on cut-off values provided by Hermans et al. ${ }^{24}$

\subsection{Associations between epilepsy, ID, and mood and anxiety}

Results of the linear regression analyses predicting depressive symptoms, anxiety, and social avoidance are presented in Table 3.3. The models yielded different results per outcome measure. For both depressive symptoms and anxiety, adding ID 
characteristics (severity of ID and presence of ID domain discrepancy) resulted in a significant increase in explained variance $(R 2$ change $=0.053, p=0.004$ and $R 2$ change $=$ $0.033, p=0.026$, respectively). The introduction of epilepsy characteristics (i.e., number of seizure types, seizure frequency, epilepsy type, drug load of mood stabilizing AED and of benzodiazepine AED, and use of rescue medication) in the final model did not yield a significant increase in explained variance of depressive symptoms, but did for anxiety ( $R 2$ change $=0.034, \mathrm{p}=0.301$ and $R 2$ change $=0.087, \mathrm{p}=0.002$, respectively). Hence, the final models explained $20.0 \%$ of the variance in depressive symptoms $(F=3.86, p<0.001)$ and $31.0 \%$ of the variance in anxiety scores $(F=6.95, p<0.001)$.

Table 3.3: Linear regression analyses predicting affective outcomes.

\begin{tabular}{|c|c|c|c|c|c|c|}
\hline \multirow[t]{2}{*}{ Unadjusted analyses } & \multirow[b]{2}{*}{ B (SE) } & \multirow[b]{2}{*}{$\boldsymbol{\beta}$} & \multicolumn{4}{|c|}{ Adjusted analyses $^{a}$} \\
\hline & & & $\mathbf{p}$ & B (SE) & $\boldsymbol{\beta}$ & $\mathbf{p}$ \\
\hline \multicolumn{7}{|l|}{ Depressive symptoms } \\
\hline Number of seizure types & $0.14(0.27)$ & 0.04 & 0.596 & $-0.42(0.38)$ & -0.11 & 0.270 \\
\hline Seizure frequency & $0.19(0.03)$ & 0.05 & 0.531 & $0.03(0.04)$ & 0.08 & 0.884 \\
\hline Focal epilepsy & $0.22(1.67)$ & 0.10 & 0.198 & $2.23(1.58)$ & -0.10 & 0.160 \\
\hline Drug load mood-stabilizing & $-0.91(0.71)$ & -0.09 & 0.205 & $-0.59(0.70)$ & -0.06 & 0.399 \\
\hline \multicolumn{7}{|l|}{ AED } \\
\hline Drug load benzodiazepine AED & $0.42(0.81)$ & 0.04 & 0.607 & $0.44(0.80)$ & 0.04 & 0.552 \\
\hline Use of rescue medication & $-0.05(0.05)$ & -0.06 & 0.379 & $-0.08(0.06)$ & -0.10 & 0.183 \\
\hline ID discrepancy & $2.79(1.07)$ & 0.19 & $0.010^{*}$ & $3.25(1.13)$ & 0.22 & $0.004^{* *}$ \\
\hline Level ID (severe/profound) & $0.43(1.03)$ & 0.03 & 0.673 & $3.05(1.22)$ & 0.22 & $0.013^{*}$ \\
\hline \multicolumn{7}{|l|}{ Anxiety } \\
\hline Number of seizure types & $-0.04(0.14)$ & -0.02 & 0.803 & $0.09(0.19)$ & 0.04 & 0.653 \\
\hline Seizure frequency & $-0.03(0.02)$ & -0.15 & $0.035^{*}$ & $-0.05(0.02)$ & -0.23 & $0.006^{* *}$ \\
\hline Focal epilepsy & $1.74(0.88)$ & 0.15 & $0.050^{*}$ & $1.67(0.78)$ & 0.14 & $0.034^{*}$ \\
\hline Drug load mood-stabilizing & $-0.73(0.38)$ & -0.140 & 0.053 & $-0.91(0.35)$ & -0.17 & $0.009^{* *}$ \\
\hline \multicolumn{7}{|l|}{ AED } \\
\hline Drug load benzodiazepine AED & $-0.06(0.43)$ & -0.01 & 0.898 & $0.22(0.40)$ & 0.04 & 0.546 \\
\hline Use of rescue medication & $-0.03(0.03)$ & -0.08 & 0.254 & $-0.01(0.3)$ & -0.02 & 0.788 \\
\hline ID discrepancy & $1.82(0.56)$ & 0.23 & $0.001^{* *}$ & $1.45(0.56)$ & 0.18 & $0.010^{* *}$ \\
\hline Level ID (severe/profound) & $-0.72(0.54)$ & -0.10 & 0.187 & $0.47(0.60)$ & 0.06 & 0.435 \\
\hline
\end{tabular}

Essentially, having a more severe level of ID and the presence of an ID domain discrepancy were significantly associated with more depressive symptoms $(p=0.013$ and $p=0.004$, respectively). Anxiety levels were also significantly associated with the presence of an ID domain discrepancy $(p=0.010)$, but not with the level of ID. Regarding epilepsy, none of the epilepsy characteristics were related to depressive symptoms. Anxiety levels were, however, significantly higher in subjects with focal epilepsy $(p=0.034)$. Lower levels of anxiety were significantly associated with a high 
drug load of mood stabilizing AEDs (carbamazepine, valproic acid, and lamotrigine) and a high seizure frequency $(p=0.009$ and $p=0.006$, respectively). See Figure 3.1 for an overview of statistically significant predictors.

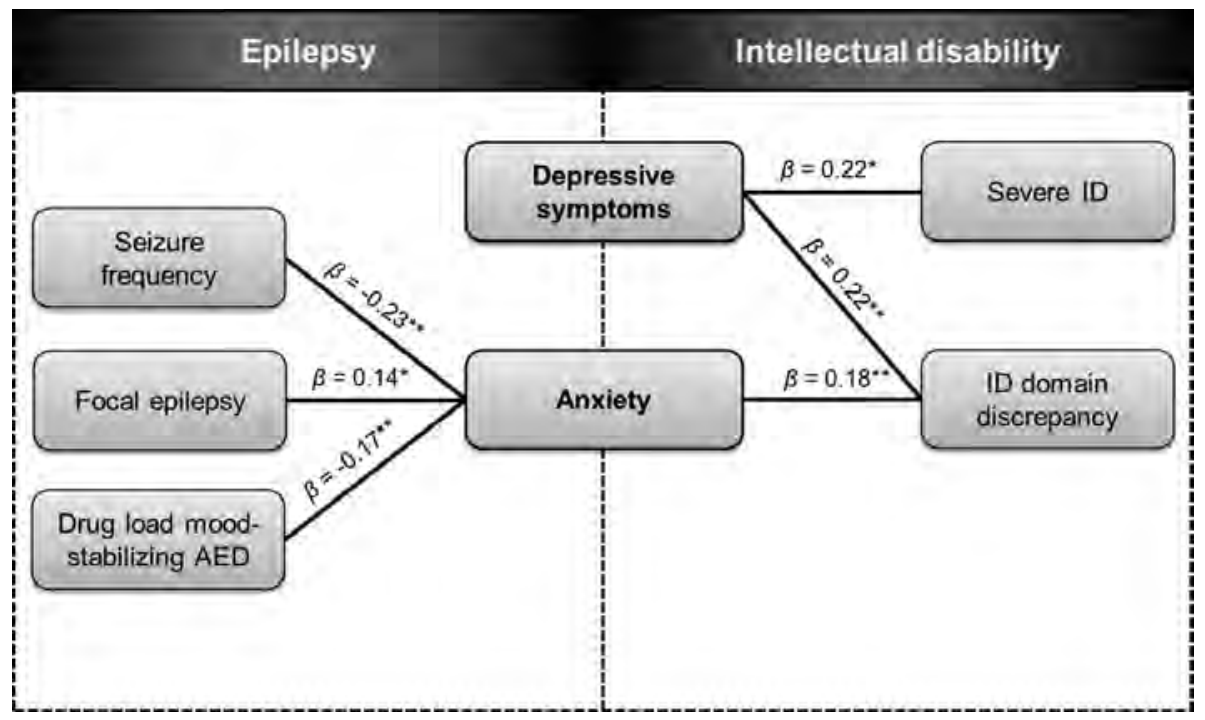

Figure 3.1: Overview of factors significantly associated with depressive symptoms and anxiety.

Post-hoc regression analyses were performed to investigate which ID domains were particularly low in case of high levels of mood and anxiety. Results of these analyses indicated that depressive symptoms were significantly higher when having a discrepancy at the expense of the practical domain $(B=3.42, S E=1.54, p=0.028)$, anxiety levels were mostly linked to a discrepancy at the expense of the social domain $(B=1.18, S E=0.68, p=0.086)$, and there was significantly more social avoidance when having a discrepancy at the expense of the social domain $(B=1.57, S E=0.74, p=0.035)$.

\subsection{Self-reported quality of life}

Spearman Rank correlation analyses were performed to investigate the associations between the IDQOL's subscale psychological functioning and number of seizure types, seizure frequency, and the PDD/DDD ratio of mood stabilizing AEDs (see Table 3.4). There was a strong, negative association between the number of seizure types and the reported quality of life with respect to psychological functioning ( $r h o=-0.551$, $p=0.005$ ). Although not statistically significant, there was a clinically relevant 
association with a medium effect between quality of life and seizure frequency (rho=-0.349, $\mathrm{p}=0.094$ ) and PDD/DDD ratio of mood stabilizing AEDs (rho=0.312, $\mathrm{p}=0.138$ ), with a higher frequency related to a poorer quality of life and a higher drug load of mood stabilizing AEDs related to a better quality of life. In addition, subjects who had an ID domain discrepancy reported a significant poorer quality of life than subjects without a discrepancy (difference in medians $=3.0, p=0.020$ ). There was no association between seizure type and quality of life. Also, correlation analyses between ADAMS subscales and IDQOL psychological functioning yielded no significant associations (Spearman's rho varying from -0.224 to -0.016 , all $p$ values $>0.05$ ).

Table 3.4: Associations between epilepsy characteristics and quality of life ( $\mathrm{N}=24$ patients with mild ID).

\begin{tabular}{lll}
\hline & Quality Of Life - psychological functioning & P \\
\hline Number of seizure types & Spearman $r=-0.551$ & $0.005^{* *}$ \\
Seizure frequency & Spearman $r=-0.349$ & 0.094 \\
PDD/DDD ratio mood AEDs & Spearman $r=0.312$ & 0.138 \\
Both focal and generalized epilepsy & & 0.423 \\
Yes & Mdn $=20.5, \mathrm{IQR}=19.0-24.3$ & \\
No (only focal) & Mdn $=20.0, \mathrm{IQR}=18.0-22.0$ & $0.020^{*}$ \\
ID discrepancy & & \\
Yes & $M d n=22.0, \mathrm{IQR}=19.0-23.0$ & \\
No & $M d n=19.0, I Q R=11.5-20.0$ & \\
\hline
\end{tabular}

\section{Discussion}

Depressive and anxiety symptoms are a clinically relevant and important issue among patients with epilepsy and ID, as $21.7 \%$ and $12.7 \%$ of them show evidence for elevated levels of depressive or anxiety symptoms. This rate of depressive symptoms is in line with the pooled prevalence of depression estimated at $22.9 \%$ for patients with epilepsy in general. ${ }^{1}$ However, the results of our study suggests that the prevalence of anxiety is less pronounced in patients with both epilepsy and ID in our study as compared to the pooled prevalence of anxiety disorders (20.2\%) in the general population of patients with epilepsy. ${ }^{1}$ Besides the findings and hypothesis written below, this might also be due to measurement aspects of our study. For example, we only used the ADAMS, a by-proxy observational questionnaire, which isn't available in the general population.

The associations between epilepsy and ID characteristics in relation to mood and anxiety were different per affective outcome. Epilepsy was significantly associated with anxiety in both positive as well as negative directions. While adjusting for 
demographics and other predictors, higher levels of anxiety were associated with having a focal epilepsy type. Although we did not have information about the localization of the epilepsies in our sample, the link between focal epilepsy and anxiety has also been described in previous studies ${ }^{25,26}$ and might be explained by the involvement of temporal brain structures in many patients with epilepsy and anxiety. ${ }^{27,28}$ In addition, focal epilepsies (in other structures) may include focal seizures without impaired awareness of which the conscious experience - and not understanding this experience - might be more frightening for someone with ID or lead to a fear of injury.

Lower levels of anxiety were associated with a high drug load of mood stabilizing AEDs.

Most probably this is due to the combination of the use of both multiple AEDs and the therapeutic properties of the described mood-stabilizing AEDs. Remarkably, anxiety appeared to be also related to a higher seizure frequency, even when the use of benzodiazepines as AED (daily or as rescue medication) was taken into account. This is in contrast with recent findings by Dehn et al., ${ }^{29}$ who demonstrated that a higher seizure frequency was significantly correlated with anxiety in patients with difficult-to-treat epilepsy (but without cognitive impairments) admitted to an epilepsy center. This difference might be explained by the fact that all our patients are living at a tertiary epilepsy center and that their seizure frequency is higher than in the study by Dehn et al. ${ }^{29}$ It could be hypothesized that the patients are therefore more habituated to having seizures and to its consequences in daily life. Also, professional caregivers who are specialized in epilepsy are always in close proximity, and provide care and attention to patients who have seizures. Such environmental factors were beyond the scope of this study, but should be included in future research.

Despite a relative high number of patients having elevated levels of depressive symptoms, none of the epilepsy characteristics were found to be significantly related. This seems to conflict with some studies on epilepsy and depression in patients without ID, suggesting that depression is associated with epilepsy-related characteristics. ${ }^{29,30}$ However, a review study by Hoppe and Elger concluded that the overall evidence is weak if stress-related epilepsy factors, such as social stigma, are taken into account. ${ }^{31}$ A social stigma might be less prominent in this sample, as the majority of patients live together with other patients with epilepsy and are therefore less "distinguished" from other members of the society. Furthermore, knowledge about the pathogenic mechanisms in the relationships between epilepsy, depression, and anxiety has been increasing. ${ }^{32}$ Future research is necessary to determine to what 
extent the underlying pathophysiology of epilepsy, depression, and anxiety applies to people with ID.

Irrespective of the epilepsy, this study demonstrated that ID characteristics were significantly associated with depressive and anxiety symptoms. With respect to the association between a more severe level of ID and depressive symptoms, it should be considered that the corresponding ADAMS subscale includes several somatic symptoms, such as fatigue and lack of energy. People with a more severe ID and epilepsy often are part of a frail population with multiple health problems, ${ }^{33}$ which might imply an increased vulnerability in this subgroup. Individuals who had an ID domain discrepancy, indicating that one domain of adaptive functioning is considerably more or less deficient than the other(s), had significantly higher depressive and anxiety levels than those without. Especially the practical and social abilities were more impaired in those with more depressive symptoms and anxiety, respectively. Although this concept of discrepancy requires further investigation, one could hypothesize that people with an ID domain discrepancy might be confronted with their (physical) limitations or are at risk of being overestimated by others, which may lead to feelings of frustration or stress.

Finally, despite the small subsample size, our findings confirm that a more severe epilepsy is associated with poorer quality of life regarding the domain of psychological functioning, which seems to emphasize the burden of epilepsy in their daily life. The quality of life was also significantly poorer in those with an ID domain discrepancy. There was, however, no direct association between the self-reported quality of life and the depressive and anxiety levels. This might be due to measurement aspects, such as the difference in informant (selfreport versus report by proxy) and the time span (current situation versus past six months).

\subsection{Limitations}

Of course this cross-sectional study is not without limitations. The study describes a specific (highly complex) population, with a highly frequent use of psychotropic medication (41.8\% of the 189 participants versus $14.0 \%$ of the 51 non-participants; $\mathrm{p}<0.001)$. Findings concerning QoL are based on a small sub-sample size of people with a mild ID, as the QoL self-report required a certain amount of verbal and cognitive abilities. Co-morbid psychiatric diagnosis such as autism or hyperkinetic disorders and etiological influences where beyond the scope of this present study. This will be part of future research in our centre, as we know that in general they often are accompanied by a higher level of anxiety and depressive symptoms. 


\subsection{Conclusions}

To conclude, this study emphasizes the relevance of depressive and anxiety symptoms in patients with epilepsy and ID, although the representability of our sample is limited to patients with primarily severe epilepsy who rely on tertiary epilepsy care facilities. Whereas anxiety and perceived quality of life are associated with specific epilepsy characteristics, depressive symptoms were only related to ID characteristics. These aspects should be taken into consideration by professionals working with this vulnerable population, so that they can anticipate on such comorbidities and contribute to good clinical care. 


\section{References}

1. Scott AJ, Sharpe L, Hunt C, et al. Anxiety and depressive disorders in people with epilepsy: A metaanalysis. Epilepsia 2017;58:973-982.

2. World Health Organization. Depression and Other Common Mental Disorders. Global Health Estimates. Geneva, Switzerland: WHO Document Production Services; 2017.

3. Robertson J, Hatton C, Emerson E, et al.. Prevalence of epilepsy among people with intellectual disabilities: A systematic review. Seizure 2015;29:46-62.

4. Kanner AM, Schachter SC, Barry JJ, et al. Depression and epilepsy: epidemiologic and neurobiologic perspectives that may explain their high comorbid occurrence. Epilepsy Behav 2012;24:156-168.

5. Peterson $\mathrm{CL}$, Walker $\mathrm{C}$, Shears $\mathrm{G}$. The social context of anxiety and depression: exploring the role of anxiety and depression in the lives of Australian adults with epilepsy. Epilepsy Behav 2014;34:29-33.

6. Reisinger EL, Dilorio C. Individual, seizure-related, and psychosocial predictors of depressive symptoms among people with epilepsy over six months. Epilepsy Behav 2009;15:196-201.

7. Van Ool JS, Snoeijen-Schouwenaars FM, Schelhaas HJ, et al. A systematic review of neuropsychiatric comorbidities in patients with both epilepsy and intellectual disability. Epilepsy Behav 2016;60: 130-137.

8. Espie CA, Watkins J, Curtice L, et al. Psychopathology in people with epilepsy and intellectual disability; an investigation of potential explanatory variables. J Neurol, Neurosurg Psychiatry 2003;74:1485-1492.

9. Kerr M, Linehan C, Thompson R, et al. A White Paper on the medical and social needs of people with epilepsy and intellectual disability: The Task Force on Intellectual Disabilities and Epilepsy of the International League Against Epilepsy. Epilepsia 2014;55:1902-1906.

10. Endermann M. Predictors of health-related and global quality of life among young adults with difficult-to-treat epilepsy and mild intellectual disability. Epilepsy Behav 2013;26:188-195.

11. Fisher RS, Acevedo C, Arzimanoglou A, et al. ILAE official report: a practical clinical definition of epilepsy. Epilepsia 2014;55:475-482.

12. American and P. Association, Diagnostic and Statistical Manual of Mental Disorders (Fifth ed.), ed. Arlington and VA. 2013: American Psychiatric Publishing.

13. Hermans $\mathrm{H}$, Jelluma $\mathrm{N}$, Van der Plas $\mathrm{FH}$, et al. Feasibility, reliability and validity of the Dutch translation of the Anxiety, Depression And Mood Scale in older adults with intellectual disabilities. Res Dev Disabil 2012;33:315-323.

14. Esbensen AJ, Rojahn J, Aman MG, et al. Reliability and validity of an assessment instrument for anxiety, depression, and mood among individuals with mental retardation. J Autism Dev Disord 2003;33:617-629.

15. Hamers PCM, Van Ool JS, Festen DAM, et al. Hermans H. Reliability and validity of the Dutch Anxiety, Depression And Mood Scale (ADAMS) in adults aged $<50$ years with intellectual disabilities. J Appl Res Intellect Disabil; 2018; 20

16. Hoekman J, Douma JCH, Kersten MCO, et al. IDQOL - Intellectual Disability Quality Of Life: De ontwikkeling van een instrument ter bepaling van de 'kwaliteit van bestaan' van mensen met een verstandelijke handicap. Nederlands Tijdschrift voor de Zorg aan Verstandelijk Gehandicapten 2001;27:207-225.

17. de Baaij EJJ, Hoekman J, Volman MJM, et al. Kwaliteit van bestaan bij mensen met een complex meervoudige beperking. Een bepaling met de IDQOL-16. Nederlands Tijdschrift voor de Zorg aan Mensen met Verstandelijke Beperkingen 2006;32:13-26.

18. Roeden MR, Maaskant MA, Bannink FP, et al. Solution-Focused Brief Therapy With People With Mild Intellectual Disabilities: A Case Series. J Policy Pract Intellec Disabil 2011;8:247-255.

19. Scheffer IE, Berkovic S, Capovilla G, et al. ILAE classification of the epilepsies: Position paper of the ILAE Commission for Classification and Terminology. Epilepsia 2017;58:512-521.

20. Leunissen CL, de la Parra NM, Tan IY, et al. Antiepileptic drugs with mood stabilizing properties and their relation with psychotropic drug use in institutionalized epilepsy patients with intellectual disability. Res Dev Disabil 2011;32:2660-2668. 
21. WHO Collaborating Centre for Drug Statistics Methodology. ATC/DDD index 2018. Available at: http://www.whocc.no/atcddd/. Accessed March 1, 2018.

22. American Psychiatric Association. Diagnostic and Statistical Manual of Mental Disorders (Fifth ed.). Arlington, VA: American Psychiatric Publishing; 2013.

23. Van Ool JS, Snoeijen-Schouwenaars FM, Tan IY, et al. Challenging behavior in adults with epilepsy and intellectual disability: an analysis of epilepsy characteristics. Epilepsy Behav 2018;86:72-78.

24. Hermans H, Evenhuis HM. Handleiding Angst, Depressie en Stemming Schaal voor mensen met een verstandelijke beperking. Rotterdam, The Netherlands: Geneeskunde voor Verstandelijk Gehandicapten, Erasmus MC; 2013.

25. Brandt C, Schoendienst M, Trentowska $M$, et al. Prevalence of anxiety disorders in patients with refractory focal epilepsy--a prospective clinic based survey. Epilepsy Behav 2010;17:259-63.

26. Kimiskidis VK, Triantafyllou NI, Kararizou E, et al. Depression and anxiety in epilepsy: the association with demographic and seizure-related variables. Ann Gen Psychiatry 2007;6:28.

27. de Oliveira GN, Kummer A, Salgado JV, et al. Psychiatric disorders in temporal lobe epilepsy: an overview from a tertiary service in Brazil. Seizure 2010;19:479-484.

28. Jones JE, Bell B, Fine J, et al. A controlled prospective investigation of psychiatric comorbidity in temporal lobe epilepsy. Epilepsia 2007;48:2357-2360.

29. Dehn LB, Pfäfflin M, Brückner S, et al. Relationships of depression and anxiety symptoms with seizure frequency: Results from a multicenter follow-up study. Seizure 2017;53:103-109.

30. Piazzini A, Canevini MP, Maggiori G, et al. Depression and Anxiety in Patients with Epilepsy. Epilepsy Behav 2001;2:481-489.

31. Hoppe C, Elger CE. Depression in epilepsy: a critical review from a clinical perspective. Nat Rev Neurol $2011 ; 7: 462-472$.

32. Kwon OY, Park SP. Depression and anxiety in people with epilepsy. J Clin Neurol 2014;10:175-188.

33. van Timmeren EA, van der Schans CP, van der Putten AA, et al. Physical health issues in adults with severe or profound intellectual and motor disabilities: a systematic review of cross-sectional studies. J Intellect Disabil Res 2017;61:30-49. 

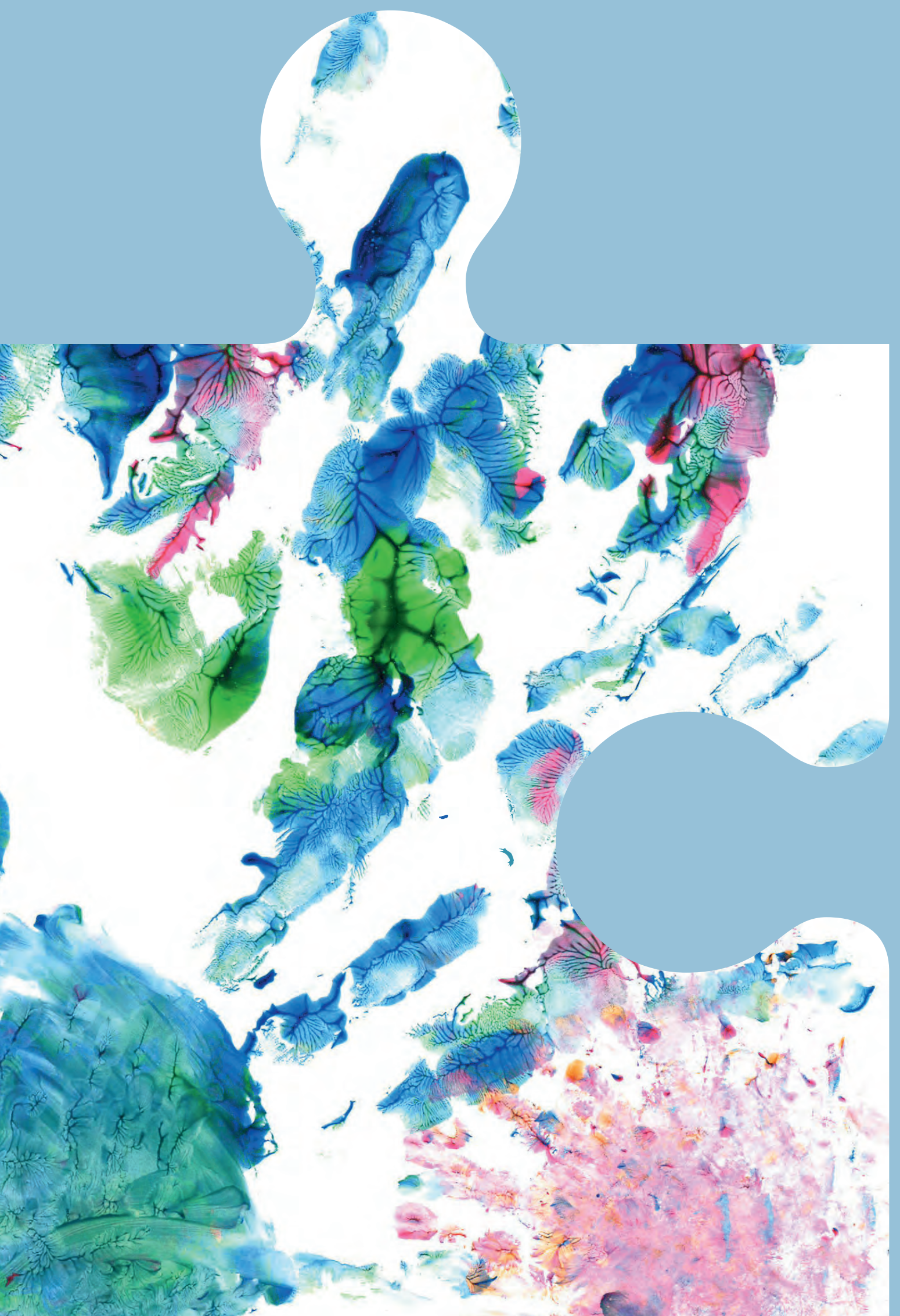


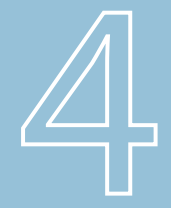

0

\section{Psychiatric evaluation of patients with epilepsy and ID: challenges and outcomes}

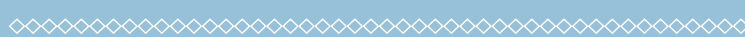

F.M. Snoeijen-Schouwenaars, J.S. van Ool, L. Evers, P.J.L. Collin, I.Y.Tan, A.P. Aldenkamp, J.G.M. Hendriksen, H.J. Schelhaas 


\section{Abstract}

\section{Purpose}

The primary objective of this study is to describe a standardized approach for comorbid behavioral and psychiatric screening of patients with both intellectual disability (ID) and epilepsy. In addition, we evaluated the relevance of specific ID and epilepsy characteristics in those patients in whom psychiatric co-morbidity was identified.

\section{Methods}

Adult patients with both ID and epilepsy, who rely on tertiary epilepsy care, were included ( $\mathrm{N}=189)$. Psychiatric status was assessed by psychiatric observations, semistructured interviews in line with DSM-5 and additional standardized questionnaires. Epilepsy characteristics were retrieved from patients' medical charts and ID was determined using subscales of the Vineland II for the social and practical domains, and psychological instruments, including intelligence tests, for the conceptual domain.

\section{Results}

Classification according to DSM-5 was possible in $51.8 \%$ of the patients. The presence of a psychiatric classification was significantly associated with ID domain discrepancy (substantial difference between two domains of adaptive behavior), a focal epilepsy type, but negatively related to seizure frequency.

\section{Conclusion}

A standardized approach for the evaluation of behavioral and psychiatric functioning in patients with both epilepsy and ID will help to identify patients with this type of comorbidity. The identification of comorbid clinical psychiatric manifestations is an essential element of the new ILAE criteria and may help to improve the quality of life by offering adequate, tailor-made treatment options and support. 


\section{Introduction}

The lifetime risk of psychiatric disorders in people with an intellectual disability (ID) appears to be considerably higher than in the non-ID population. ${ }^{1}$ Estimates of the prevalence vary greatly, depending on definitions, criteria applied and patient characteristics. ${ }^{1-3}$ Recognition, screening and assessment of these disorders is complicated by the ID itself, communication difficulties and additional disabilities.

The prevalence of epilepsy among patients with ID is estimated at around $22.2 \%$ (95\% Cl 19.6-25.0), the prevalence rate increasing with severity of ID. ${ }^{4}$ This is substantially higher than the reported prevalence of $1 \%$ in the general European population. $^{5}$

Although an association between epilepsy, ID and mental health problems is often encountered in clinical practice, the link between epilepsy and psychiatric and behavioral co-morbidity in this population remains unclear ${ }^{6}$; few studies have explored this topic.

Results from a systematic review indicate that a more severe form of epilepsy might be a risk factor for psychiatric disorders, ${ }^{7}$ and Espie et al. concluded that psychiatric symptoms were most strongly related to epilepsy characteristics, such as seizure frequency and severity. ${ }^{8}$

Despite the obvious interest in this topic from policy and care perspectives ${ }^{9}$, the applicability of existing literature is hampered by inconsistent definitions of ID or epilepsy and the use of small or highly selected samples. Methods for assessing psychiatric classifications in the ID population are limited and often not adjusted to DSM- $5^{10}$, or lack information on the extent of detail within assessments, instruments or diagnostic criteria used. The lack of information regarding psychiatric and behavioral co-morbidity in patients with ID is striking, as psychiatric symptoms influence the quality of life and daily functioning of a population already known for their complex needs. ${ }^{11}$ In addition, the presence of mental and physical disorders ${ }^{3,12}$ may affect anti-epileptic drug (AED) treatment choices.

The primary aim of this study is to describe and evaluate a more standardized approach to performing a psychiatric assessment in a 'high-risk' sample of patients with ID and epilepsy. In addition, we will investigate whether specific epilepsy and ID characteristics are associated with the presence of a psychiatric classification in this population. 


\section{Materials and methods}

\subsection{Study design and participants}

This study had a cross-sectional design and was part of the TRIANGLE study (The Relation between epilepsy, ID, And Neuropsychiatric comorbidities in a Group of patients in Long-term care for Epilepsy), which was conducted amongst in- and outpatients who rely on the tertiary epilepsy care facilities of Kempenhaeghe, The Netherlands.

In order to be eligible for this study, a patient had to meet all of the following criteria:

1) age $\geq 18$ years

2) diagnosis of epilepsy according to the clinical definition by the International League Against Epilepsy ${ }^{13}$

3) diagnosis of ID or current adaptive functioning at level of ID.

Clinical data on (demographic) characteristics were collected from the electronic patient files. This study is approved by the medical-ethical committee of Kempenhaeghe (No. 15.01) and the medical-ethical committee of Erasmus University Medical Center (MEC-2016-408).

\subsection{Instruments and procedure}

\subsubsection{Psychiatric evaluation}

The primary aim of this study was to describe the classification of possible psychiatric manifestations, not to be confused with a diagnosis according to strict diagnostic criteria. We designed structured case vignettes, which were evaluated by a physician for people with ID according to DSM-5 criteria, using a standardized stepwise approach for the classification process (see flowchart in Figure 4.1).

A psychiatric mental state examination was conducted using semi-structured interviews or patient observations (depending on the verbal communication skills of the patient) by the physician for people with ID (F.S). By means of questionnaires, additional information was acquired on psychiatric symptoms, including the Anxiety, Depression and Mood Scale (ADAMS) ${ }^{14}$ and the Behavioral Problem Inventory (BPI). ${ }^{15}$

Case vignettes, written by the first author, were created for each participant. Each vignette included information on demographics, level of ID, epilepsy and seizure type, chronic medical conditions, the mental state examination, life-long (psychiatric) history, results of the additional questionnaires, and a description of visual and auditory functioning. 


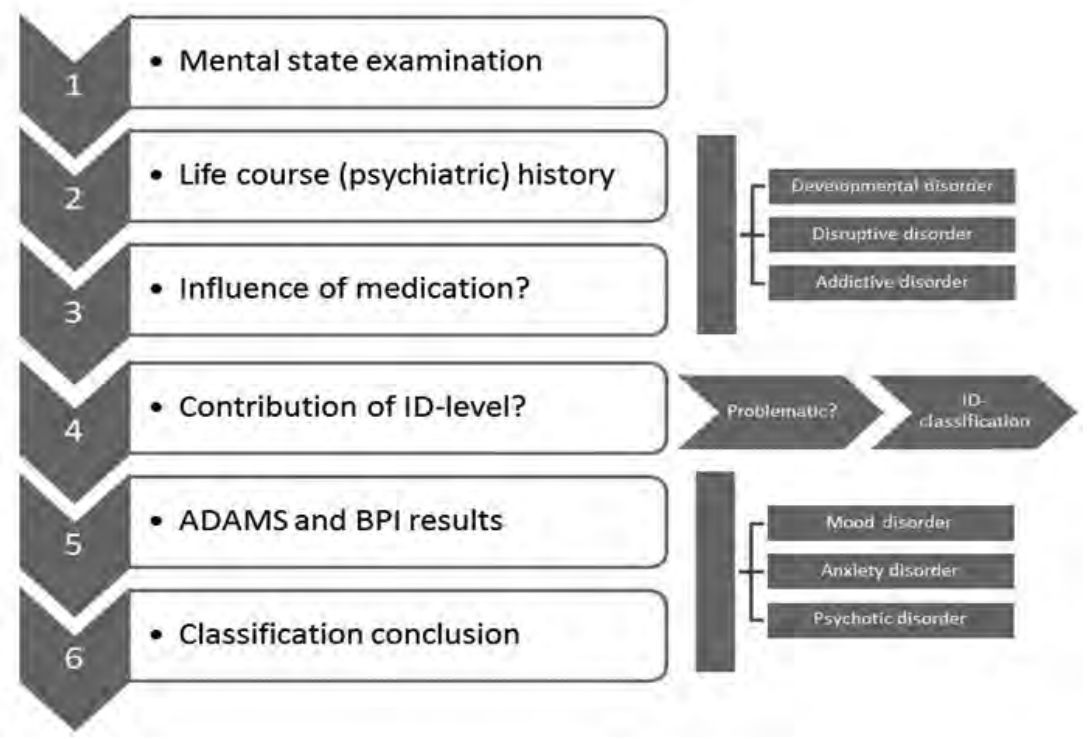

Figure 4.1: Flowchart: stepwise approach leading to a psychiatric classification of patients with ID. Note: in $1,2,3$ and/or 4, decide whether this diagnosis can explain other symptoms. If yes, make no other diagnosis. If it is clear that a disorder was present but is being effectively treated with drugs, note "under control".

The (diagnostic) classification categories (appendix I) are in accordance with the Psychiatric Assessment Schedule for Adults with Developmental Disabilities (PASADD) which has been used in previous studies. ${ }^{16}$ Patients were only eligible for the classification category "behavior only attributable to ID" when their behavior was of such intensity that it (a) required intervention and/or treatment, (b) hampered their daily routines, and when the intellectual disability was the only, and direct cause of these problems.

In order to assess the reliability of this method, the first 50 of the 189 included case vignettes were, in addition, independently rated by two senior consultant psychiatrists specialized in ID (R.E and P.C.). A number of cases $(n=19)$ in whom initial rating resulted in different classification categories, was further discussed during a consensus meeting between the two psychiatrists. Consensus was ultimately reached in all patients. The initial inter-rater agreement between the two psychiatrists, and between the psychiatrists' consensus and the classification by the ID-physician was measured using Cohen's kappa coefficient. ${ }^{17}$

In patients with a known etiology, we explored whether the determined individual psychiatric classification could be matched with the behavioral phenotype described 
in the literature, using specific etiology databases such as OMIM (Online Mendelian Inheritance in Man) and PubMed. We applied 3 categories of evidence:

1) "matching", when the same psychiatric comorbidity was described in the clinical synopsis of a specific etiology in OMIM.

2) "not matching", when no literature could be found

3) "possibly matching", when the psychiatric comorbidity was only mentioned in case reports or results were contradictory in the literature.

\subsubsection{Intellectual Disability}

We examined the overall level of ID and the presence of an ID domain discrepancy. The level of ID was based on the three domains of adaptive deficits as described in DSM-5: the conceptual, social and practical domains. ${ }^{10}$ Each domain was assessed separately using standardized instruments, such as subscales of the Vineland ${ }^{18}{ }^{18}$ for the social and practical domains, and psychological instruments, including intelligence tests, for the conceptual domain. Results were converted into a classification of mild, moderate, severe or profound deficits. An ID profile was considered discrepant when there was a substantial intra-individual difference between two DSM-5 domains, indicating that one domain was considerably more or less deficient than the other(s). For more information regarding the assessment of level of ID and domain discrepancy, see Van Ool et al. ${ }^{19}$

\subsubsection{Epilepsy characteristics}

Epilepsy characteristics were retrieved from the patient's medical records and included epilepsy type, age at onset, number of different seizure types, ${ }^{20}$ number of seizures in the past year, etiology and the prescribed AED. The classification of epilepsy was performed by a specialized neurologist (HJS). Seizures were recorded by the nursing staff. The epilepsy type and etiology were classified according to the most recent classification system by the ILAE. ${ }^{20}$ As a measure of drug load of AED with mood stabilizing properties, i.e. carbamazepine, valproic acid and lamotrigine, the prescribed daily dose versus defined daily dose (PDD/DDD) ratio was calculated. ${ }^{21}$ The DDDs were retrieved from the database of the WHO Collaborating Centre for Drug Statistics Methodology. ${ }^{22}$

The brain MRI findings were broadly categorized into 3 categories: normal, abnormal without epileptogenic focus, abnormal with epileptogenic focus. The allocation was based on the likelihood that the lesions were responsible for the seizures presented in the individual patient. If, based on the MRI location or characteristics, a lesion could theoretically be epileptogenic but could not be linked 
to the seizure type of this specific patient, it was rated as abnormal but not epileptogenic.

\subsection{Statistical analyses}

The level of agreement between the psychiatrists, and the agreement between the psychiatrist's consensus decision and the ID-physician for the psychiatric classifications (inter-rater reliability) was investigated Via Cohen's kappa. ${ }^{17}$ The kappa values were interpreted as follows: $k=0$ to 0.20 'none', 0.21 to 0.39 'minimal', 0.40 to 0.59 'weak', 0.60 to 0.79 'moderate', 0.80 - 0.90 'strong' and over 0.90 'almost perfect'. ${ }^{23}$

Multiple binary logistic regression analyses were performed to examine direct associations between predictors and the presence of a psychiatric classification, adjusting for the use of psychotropic medication, gender and age. In addition, multivariate logistic regression analyses were conducted to examine associations between the psychiatric classification category and all predictors, controlling for the use of psychotropic medication.

All analyses were conducted in IBM SPSS Statistics version 24.

\section{Results}

\subsection{Sample characteristics}

A total of 240 patients were invited to participate in the study, 189 of whom gave consent (inclusion rate: $78.8 \%$ ). This consent was provided by patients and/or their legal guardian when appropriate. The sample comprised $58.7 \%$ males with a mean age of 47.9 years ( $S D=15.6$; range 18.3-85.9 years). The majority of subjects lived in residential facilities (76.2\%); the others in community settings. The level of ID was mild in $20.1 \%$, moderate in $30.7 \%$, severe in $29.1 \%$, and profound in $20.1 \%$, and an ID domain discrepancy was present in $32.8 \%$. All other demographic and clinical characteristics are displayed in Table 4.1. 
Table 4.1: Demographic and clinicalcharacteristic

\begin{tabular}{|c|c|}
\hline Characteristics & Values \\
\hline Age at onset of epilepsy (years) & $M d n=2.0, I Q R=0-5.5$, range $0-53$ \\
\hline Infancy (<1 yr) & $32.8 \%$ \\
\hline Childhood (1-12 yr) & $54.0 \%$ \\
\hline Adolescence (12-18 yr) & $10.1 \%$. \\
\hline Adulthood $(18+y r)$ & $3.2 \%$ \\
\hline \multicolumn{2}{|l|}{ Epilepsy type $^{a}$} \\
\hline Generalized only & $10.6 \%$ \\
\hline Focal only & $41.3 \%$ \\
\hline Both generalized and focal & $44.4 \%$ \\
\hline Unknown & $3.7 \%$ \\
\hline Number of seizure types (semiology) ${ }^{a}$ & $M d n=3.0, I Q R=1-4$, range $0-8$ \\
\hline Seizure frequency (last year) & $M d n=70.0, I Q R=11.5-153.0$, range $0-1206$ \\
\hline Seizure-free & $12.7 \%$ \\
\hline Yearly & $12.2 \%$ \\
\hline Monthly & $19.6 \%$ \\
\hline Weekly & $43.9 \%$ \\
\hline Daily & $11.6 \%$ \\
\hline \multicolumn{2}{|l|}{ Etiology of epilepsy ${ }^{a}$} \\
\hline Structural & $28.6 \%$ \\
\hline Genetic & $20.1 \%$ \\
\hline Infectious & $6.3 \%$ \\
\hline Metabolic & $1.1 \%$ \\
\hline Unknown & $43.9 \%$ \\
\hline Daily use of anti-epileptic drugs & $M d n=3.0, I Q R=2.5-4.0$, range $0-6$ \\
\hline Daily use of psychotropic drugs & $41.8 \%$ \\
\hline Health problems & $79.9 \%$ \\
\hline Psychiatrist involved & $37.0 \%$ \\
\hline \multicolumn{2}{|l|}{ MRI } \\
\hline Not performed & $27.0 \%$ \\
\hline Normal & $22.8 \%$ \\
\hline Abnormal with possible epileptogenic focus & $43.4 \%$ \\
\hline Abnormal with no epileptogenic focus & $6.9 \%$ \\
\hline \multicolumn{2}{|l|}{ Sensory problems } \\
\hline None & $59.8 \%$ \\
\hline Visual problems & $28.0 \%$ \\
\hline Auditory problems & $4.2 \%$ \\
\hline Both auditory and visual problems & $7.9 \%$ \\
\hline
\end{tabular}

a Based on ILAE 2017 criteria. ${ }^{20}$ ID=intellectual disability; Mdn = median; IQR = interquartile range.

\subsection{Psychiatric evaluation}

The inter-rater reliability between the psychiatrist's consensus and the classification by the ID-physician was strong $(K=0.888)$. The initial inter-rater reliability between the psychiatrists regarding the presence of a psychiatric classification in general, was moderate $(K=0.774)$. A DSM- 5 based psychiatric classification was made in $51.9 \%$ of the patients. Details of the consensus classification are shown in Table 4.2. 
Of those with a psychiatric classification, a psychiatrist was involved in the patient care in $61.2 \%$ compared to $11.0 \%$ of those without a psychiatric classification $\left(x^{2}=51.06, p<0.001\right)$. We found that the patients with a psychiatric classification used psychotropic medication significantly more often than those without $(69.4 \%$ versus $\left.12.1 \%, X^{2}=63.68, p<0.001\right)$.

No association was found between having a psychiatric classification and sensory problems $(p=0.108)$ or general health problems $(p=0.258)$.

In $11.6 \%$ the etiology of the patient also included the described psychiatric classification.

Table 4.2: Psychiatric classifications.

\begin{tabular}{lc}
\hline Psychiatric classification & $48.1 \%$ \\
None & $12.7 \%$ \\
Mood disorders & $4.2 \%$ \\
Anxiety disorders & $1.6 \%$ \\
Psychotic disorders & $0.5 \%$ \\
ADHD & $25.4 \%$ \\
ASD & $4.8 \%$ \\
Behavior only attributable to ID & $1.6 \%$ \\
Behavioral disorders & $1.1 \%$ \\
Other & \\
Psychiatric classification category & $48.1 \%$ \\
None & $30.7 \%$ \\
Neurodevelopmental (ASD+ADHD+ID) & $16.9 \%$ \\
Affective (mood + anxiety disorder) & $4.2 \%$ \\
Other & \\
Behavioral phenotype matching etiology & $76.2 \%$ \\
No classification or unknown etiology & $4.8 \%$ \\
Not matching etiology & $11.6 \%$ \\
Matching etiology & $7.4 \%$ \\
Possibly matching etiology & \\
\hline
\end{tabular}

$I D=$ intellectual disability; $A D H D=$ attention deficit hyperactivity disorder ${ }_{\text {, }}$ ASD = autism spectrum disorder.

\subsection{Potential risk- and associated factors}

Unadjusted logistic regression analyses, examining the direct associations between a psychiatric classification and each predictor, revealed the likelihood of having a psychiatric classification was significantly higher if the subject had focal epilepsy $(\mathrm{OR}=2.78, \mathrm{p}=0.047)$, an ID domain discrepancy $(\mathrm{OR}=2.96, \mathrm{p}=0.001)$ or a mild or moderate level of ID $(O R=1.85, p=0.036)$. Details of these analyses are presented in Table 4.3.

While adjusting for age, gender and the use of psychotropic medication, the likelihood of having a psychiatric classification was significantly higher for those with focal epilepsy $(O R=6.45, p=0.011)$ and for those with an ID domain discrepancy 
$(\mathrm{OR}=3.10, \mathrm{p}=0.018)$. It was significantly lower for those with a higher seizure frequency $(\mathrm{OR}=0.97, \mathrm{p}=0.045)$.

Post hoc chi-square analyses were conducted in order to investigate which ID domain was most often impaired in subjects with a psychiatric classification. The results show that an ID domain discrepancy, with the social domain being particularly impaired, was most often present in those with a psychiatric classification (in $26.5 \%$ of cases; $\left.X^{2}=7.39, p=0.007\right)$.

Table 4.3: Logistic regression analyses predicting presence of a psychiatric classification.

\begin{tabular}{lcccccc}
\hline & \multicolumn{3}{c}{ Unadjusted analyses } & \multicolumn{2}{c}{ Adjusted analyses $^{\mathbf{a}}$} \\
\cline { 2 - 7 } & B (SE) & OR & $\mathbf{9 5 \% - C I}$ & B (SE) & OR & $\mathbf{9 5 \% - C I}$ \\
\hline Psychiatric classification & & & & & & \\
Number of seizure types & $-0.03(0.08)$ & 0.97 & $0.84-1.13$ & $0.39(0.17)$ & 1.04 & $0.74-1.45$ \\
Seizure frequency & $-0.01(0.01)$ & 0.99 & $0.97-1.01$ & $-0.03(0.02)$ & $0.97^{*}$ & $0.94-0.9$ \\
Focal epilepsy & $1.02(0.51)$ & $2.78^{*}$ & $1.02-7.58$ & $1.86(0.74)$ & $6.45^{*}$ & $1.52-27.35$ \\
Drug load mood-stabilizing AED & $-0.12(0.20)$ & 0.89 & $0.60-1.32$ & $-0.46(0.28)$ & 0.63 & $0.36-1.10$ \\
ID discrepancy & $1.09(0.33)$ & $2.96^{* *}$ & $1.56-5.64$ & $1.13(0.48)$ & $3.10^{*}$ & $1.22-7.87$ \\
Level ID (mild/moderate) & $0.62(0.30)$ & $1.85^{*}$ & $1.04-3.30$ & $-0.11(0.52)$ & 0.89 & $0.32-2.49$ \\
\hline
\end{tabular}

${ }^{*} \mathrm{p}<0.05$, ${ }^{* *} \mathrm{p}<0.01$. The adjusted multivariate models also included age, gender, and use of psychotropic drugs as predictors. $\mathrm{B}=$ beta; $\mathrm{SE}=$ standard error; $\mathrm{OR}=$ odds ratio; $\mathrm{Cl}=$ confidence interval; $\mathrm{AED}=$ anti-epileptic drugs; ID = intellectual disability.

Table 4.4: Multiple logistic regression analyses predicting categories of a psychiatric classification.

\begin{tabular}{lcccc}
\hline & B (SE) & OR & $\mathbf{9 5 \% - C I}$ & p \\
\hline Neurodevelopmental & & & & .276 \\
Number of seizure types & $-0.18(0.17)$ & 1.20 & $0.87-1.66$ & .261 \\
Seizure frequency & $-0.02(0.02)$ & 0.98 & $0.95-1.01$ & $.013^{*}$ \\
Focal epilepsy & $2.25(0.91)$ & 9.51 & $1.60-56.67$ & .433 \\
Drug load mood-stabilizing AED & $-0.23(0.30)$ & 0.79 & $0.44-1.42$ & $.013^{*}$ \\
ID discrepancy & $1.15(0.49)$ & 3.16 & $1.21-8.25$ & .599 \\
Level ID (mild/moderate) & $-0.28(0.53)$ & 0.76 & $0.27-2.15$ & .392 \\
Affective & & & & .176 \\
Number of seizure types & $-0.42(1.09)$ & 1.18 & $0.81-1.72$ & .069 \\
Seizure frequency & $-0.03(0.02)$ & 0.97 & $0.92-1.01$ & $.039^{*}$ \\
Focal epilepsy & $1.60(0.88)$ & 4.93 & $0.88-27.50$ & $.046^{*}$ \\
Drug load mood-stabilizing AED & $-0.75(0.36)$ & 0.47 & $0.23-0.96$ & .353 \\
ID discrepancy & $1.12(0.56)$ & 3.06 & $1.02-9.15$ & $0.52-6.09$ \\
Level ID (mild/moderate) & $0.58(0.63)$ & 1.79 & & \\
\hline
\end{tabular}

The psychiatric classification category is compared to having no psychiatric classification. The analyses are adjusted for the use of psychotropic medication. ${ }^{*} \mathrm{p}<0.05 ; \mathrm{B}=$ beta; $\mathrm{SE}=$ standard error; $\mathrm{OR}=$ odds ratio; $\mathrm{Cl}=$ confidence interval; $\mathrm{AED}=$ anti-epileptic drugs; ID = intellectual disability. 


\section{Discussion and conclusions}

In this current study on 189 patients with both epilepsy and ID, we identified a psychiatric classification in $51.8 \%$ of the patients according to DSM-5. Although prevalence rates of psychiatric classifications in this population vary between different studies, this number is at the 'high-end' of the prevalence-spectrum. 1,2,24

As the patients were recruited from a tertiary referral center for epilepsy, the results cannot be completely generalized to the entire population of adults with ID and epilepsy. However, given the fact that, within this population, nearly eighty percent participated in the study, and considering the limited number of studies that focus on this specific population and address the complexity of the interaction between ID, epilepsy and psychiatry ${ }^{25}$, the study provides essential new information. The study is also in line with the new ILAE criteria ${ }^{20}$ stating that: "Like etiology, it is important that the presence of comorbidities are considered for every patient with epilepsy at each stage of classification, enabling early identification, diagnosis, and appropriate management."

When considering the challenges of describing psychiatric manifestations, there are a few aspects we wish to highlight: The mental state examination was a challenge, especially in the patients with a more severe ID, because it sometimes required describing a quite complicated internal, subjective feeling or cognition. In those with a more severe level of ID, we, therefore, assessed the communicating abilities, sensory impairments, memory and ability to concentrate as being of 'greater relevance'.

Life course history-taking in an adult population was difficult in some cases, as a substantial part of a person's early life might not be known, even though they have been receiving care for decades. This information is particularly important when trying to distinguish between behavioral disorders and, for instance, the presence of autistic spectrum characteristics. The evaluation of psychiatric disorders in people with a severe/profound ID is complicated, as the ID might "overshadow" psychiatric disorders and prevent the search for probable psychopathological symptoms. ${ }^{26}$ Although we tried to prevent this phenomenon, called 'diagnostic overshadowing', and increased the potential for identification of psychiatric symptoms by using both quantitative measures and direct observation, we cannot exclude the possibility that it might have influenced our results.

The fact that a psychiatric classification could be identified more frequently in those with mild/moderate ID compared to those with severe/profound ID, is in line with previous literature finding $\mathrm{s}^{27}$, but was only found in the unadjusted analyses in 
our cohort. When adjusting for age, gender and the use of psychotropic medication, the level of ID was not a potential risk-factor. That a psychiatric classification was more often present in those with an ID discrepancy, compared to those without, stresses the relevance of this relatively new diagnostic DSM-5 aspect and underlines that in this population, all domains of ID should be taken into account, not only the 'summarizing' level of ID-classification.

Current evidence suggests that most mental health problems decline with age (except for dementia). ${ }^{28}$ We have not specifically taken this decline or dementia into account in our study. This could be a direction for future research; for instance, sideeffects of medications may present differently due to pharmacodynamic and kinetic changes in older age; there may be higher rates of sensory impairments that increase communication difficulties and aging-specific disorders (such as Alzheimer's disease) may predispose persons with intellectual disability to psychiatric comorbidity.

A higher frequency of psychiatric classification was found among patients with a focal epilepsy. Although we did not include the supposed focus (temporal/extra temporal) of epilepsy in our analysis, the link between focal epilepsy and, for example, anxiety has also been described in previous studies ${ }^{29,30}$ and might be explained by the involvement of temporal brain structures..$^{31}$ In addition, one might speculate that in those patients with a focal but extratemporal form of epilepsy, a seizure type with retained awareness might be even more frightening for patients with an ID.

Intriguingly, a psychiatric classification appeared to be related to a lower seizure frequency, even when the use of mood-stabilizing AED was taken into account. This is counter-intuitive and contrasts with a publication by Dehn et al..$^{32}$ suggesting that in a group of patients without ID, a higher seizure frequency was significantly correlated with anxiety. Although we can only speculate, a possible explanation might be that most of the included patients relied on tertiary care facilities, leading to the close proximity of professional caregivers, who can provide direct care and attention. These environmental factors were beyond the scope of this study, but would be interesting to incorporate in future research.

We found that the patients with a psychiatric classification used psychotropic medication (69.4\%) significantly more often than those without. Despite these drugs, they still suffered from psychiatric symptoms. Although this may reflect the refractory nature of certain psychiatric symptoms, it also leads to the question whether they received the correct treatment for their symptoms. As we know from literature and previous studies, psychotropic drugs in general, and antipsychotics in particular, are 
overused in people with ID and are often prescribed for challenging behavior rather than for diagnosed mental illness, despite the lack of evidence of efficacy. ${ }^{9}$

In this study, we showed that a standardized approach for evaluating behavioral and psychiatric functioning among patients with both epilepsy and ID has its challenges, but can be useful in identifying patients and specific factors contributing to this co-morbidity. The identification of comorbid clinical psychiatric manifestations is an essential element of the new ILAE criteria and may help to improve the quality of life by offering adequate, tailor-made treatment options and support. 


\section{References}

1. Einfeld SL, Piccinin AM, Mackinnon A, Hofer SM, Taffe J, Gray KM, et al. Psychopathology in young people with intellectual disability. JAMA 2006;296:1981-9.

2. Cooper SA. Epidemiology of psychiatric disorders in elderly compared with younger adults with learning disabilities. Br J Psychiatry 1997;170:375-80.

3. Cooper SA, Smiley E, Morrison J, Williamson A, Allan L. Mental ill-health in adults with intellectual disabilities: prevalence and associated factors. Br J Psychiatry 2007;190:27-35.

4. Robertson J, Hatton C, Emerson E, Baines S. Prevalence of epilepsy among people with intellectual disabilities: A systematic review. Seizure 2015;29:46-62.

5. Forsgren L, Beghi E, Oun A, Sillanpää M. The epidemiology of epilepsy in Europe - a systematic review. Eur J Neurol 2005;12:245-53.

6. Matthews T, Weston N, Baxter H, Felce D, Kerr M. A general practice-based prevalence study of epilepsy among adults with intellectual disabilities and of its association with psychiatric disorder, behaviour disturbance and carer stress. J Intellect Disabil Res 2008;52:163-73.

7. van Ool JS, Snoeijen-Schouwenaars FM, Schelhaas HJ, Tan IY, Aldenkamp AP, Hendriksen JGM. A systematic review of neuropsychiatric comorbidities in patients with both epilepsy and intellectual disability. Epilepsy Behav 2016;60:130-7.

8. Espie CA, Watkins J, Curtice L, Espie A, Duncan R, Ryan JA, et al. Psychopathology in people with epilepsy and intellectual disability; an investigation of potential explanatory variables. J Neurol Neurosurg Psychiatry 2003;74:1485-92.

9. Sheehan R, Hassiotis A, Walters K, Osborn D, Strydom A, Horsfall L. Mental illness, challenging behaviour, and psychotropic drug prescribing in people with intellectual disability: UK population based cohort study. BMJ 2015;351:h4326.

10. American Psychiatric Association. Diagnostic and Statistical Manual of Mental Disorders (Fifth ed.). Arlington, VA: American Psychiatric Publishing 2013.

11. Kerr M, Linehan C, Thompson R, Mula M, Gil-Nagal A, Zuberi SM, et al. A White Paper on the medical and social needs of people with epilepsy and intellectual disability: the Task Force on Intellectual Disabilities and Epilepsy of the International League Against Epilepsy. Epilepsia 2014;55:1902-6.

12. Kwok H, Cheung PW. Co-morbidity of psychiatric disorder and medical illness in people with intellectual disabilities. Curr Opin Psychiatry 2007;20:443-9.

13. Fisher RS, Acevedo C, Arzimanoglou A, Bogacz A, Cross JH, Elger CE, et al. ILAE official report: a practical clinical definition of epilepsy. Epilepsia 2014;55:475-82.

14. Hermans $\mathrm{H}$, Jelluma $\mathrm{N}$, van der Pas $\mathrm{FH}$, Evenhuis HM. Feasibility, reliability and validity of the Dutch translation of the Anxiety, Depression And Mood Scale in older adults with intellectual disabilities. Res Dev Disabil 2012;33:315-23.

15. Rojahn J, Matson JL, Lott D, Esbensen AJ, Smalls Y. The Behavior Problems Inventory: an instrument for the assessment of self-injury, stereotyped behavior, and aggression/destruction in individuals with developmental disabilities. J Autism Dev Disord 2001;31:577-88.

16. Moss S, Prosser H, Costello H, Simpson N, Patel P, Rowe S, et al. Reliability and validity of the PAS-ADD Checklist for detecting psychiatric disorders in adults with intellectual disability. J Intellect Disabil Res 1998;42:173-83.

17. Cohen J, A coefficient of agreement for nominal scales. In: Educ Psychol Meas 1960: 37-46.

18. Sparrow SS, Cicchetti DV, Balla, AD. Vineland II: Vineland Adaptive Behavior Scales (2nd ed.). Minneapolis, MN: Pearson Assessments 2005.

19. van Ool JS, Snoeijen-Schouwenaars FM, Tan IY, Schelhaas HJ, Aldenkamp AP, Hendriksen JGM.Challenging behavior in adults with epilepsy and intellectual disability: An analysis of epilepsy characteristics. Epilepsy Behav 2018;86:72-8.

20. Scheffer IE, Berkovic S, Capovilla G, Connolly MB, French J, Guilhoto L, et al. ILAE classification of the epilepsies: Position paper of the ILAE Commission for Classification and Terminology. Epilepsia 2017;58:512-21. 
21. Leunissen CL, de la Parra NM, Tan IY, Rentmeester TW, Vader Cl, Veendrick-Meekes MJ, et al. Antiepileptic drugs with mood stabilizing properties and their relation with psychotropic drug use in institutionalized epilepsy patients with intellectual disability. Res Dev Disabil 2011;32:2660-8.

22. WHO, Methodology CCfDS. ATC/DDD index 2018 http://www.whocc.no/atcddd/ [accessed december 2018]

23. McHugh ML. Interrater reliability: the kappa statistic. Biochem Med (Zagreb) 2012;22:276-82.

24. Baumeister AA, Todd ME, Sevin JA. Efficacy and specificity of pharmacological therapies for behavioral disorders in persons with mental retardation. Clin Neuropharmacol 1993;16:271-94.

25. Smith KR, Matson JL. Psychopathology: differences among adults with intellectually disabled, comorbid autism spectrum disorders and epilepsy. Res Dev Disabil 2010;31:743-9.

26. Reiss S, Szyszko J. Diagnostic overshadowing and professional experience with mentally retarded persons. Am J Ment Defic 1983;87:396-402.

27. Hove O, Havik OE. Developmental level and other factors associated with symptoms of mental disorders and problem behaviour in adults with intellectual disabilities living in the community. Soc Psychiatry Psychiatr Epidemiol 2010;45:105-13.

28. Evenhuis HM, Hermans H, Hilgenkamp TI, Bastiaanse LP, Echteld MA. Frailty and disability in older adults with intellectual disabilities: results from the healthy ageing and intellectual disability study. J Am Geriatr Soc 2012;60:934-8.

29. Brandt C, Schoendienst M, Trentowska M, May TW, Pohlmann-Eden B, Tuschen-Caffier B, et al. Prevalence of anxiety disorders in patients with refractory focal epilepsy--a prospective clinic based survey. Epilepsy Behav 2010;17:259-63.

30. Kimiskidis VK, Triantafyllou NI, Kararizou E, Gatzonis SS, Fountoulakis KN, Siatouni A, et al. Depression and anxiety in epilepsy: the association with demographic and seizure-related variables. Ann Gen Psychiatry 2007;6:28.

31. Jones JE, Bell B, Fine J, Rutecki P, Seidenberg M, Hermann B. A controlled prospective investigation of psychiatric comorbidity in temporal lobe epilepsy. Epilepsia 2007;48:2357-60.

32. Dehn LB, Pfäfflin M, Brückner S, Lutz MT, Steinhoff BJ, Mayer T, et al. Relationships of depression and anxiety symptoms with seizure frequency: Results from a multicenter follow-up study. Seizure 2017;53:103-9. 


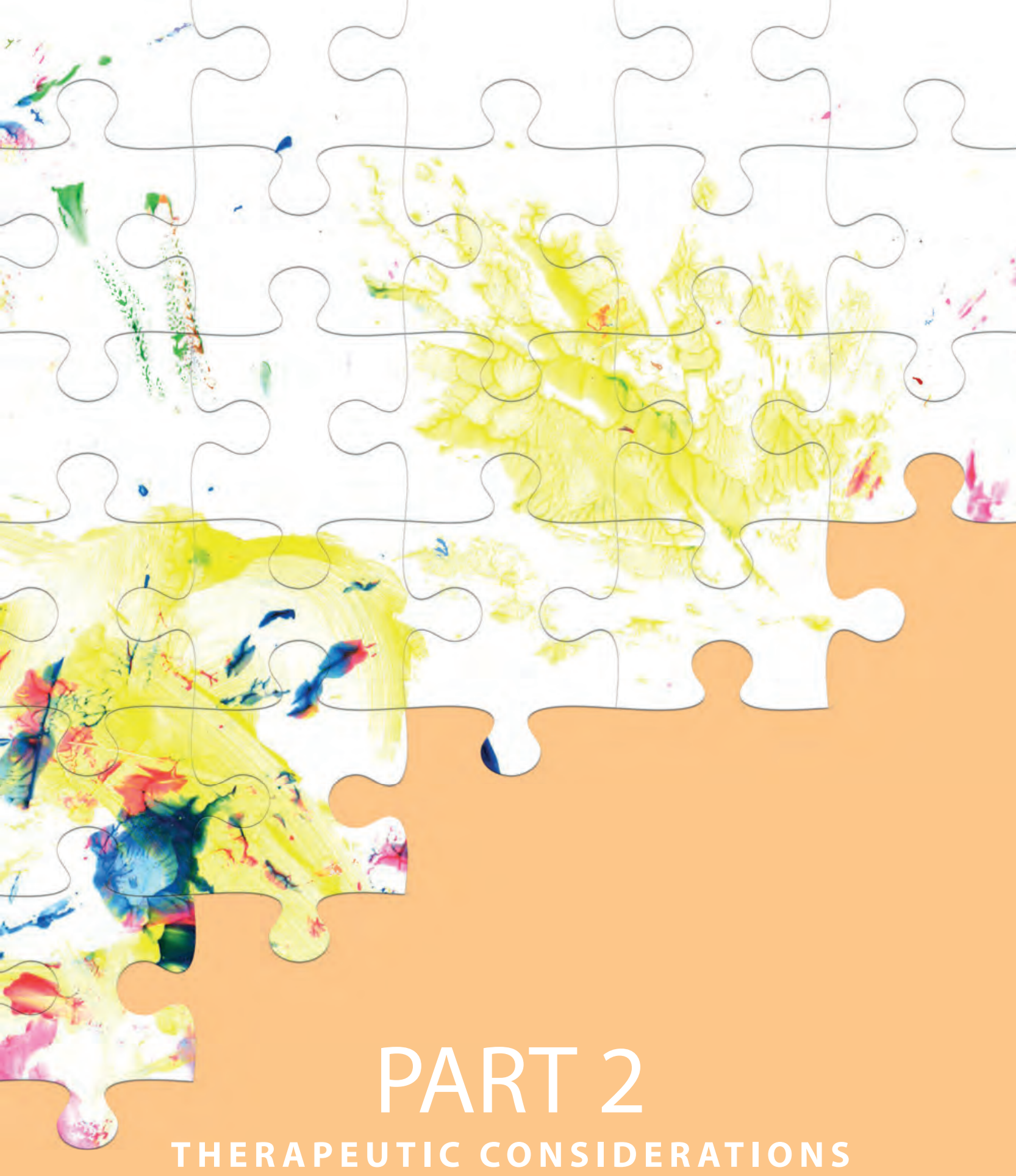




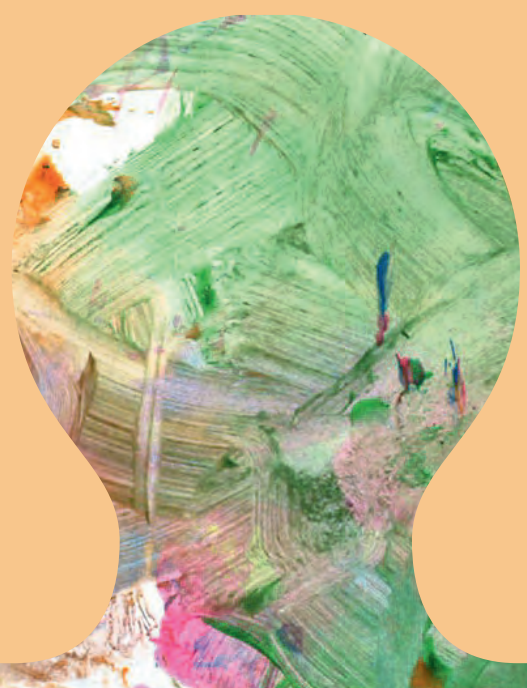

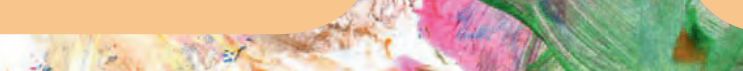

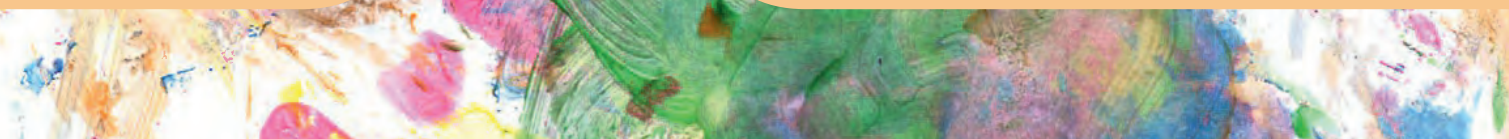

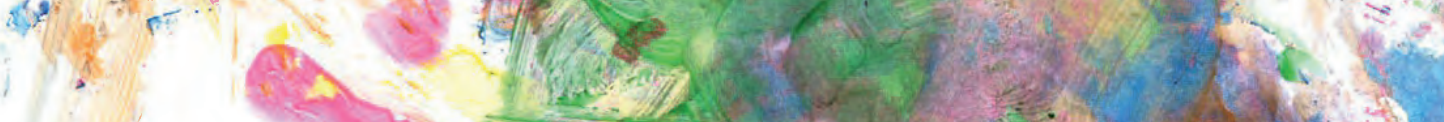




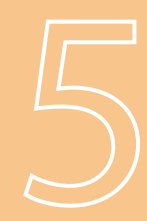

\section{Anti-epileptic drug treatment in patients with both epilepsy and ID: a tale of two services}

F.M. Snoeijen-Schouwenaars, C. Young, J. Palmer, C. Rowe, J.S.van Ool, M.H. Majoie, H.J. Schelhaas, R. Shankar. 


\section{Abstract}

\section{Objective}

Around a quarter of people with Intellectual Disability (ID) have epilepsy which is drug resistant in up to two-thirds. The prevalence of epilepsy, physical and behavioral problems increases with severity of ID. Little is known on prescribing practices to this population. We assessed similar cohorts of people with ID and epilepsy in two different countries to compare prescribing practices.

\section{Materials and methods}

We conducted a retrospective case-note review in a specialist ID community service in England and in a tertiary ID care facility in the Netherlands. We collected information on demographics, ID severity, genetic conditions, medical/behavioural/mental health co-morbidities, psychotropic and antiepileptic drugs (AEDs) for each cohort. Descriptive analysis, Pearson's Chi Squared, Fisher's Exact and Mann-Whitney U tests were used to explore possible associations. Statistical significance was accepted if $p<0.05$.

\section{Results}

The two cohorts were comparable in their baseline characteristics. Three-quarter of the Dutch was on three or more AEDs compared to a third in the English. The mean dosages between three most common AEDs prescribed were comparable. The Dutch had higher rates of physical comorbidity, less active mental or behavioral disorders but were more likely to be on anti-psychotic medication.

\section{Conclusion}

Despite the limitations of being an observational retrospective study involving two differing cohorts, our data indicates that individuals with ID and epilepsy have higher levels of physical and mental health co-morbidity and polypharmacy associated with increased severity of ID. 


\section{Introduction}

Intellectual disability (ID) is characterized by significant impairments of intellectual and adaptive functioning with onset before adulthood. ${ }^{1}$ These impairments are complicated by a significant presence of epilepsy, physical and mental comorbidities. $^{2,3}$ Epilepsy and ID may be caused by a range of pathological processes. ${ }^{4,-6}$ A quarter of people with ID have epilepsy and the prevalence rises with increasing levels of ID..$^{3,7}$ In those with a mild to moderate ID, epilepsy is present in up to $15 \%$ increasing up to half in those with profound ID. ${ }^{8}$

Two-thirds of people with ID and epilepsy do not respond to current anti-epileptic medication and is associated with high healthcare costs and premature mortality. ${ }^{7}$ Seizures are the most frequent potentially avoidable reason for hospital admissions in people with ID. ${ }^{9,10}$

Autism spectrum disorder is often seen in people with ID and epilepsy and is more common with severe ID. ${ }^{11}$ It is, however, challenging to outline relationships between autism, ID and epilepsy. ${ }^{12}$ Evidence suggests that the severity of ID is not only associated with an increased risk of epilepsy and autism but also increased polypharmacy. ${ }^{2}$ It has been reported that the more severe the ID, the more likely psychotropic drugs are prescribed. ${ }^{13}$ The ID Supplement of the Irish Longitudinal Study on Ageing (IDS-TILDA) reports that the presence of chronic conditions is likely to lead to the use of multiple medications. ${ }^{14}$ In their sample half of those with ID were either on antipsychotics or Antiepileptic Drugs (AEDs) and about a third were using them concomitantly. ${ }^{14}$

It is customary for AED prescribing guidance to emerge from prospective randomized control trials which often exclude people with ID and there is little data or none on how prescribing occurs in this population. ${ }^{15}$ Therefore the aim of this study was to evaluate the clinical management in two specialized services for epilepsy in people with complex forms of ID, in two countries of similar socioeconomic status.

\section{Materials and methods}

We used a retrospective case-note review design to gather information on people who satisfied the ILAE criteria of drug resistant epilepsy ${ }^{16}$ attending one of the two centres: Cornwall (the English cohort) and Kempenhaeghe (the Dutch cohort).

Cornwall is a county in the south west of England with a population of 600,000 people. It has a higher than average number of residential care facilities and 
thus have a larger number of people with epilepsy and $I^{17}$. This had led to the commissioning of a bespoke multi-disciplinary epilepsy service for adults with ID. The service sits within the County's mental and behavior health provider and is led by psychiatrists with specialized training in ID and epilepsy. ${ }^{18}$ Kempenhaeghe is one of the 2 specialized epilepsy centers in the Netherlands, offering multidisciplinary care management to people with epilepsy and ID. ${ }^{19}$ The service is led by neurologists and physicians who specialized in ID. Work in Cornwall was part of an audit looking at prescribing standards and registered with the local NHS Trust. In the Dutch sample residents had provided consent for the anonymous use of retrospective clinical data from their electronic health records for research purposes.

All people eligible and under follow up between 1st April and 30th June 2016 were enrolled. Clinical letters and electronic health records were reviewed. Information was gathered using a pre-designed data spread sheet. The following Information was gathered:

1. Demographics

2. Level of ID - this was divided as per the ICD or DSM criteria into 2 groups i.e. mild/moderate ID and 'severe/profound ID based on the rationale in Appendix 5.1.

3. Number of current AEDs and dosages of most commonly used AEDs.

4. Number of individuals with any chronic medical condition as evidenced by a recorded diagnosed condition in the individual's medical records.

5. Number of those experiencing mental or behavioral problems according to ICD 10 or DSM-IV diagnosis.

6. Number of those with Autism Spectrum Disorder (ASD) or other psychological disorders according to ICD 10 or DSM-IV criteria.

7. Number prescribed psychotropic medication (anti-psychotic and non-antipsychotic).

8. Number of those with a recorded genetic disorder

\subsection{Statistical analysis}

Data is presented descriptively in the form of summary measures by cohort (and total sample population) with suitable measures of variation as suitable. Pearson's Chi Squared or Fisher's Exact Test (as appropriate) was used to explore association between the ID and clinical factors. The Mann-Whitney U Test was used to explore the association between total number of AEDs and the two groups of mild-moderate ID and severe to profound ID. The tests to compare the two services treatment approaches were two-tailed and statistical significance was accepted if $p<0.05$. 


\subsection{Ethics}

The project was done using anonymised pooled data from the two centres. No patient data of each centre was shared with the other. Each centre had its own team statistician who did the analysis. This individual centre analysis was compared for the two centres and where appropriate further observations drawn from the anonymous combined data. Data had been collected as part of ongoing service evaluation and registered as such respectively with both organizations. Work in Cornwall UK was part of a registered NHS audit looking at prescribing standards and registered with the local NHS Trust. We also used the NHS Health research authority tool (http://www.hradecisiontools. org.uk/research/index.html) which helped confirm that no ethics is needed for this project.

In the Dutch sample patients had provided consent for the anonymous use of retrospective clinical data from their electronic health records for research purposes. In both centres data was collected separately by the respective clinicians of the services with whom the patient responsibility belonged. No author had access to any patient identifiable information other than to their own clients within their service. The two centres collected data separately and then compared their respective results. As there were acknowledged differences in the population, clinician practices and guidance (all discussed in the limitations section) the individual centre results were not considered generalizable.

\section{Results}

\subsection{Sample characteristics}

The English cohort consisted of 167 people (98 males; age range 18-73 years) and the Dutch cohort of 189 people (111 males; age range 18-85 years. In the English cohort 59 had a mild/moderate ID (35\%) and 108 had severe/profound ID (65\%); in the Dutch cohort 96 (51\%) had a mild/moderate level of ID and 93 a severe/profound level of ID (49\%). The English site had 33 people (19.5\%) on a Vagal Nerve Stimulator (VNS) in situ compared to the Dutch sample of $24(12.7 \%)$. None was on a dietary treatment such as the ketogenic diet. Almost all in both cohorts were Caucasian. All satisfied the criteria for drug resistant epilepsy. 


\subsection{Comparison of 2 services}

The summary of findings of the comparisons on comorbid physical, mental and/or behavioral conditions, ASD, genetic conditions, psychotropic and antipsychotic coprescribing between the two groups of ID in the cohorts are provided in Table 5.1. The comparison of the prescribed dosages of commonly used AEDs in the two cohorts is provided in Table 5.2. The mean dosages between samples were similar. Clobazam was prescribed in nearly half of people in Dutch (48\%) but only $13 \%$ of the English sample where it is mainly used as a rescue medication.

Table 5.1: Summary of findings of the comparisons between the two groups of ID in the UK and the Netherlands cohorts.

\begin{tabular}{|c|c|c|c|c|c|c|}
\hline & \multicolumn{3}{|c|}{ UK ( $N=167)$} & \multicolumn{3}{|c|}{ The Netherlands $(\mathrm{N}=189)$} \\
\hline & $\begin{array}{c}\text { Mild } / \\
\text { moderate ID } \\
\mathrm{n}=\mathbf{5 9}(35.3 \%)\end{array}$ & $\begin{array}{c}\text { Severe/ } \\
\text { profound ID } \\
n=108(64.7 \%)\end{array}$ & $\begin{array}{c}\text { Significance } \\
\text { (p-value) }\end{array}$ & $\begin{array}{c}\text { Mild } / \\
\text { moderate ID } \\
n=96(50.8 \%)\end{array}$ & $\begin{array}{c}\text { Severe/ } \\
\text { profound ID } \\
\mathrm{n}=93(49.2 \%)\end{array}$ & $\begin{array}{c}\text { Significance } \\
\text { (p-value) }\end{array}$ \\
\hline \multicolumn{7}{|l|}{ Gender } \\
\hline Male & $37(62.7 \%)$ & $61(56.4 \%)$ & & $54(56.3 \%)$ & $57(61.3 \%)$ & \\
\hline Female & $22(37.3 \%)$ & $47(43.5 \%)$ & & $42(43.8 \%)$ & $36(38.7 \%)$ & \\
\hline \multicolumn{7}{|l|}{ Age in years } \\
\hline Range & $18-73$ & $18-73$ & & $18-84$ & $19-85$ & \\
\hline Mean, median & $40.7,42$ & 28,36 & & $50.0,14.8$ & $45.3,16.2$ & \\
\hline \multicolumn{7}{|l|}{ No of AEDs } \\
\hline Mean, SD & $2.12,0.97$ & $2.24,0.84$ & & $3.1,1.1$ & $3.1,1.1$ & \\
\hline Median, IQR & $2.0,1-4$ & $2.0,1-4$ & 0.33 & $3.0,3-4$ & $3.0,2-4$ & 0.818 \\
\hline 1 & $19(33.2 \%)$ & $21(19.4 \%)$ & & $8(8.3 \%)$ & $7(7.5 \%)$ & \\
\hline 2 & $21(35.6 \%)$ & $47(43.5 \%)$ & & $13(13.5 \%)$ & $18(19.4 \%)$ & \\
\hline 3 & $14(23.7 \%)$ & $33(30.6 \%)$ & & $43(44.8 \%)$ & $33(35.5 \%)$ & \\
\hline \multirow[t]{2}{*}{4 or more } & $6(10.2 \%)$ & $7(6.8 \%)$ & & $31(32.3 \%)$ & $35(37.6 \%)$ & \\
\hline & & & 0.217 & & & 0.492 \\
\hline Physical comorbidity & $21(35.6 \%)$ & $53(49.1 \%)$ & 0.078 & $72(75.0 \%)$ & $75(80.6 \%)$ & 0.351 \\
\hline $\begin{array}{l}\text { On anti-psychotic } \\
\text { medication }\end{array}$ & $10(16.9 \%)$ & $19(17.6 \%)$ & 0.879 & $34(35.4 \%)$ & $20(21.5 \%)$ & 0.034 \\
\hline $\begin{array}{l}\text { On psychotropic } \\
\text { medication }\end{array}$ & $11(18.6 \%)$ & $14(13.0 \%)$ & 0.349 & $26(27.1 \%)$ & $18(19.4 \%)$ & 0.209 \\
\hline ASD/PDD & $18(30.5 \%)$ & $31(28.7 \%)$ & 0.859 & $24(25.0 \%)$ & $14(15.1 \%)$ & 0.088 \\
\hline $\begin{array}{l}\text { Mental \& behavioural } \\
\text { disorders }\end{array}$ & $14(23.7 \%)$ & 17 (15.7\%) & 0.224 & $8(8.3 \%)$ & $3(3.2 \%)$ & 0.134 \\
\hline Genetic syndrome & $4(6.8 \%)$ & $24(22.2 \%)$ & 0.01 & $18(18.8 \%)$ & $25(26.9 \%)$ & 0.182 \\
\hline
\end{tabular}

AEDs = anti-epileptic drugs; ID = intellectual disability; ASD = autism spectrum disorder; PDD = psychological disorders. 
Table 5.2: Comparison of the prescribed dosages of commonly used AEDs in the UK and the Netherlands cohorts.

\begin{tabular}{lcccccccc}
\hline & \multicolumn{4}{c}{ UK } & \multicolumn{4}{c}{ The Netherlands } \\
\cline { 2 - 9 } & $\mathbf{n}(\%)$ & \multicolumn{3}{c}{ Mean (mgs) SD (mgs) } & Min-max (mgs) & n (\%) & Mean (mgs) SD (mgs) Min-max (mgs) \\
\hline Valproate & $79(44 \%)$ & 1551.5 & 537.3 & $200-3000$ & $95(50.3 \%)$ & 1409.9 & 501.1 & $320-2750$ \\
Carbamazepine & $32(18 \%)$ & 725.9 & 290.7 & $120-1600$ & $93(49.2 \%)$ & 758.5 & 306.0 & $100-1600$ \\
Levetiracetam & $68(37.8 \%)$ & 1743.3 & 968.7 & $125-4000$ & $30(15.9 \%)$ & 1607.5 & 955.5 & $125-3000$ \\
Clobazam & n/a & n/a & n/a & n/a & $90(47.6 \%)$ & 21.6 & 9.8 & $5-50$ \\
\hline
\end{tabular}

AEDs = anti-epileptic drugs; $\mathrm{n} / \mathrm{a}=$ not available.

\section{Discussion}

The populations assessed probably reflect the most complex cases in Southwest England and in the Southern Netherlands. We found that a third of people with the most severe form of ID are taking three or more AEDs and this might be over twothirds in those in the Dutch cohort.

Data on the number of AEDs being prescribed in the general population is limited. While good practice suggests that monotherapy should be targeted this is quite unlikely in those with drugresistant epilepsy. Population studies in epilepsy have suggested that up to a quarter are using more than one drug. ${ }^{20,21}$ These studies have, however, not looked at specific subpopulations such as those with ID.

A retrospective study suggested that, while use of 2 concurrent AEDs provided improved efficacy over monotherapy, use of three AEDs provided no further benefit over two. ${ }^{22}$ This study did also not assess people with ID specifically.

Our results suggest that using two or less AEDs is challenging in the ID population especially given their refractory nature. Most of these populations are usually not candidates for curative epilepsy surgery. An unpublished audit in the Dutch site attempting to reduce AED polytherapy failed due to resistance of families and carers who did not consent to medication alterations.

The most frequently prescribed drug in both centres is valproate. The English centre favors the use of the non-enzyme inducing levetiracetam while in the Dutch centre carbamazepine has the preferences. This may partly be due to historical reasons as many have been taking carbamazepine for many years and this presenting difficulties in withdrawing as identified in the Dutch audit. In addition, concerns of potential behavioral side effects to levetiracetam ${ }^{23}$ may also have played a role.

Levetiracetam doses in the Dutch sample are lower than the English sample possibly reflecting the fear of behavioral side effects. ${ }^{24}$ As the English service is 
primarily psychiatric led it might reflect the confidence in identifying and managing potential psychological side effects.

A substantial number of people had VNS implanted but this seems to have made no difference to in the number of AEDs pre and post insertion. This is in line with a previous report showing that the AED treatment regime is usually not changed after VNS implantation. ${ }^{25}$

The number on anti-psychotic and psychotropic medication was higher in the Dutch sample but associated with a lower frequency of behavioral disorders. A possible explanation is that those included in the Dutch cohort had also participated in the neuropsychological screening program aimed to provide targeted psychological interventions. Evidence suggests psychotropic drugs are prescribed to over half of people with epilepsy, autism and severe/profound ID. ${ }^{13}$ In both countries there are national initiatives to reduce psychotropic prescribing for people with ID. The more modest levels of prescribing psychotropics in both centres could provide good practice templates.

ASD in the study samples were constant irrespective of the degree of ID. This is in disagreement with previous reports that ASD increases with degree of ID. ${ }^{26}$ It could be argued ASD is more difficult to diagnose with moderate to profound ID due to difficulties in testing this population.

Consistent with previous reports, there was an association between genetic syndromes with increased levels of ID. This finding is in line with the current assumption that mild forms of ID are mostly representing the lower end of the normal IQ distribution and are commonly the result of the combined contribution of genetic and environmental factors. ${ }^{27}$ Clinicians should consider genetic testing to maximize the effectiveness of their management.

We believe that epilepsy in people with ID is not just a standalone disease but part of a neurodevelopmental syndrome in many ways. The increased links with genetic aberrations further underpins this view. The joint project suggests that AEDs are only partly effective and given the increased total drug load due consideration needs to be given about long term benefits of prescribing AEDs versus people's quality of life and potential side effects. This is obviously a new dimension poorly scoped till date but requires informed debate.

\subsection{Limitations}

This pragmatic real world study is not without major limitations. The study lacks definitive comparable samples, there is a lack of seizure details, no seizure outcome data and no historical data on drug use and no attempt has been made to validate 
psychiatric diagnosis. It attempts to pool data of two populations of two countries principally identified by their nature and degree of their ID. There has been no attempt to compare seizure frequency and intensity as research in this population has shown that definitive diagnosis is difficult. Further the level of seizure control has not been looked and could be a possible confounder to the prescribing. This was a cross sectional explorative study looking at real world practice. Further limitations include the study being retrospective, involving data collection from clinical records, the variation in settings, clinician attributes and practice standards. While limitations in diagnosis and classification have been minimised by using consistent recognised diagnostic standards and rationale (Appendix 5.1) there will be inter reliability issues given the complex nature of the individuals.

\subsection{Implications for clinical practice}

We looked at a large population of people with ID and epilepsy to describe their characteristics and management issues. Our results are at variation from those for the general population and suggests while the epilepsy needs of people with ID is significant there is a need to reconsider the role of pharmacological treatment in seizure management. Possibly a larger holistic, person centred physical, mental, behavioral and social care approach might pay better dividends than just an attempt to reduce seizures with medication.

\subsection{Implications for policy}

The benefits of collecting such a wide spectrum of data include the possible generalisability of the results to routine clinical practice for people with such complex needs. Our findings could provide helpful guidance for benchmarking services and awareness of good practice. For most people with ID and epilepsy, providing care is complex requiring dedicated services which can review and audit treatment, deliver multidisciplinary care and provide genetic services in line with proposed international frameworks. ${ }^{28}$ If achieved, experience has shown that care for people with epilepsy and ID can be a 'tale with a happy ending!' 


\section{Appendix 5.1}

\section{Rationale of combining the mild-moderate and severe-profound ID and} having 2 groups

1. Each of the 2 sub-groups of severe and profound ID have a low prevalence ( $4 \%$ severe ID, and about $2 \%$ profound) and together they would combine to form $6 \%$ of the total ID population. ${ }^{29}$ Taken individually it would be difficult to achieve satisfactory power to deliver meaningful conclusions.

2. Severe and profound ID is difficult to assess and classify which causes significant issues with accuracy of specific diagnosis of severe or profound ID.

3. Epilepsy possibly due to disturbed brain function is present in $30-50 \%$ of the severe to Profound ID group as compared to $8-12 \%$ in the mild- moderate ID population and $0.6-1 \%$ in general population. ${ }^{30-32}$. 


\section{References}

1. World Health Organization. The ICD-10 classification of mental and behavioural disorders: clinical descriptions and diagnostic guidelines. Geneva: WHO Document Production Services;1992.

2. Hara, H. Autism and epilepsy: A retrospective follow-up study. Brain \& Development2007;29:486-490.

3. McGrother CW, Bhaumik S, Thorp CF, et al. Epilepsy in adults with intellectual disabilities: prevalence, associations and service implications. Seizure2006;15:376-386.

4. Forsgren L, Edvinsson S-O, Blomquist HK, et al.Epilepsy in a population of mentally retarded children and adults. Epilepsy Res1990;6:234-8.

5. Bowley C, Kerr M. Epilepsy and intellectual disability. J Intellect Disabil Res2000;44:529-43.

6. Lhatoo SD, Sander JW. The epidemiology of epilepsy and learning disability. Epilepsia2001;42(Suppl. 1):6-9.

7. Robertson J, Hatton C, Emerson E, et al. Prevalence of epilepsy among people with intellectual disabilities: a systematic review. Seizure2015; 29:46-62.

8. Robertson J, Baines S, Emerson E, et al. Service Responses to People with Intellectual Disabilities and Epilepsy: A Systematic Review. J Appl Res Intellect Disabil.2017;30:1-32.

9. Glover, G, Evison F. Hospital admissions that should not happen. Improving health and lives:ILearning disabilities observatory, 2013 (Available from: https://www.improvinghealthandlives.org.uk/ publications/1169) accessed on 28th July 2018

10. Balogh $\mathrm{R}$, Brownell $\mathrm{M}$, Ouellette-Kuntz $\mathrm{H}$, et al. Hospitalization rates for ambulatory care sensitive conditions for persons with and without an intellectual disability -a population perspective. J Intellect Disabil Res2010;54:820-832

11. Tuchman R, Rapin I. Epilepsy in autism. Lancet Neurology2002;1:352-8.

12. Berg A, Plioplys S. Epilepsy and autism: is there a special relationship? Epilepsy \& Behav2012;23:193-8.

13. Alexander R, Shankar R, Cooper SA, et al. Antipsychotic prescribing in people with learning disability: challenges and pitfalls. Br J Gen Pract2017;67:372-373.

14. Barrett A, Burke H, Cronin H, et al. Fifty plus in Ireland 2011: First results from The Irish Longitudinal Study on Ageing (TILDA). Dublin: Trinity College Dublin, 2011.

15. Doran Z, Shankar R, Keezer MR, et al. Managing anti-epileptic drug treatment in adult patients with intellectual disability: a serious conundrum. Eur J Neurol2016;23:1152-1157.

16. Kwan P, Arzimanoglou A, Berg AT, et al. Definition of drug resistant epilepsy: Consensus proposal by the ad hoc Task Force of the ILAE Commission on Therapeutic Strategies. Epilepsia2010;51:10691077.

17. Shankar R, Olotu V, Axby S, et al. Managing clients placed far away from their communities Learning Disability Practice2015;18:1;29-34.

18. Royal College of Psychiatrists. College Report 203 Management of epilepsy in adults with intellectual disability 2017 [cited 2018 July 29]. Available from: http://www.rcpsych.ac.uk/usefulresources/ publications/collegereports/cr/cr203.aspx.

19. Gottmer-Welschen LMC, Chatrou M, de Louw AJA, et al. Kempenhaeghe academic center for epileptology and residential epilepsy care, Heeze, Netherlands. Epilepsy Behav2017; 76S:S30-S34.

20. Hamer HM, Dodel R, Strzelczyk A, et al. Prevalence, utilization, and costs of antiepileptic drugs for epilepsy in Germany-a nationwide population-based study in children and adults. J Neurol2012;259:2376-84.

21. Nicholas JM, Ridsdale L, Richardson MP, et al. Trends in antiepileptic drug utilisation in UK primary care 1993-2008: Cohort study using the General Practice Research Database. Seizure2012;21:466470.

22. Poolos NP, Warner LN, Humphreys SZ, et al. Comparative efficacy of combination drug therapy in refractory epilepsy. Neurology2012;78:62-8.

23. Mula M, Trimble MR, Sander JW. Psychiatric adverse events in patients with epilepsy and learning disabilities taking levetiracetam. Seizure2004;13:55-7.

24. Chen B, Choi H, Hirsch LJ, et al. Psychiatric and behavioral side effects of antiepileptic drugs in adults with epilepsy Epilepsy Behav2017;76:24-31. 
25. Sourbron J, Klinkenberg S, Kessels A, et al. Vagus Nerve Stimulation in children: A focus on intellectual disability Eur J Paediatr Neurol2017;21:427-440.

26. Matson JL, Shoemaker M. Intellectual disability and its relationship to autism spectrum disorders Res Dev Disabil2009;30:1107-14

27. Ropers HH. Genetics of early onset cognitive impairment. Ann Rev Genomics Hum Genet. 2010;11:161-87

28. Kerr M, Linehan C, Thompson R, et al. A White Paper on the medical and social needs of people with epilepsy and intellectual disability: the Task Force on Intellectual Disabilities and Epilepsy of the International League Against Epilepsy. Epilepsia2014;55:1902-6

29. King B, Toth K, Hodapp R, et al. Intellectual Disability. In: Comprehensive Textbook of Psychiatry. 9th ed. Philadelphia: Lippincott Williams \& Wilkins. 2009 : p 444-3474.

30. Forsgren L, Beghi E, O"un A, et al. The epidemiology of epilepsy in Europe - a systematic review. Eur J Neurol2005;12:245-53.

31. Ngugi A, Bottomley C, Kleinschmidt I, et al. Estimation of the burden of active and life-time epilepsy: a meta-analytic approach. Epilepsia2010;51:883-90.

32. Linehan $C$, Kerr M, Walsh $P$, et al. Examining the prevalence of epilepsy and delivery of epilepsy care in Ireland. Epilepsia2010;51:845-52. 



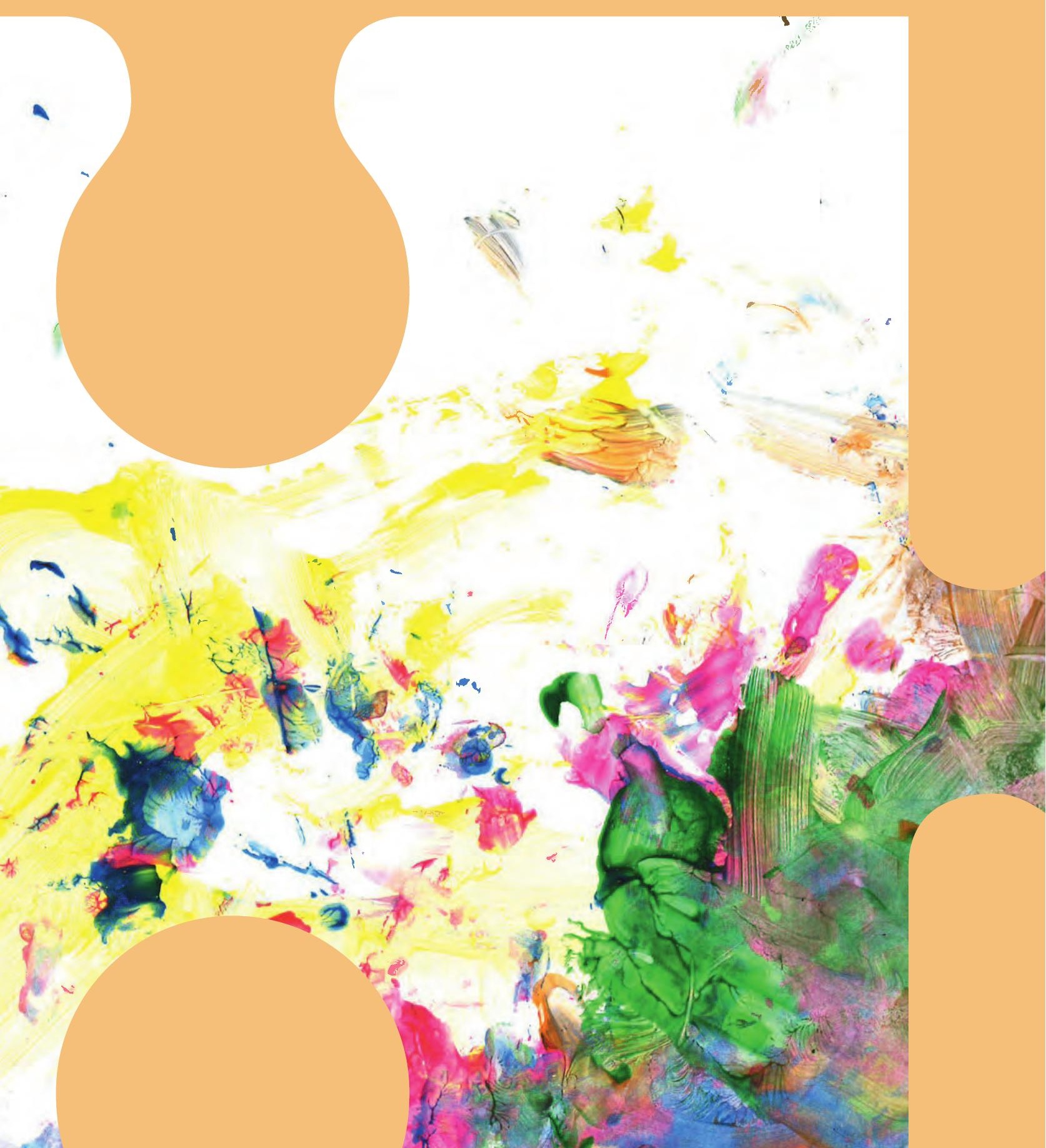




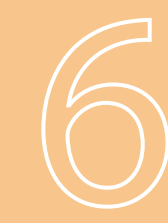

0

\section{Evaluation of Perampanel in patients with ID and Epilepsy}

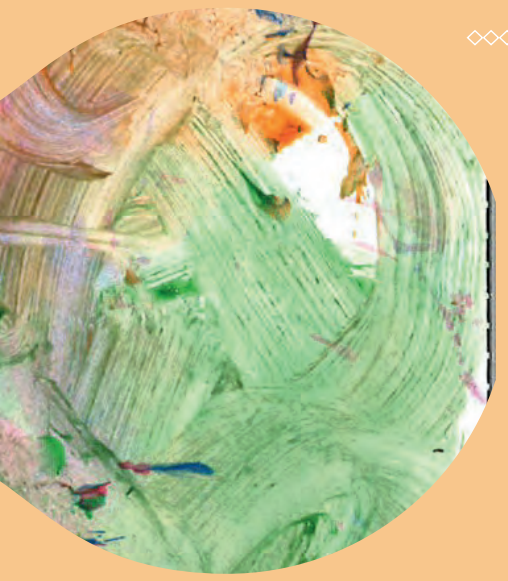

F.M. Snoeijen-Schouwenaars, J.S. van Ool, In Y. Tan,.H.J. Schelhaas, M.H.J.M. Majoie Epilepsy \& Behavior 2017;66:64-67 


\section{Abstract}

\section{Introduction}

Initial registration studies of perampanel (PMP), an AMPA receptor antagonist, have now been followed up by 'clinical' studies that confirmed its efficacy and safety in patients with refractory epilepsy. Publications on the use of PMP among patients with intellectual disability (ID) are still limited. This study extends our knowledge with respect to the relevance of PMP for patients with both ID and epilepsy, and furthermore specifies the behavioral side effects of PMP in this specific population.

\section{Methods}

Retrospective evaluation of medical records at 3, 6 and 12 months of follow-up after the initial start of PMP.

\section{Results}

62 patients were included. 21 patients (33.9\%) were female. All patients had complete data of 6 months follow-up and we were able to review 42 patients with a 1-year follow-up. Level of ID varied from borderline to profound, and mild ID was most common (43.5\%). The mean maximum daily dosage of PMP was $5.6 \mathrm{mg}$ (range 1-12 mg). Retention rates for PMP were $87.1 \%$ and $67.7 \%$ after three and six months. A trend indicated a longer mean retention time in patients with a more severe ID (borderline-mild-moderate ID: 205 days, severe-profound ID: 275 days). Seizure reduction was achieved in 53.2\%. 36 patients (58.1\%) experienced adverse effects, $80.6 \%$ of those within 3 months. $45.2 \%$ of the patients experienced somatic adverse effects. Most common were fatigue \& sleep problems, motor problems \& unsteadiness, and gastrointestinal problems. Behavioral adverse effects were present in $40.3 \%$. Most common were aggression, agitated behavior, disruptive behavior, and mood symptoms. Reasons for discontinuation of PMP were lack of efficacy in $14.8 \%$, intolerable adverse effects in $44.4 \%$, and a combination of both in $40.7 \%$.

Altogether, $24.2 \%(15 / 62)$ of the patients achieved seizure reduction without experiencing adverse effects, though none reached seizure freedom.

\section{Conclusions}

The use of PMP might lead to an effective seizure reduction without adverse effects in a minority of patients with both epilepsy and ID. Pre-existing behavioral problems or polypharmacy do not predict the occurrence of additional behavioral adverse effects, implying that these patients need not be excluded from the introduction of PMP when clinically indicated. Patients should, ideally, be monitored at a multidisciplinary clinic. 


\section{Introduction}

Epilepsy is a common health problem among people with intellectual disability (ID). The estimated prevalence of epilepsy in people with ID ranges from $15 \%$ to $30 \%$, while the prevalence of epilepsy in the general population is estimated at $0.6-1 \%{ }^{1}$

The treatment of epilepsy in patients with both epilepsy and ID might be complicated. Over the past few years, various antiepileptic drugs (AEDs) with new modes of action were introduced. Usually, patients with ID and epilepsy are excluded from the initial registration studies of these new drugs as studies with these patients are complicated for both practical and medical ethical reasons. An example of a relatively new AED is perampanel (PMP), a non-competitive $\alpha$-amino-3-hydroxy5-methyl-4-isoxazole-propionic acid (AMPA) receptor antagonist. PMP was approved in 2012 as add-on AED for partial onset seizures in patients aged twelve years or above by the European Medicines Agency (EMA). The efficacy and safety of PMP was analysed in three randomized controlled trials. ${ }^{2-4}$ The $50 \%$ responders rates were 28.5\% (PMP $4 \mathrm{mg}$ ), 35.3\% (PMP $8 \mathrm{mg}$ ), and 35.0\% (PMP $12 \mathrm{mg}$ ).

PMP was fairly well tolerated in these studies with a small number of patients suffering from generally mild side effects. The most frequently reported side effects were somnolence, fatigue, dizziness, nausea, and falls. The reported neuropsychiatric adverse effects were aggression, depression, and irritability. ${ }^{5}$

The initial registration studies of PMP have now been followed up by studies concerning clinical experience, which confirmed the efficacy and safety of PMP in patients with refractory epilepsy. ${ }^{6-9}$ However, publications on the use of PMP among patients with ID are still limited. There is one case report ${ }^{10}$ and one recent study from Shah ${ }^{11}$ that included a subgroup of patients with "learning disabilities" that was not further specified. Still, efficacy might be different in patients with both epilepsy and ID as this group also includes the often highly resistant 'epileptic encephalopathies'. Furthermore, adverse effects might be more pronounced or, conversely, might be inconspicuous, due to the fact that their presentation is influenced by polypharmacy and extensive co-morbidity. These co-morbidities in patients with both epilepsy and ID include behavioral problems with an estimated prevalence between $10 \%$ and $20 \%{ }^{12,13}$ This is even more relevant as behavioral side effects have now been recognized as one of the potential most serious side effects of PMP in the population of patients with epilepsy without ID. In order to extend our knowledge with respect to the relevance of PMP for patients with both ID and epilepsy, we studied the effect of 
this AED in patients with ID at our institute. Furthermore, we sought to specify the behavioral side effects of PMP in this specific population.

\section{Materials and methods}

\subsection{Subjects}

All patients with both epilepsy and ID, who were treated with PMP between July 2014 and January 2016, living at or visiting the outpatient clinic of Kempenhaeghe, a tertiary epilepsy centre in The Netherlands, were included in this study.

\subsection{Data collection}

The required information for the study was retrospectively retrieved from patient charts by a research student (SF) with a double check by two of the authors (JvO, FS). The baseline data included demographics (age, sex, ethnicity), level of intellectual disability according to the ICD-10 classification ${ }^{14}$, epilepsy type, age at onset of epilepsy, and previous and concomitant AEDs. PMP dosage, the duration of PMP treatment, the reasons for discontinuation of PMP, seizure frequency, and adverse effects were also assessed after a follow-up of three, six, and twelve months. The outcome with respect to seizure frequency was purely descriptive: a decrease in seizure frequency (seizure reduction), no change, or an increase in seizure frequency. Whether PMP caused a reduction or not was based on the documented (written) clinical judgment of the consultant neurologist. To identify changes in seizure frequency, we used a time-frame of 1 month of clinical data before each evaluation (baseline, 3 months, 6 months, and 12 months).

The information was obtained by case study. There was no standard questionnaire. This study was approved by the local medical ethical committee. Due to the retrospective nature and the anonymous analyses of the data, formal consent was not required. However, each included patient gave a general consent to future use of their data for research purposes at their admission to Kempenhaeghe.

\section{Results}

Demographic and clinical characteristics are shown in Table 6.1. A total of sixty-two patients treated with PMP were included, of whom 21 (33.9\%) were female. All patients had complete data of six months follow-up after the start of PMP and we 
were able to review 42 patients with a 1-year follow-up. The mean age was 27.4 years (3.2-66.8 years). The mean age at onset of epilepsy was 6.0 years (range $0-26$ years). Level of ID varied from borderline to profound, and mild ID was most common among patients (43.5\%). 46 subjects $(74.2 \%)$ were diagnosed with localization-related epilepsy and sixteen (25.8\%) with generalized epilepsy. The average number of AEDs used by patients prior to this study was four (range 0-11). The polypharmacy at the start of the study was impressive with, apart from PMP, an average of three (range 1-6) AEDs. The most commonly used concomitant AEDs were valproic acid (53.2\%), clobazam (45.2\%), and lamotrigine (40.3\%). The initial PMP dose ranged from 0,5 mg (children) to $2 \mathrm{mg}$ once a day. The titration rate was guided individually by the treating neurologist. The mean maximum daily dosage PMP was $5.6 \mathrm{mg}$ (range 1-12 mg).

Table 6.1: Demographic and clinical characteristics $(\mathrm{N}=62)$.

\begin{tabular}{|c|c|}
\hline Characteristics & Values \\
\hline Male & $66.1 \%$ \\
\hline Mean age, years (SD); & 27.4 years (17.2); range $3.2-66.8$ \\
\hline Children (<18 years) & $32.3 \%$ \\
\hline Adults (>18 years) & $67.7 \%$ \\
\hline \multicolumn{2}{|l|}{ Level of ID } \\
\hline Borderline & $4.8 \%$ \\
\hline Mild & $43.5 \%$ \\
\hline Moderate & $16.1 \%$ \\
\hline Severe & $25.8 \%$ \\
\hline Profound & $9.7 \%$ \\
\hline Mean age at onset of epilepsy (SD) & 6.0 years $(6.1)$; range $0-26$ \\
\hline \multicolumn{2}{|l|}{ Epilepsy type } \\
\hline Localized & $74.2 \%$ \\
\hline Generalized & $25.8 \%$ \\
\hline History of AEDs (SD) & 3.8 (2.9); range $0-11$ \\
\hline Mean no. concomitant AEDs (SD) & 3.0 (1.0); range $1-6$ \\
\hline 1 AED & $6.5 \%$ \\
\hline 2 AEDs & $22.6 \%$ \\
\hline 3 AEDs & $43.5 \%$ \\
\hline$>3$ AEDs & $27.4 \%$ \\
\hline Length of PMP treatment, days & $6-365$ \\
\hline$<3$ months & $12.9 \%$ \\
\hline $3-5.9$ months & $19.4 \%$ \\
\hline $6-8.9$ months & $8.1 \%$ \\
\hline 9-11.9 months & $3.2 \%$ \\
\hline \multirow[t]{2}{*}{$\geq 12$ months } & $29.0 \%$ \\
\hline & ( $27.4 \%$ had lack of follow-up data after 6 months) \\
\hline
\end{tabular}

AEDs = antiepileptic drugs; ID = Intellectual Disability; PMP = Perampanel. 


\subsection{Efficacy}

At the end of the study, seizure reduction was achieved in $53.2 \%$ of the patients. The mean PMP dosage in these patients was $6.1 \mathrm{mg}$, which is significantly higher than the mean dosage in those who did not show a seizure reduction (difference $=1.0 \mathrm{mg}$; Mann-Whitney $U$ test: $p=0.045$ ). Seizure reduction varied over time with $48.4 \%$ of the patients showing seizure reduction within three months and $17.7 \%$ of the patients between three and six months. The seizure reduction was not significantly associated with the type of epilepsy or the level of ID. No patient became seizure-free for a substantial period of time with PMP as add-on treatment.

The retention rates for PMP were $87.1 \%$ and $67.7 \%$ after three and six months, respectively. There was a trend that indicated a longer retention time in patients with a more severe ID, although this did not reach statistical significance (borderline-mildmoderate ID: 205 days, severe-profound ID: 275 days, Mann-Whitney $U$ test: $p=0.062$; see Figure 6. 1).

Eight patients discontinued PMP within three months, twelve patients between three and six months, and six patients between six and twelvemonths. Reasons for discontinuation of PMP were lack of efficacy in 14.8\%, intolerable adverse effects in $44.4 \%$, and a combination of both in $40.7 \%$ of patients. Patients who discontinued PMP had a significant lower dosage of PMP, less seizure reduction, and more adverse effects (Table 6.2).

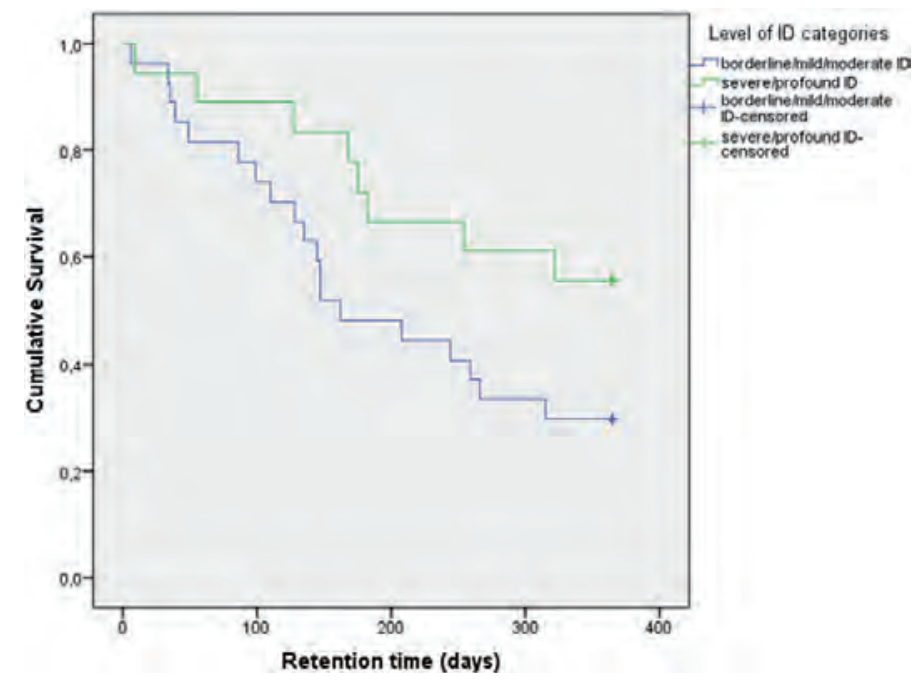

Figure 6.1: Retention on treatment with PMP in 62 patients (in days). 
Table 6.2: Characteristics of patients who continued or discontinued PMP.

\begin{tabular}{lccc}
\hline & $\begin{array}{c}\text { Continued PMP treatment } \\
\text { for at least six months }\end{array}$ & $\begin{array}{c}\text { Discontinued PMP } \\
\text { treatment }\end{array}$ & P-value \\
\hline Mean age & 28.0 & 26.0 & $0.674^{\mathrm{a}}$ \\
Level of ID & & & \\
$\quad$ Mild & $26 / 42$ & $16 / 20$ & $0.533^{\mathrm{b}}$ \\
$\quad$ Severe & $14 / 42$ & $6 / 20$ & \\
Epilepsy syndrome & & & \\
$\quad$ Localized & $33 / 42$ & $13 / 20$ & $0.254^{\mathrm{b}}$ \\
$\quad$ Generalized & $9 / 42$ & $7 / 20$ & $0.036^{\mathrm{a}}$ \\
Dosage PMP & 6.2 & 4.7 & $0.001^{\mathrm{b}}$ \\
Seizure reduction & $29 / 42$ & $5 / 20$ & $0.003^{\mathrm{b}}$ \\
Adverse effects & $19 / 42$ & $17 / 22$ & $0.030^{\mathrm{b}}$ \\
Somatic adverse effects & $15 / 42$ & $13 / 22$ & $0.029^{\mathrm{b}}$ \\
Behavioral adverse effects & $13 / 42$ & $12 / 22$ &
\end{tabular}

${ }^{\mathrm{a}}$ Mann-Whitney U test. ${ }^{\mathrm{b}}$ Chi-square test. ID $=$ Intellectual Disability.

\subsection{Adverse effects}

Thirty-six patients (58.1\%) experienced adverse effects (see Table 6.3). Most of them (80.6\%) experienced these adverse effects within three months after starting PMP. The mean PMP daily dosage in the group of patients with adverse effects was $5.3 \mathrm{mg}$ (range 1-10 mg). The presence or absence of adverse effects overall (somatic and behavioral) was not significantly related to the maximum PMP dosage (MannWhitney $U$ test: $p=0.196, p=0.954$, and $p=0.719$, respectively). Furthermore, it was not significantly associated with age, level of ID, epilepsy syndrome or seizure reduction. Adverse effects were, however, significantly associated with a lower number of concomitant AEDs ( $t=2.56, \mathrm{p}=0.013$; see Table 6.4). Adverse effects were experienced in $55.9 \%$ of subjects who achieved seizure reduction (19 out of 34 subjects), for which $42.1 \%$ (eight out of 19 subjects) discontinued PMP. This leaves 24.2\% (15/62 subjects) who achieved a seizure reduction without experiencing adverse effects, with none of the subjects becoming seizure-free. In total, 42\% (26/62 subjects) continued using PMP with no adverse effects or with adverse effects that were tolerated (and did not require the discontinuation of $\mathrm{PMP}$ ).

Overall, $45.2 \%$ of the patients experienced somatic adverse effects. The mean daily dosage of PMP in this group was $5.7 \mathrm{mg}$ (range 1.5-12 mg). Most common were fatigue \& sleep problems, motor problems \& unsteadiness, and gastrointestinal problems. More than one-third (35.5\%) of the patients experienced the adverse effects within three months and $19.4 \%$ between three and six months. One patient discontinued PMP within 10 days because of extreme somnolence that was unacceptable for the parents. 
Table 6.3: Adverse effects of PMP.

\begin{tabular}{|c|c|c|c|}
\hline & 3 months FU & 6 months FU & 12 months FU \\
\hline \multicolumn{4}{|l|}{ Somatic adverse effects } \\
\hline Fatigue and sleep problems & $14 / 62$ & $6 / 54$ & $1 / 25$ \\
\hline Motor problems and unsteadiness & $7 / 62$ & $4 / 54$ & $0 / 25$ \\
\hline Gastrointestinal problems & $7 / 62$ & $5 / 54$ & $0 / 25$ \\
\hline Urinary tract problems & $1 / 62$ & $0 / 54$ & $0 / 25$ \\
\hline Fever & $1 / 62$ & $0 / 54$ & $0 / 25$ \\
\hline Sedation & $0 / 62$ & $0 / 54$ & $1 / 25$ \\
\hline \multicolumn{4}{|l|}{ Behavioral adverse effects } \\
\hline Aggressive behavior & $7 / 62$ & $3 / 54$ & $2 / 32$ \\
\hline Agitated behavior & $7 / 62$ & $2 / 54$ & $1 / 25$ \\
\hline Disruptive behavior & $8 / 62$ & $1 / 54$ & $0 / 25$ \\
\hline Anxiety & $2 / 62$ & $0 / 54$ & $0 / 25$ \\
\hline Mood swings and depressive mood & $6 / 62$ & $2 / 54$ & $1 / 25$ \\
\hline Confusion & $0 / 62$ & $2 / 54$ & $0 / 25$ \\
\hline
\end{tabular}

$\mathrm{FU}=$ follow-up.

Behavioral adverse effects were encountered in $40.3 \%$ of patients. The mean PMP dosage in this group was $5.4 \mathrm{mg}$ (range 1-10 mg). The most common behavioral adverse effects were aggression, agitated behavior, disruptive behavior, and mood symptoms. About one-third (32.3\%) experienced these effects within three months and $11.9 \%$ between three and six months. Pre-existing behavioral problems, consisting of aggression, autism spectrum disorder, and hyperactivity disorder were prevalent in 19 patients. Ten out of the nineteen patients (52.6\%) with pre-existing behavioral problems experienced behavioral adverse effects that were more severe or different than those that already existed before the start of PMP. The presence of preexisting behavioral problems was not significantly related to the occurrence of behavioral adverse effects (Chi-square test: $p=0.452$ ). There was a trend indicating that a lower number of concomitant AEDs was associated with more behavioral adverse effects $(p=0.078)$.

Table 6.4: Characteristics of patients with and without adverse effects.

\begin{tabular}{lccc}
\hline & No side effects & Adverse effects & P-value \\
\hline Mean age & 30.6 & 25.0 & $0.332^{\mathrm{a}}$ \\
Level of ID & & & $0.340^{\mathrm{b}}$ \\
$\quad$ Mild & $15 / 26$ & $25 / 36$ & \\
$\quad$ Severe & $11 / 26$ & $11 / 36$ & $0.111^{\mathrm{b}}$ \\
Epilepsy syndrome & & & \\
$\quad$ Localized & $22 / 26$ & $24 / 36$ & $0.196^{\mathrm{a}}$ \\
$\quad$ Generalized & $4 / 26$ & $12 / 36$ & $0.701^{\mathrm{b}}$ \\
Dosage PMP & 6.3 & 5.3 & $0.013^{\mathrm{a}}$ \\
Seizure reduction & $15 / 26$ & $19 / 36$ & 2.5 \\
Mean concomitant AEDs & 3.2 & & \\
\hline
\end{tabular}

${ }^{a}$ Mann-Whitney U test. ${ }^{b}$ Chi-square test. ID = Intellectual Disability. 


\section{Discussion}

In our study on the efficacy and tolerability of PMP as add-on treatment in sixty-two patients with both epilepsy and ID, a seizure reduction in $52.3 \%$ was found. The decline in seizure frequency is in line with a pooled analysis of three, phase III studies in patients without $I^{6,7,11}$ but is less when compared to the results in the subgroup of patients with a learning disabilities. ${ }^{11}$ When looking at the patients that were still on PMP after three months, the results of our population are comparable to the results in the 'learning disability subgroup of patients' by Shah et al. However, in our study, the retention rate is clearly lower after 6 and 12 months.

None of our patients became seizure-free, which is an important point when counselling families. Seizure freedom was also rarely found in the study by Shah et al. ${ }^{11}$ who found seizure freedom in 2 out of $87(<3 \%)$ patients. In both our study and that by Shah. efficacy was less than in studies in patients without ID where seizure freedom was achieved in $5-15 \% . .^{6-9,11,15}$ Side effects were encountered in nearly $60 \%$ of our patients which is in line with both the previous studies that did not focus on patients with ID and in the study of Shah et al. who found side effects, both somatic and behavioral, in $63 \%$ of patients with learning disabilities. ${ }^{6-9,11,15}$ However, the incidence of behavioral and mood side effects (29 to 30\%) was clearly lower than our result, showing an incidence of $>40 \%$. Interestingly, in our study a lower number of concomitant AEDs was associated with higher rates of adverse effects. With respect to behavioral adverse effects, we found a trend towards statistical significance $(p=0.078)$, also pointing in the same direction.

When looking at the reasons for discontinuation, it is obvious that patients discontinued PMP due to side effects rather than to a lack of efficacy. This is comparable with the results in various previous studies on PMP. 67,9

There are several limitations with respect to the methodology of our study. Firstly, it has all the imperfections that are part of any retrospective study. In addition, the patients were treated by different neurologists and the quality of the study was highly dependent on the quality of the clinical notes that, no doubt, varied between each visit and each individual patient. In addition, as the population consisted of both patients living in the institute and patients who only attended our outpatient clinic, the quality of the feedback on efficacy and adverse effects of PMP by respectively, the educated nursing staff and the family members, will have influenced the results. We regret that the nature of our retrospective data did not allow for better quantification of drug efficacy in specific groups such as those with $<50 \%$ seizure reduction. Still, it does reflect daily clinical care. 
We conclude that the use of PMP might lead to an effective seizure reduction without adverse effects in a minority of patients with both epilepsy and ID. Adverse effects will lead to discontinuation of treatment in a substantial number of patients. Importantly, while not all patients will tolerate the addition of PMP, our data suggests that neurologists can determine this relatively quickly (mostly within the first three months of use). Intriguingly, pre-existing behavioral problems or polypharmacy do not predict the occurrence of additional behavioral adverse effects. This suggests that these patients do not have to be excluded from the introduction of PMP when presenting with a major treatment challenge. As none of our patients became seizure-free and monitoring of adverse effects in patients with both epilepsy and ID is complicated, the introduction and maintenance of PMP in these patients should, ideally, be monitored at a multidisciplinary clinic. ${ }^{16}$ 


\section{References}

1. Robertson J, Hatton C, Emerson E, Baines S. Prevalence of epilepsy among people with intellectual disabilities: a systematic review. Seizure 2015;29:46-62.

2, French JA, Krauss GL, Biton V, Squillacote D, Yang $H$, Laurenza A, et al. Adjunctive perampanel for refractory partial-onset seizures: randomized phase III study 304. Neurology 2012;79(6):589-96.

3. French JA, Krauss GL, Steinhoff BJ, Squillacote D, Yang H, Kumar D, et al. Evaluation of adjunctive perampanel in patients with refractory partial-onset seizures: results of randomized global phase III study 305. Epilepsia 2013;54(1):117-25.

4. Krauss GL, Serratosa JM, Villanueva V, Endziniene M, Hong Z, French J, et al. Randomized phase III study 306: adjunctive perampanel for refractory partial-onset seizures. Neurology 2012;78(18): 1408-15.

5. Steinhoff BJ, Ben-Menachem E, Ryvlin P, Shorvon S, Kramer L, Satlin A, et al. Efficacy and safety of adjunctive perampanel for the treatment of refractory partial seizures: a pooled analysis of three phase III studies. Epilepsia 2013;54(8):1481-9.

6. Coyle H, Clough P, Cooper P, Mohanraj R. Clinical experience with perampanel: focus on psychiatric adverse effects. Epilepsy Behav 2014;41:193-6.

7. Juhl S, Rubboli G. Perampanel as add-on treatment in refractory focal epilepsy. The Dianalund experience. Acta Neurol Scand 2016;134(5):374-7.

8. Steinhoff BJ, Bacher M, Bast T, Kornmeier R, Kurth C, Scholly J, et al. First clinical experiences with perampanel-the Kork experience in 74 patients. Epilepsia 2014;55(Suppl 1):16-8.

9. Steinhoff BJ, Hamer H, Trinka E, Schulze-Bonhage A, Bien C, Mayer T, et al. A multicenter survey of clinical experiences with perampanel in real life in Germany and Austria. Epilepsy Res 2014; 108(5):986-8.

10. Dolton E, Choudry A. Perampanel and challenging behaviour in intellectual disability and epilepsy: a management dilemma. Case Rep Psychiatry 2014;2014:409209.

11. Shah E, Reuber M, Goulding P, Flynn C, Delanty N, Kemp S. Clinical experience with adjunctive perampanel in adult patients with uncontrolled epilepsy: a UK and Ireland multicentre study. Seizure 2016;34:1-5.

12. de Winter CF, Jansen AA, Evenhuis HM. Physical conditions and challenging behaviour in people with intellectual disability: a systematic review. J Intellect Disabil Res 2011;55(7):675-98.

13. McClintock K, Hall S, Oliver C. Risk markers associated with challenging behaviours in people with intellectual disabilities: a meta-analytic study. J Intellect Disabil Res 2003;47(Pt 6):405-16.

14. World Health Organization. The ICD-10 classification of mental and Behavioural disorders: clinical descriptions and diagnostic guidelines. Geneva: World Health Organization; 1992. Available from: http://www.who.int/iris/handle/10665/37958\#sthash.k5YnXdEC.dpuf.

15. Singh K, Shah YD, Luciano D, Friedman D, Devinsky O, Kothare SV. Safety and efficacy of perampanel in children and adults with various epilepsy syndromes: a singlecenter postmarketing study. Epilepsy Behav 2016;61:41-5.

16. KerrM, Linehan C, Thompson R, Mula M, Gil-Nagal A, Zuberi SM, et al. A white paper on the medical and social needs of people with epilepsy and intellectual disability: the Task Force on Intellectual Disabilities and Epilepsy of the International League Against Epilepsy. Epilepsia 2014;55(12):1902-6. 


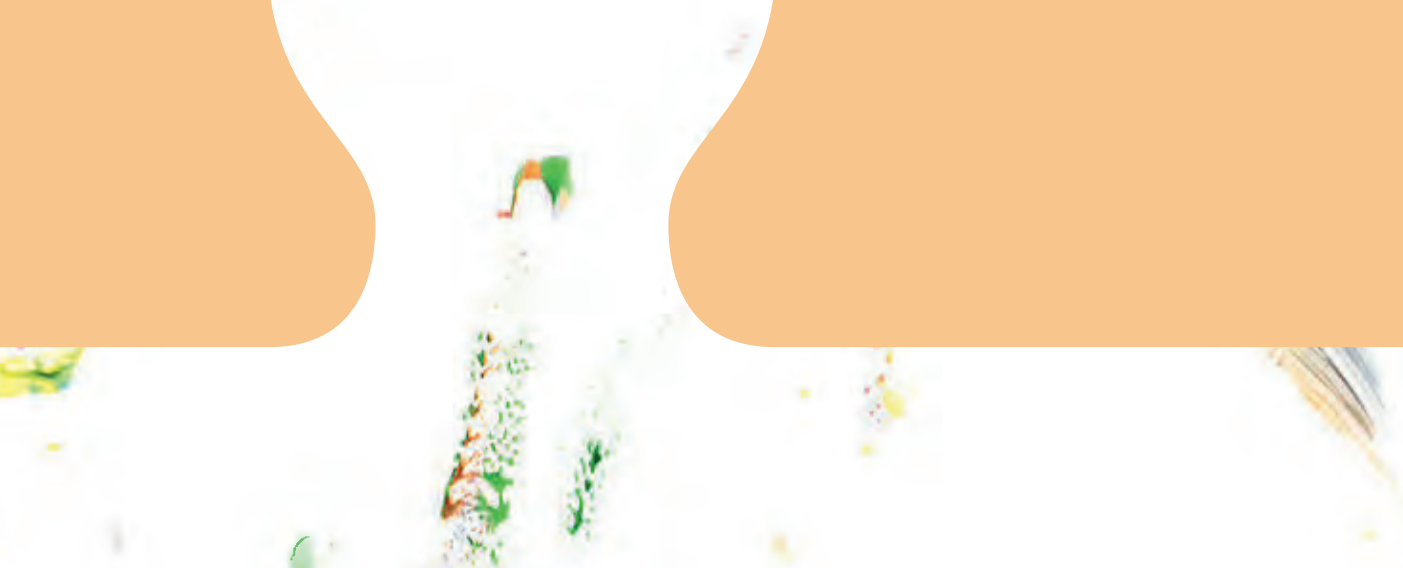

( $1 x_{i}$

wh

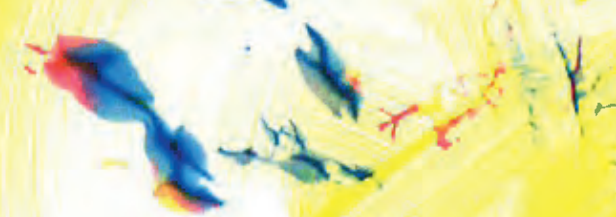

E. $x=2$.

if

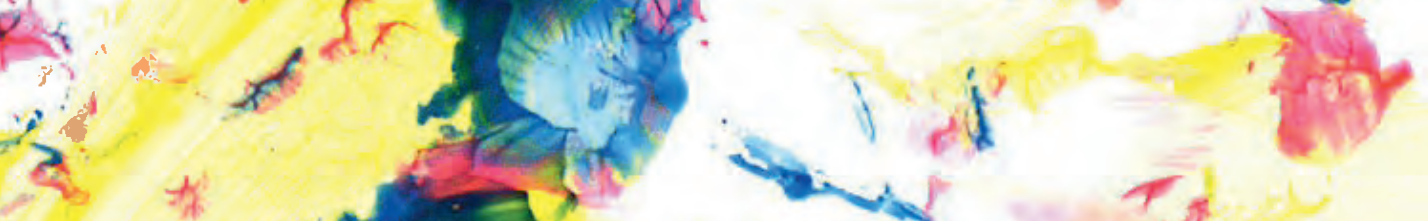

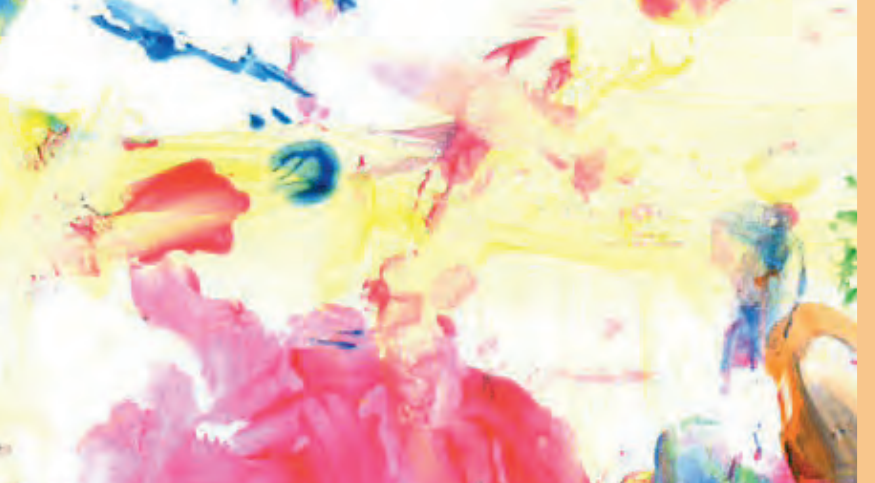




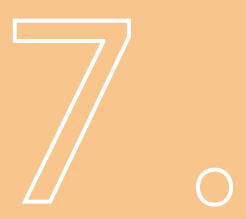

Carbamazepine and oxcarbazepine in adult patients with Dravet syndrome: Friend or foe?

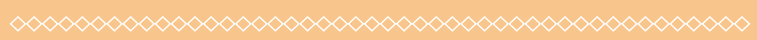

F.M. Snoeijen-Schouwenaars, M.J.B.M. Veendrick, P. van Mierlo, G. van Erp, A.J.A. de Louw, B.U. Kleine, H.J. Schelhaas, I.Y. Tan Seizure 2015;29:114-118 


\section{Abstract}

\section{Purpose}

In newly diagnosed patients with Dravet syndrome sodium channel blockers are usually avoided. However, in many adult patients the diagnosis was made long after the initiation of therapy. The purpose of our study was to acquire information concerning the potential risks and benefits of (ox)carba(ma)zepine withdrawal in adult patients with genetically confirmed Dravet syndrome.

\section{Method}

We identified 16 adults with Dravet syndrome, living in a tertiary care facility for people with epilepsy and an intellectual disability. We reviewed clinical history, genetic findings, the type and duration of sodium channels blockers that were used, seizure types and frequency, and the effect of a change in these medications.

\section{Results}

The study population consisted of 9 men and 7 women. Median age was 35 years (range 20-61 years). An attempt to withdraw carbamazepine (CBZ) was made in 9 patients. In 3 of these patients an increase in tonic-clonic seizures was observed. An attempt to withdraw oxcarbazepine (OXC) was made in 3 patients, leading to a complete stop in 2 patients. 3 of the 4 deaths in the withdrawal-group were related to epilepsy.

\section{Conclusion}

In adult patients with Dravet syndrome withdrawal of CBZ or OXC is not without risks. We suggest that (ox)carba(ma)zepine withdrawal should be considered in these patients but only if there is a good reason to do so and only if they are closely monitored. 


\section{Introduction}

Dravet syndrome is a severe epileptic encephalopathy, most frequently caused by a mutation in the SCN1A gene. It is characterized by onset of recurrent febrile and/or afebrile hemiclonic or generalized seizures in a previously healthy infant in the first 15 months of their life. This is usually followed by appearance of multiple seizure types, generally resistant to antiepileptic drugs, with developmental arrest or

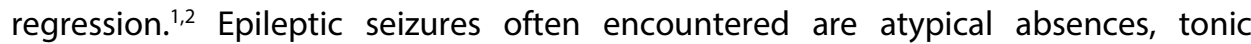
seizures, myoclonic seizures and tonic clonic seizures. The tonic clonic seizures with a focal onset persist into adulthood but with increasing age there is a reduction in the number of atypical absences and myoclonic seizures. In addition to the seizures and cognitive impairment, a progressive loss of mobility is seen partly due to cerebellar impairment. ${ }^{3}$ Prognosis is bleak with a mortality rate, also in the adult group, between 15 and $20 \% .{ }^{4}$ Most of these deaths are related to complications of a status epilepticus or due to 'sudden unexpected death in epilepsy (SUDEP)'.

There is evidence for the efficacy of stiripentol (STP), based on two randomized controlled trials. In these add-on trials STP was added to a treatment regime with valproate and clobazepam (STICLO France, STICLO Italy (not published)). Levetiracetam, topiramate and zonisamide appear to be helpful, but clinical evidence is weak (or randomized trials are not available). In addition, there are 'medication to avoid' especially concerning lamotrigine, phenytoin and carbamazepine. For lamotrigine this was carefully documented by Guerrini et al., ${ }^{5}$ They found an increase of tonic clonic and myoclonic seizures in 17 of the 21 patients with Dravet syndrome who were on lamotrigine. However, for the advice to avoid phenytoin and carbamazepine, there is less scientific evidence.

The theoretical background for a potential increase in seizure frequency by sodium channel blockers is based on the expression pattern of the SCN1A mutation. ${ }^{6}$. The SCN1A gene codes for the SCN1 alpha protein, a subunit of the voltage gated sodium channel. Voltage gated channels are the basis for every action potential, explaining their relation with epilepsy. Dravet syndrome is usually caused by 'loss of function' mutations and for this a loss of excitation would be expected. However, SCN1A mice models show a loss of excitation of the GABA-ergic interneurons without a relevant effect of the SCN1A mutation on the excitatory pyramidal neurons. ${ }^{7}$ So, in the mouse model the epilepsy is explained by a loss of inhibition of the GABA-ergic interneurons. The use of sodium channels blockers will, in this model, further reduce the already impaired inhibition. Benzodiazepines and stiripentol are assumed to be 
effective because of their GABA-ergic effect. Drugs like levetiracetam, topiramate, valproate en zonisamide have an indirect effect on GABA-ergic neurons that might explain their efficacy.

A negative effect of carbamazepine was reported in the large series of Brunklaus et al., ${ }^{8}$ where carbamazepine was the drug that was most often associated with a paradoxical effect.

Supported by these data we decided to withdraw carbamazepine in one of our adult patients with Dravet syndrome, who was on polytherapy and had frequent myoclonic seizures. The effect of carbamazepine withdrawal was a reduction in myoclonic seizures but an increase of tonic seizures. These tonic seizures evolved into a status epilepticus which was complicated by a bilateral pneumonia, due to which the patient ultimately died. Based on this experience we decided to investigate the effect of medication alteration, in particular the withdrawal of carbamazepine (CBZ) and oxcarbazepine (OXC) on seizure type and seizure frequency in adult patients with Dravet syndrome.

\section{Materials and methods}

The digital medical records of all our adult patients living in Kempenhaeghe (a tertiary care facility for people with epilepsy and an intellectual disability) with a genetically confirmed diagnosis of Dravet syndrome, were reviewed. Diagnostic criteria for Dravet syndrome as proposed by Dravet et al. ${ }^{1}$ were used in addition to the SCN1A mutation. Out-patients were excluded, as were patients with an epileptic encephalopathy not related to Dravet syndrome.

We identified 16 adult patients. Information concerning gender, age, age at onset, type of SCN1A mutation, severity of intellectual disability, and mortality were documented.

In addition, the treatment, duration of treatment with CBZ/OXC and the effect of a change in these medications on seizure type and seizure frequency were noted. Digital medical and pharmacy records, including a graphical rendering of seizure type and frequency, were available since 1992.

All available clinical and investigational information was reviewed.

Seizure types in adulthood where all classified by the neurologist in the past. In many cases video-EEG did not cover all seizure types.

Seizure frequency and type of seizures as it was documented over the years by the nursing staff was carefully studied but the conclusion whether there was a change in seizure type and frequency after the start or withdrawal of CBZ/OXC was ultimately 
based on the documented (written) clinical judgement of the consultant neurologist at the time of the anti-epileptic drug adjustment.

To identify if the seizure frequency was "increased" or "decreased", we used a time-frame of 3 months of clinical data after the last dosage reduction or complete stop. Possible related clinical factors, when documented were collected in the database.

\section{Results}

The study population consisted of 10 men and 6 women. Demographic and clinical data are summarized in Table 7.1. Median age was 35 years (range 20-61 years).

\subsection{Carbamazepine (CBZ)}

A total of eleven patients in the study-population (68.75\%) used CBZ during their lifetime. Details are presented in Table 7.2.

In nine of these patients, an attempt to withdraw CBZ was made at some stage. In six patients $C B Z$ was completely stopped. The reason to withdraw the medication varied from 'no efficacy', 'side effects' to 'based on the current literature'. In three of the nine patients (33\%), an increase in tonic clonic seizures was noted.

In three patients (case 1.4 and 9) the CBZ was replaced by either lamictal (case 1) or stiripentol (case 4 and 9), in the six other patients the CBZ was not replaced by another anti-epileptic drug after reduction or withdrawal.

In patient number 4 a possible precipitating factor of seizure aggravation was a recent fever period caused by a respiratory tract infection. In the other patients no possible related clinical factor during the reduction/withdrawal period was documented in the medical records.

\subsection{Oxcarbazepine (OXC)}

A total of five patients in the study-population $(31.25 \%)$ used OXC during their lifetime. Details are presented in Table 7.3.

In three patients an attempt to withdraw was made, in two patients a complete stop was possible. In one patient the myoclonic seizures reduced after the complete stop, in the other patient there was no change in seizure control. 


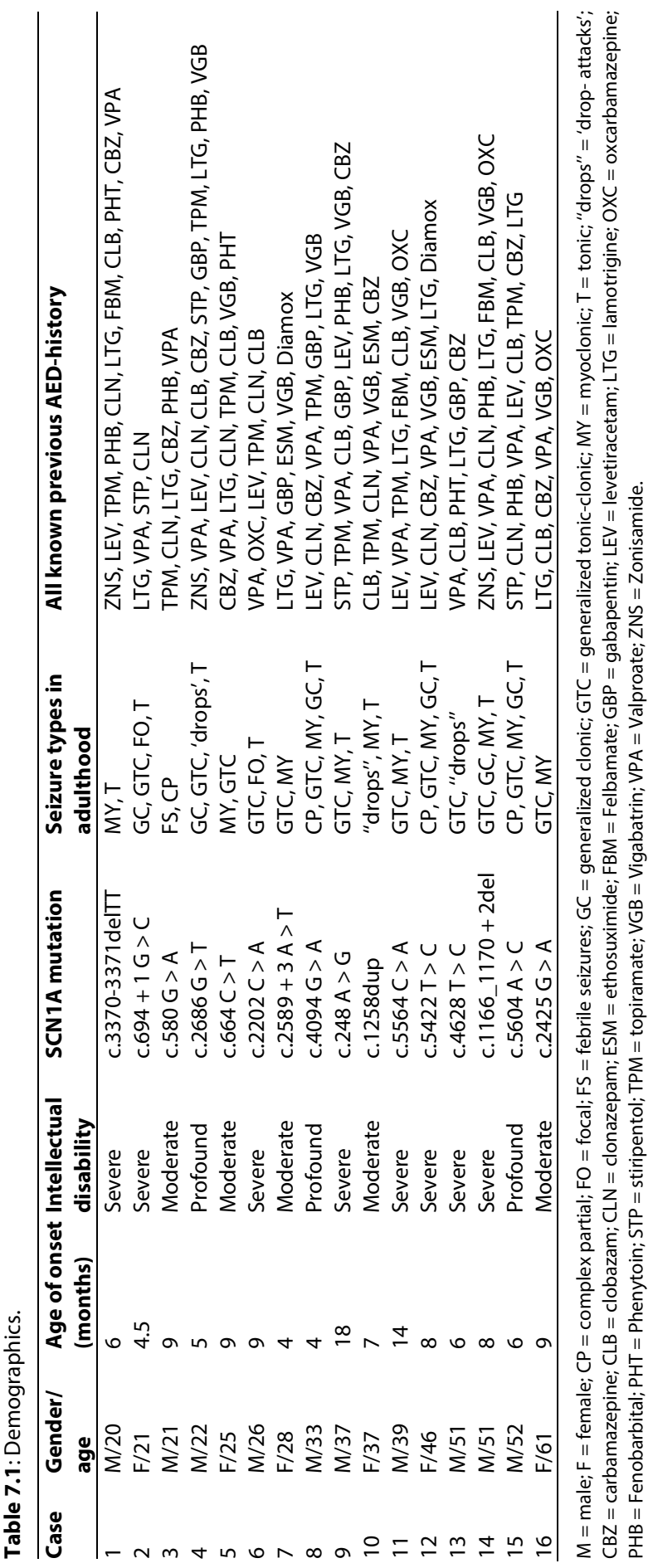




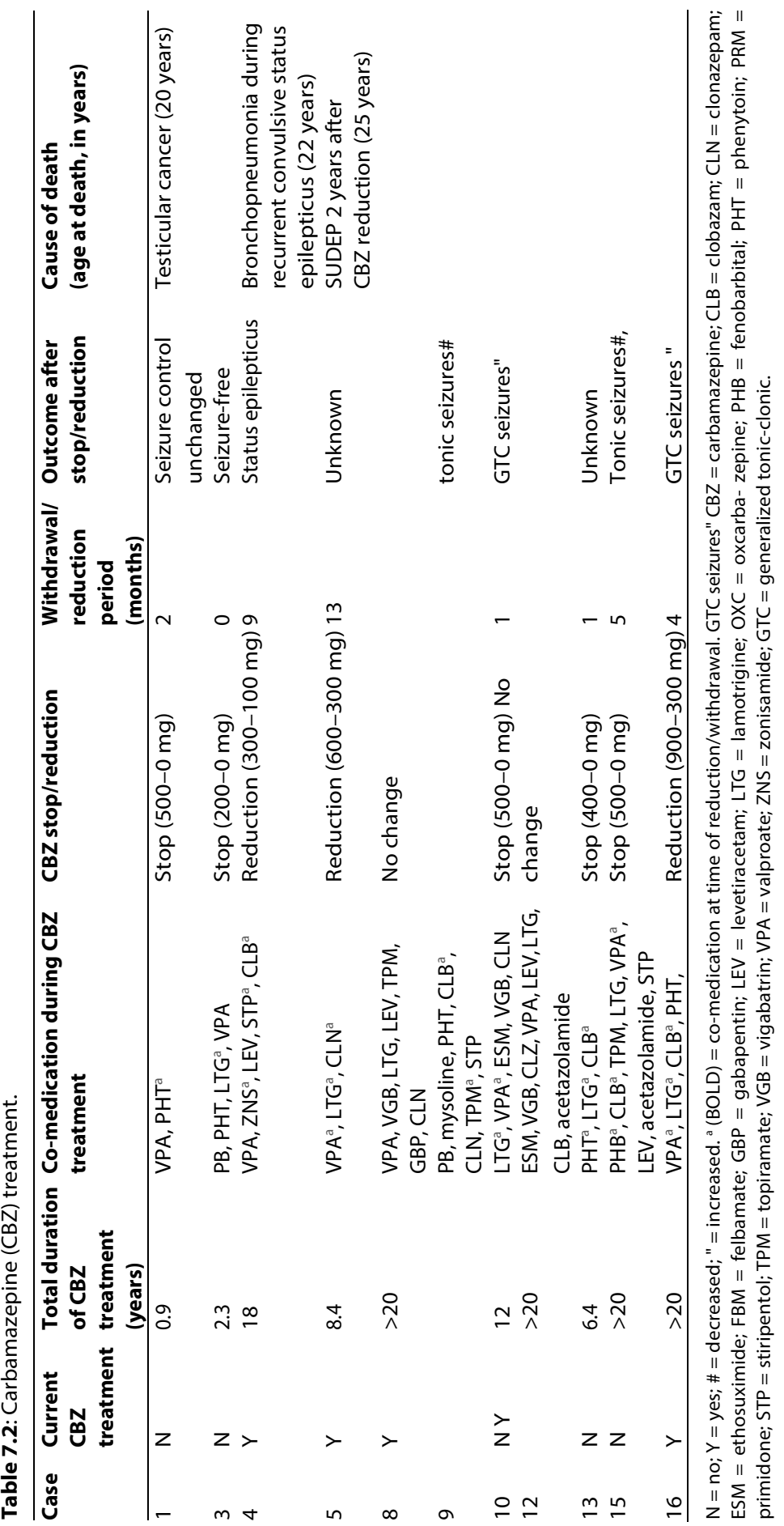




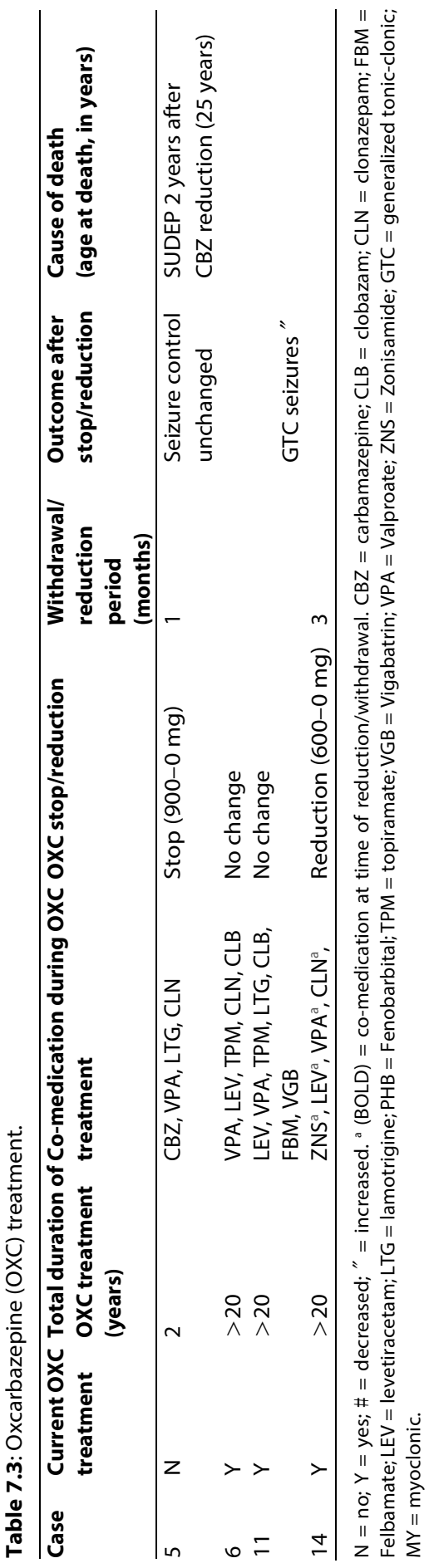


In one patient (case 5) the OXC was replaced by CBZ, in the other 2 patients (case 14 and 16) the OXC was not replaced by another anti-epileptic drug after reduction or withdrawal.

In patient number 14 a possible precipitating factor of seizure aggravation was a recurrent respiratory tract infection and feeding problems.

In the other patients no possible related clinical factor during the reduction/withdrawal period was documented in the medical records.

\subsection{Mortality}

Four patients died in total. Three of the four deaths were related to epilepsy.

In two patients the status epilepticus, either convulsive or myoclonic, was complicated by a bilateral pneumonia. Both patients had a previous status epilepticus during their lifetime.

In one of these two patients the final status epilepticus developed during the reduction of OXC.

The third patient died of SUDEP two years after the last reduction of CBZ.

\section{Discussion}

The withdrawal of carbamazepine in our institutionalized adult Dravet patients induced an increase of tonic clonic seizures in three out of nine subjects. In one patient the withdrawal of carbamazepine was, in time, related to an increase of convulsive seizures that attributed to her death. Although our experience is based on a small population the study is, in a way, unbiased as we included all our institutionalized adult SCN1A positive Dravet patients. Still, due to the retrospective nature of the study, the results should be interpreted with care.

Our retrospective data appears to be in contrast with the available, also retrospectively acquired, data from the literature. In 2011, Dravet and Guerrini ${ }^{9}$ referred to three publications that suggest a deterioration of seizure control after the start of carbamazepine. In one of these publications ${ }^{10}(\mathrm{~N}=6)$ there was a deterioration of seizure control in four patients and no effect in two subjects. In the second publication $^{11}(\mathrm{~N}=9)$ carbamazepine induced an increase of seizure frequency in two, side effects in five, and a positive effect in two patients. The third publication ${ }^{12}(\mathrm{~N}=10)$ showed a similar result. In addition to these studies, there are two relatively recent large 'clinical' Dravet studies ${ }^{8,13}$ looking at the effect of medication. In the manuscript by Brunklaus et al. ${ }^{8}(\mathrm{~N}=241)$, there were sixty patients who showed a deterioration 
after the start of medication. In thirty-six of these sixty patients $(60 \%)$ the deterioration was caused by carbamazepine. However, it was not described how often a positive or equivocal effect was seen. In the study by $\mathrm{Xu}^{13}(\mathrm{~N}=138)$, forty patients were treated with carbamazepine of whom sixteen (40\%) showed an increase in seizure frequency. There was no effect of carbamazepine in the other twenty-four patients.

There are various possible explanations for the differences in outcome between these studies and our results. First, in the previous studies, the effect of carbamazepine was studied in patients in whom carbamazepine was started, not in patients where it was discontinued. Secondly, in the literature the focus is on children whereas in our study we only included adult patients. This might be relevant as the chance of secondary lesions inducing a change in seizure type that is responsive to carbamazepine is higher in the adult group. This hypothesis is supported by the high percentage of patients with tonic seizures that was encountered in our adult patients, a type of seizure that is only rarely observed in children with Dravet syndrome or in previously studied adult patient groups. However, as was pointed out by several authors: "Some of the major convulsive seizures in adult DS have less typical aspects, for example, bilateral or asymmetric tonic posturing followed in some cases by a tonic vibratory state or clonic movements" ${ }^{4,14,15}$. For this, it is possible that the convulsive seizures that in our institute were classified as tonic, could in some cases also have been classified as 'atypical tonic clonic'. We also have to take into account that, in addition, a difference in age could be accompanied by a change in the expression of the SCN1A gene.

The expression of sodium channels is age-dependent, which explains the age of seizure onset after an initially normal development. ${ }^{6}$ In Dravet syndrome, seizure patterns change with age. In adult patients convulsive and dyscognitive seizures may become prominent. Serial interictal or ictal EEGs tend to show evidence a focal onset, while myoclonic seizures, atypical absences and photosensitivity eventually diminish or disappear in adulthood., ${ }^{4,14}$ Furthermore, in mouse models, the antiepileptic efficacy of stiripentol diminishes with age ${ }^{17}$ and combinations of drugs may be synergistic for one seizure type, but not for another. ${ }^{18}$

Important for the current concept of seizures in patients with Dravet syndrome is the selective expression of sodium channels. There are various subtypes with a different regional expression pattern that is dependent on maturation. ${ }^{6}$ It is well possible that age, chronic polytherapy or secondary neuronal damage change the expression pattern of ion channels in specific brain regions that might disrupt the balance between inhibition and excitation that will have consequences for the appropriate treatment regime. 
Even in the studies that pointed out the risk of carbamazepine on seizure frequency in patients with Dravet syndrome, there were some patients in whom the carbamazepine could not be discontinued. This was well recognized by Dravet who observed that: "In some older patient taking polytherapies, including carbamazepine, lamotrigine and phenytoin, attempt of drug withdrawal were followed by aggravation of epilepsy". ${ }^{\prime}$

The percentage of $25 \%$ patients who died in our study appears relatively high as compared to the percentage of $15-20 \%$ that is mentioned in the literature. ${ }^{4}$ This percentage might be explained by the small number of patients, the fact that in one patient the cause of death was not related to epilepsy and the severity of clinical features of our institutionalized patients. The study is too small to compare the mortality risk in those patients who have been on carbamazepine and those who have not.

We conclude that, based on the available literature, there is a relative contraindication for the use of sodium channel blockers in newly diagnosed patients with Dravet syndrome. The information concerning patients 3, 9 and 16 shows that in adult patients, the reduction of (ox)carba(ma)zepine could lead to a (partially) better seizure control. However, our data also indicate that the evidence to withdraw carbamazepine (or oxcarbazepine) is still inadequate and the withdrawal of these drugs should be very closely monitored by a neurologist that is aware of both the benefits and risks. We suggest that (ox)carba(ma)zepine withdrawal should be considered in these patients but only if there is a good reason to do so and only if they are closely monitored. 


\section{References}

1. Dravet $C$, Bureau $M$, Oguni $H$, Fukuyama $Y$, Cokar O. Severe myoclonic epilepsy in infancy (Dravet syndrome). In: Roger J, Bureau M, Dravet C, Genton P, Tassinari CA, Wolff $P$, editors. Epileptic syndromes in infancy, childhood and adolescence. 4th ed., France: John Libbey Eurotext; 2005: 89-113.

2. Depienne C, Trouillard O, Saint-Martin C, Gourfinkel-An I, Bouteiller D, Carpentier W, et al. Spectrum of SCN1A gene mutations associated with Dravet syndrome: analysis of 333 patients. J Med Genet 2009:46:183-191.

3. Rilstone JJ, Coelho FM, Minassian BA, Andrade DM. Dravet syndrome: seizure control and gait in adults with different SCN1A mutations. Epilepsia 2012;53:1421-1428.

4. Genton P, Velizarova R, Dravet C. Dravet syndrome: the long-term outcome. Epilepsia 2011;52(Suppl. 2):44-49.

5. Guerrini R, Dravet C, Genton P, Belmonte A, Kaminska A, Dulac O. Lamotrigine and seizure aggravation in severe myoclonic epilepsy. Epilepsia 1998;39:508-512.

6. Catterall WA. Sodium channels, inherited epilepsy, and antiepileptic drugs. Annu Rev Pharmacol Toxicol 2014;54:317-338.

7. Yu FH, Mantegazza M, Westenbroek RE, Robbins CA, Kalume F, Burton KA, et al. Reduced sodium current in GABAergic interneurons in a mouse model of severe myoclonic epilepsy in infancy. Nat Neurosci 2006;9:1142-1149.

8. Brunklaus A, Ellis R, Reavey E, Forbes GH, Zuberi SM. Prognostic, clinical and demographic features in SCN1A mutation-positive Dravet syndrome. Brain 2012;135:2329-2336.

9. Dravet C, Guerrini R. Dravet syndrome. Topics in epilepsy series, vol. 3. Montrouge: John Libbey Eurotext; 2011:67-81

10. Wakai $\mathrm{S}$, Ito $\mathrm{N}$, Sueoka $\mathrm{H}$, Kawamoto $\mathrm{Y}$, Hayasaka $\mathrm{H}$, Chiba S. Severe myoclonic epilepsy in infancy and carbamazepine. Eur J Pediatr 1996;155:724.

11. Horn CS, Ater SB, Hurst DL. Carbamazepine-exacerbated epilepsy in children and adolescents. Pediatr Neurol 1986;2:340-345.

12. Wang PJ, Fan PC, Lee WT, Young C, Huang CC, Shen YZ. Severe myoclonic epilepsy in infancy: evolution of electroencephalographic and clinical features. Zhonghua Min Guo Xiao Er Ke Yi Xue Hui Za Zhi 1996;37:428-432.

13. Xu X, Zhang $Y$, Sun $H$, Liu X, Yang $X$, Xiong $H$, et al. Early clinical features and diagnosis of Dravet syndrome in 138 Chinese patients with SCN1A mutations. Brain Dev 2014;36(8):676-81.

14. Akiyama M, Kobayashi $\mathrm{K}$, Yoshinaga $\mathrm{H}$, Ohtsuka $\mathrm{Y}$. A long-term follow-up study of Dravet syndrome up to adulthood. Epilepsia 2010;51:1043-1052.

15. Oguni H, Hayashai K, Awaya Y, Fukuyama Y, Osawa M. Severe myoclonic epilepsy in infants - a review based on the Tokyo Women's Medical University series of 84 cases. Brain Dev 2001;23:736-748.

16. Catarino C, Liu JYW, Liagkouras I, Gibbons VS, Labrum RW, Ellis R, et al. Dravet syndrome as epileptic encephalopathy: evidence from long-term course and neuropathology. Brain 2011;134:2982-3010.

17. Auvin S, Lecointe C, Dupuis N, Desnous B, Lebon S, Gressens P, et al. Stiripentol exhibits higher anticonvulsant properties in the immature than in the mature rat brain. Epilepsia 2013;54(December (12)):2082-2090.

18. Oakley JC, Cho AR, Cheah CS, Scheuer T, Catterall WA. Synergistic GABAenhancing therapy against seizures in a mouse model of Dravet syndrome. J Pharmacol Exp Ther 2013;345:215-224. 




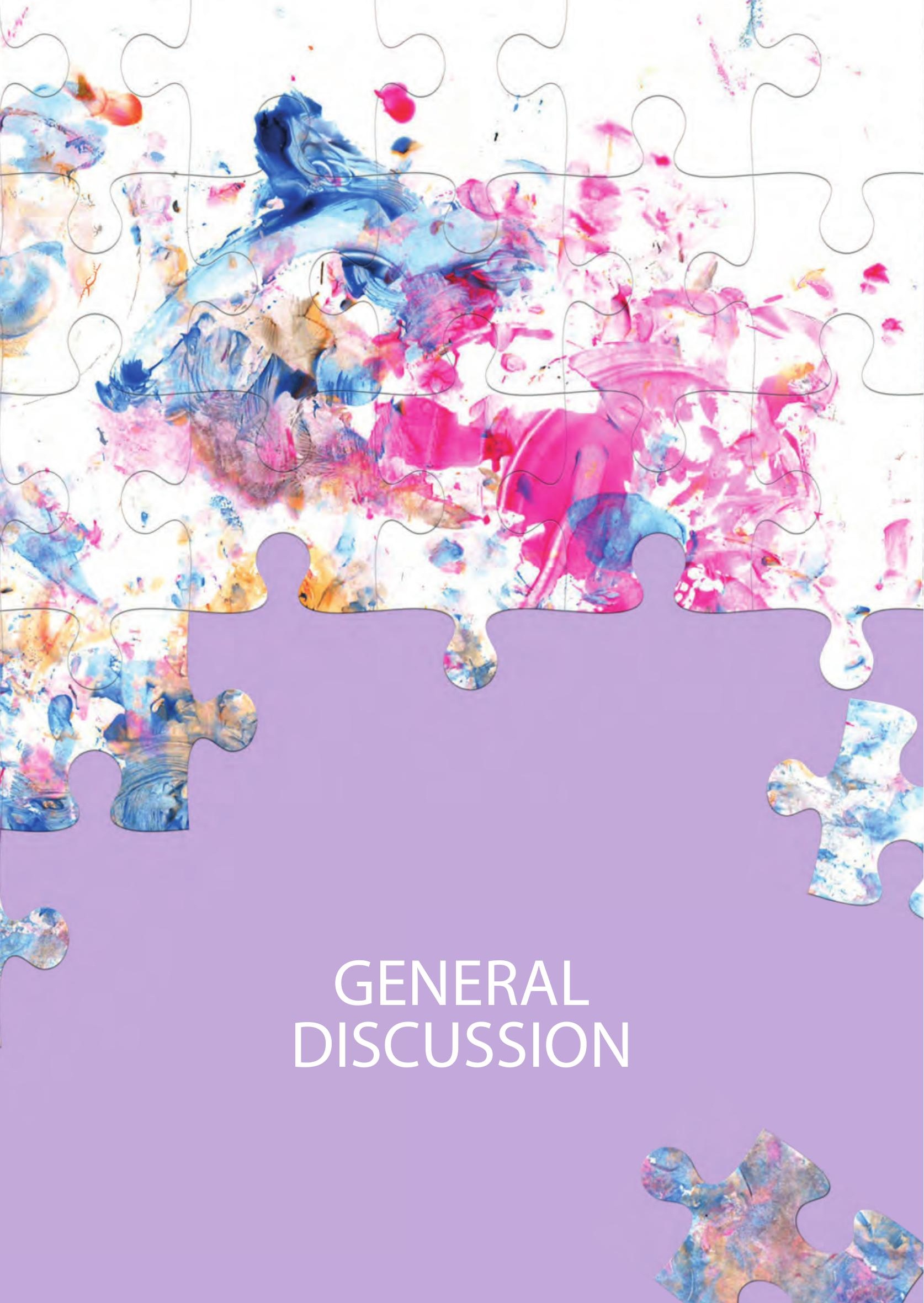




\section{General discussion}

The main motivation of the studies described in this thesis is to increase the knowledge of clinicians with regard to evidence based assessments, investigations and ultimately also treatment choices in the complex population of people with ID and epilepsy.

This chapter provides an overview of the main findings and discusses the implications for clinical practice as well as for future research.

\section{Pieces of the puzzle: diagnostic and neuropsychiatric considerations}

\section{Explanation from one perspective? - unraveling the etiology}

Although it is hardly possible for clinicians to keep up to date with all new genetic discoveries in the field of epilepsy and ID, there is increased interest in understanding ID- and epilepsy- associated (neuropsychiatric) health risks from an etiological perspective. We believe that it is important to investigate all patients who have no diagnosis. The increased interest is partly due to advanced genomic technologies now being able to identify a cause in an substantially increasing proportion of patients. Before the introduction of whole exome sequencing (WES), an etiological diagnosis could be established in about $40 \%$ of patients with previously unexplained developmental delay or ID, by using clinical examination and subsequent specific (DNA) diagnostic tests, high-resolution genome-wide chromosomal analysis, and screening for metabolic disorders.1-3

In line with the philosophy of the report 'listening for a change' that was published by the International League Against Epilepsy (ILAE) Task Force on ID and the International Bureau for Epilepsy $(\mathrm{IBE})^{4}$, in chapter 2 we evaluated the diagnostic outcomes of our multidisciplinary outpatient clinic that is dedicated to the care of patients with ID and epilepsy. We found a yield of at least $25 \%$ in patients with both intellectual disability and epilepsy with the new diagnostic technique of exome sequencing. In our study we used the strict ACMG-guidelines. ${ }^{5}$ In these guidelines the clinical information has a relatively low weight in the interpretation of variants. We identified several variants that would be classified as likely pathogenic from a clinical point of view but do not strictly fulfill the criteria for pathogenicity according to the used guidelines, for example because functional evidence is lacking. If we would add these variants to the total yield this would sum up to $30 \%$. Therefore, we expect that the application of the ACMG criteria will likely have led to an underestimation of the clinical diagnostic value of WES. 
These newly discovered diagnoses provide opportunities to delineate cause-specific characteristics and will lead to a better understanding of comorbidities and prognosis. Furthermore, establishing a genetic diagnosis may even influence treatment choices, as was the case in about $40 \%$ of the patients with a (likely) pathogenic mutation in our study. This will also be discussed in chapter 7 .

As previous reports mainly involve children, little is known about the evolution of phenotypes across their life span. In our cohort more than $50 \%$ of the patients were adults, enabling us to collect valuable data on phenotypes in the adult population. This also enables more careful counselling of family members regarding prognosis, potential natural course of the disease and life expectancy in the future. The study also contributed to the identification of potential novel pathogenic mutations. As more sequence data become available, recurrent mutations may be identified and that may help to define the phenotype of a specific mutation. For this, multidisciplinary collaboration between clinicians and more fundamental oriented laboratory specialists and scientists is of major importance.

- Genetic diagnostic investigation by WES should be considered in all patients with unexplained epilepsy and ID

- The diagnostic yield of diagnostic exome sequencing in patients with unexplained intellectual disability and epilepsy is at least $25 \%$

- The etiological diagnosis might be relevant for daily care and treatment strategy in a great proportion of the patients with a (likely) pathogenic variant

\section{Psychiatric assessment}

One of the aims of the study described in chapter 4 was to describe and evaluate a more standardized approach for performing a psychiatric assessment in people with ID and epilepsy. To date, the gold standard in psychiatric evaluation is the 'expert opinion' of the licensed psychiatrist. In our study, we set this against a standardized approach by the ID-physician. The interrater reliability between the psychiatrist's consensus and the classification by the ID-physician was strong $(\kappa=0.888)$. The initial inter-rater reliability between the psychiatrists regarding the presence of a psychiatric classification in general, was moderate $(\kappa=0.774)$, reflecting the arbitrary categorical boundaries.

This is also an important issue when trying to differentiate between primary and secondary neurodevelopmental disorders. Perhaps the structure of the new DSM-5 (symptoms, specifiers, exclusion criteria) will provide a more useful diagnostic algorithm that can reduce the heterogeneity of neurodevelopmental disorders. ${ }^{6}$ 
There are several challenges when describing psychiatric manifestations in patients with both epilepsy and ID: The mental state examination was difficult, especially in the patients with a more severe ID, because it sometimes required describing a complicated internal, subjective feeling or level of cognition. In those with a more severe level of ID, we, therefore, assessed the communicating abilities, sensory impairments, memory and ability to concentrate as being of 'greater relevance'.

Also, there was a lack in the availability of the developmental history in many adult patients, even though they had received care for decades. This lack of information made it more complicated to distinguish between behavioral disorders and, for instance, the presence of autistic spectrum characteristics.

- Psychiatric evaluation in people with ID can also be performed by a specialized IDphysician, using a broad and structured review of the patient's current problems and history.

- Developmental histories are essential for performing a psychiatric assessmentant

\section{Epilepsy and ID characteristics in relation to neuropsychiatric comorbidities}

Previous studies have shown that epilepsy and ID have each been linked to a variety of behavioral, affective, and psychiatric comorbidities. ${ }^{7-9}$ Our systematic review presented in the introduction (chapter 1) concluded that only fifteen studies were rated as of sufficient quality and they mostly focused on either the presence of ID or presence of the epilepsy in relation to neuropsychiatry. The combination of both epilepsy and ID was never systematically discussed. The studies included different types of neuropsychiatric outcomes and studied heterogeneous study populations with respect to epilepsy and ID. Despite the low level of evidence, there were a few tendencies noted:

- $\quad$ the presence of epilepsy showed a slight association with negative mood symptoms in those with ID, but not with other types of psychiatry or behavior;

- the presence of ID was linked to psychiatric disorders in people with epilepsy

- $\quad$ epilepsy-related factors indicating a more severe form of epilepsy seemed to be associated with more neuropsychiatric comorbidity in people with both epilepsy and ID.

In order to further examine the associations between epilepsy- and ID-related characteristics and neuropsychiatric comorbidities, the TRIANGLE study was conducted amongst 189 in- and outpatients who rely on the tertiary epilepsy care 
facilities of Kempenhaeghe, The Netherlands. In the dissertation of dr. J van Ool challenging behavior ${ }^{10}$ and psychogenic non-epileptic seizures (PNES) ${ }^{11}$ were taken into account as forms of neuropsychiatry.

In this thesis, we focused on mood and anxiety symptoms (chapter 3) and psychiatric comorbidity (chapter 4) as forms of neuropsychiatry.

The significant associations found in this thesis are summarized in Figure 9.1.

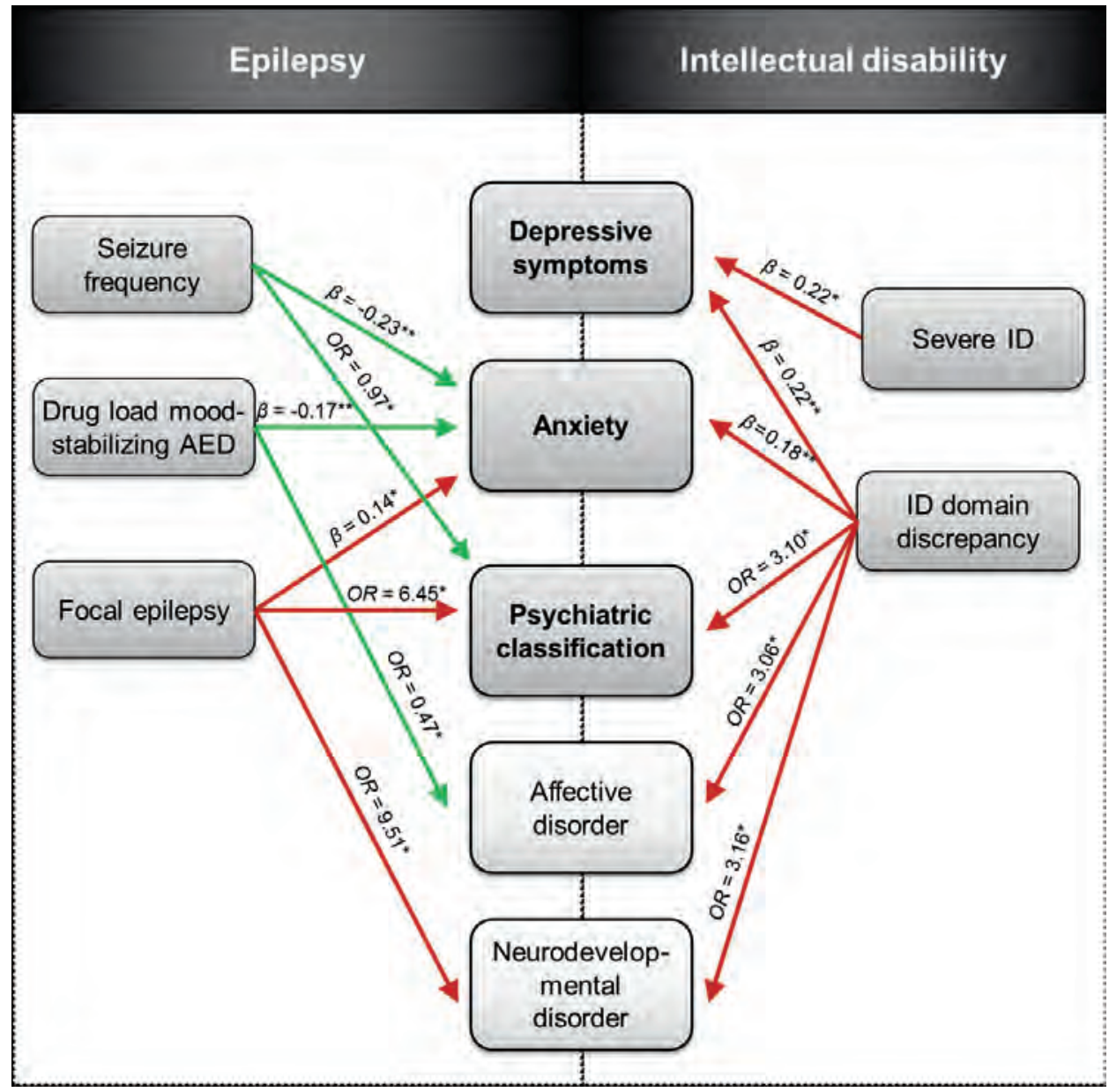

Figure 9.1: Overview of significant associations between epilepsy or ID-characteristics and neuropsychiatric comorbidities. ${ }^{*} p<0.05, * * p<0.01 . \beta=$ standardized regression coefficient. $\mathrm{OR}=$ Odds Ratio. Red lines indicate a positive association. Green lines indicate an inverse (negative) association. 
In chapter 4 we identified a psychiatric classification in $51.8 \%$ of the patients according to DSM-5. Although prevalence rates of psychiatric classifications in this population vary between different studies, this number is at the 'high-end' of the prevalence-spectrum. ${ }^{12,13}$ In chapter 3 we focused on mood and anxiety symptoms, assessed in a different way, using the Dutch version of the Anxiety, Depression and Mood Scale (ADAMS). ${ }^{14}$ The $21.7 \%$ prevalence rate of depressive symptoms is in line with the pooled prevalence of depression for patients with epilepsy in general. ${ }^{15}$ The prevalence of anxiety symptoms in our study (12.7\%) is less pronounced compared to the pooled prevalence of anxiety disorders in the general population of patients with epilepsy. The fact that a psychiatric classification (chapter 4), mood and anxiety symptoms (chapter 3) all where more often present in those with an ID discrepancy, compared to those without, stresses the relevance of the relatively new diagnostic DSM-5 aspect which underlines that in this population, all domains of ID should be taken into account, not only the 'summarizing' level of ID-classification. A higher frequency of psychiatric classification and anxiety symptoms was found among patients with a focal epilepsy. Although we did not include the supposed focus (temporal/extra temporal) of epilepsy in our analysis, the link between focal epilepsy and, for example, anxiety has also been described in previous studies ${ }^{16,17}$ and might be explained by the involvement of temporal brain structures. ${ }^{18}$ In addition, one might speculate that in those patients with a focal but extratemporal form of epilepsy, a seizure type with retained awareness might be even more frightening for patients with an ID. The psychiatric classifications associated with focal epilepsy were mostly in the neurodevelopmental spectrum (ADHD, Autism Spectrum Disorder and Intellectual disability). Intriguingly, a psychiatric classification and anxiety symptoms also appeared to be related to a lower seizure frequency, even when the use of moodstabilizing AED was taken into account. This is counter-intuitive and contrasts with a publication by Dehn et al. ${ }^{19}$ suggesting that in a group of patients without ID, a higher seizure frequency was significantly correlated with anxiety. Although we can only speculate, a possible explanation might be that most of the included patients relied on tertiary care facilities, leading to the close proximity of professional caregivers, who can provide direct care and attention. These environmental factors were beyond the scope of this study, but would be interesting to incorporate in future research.

\section{Therapeutic considerations in epilepsy and intellectual disability}

Though the mainstay of epilepsy treatment is the prescription of anti-epileptic drugs (AEDs), to date there is little evidence-based research for AED prescribing in people with ID. ${ }^{20}$ The treatment requires a person-centered approach with consideration to 
each stage of the ILAE classification of epilepsy. ${ }^{21}$ This is even more relevant to the ID population where there will be particular concerns about the potential cognitive and behavioral side effects of (new) AEDs. In this thesis we discuss the therapeutic considerations from different perspectives; by evaluating new AED-treatment options (chapter 6), we literally looked across the national borders (chapter 5) and used the etiology as important consideration (combination of chapter 2 and 7).

In our international collaboration (chapter 5), "a tale of two services" we systematically explore the AED-treatment regime in two dedicated centers for patients with ID. Our results challenge the idea that 'prescribing more than two AEDs' conflicts with good clinical practice. The most frequently prescribed drug in both centers is valproate. The English center favors the use of the non-enzyme inducing levetiracetam while in the Dutch center carbamazepine still has the preferences. This may explained by historical reasons as many have been taking carbamazepine for many years. In addition, for the specific population of Dravet syndrome withdrawing carbamazepine in adult patients who have been on this drug for many years, turned out to be more complicated than would have been expected based on the literature in children (chapter 7). It could be speculated that concerns of potential behavioral side effects to levetiracetam ${ }^{22}$ may also have played a role. Levetiracetam doses in the Dutch sample are lower than the English sample possibly reflecting the fear of behavioral side effects. ${ }^{23}$ As the English service is primarily psychiatric led it might reflect the confidence in identifying and managing potential psychological side effects.

For clinicians, it may be complicated to unravel whether the encountered complex behavior is related to the epilepsy, the AED treatment or from an epilepsy-unrelated cause. And to make it even more complex; there also needs to be consideration of the role of psychotropic medication that has epileptogenic potential. ${ }^{24,25}$

Over the past few years, various AEDs with new modes of action were introduced, but patients with ID and epilepsy are often excluded from the initial registration studies, as they are complicated for both practical and medical ethical reasons. As the epilepsy in people with ID might be highly refractory, in chapter 6 we looked at a new add-on treatment with Perampanel (PMP). Results also reflect this refractory nature, as none of our patients became seizure-free, which is an important point when counseling families. The incidence of behavioral and mood side effects was higher in our cohort than in the literature. ${ }^{26}$ But intriguingly, pre-existing behavioral problems did not predict the occurrence of additional behavioral adverse effects. Side-effects where the main reason of discontinuation, rather than the lack of efficacy. But when presented with a major treatment challenge, PMP should not be avoided as treatment option, as the use might also lead to an effective seizure reduction without adverse 
effects in a minority of patients. Monitoring of treatment- and adverse effects in this specific population should ideally be monitored at a multidisciplinary clinic, so that clinicians can detect problems relatively quickly.

Chapter 7 shows that, even when combining etiology with a personalized treatment strategy, you can face great challenges in daily clinical care of this complex population. We studied all adult patients with a known SCN1A-mutation (Dravet syndrome) living in our center. The theoretical background for potential increase in seizure frequency by sodium channel blockers (such as Carbamazepine (CBZ) and Oxcarbazepine (OXC)) in this specific group of patients is based on the expression pattern of the SCN1A mutation in GABA-ergic inhibitory neurons. Based on available literature ${ }^{27}$ CBZ and OXC should be avoided in this patients with a SCN1A mutation. In our (small) group of patients we found that in three out of nine patients a seizureincrease was observed, rather than the expected seizure-reduction. Important for this finding is that there are various subtypes of the selective regional expression pattern of sodium channels that are dependent on maturation. ${ }^{28}$ It is well possible that age, chronic polytherapy or neuronal damage changed the expression pattern of the ion channels in specific brain regions in these patients, what might disrupt the balance between inhibition and excitation that will have consequences for the appropriate treatment regime. Additionally we found that three of the four deaths in the withdrawal-group were related to epilepsy, of which one was caused by a status epilepticus during the withdrawal period. Unfortunately our study sample was too small to compare the mortality risk in those patients who have been on CBZ and those who have not. Our data do indicate the withdrawal of these drugs should be very closely monitored by a neurologist who is aware of both the benefits and the risks.

- Efficacy and adverse-effects of AEDs should be monitored in a multidisciplinary setting

- Although challenging, newer AEDs should not be avoided when facing problems in the current therapy, but the refractory nature of epilepsy should be part of counseling families

- Even when theoretically an AED should be avoided based on a specific etiology or diagnosis, withdrawal should by very closely monitored by an experienced neurologist who is aware of both the risks and benefits for this specific patient 


\section{Methodological evaluation \& limitations of the studies}

As the patients in all studies were recruited from a tertiary referral center for epilepsy, the results cannot be completely generalized to the entire population of adults with ID and epilepsy. However, given the fact that, within this population, in the studies described in chapter 3, 4 and 5, nearly eighty percent participated, and considering the limited number of studies that focus on this specific population and address the complexity of the interaction between ID, epilepsy and neuropsychiatry ${ }^{29}$, the studies provide essential new information.

Remarkably, the number of patients with an "unknown" epilepsy type is notably high in many studies in this thesis (chapter 2,3,4 and 5) This could be partly explained by the relatively high average age of the patients at the time of study in combination with the fact that most patients suffered from epilepsy from an early age and the seizure semiology might have changed in throughout the years. In addition, during the course of this thesis new diagnostic tolls became available and in some patients a genetic diagnosis was ultimately established after the publication of a specific paper.

The international study with regard to the current clinical practice in two dedicated centers for patients with ID was based on retrospective case note reviews. However the strengths of these studies being comparison of two similar services from two different countries, one of the largest ever collected ID sample population and the lack of previous evidence base prior to this papers in our view merits consideration. We believe, therefore, that this paper might help to establish a best clinical practice approach for this specific population that is not only based on current guidelines for patients without ID, but that takes the current clinical practice in both the United Kingdom and the Netherlands into account.

\section{How to proceed from here?}

\section{Clinical practice}

We tried to cluster all the relevant findings of this thesis in a graphic display. This graphic model of considerations is a continuum rather than a definitive categorical structure. It might provide a structure for clinicians to identify particular areas of concern that they may come across as part of their day-to-day work, when faced with neuropsychiatric problems experienced by people with ID and epilepsy. 


\section{FINDINGS \& IMPLICATIONS}

IN PATIENTS WITH EPILEPSY, INTELLECTUAL DISABILITY

\& NEUROPSYCHIATRIC PROBLEMS

\section{What do we know about:}

- Seizure \& epilepsy presentation

- Level of intellectual disability

- Intensity \& character of problems

\section{Etiology}

- Yield of diagnostic exome sequencing is at least 25\%

- Consideration of WES in all patients, also adults

- Might be relevant for daily care and treatment strategy

in a great portion of the patients with a (likely) pathogenic variant

\section{Be aware of:}

- Context and availability in own language

- Developmental histories are of great importance

\section{Specific factors:}

- Level of intellectual disability

- Discrepancy between ID-domains

- Presence of focal epilepsy

- (Low) seizure frequency

\section{Extra attention needed concerning medication?}

- Multidisciplinary monitoring is necessary

- Newer AEDs deserve consideration

- Never forget both the risks and benefits 


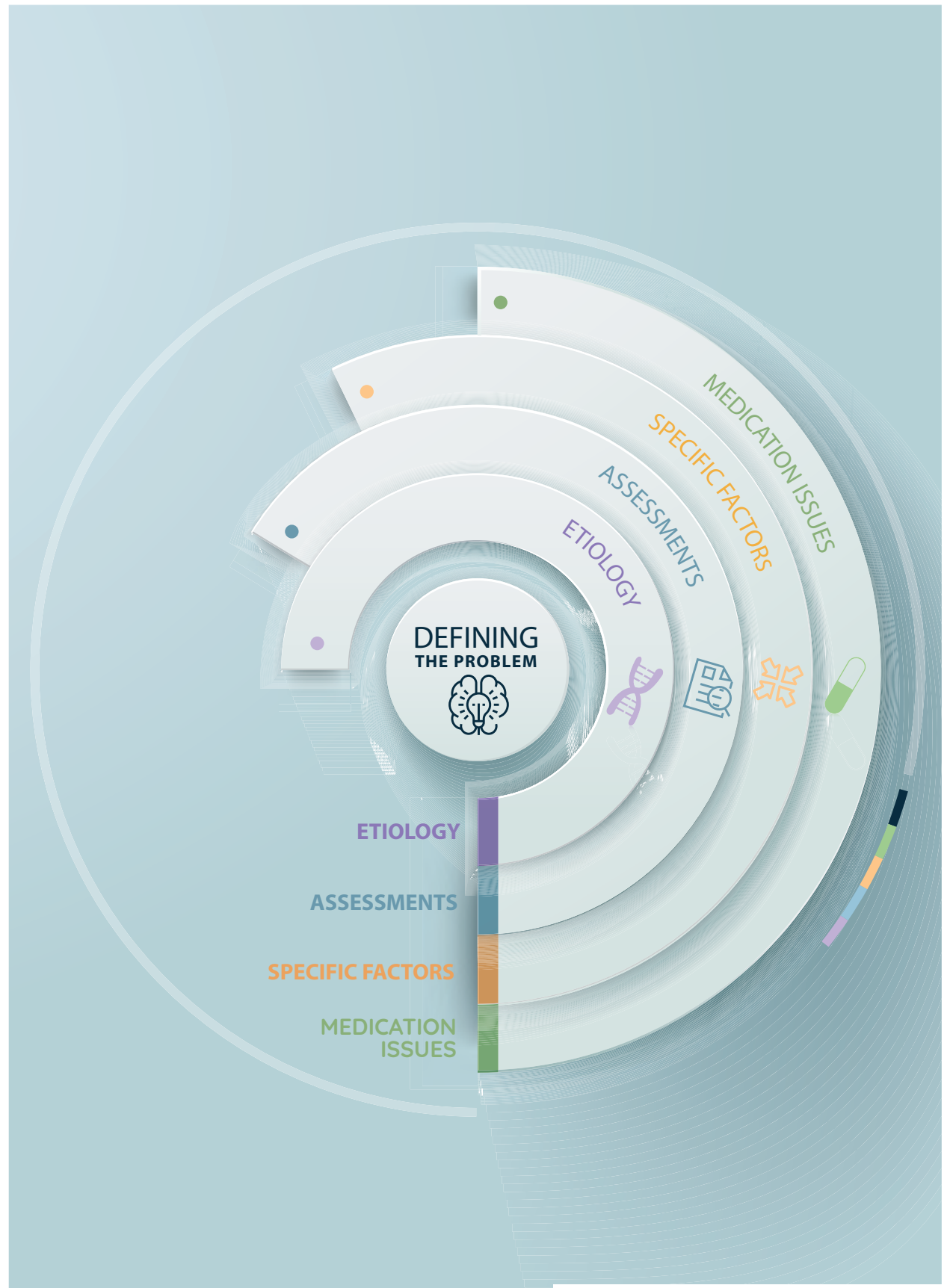

FRANCESCA SNOEIJEN - SCHOUWENAARS 
In a population in whom the combination of epilepsy and neuropsychiatric problems are common, cause and effect are often difficult to disentangle. Before any medication introduction, stop or change it is often helpful to document baseline behaviors in order to monitor changes during followup. And if a change in mood or behavior does takes place, it's important to keep in mind alternative causes other than medication alone may play a role. The behavioral changes may be the consequence of improved seizure control (which might also be a part of our findings in chapter 3) as the individual becomes more alert or responsive. Or introduction of a new drug may result in, what previously was a rapidly generalizing focal epilepsy, as only a short focal seizure, to which the individual may respond fearfully. There may even be environmental causes, such as a change in professional caregivers. But always consider the behavioral changes in the full-spectrum, in which these changes are also a possible communication of emergent sideeffects which should be explored and acknowledged.

\section{Future research}

Much work is still needed to improve the knowledge with regard to the prevalence, incidence and contributing factors of neuropsychiatric problems in people with ID and epilepsy. Fortunately, the field of research is slowly moving beyond small exploratory studies and is making more and more use of existing datasets that enhance the opportunity to compare the different experiences from international centers in order to establish a guideline that is, in line with the white paper focused $^{-}$ on this specific population. This is also the main reason we would like to expand our international collaborations with other enthusiastic researchers in the world. Ofcourse considerable challenges remain in utilizing and combining existing datasets, given differences in for instance definitions of ID and quality issues in terms of how data is gathered. Nevertheless, where there is an opportunity to also compare findings with the general population, this provides an additional window to highlight what is the same and what sometimes may be totally different for people with ID and epilepsy.

In the interaction with a patient that has a combination of ID, epilepsy and neuropsychiatric problems it is essential to unravel the detailed information with regard to etiology, level and subtype of ID, the classification of epilepsy and seizures. With regard to the classification of epilepsy and type of seizures the use of additional examinations like, for example video electroencephalography (video-EEG) would be very useful. Research in this field also is very sparse, and would be very interesting to delineate further in the coming years. There is also the potential for neuropsychiatric disturbance to occur as part of the complex epilepsy course. These symptoms may present during the peri-ictal (pre-ictal, ictal and post-ictal) or inter-ictal period. This 
was beyond the scope of our thesis, but studies in Kempenhaeghe are currently focusing on these specific events.

Current evidence suggests that most mental health problems decline with age (except for dementia). ${ }^{30}$ We have not specifically taken this decline or dementia into account in our studies, although we corrected for age in the analysis. Whether this is also true for this specific population or whether this is also an element of the epilepsy offering the opportunity to tapper the AED could be a direction for future research.

Our study in chapter 2 demonstrates that implementing WES diagnostics might be relevant for the treatment strategy; The variants of unknown significance (VUS) that we identified in novel candidate genes like in GLRA2, SAMD4A and MACF1 warrant validation in future studies, such as functional studies and studies in larger patient groups to confirm that these candidate genes are indeed associated to epilepsy and ID and further delineate the related phenotype, also at an adult age.

In general we hope to encourage young researchers to study more homogenous groups of patients, both with regard to epilepsy syndrome and degree of ID, which might help to build an evidence based structure for daily clinical practice. With this thesis we tried to make a start with gathering some prominent pieces of the puzzle. This is far from finished and we challenge other researchers in the field to help us find the remaining ones! 


\section{References}

1. Leonard $\mathrm{H}$, Wen $\mathrm{X}$. The epidemiology of mental retardation: challenges and opportunities in the new millennium. Ment Retard Dev Disabil Res Rev. 2002;8(3):117-134.

2. Ropers HH. Genetics of early onset cognitive impairment. Annu Rev Genomics Hum Genet. 2010;11:161-187.

3. Topper S, Ober C, Das S. Exome sequencing and the genetics of intellectual disability. Clin Genet. 2011;80(2):117-126.

4. Kerr M, Linehan C, Thompson R, et al. A White Paper on the medical and social needs of people with epilepsy and intellectual disability: the Task Force on Intellectual Disabilities and Epilepsy of the International League Against Epilepsy. Epilepsia. 2014;55(12):1902-1906.

5. Richards S, Aziz N, Bale S, et al. Standards and guidelines for the interpretation of sequence variants: a joint consensus recommendation of the American College of Medical Genetics and Genomics and the Association for Molecular Pathology. Genet Med. 2015;17(5):405-424.

6. Evaluation AWGoP. Practice guidelines for the psychiatric evaluation of adults. Vol Third edition 2016.

7. Hamiwka L, Jones JE, Salpekar J, Caplan R. Child psychiatry. Epilepsy Behav. 2011;22(1):38-46.

8. Aldenkamp AP, Bodde N. Behaviour, cognition and epilepsy. Acta Neurol Scand Suppl. 2005;182: $19-25$.

9. Lin JJ, Mula M, Hermann BP. Uncovering the neurobehavioural comorbidities of epilepsy over the lifespan. Lancet. 2012;380(9848):1180-1192.

10. van Ool JS, Snoeijen-Schouwenaars FM, Tan IY, Jurgen Schelhaas H, Aldenkamp AP, Hendriksen JGM. Challenging behavior in adults with epilepsy and intellectual disability: An analysis of epilepsy characteristics. Epilepsy Behav. 2018;86:72-78.

11. van Ool JS, Haenen Al, Snoeijen-Schouwenaars FM, et al. Psychogenic nonepileptic seizures in adults with epilepsy and intellectual disability: A neglected area. Seizure. 2018;59:67-71.

12. Einfeld SL, Piccinin AM, Mackinnon A, et al. Psychopathology in young people with intellectual disability. JAMA. 2006;296(16):1981-1989.

13. Cooper SA. Epidemiology of psychiatric disorders in elderly compared with younger adults with learning disabilities. Br J Psychiatry. 1997;170:375-380.

14. Hermans $\mathrm{H}$, Jelluma $\mathrm{N}$, van der Pas FH, Evenhuis HM. Feasibility, reliability and validity of the Dutch translation of the Anxiety, Depression And Mood Scale in older adults with intellectual disabilities. Res Dev Disabil. 2012;33(2):315-323.

15. Scott AJ, Sharpe L, Hunt C, Gandy M. Anxiety and depressive disorders in people with epilepsy: A meta-analysis. Epilepsia. 2017;58(6):973-982.

16. Brandt $C$, Schoendienst $M$, Trentowska $M$, et al. Prevalence of anxiety disorders in patients with refractory focal epilepsy--a prospective clinic based survey. Epilepsy Behav. 2010;17(2):259-263.

17. Kimiskidis VK, Triantafyllou NI, Kararizou E, et al. Depression and anxiety in epilepsy: the association with demographic and seizure-related variables. Ann Gen Psychiatry. 2007;6:28.

18. Jones JE, Bell B, Fine J, Rutecki P, Seidenberg M, Hermann B. A controlled prospective investigation of psychiatric comorbidity in temporal lobe epilepsy. Epilepsia. 2007;48(12):2357-2360.

19. Dehn LB, Pfäfflin $M$, Brückner $S$, et al. Relationships of depression and anxiety symptoms with seizure frequency: Results from a multicenter follow-up study. Seizure. 2017;53:103-109.

20. Doran Z, Shankar R, Keezer MR, et al. Managing anti-epileptic drug treatment in adult patients with intellectual disability: a serious conundrum. Eur J Neurol. 2016;23(7):1152-1157.

21. Fisher RS, Cross JH, French JA, et al. Operational classification of seizure types by the International League Against Epilepsy: Position Paper of the ILAE Commission for Classification and Terminology. Epilepsia. 2017;58(4):522-530.

22. Mula M, Trimble MR, Sander JW. Psychiatric adverse events in patients with epilepsy and learning disabilities taking levetiracetam. Seizure. 2004;13(1):55-57.

23. Chen B, Choi H, Hirsch $L$, et al. Psychiatric and behavioral side effecs of antiepileptic drugs in adults with epilepsy. Epilepsy Behav. 2017;76:24-31. 
24. Kerr M, Linehan C, Brandt C, et al. Behavioral disorder in people with an intellectual disability and epilepsy: A report of the Intellectual Disability Task Force of the Neuropsychiatric Commission of ILAE. Epilepsia Open. 2016;1(3-4):102-111.

25. Kerr MP, Mensah S, Besag F, et al. International consensus clinical practice statements for the treatment of neuropsychiatric conditions associated with epilepsy. Epilepsia. 2011;52(11):2133-2138.

26. Shah E, Reuber M, Goulding P, Flynn C, Delanty N, Kemp S. Clinical experience with adjunctive perampanel in adult patients with uncontrolled epilepsy: A UK and Ireland multicentre study. Seizure. 2016;34:1-5.

27. Brunklaus A, Ellis R, Reavey E, Forbes GH, Zuberi SM. Prognostic, clinical and demographic features in SCN1A mutation-positive Dravet syndrome. Brain. 2012;135(Pt 8):2329-2336.

28. Catterall WA. Sodium channels, inherited epilepsy, and antiepileptic drugs. Annu Rev Pharmacol Toxicol. 2014;54:317-338.

29. Smith KR, Matson JL. Psychopathology: differences among adults with intellectually disabled, comorbid autism spectrum disorders and epilepsy. Res Dev Disabil. 2010;31(3):743-749.

30. Evenhuis HM, Hermans H, Hilgenkamp TI, Bastiaanse LP, Echteld MA. Frailty and disability in older adults with intellectual disabilities: results from the healthy ageing and intellectual disability study. J Am Geriatr Soc. 2012;60(5):934-938. 





\section{Summary}

People with ID have a higher prevalence of epilepsy than the general population their epilepsy is more difficult to diagnose and treat, is more likely to become treatment resistant and often requires polypharmacy.

The lack of information regarding neuropsychiatric comorbidity in patients with ID and epilepsy is striking, as for instance psychiatric symptoms can have great impact on quality of life and daily functioning of a population already known for their complex needs.

In this population epilepsy is also a condition which often falls into the body-mind gap in terms of the way clinicians respond to problems. With this thesis we want to emphasize the relevance of a multidisciplinary and holistic approach, and to make a start with enhancing the evidence base of assessments, investigations and treatment choices.

\section{Part 1: Pieces of the puzzle: diagnostic and neuropsychiatric considerations}

In the first part, the studies focused on the diagnostic assessments in the field of etiology, psychiatric symptoms in general and mood $\&$ anxiety in specific.

Besides the general introduction and the main research questions, chapter 1 provides an overview of existing literature of the past 20 years by means of a systematic review, describing which neuropsychiatric comorbidities are typical for patients who have both epilepsy and ID and which factors are associated with the neuropsychiatric comorbidities in this population. Fifteen studies of sufficient quality were identified, with sometimes inconsistent results. Despite the low level of evidence, the presence of epilepsy showed a slight association with negative mood symptoms in those with ID; the presence of ID was linked to psychiatric disorders in people with epilepsy. Also, epilepsy-related factors indicating a more severe form of epilepsy seemed to be associated with more neuropsychiatric comorbidity in people with both epilepsy and ID.

One of the first considerations in clinical practice should be whether there is an etiological diagnosis or perspective that should be taken into account. Chapter 2 describes the diagnostic yield of the newest method to assess etiology, called whole exome sequencing (WES). We also discuss the potential consequences of the newly established genetic diagnosis for the treatment strategy. We found an additional yield of at least $25 \%$ in patients with both intellectual disability and epilepsy with this new technique. These newly discovered diagnoses provide opportunities to delineate cause-specific characteristics and may lead to a greater understanding of 
comorbidities and prognosis. Furthermore, establishing a genetic diagnosis may even influence treatment choices, as was the case in about $40 \%$ of the patients with a (likely) pathogenic mutation in our study.

In order to further examine the associations between epilepsy- and ID-related characteristics and neuropsychiatric comorbidities, in chapter $\mathbf{3}$ we focused on mood and anxiety symptoms and in chapter $\mathbf{4}$ the focus was on psychiatric comorbidity as forms of neuropsychiatry. Both studies were part of the TRIANGLE study, conducted amongst 189 in- and outpatients who rely on the tertiary epilepsy care facilities of Kempenhaeghe.

The assessment of symptoms was different in chapter 3, compared to chapter 4. As we first assessed mood and anxiety symptoms using the Dutch version of the Anxiety, Depression and Mood Scale (ADAMS). The $21.7 \%$ prevalence rate of depressive symptoms is in line with the pooled prevalence of depression for patients with epilepsy in general. The prevalence of anxiety symptoms in our study (12.7\%) is less pronounced compared to the pooled prevalence of anxiety disorders in the general population of patients with epilepsy. The assessment of a psychiatric classification in chapter 4 was based on a broad and structured review and observation of the patient's problems and history using DSM-5 as guidance.

Our studies show that a psychiatric classification (chapter 4), mood and anxiety symptoms (chapter 3 ) all where more often present in those with an ID discrepancy. Also a higher frequency of psychiatric classifications and anxiety symptoms was found among patients with a focal epilepsy. Intriguingly, a psychiatric classification and anxiety symptoms also appeared to be related to a lower seizure frequency, even when the use of mood-stabilizing AED was taken into account.

\section{Part 2: Therapeutic considerations in epilepsy and intellectual disability}

In the second part, we discuss the therapeutic considerations from different perspectives; we literally looked across the national borders to describe the AED use in the population of people with ID and epilepsy in chapter 5, we evaluate new AEDtreatment options in chapter $\mathbf{6}$, and used the etiology as important consideration in adjusting treatment strategies in chapter 7 .

Our results in chapter 5 suggest that using two or less AEDs is challenging in the ID population especially given their refractory nature. The most frequently prescribed drug in both UK and the Netherlands is Valproate. The English center favors the use of the non-enzyme inducing Levetiracetam while in the Dutch center Carbamazepine has the preferences. The refractory nature of the epilepsy in this population also reflects in the results of our study in chapter 6 , as none of our patients became 
seizure-free on a new add-on AED treatment with Perampanel (PMP). The incidence of behavioral and mood side effects was higher in our cohort than in other literature. Side-effects where the main reason of discontinuation, rather than the lack of efficacy. But we also found that pre-existing behavioral problems did not predict the occurrence of additional behavioral adverse effects. Monitoring of treatment- and adverse effects should ideally be monitored at a multidisciplinary clinic, so that clinicians can detect problems relatively quickly.

Chapter 7 shows that when combining etiology with a personalized treatment strategy based on available literature, you can still face challenges in daily clinical care. We studied all adult patients with a known SCN1A-mutation (Dravet syndrome) living in Kempenhaeghe. Based on available literature the recommendation is to withdraw these patients from Carbamazepine (CBZ) and Oxcarbazepine (OXC) as these AEDs are relatively contraindicated when diagnosed with this specific mutation. In our cohort we found that in three out of nine patients a seizure-increase was observed when trying to withdrawal, rather than the expected seizure-reduction. Additionally we concluded that three of the four deaths in the withdrawal-group were related to epilepsy, of which one was caused by a status epilepticus during the withdrawal period. Our data therefore indicates that the withdrawal CBZ or OXC should be closely monitored by a neurologist that is aware of both the benefits and the potential risks of changing treatment strategy in this specific patient.

Finally, chapter $\mathbf{8}$ provides a general discussion of the results of the studies and implications for future research. We tried to cluster all of the relevant findings of this thesis in a graphic display, which provides a structure for clinicians that has the option to identify particular areas of concern that they may come across as part of their dayto-day work, when faced with neuropsychiatric problems in this specific population. 



\section{Samenvatting}

In vergelijking met de reguliere bevolking komt epilepsie vaker voor bij mensen met een verstandelijke beperking (VB). Daarnaast is de epilepsie in deze groep zowel lastiger te diagnostiseren als te behandelen en is zelfs behandeling met vele verschillende soorten antiepileptica vaak onvoldoende om iemand ook daadwerkelijk aanvalsvrij te krijgen.

Het is opvallend hoe weinig er bekend is over neuropsychiatrische comorbiditeit, ondanks dat we weten dat dit een grote invloed kan hebben op het dagelijks leven en de kwaliteit van dit leven in deze reeds kwetsbare doelgroep.

In de praktijk worden deze problemen vaak in eerste instantie vanuit 1 invalshoek bekeken door artsen of overige professionals; of het medische wordt grondig onderzocht, of de psychische problemen staan voorop. Met dit proefschrift willen we benadrukken dat juist multidisciplinair werken en holistisch denken vereist is bij dit soort complexe problematiek. Daarnaast willen we een begin maken met de verbetering van evidence-based overwegingen op het gebied van diagnostiek, beeldvorming en behandelopties.

\section{Deel 1: Stukjes van de puzzel: diagnostische en neuropsychiatrische overwegingen}

In het eerste deel van dit proefschrift ligt de focus van de artikelen op diagnostische mogelijkheden op het gebied van etiologisch onderzoek, psychiatrische symptomen in het algemeen en stemming \& angst in specifiekere zin.

Hoofdstuk 1 bestaat naast de algemene introductie ook uit een literatuursamenvatting van bestaande wetenschappelijke literatuur uit de afgelopen 20 jaar. We hebben daarbij gekeken naar de samenhang tussen epilepsie, VB en neuropsychiatrische problemen. Na grondige bestudering van deze literatuur blijken er enkel vijftien studies van voldoende kwaliteit over te blijven, waarvan ook een aantal tegenstrijdige resultaten laten zien. Ondanks de lage bewijskracht, kunnen we toch voorzichtig concluderen dat er een link lijkt te zijn tussen stemmingsgerelateerde problematiek en epilepsie in de groep van mensen met VB. Daarnaast vonden we dat het hebben van een VB verband houdt met psychiatrische diagnoses in de groep van mensen met epilepsie. Tot slot lijkt er uit deze literatuursamenvatting te komen dat de ernstigere vormen van epilepsie gerelateerd kunnen zijn aan neuropsychiatrische comorbiditeit bij mensen met een VB en epilepsie. 
Wanneer men in de dagelijkse praktijk neuropsychiatrische problemen vermoed, zou een van de eerste overwegingen moeten zijn: weten we of hier een overkoepelende etiologische diagnose de verklaring voor kan zijn?

Hoofdstuk 2 beschrijft de opbrengst van de nieuwste methode van etiologisch onderzoek, genaamd Whole Exome Sequencing (WES). Daarnaast beschrijven we in dit hoofdstuk ook of de gevonden diagnoses mogelijk de epilepsiebehandeling op termijn zouden kunnen beïnvloeden. In dit onderzoek vonden we bij $25 \%$ van de mensen met VB en epilepsie alsnog een diagnose dmv WES, ondanks dat bijna iedereen in het verleden al andere onderzoeken had gehad zonder resultaat. Deze nieuw ontdekte diagnoses kunnen er aan bijdragen om in de toekomst meer duidelijkheid en overzicht te krijgen in het voorkomen van comorbiditeit en een inzicht geven in prognoses op langere termijn. We vonden dat bij $40 \%$ van mensen met een bekende diagnose, dit invloed zou kunnen hebben op de keuze van medicatie en overwegingen in het behandelplan voor de toekomst.

Om de effecten van verschillende epilepsie- en VB-variabelen op neuropsychiatrische comorbiditeit na te gaan, onderzochten we in hoofdstuk 3 stemming- en angstklachten, en in hoofdstuk $\mathbf{4}$ psychiatrische symptomen als uiting van deze 'neuropsychiatrische comorbiditeit'. Beide studies waren onderdeel van de grotere TRIANGLE studie, die werd uitgevoerd bij 189 volwassenen met epilepsie en VB die afhankelijk zijn van gespecialiseerde 24-uurs epilepsiezorg van Kempenhaeghe. De manier van diagnosticeren verschilde tussen hoofdstuk 3 en 4 . In eerste instantie werden de stemming en angstklachten $\mathrm{dmv}$ de Nederlandstalige Anxiety Depression AND Mood Scale (ADAMS) in kaart gebracht. We vonden bij 21.7\% van de mensen met epilepsie en VB een verhoogde score op depressieve kenmerken, iets wat overeenkomt met de reguliere bevolking. Het voorkomen van angst symptomen is met $12.7 \%$ echter minder uitgesproken in deze populatie dan wanneer we kijken naar mensen met alleen epilepsie. De methode om een tot een psychiatrische classificatie te komen in hoofdstuk 4 was gebaseerd op een zeer uitgebreide en gestructureerde samenvatting van de voorgeschiedenis van de patiënt, gecombineerd met gestructureerde interviews of observaties, afhankelijk van het niveau van de patiënt, gebaseerd op DSM-5.

De studies laten zien dat een psychiatrische classificatie (hoofdstuk 4) en stemming- of angstklachten (hoofdstuk 3 ) vaker aanwezig zijn wanneer er sprake is van een VB domein discrepantie. Een discrepantie is een groot verschil tussen de verschillende domeinen van VB in een en dezelfde patiënt (adaptief, cognitief of sociaal-domein). Daarnaast vonden we dat psychiatrische classificaties en angstklachten vaker voorkwamen bij mensen met een focale epilepsie. Bijzonder genoeg kwamen deze symptomen en classificaties juist minder vaak voor bij 
patiënten met een lage aanvalsfrequentie, zelfs wanneer we in de analyses corrigeerden voor het gebruik van stemmingsstabiliserende anti-epileptica (AED).

\section{Deel 2: Therapeutische overwegingen}

In het tweede deel van dit proefschrift beschrijven we enkele overwegingen op het gebied van (epilepsie)behandeling en bekijken dit vanuit verschillende invalshoeken; zo kijken we letterlijk over de grens in onze internationale studies om het gebruik van AEDs te beschrijven in hoofdstuk 5, we bespreken de plek van nieuwere medicatie in hoofdstuk 6, en gebruiken de etiologische diagnose als belangrijke onderlegger om veranderingen in een behandelplan door te voeren in hoofdstuk 7.

Onze resultaten in hoofdstuk 5 laten zien dat het rationeel gebruik van 2 of minder AED in de populatie van mensen met VB eerder uitzondering is dan regel. Het toont nog maar eens dat dit een zeer moeilijk behandelbare groep is. We vergelijken resultaten uit Nederland en het Verenigd Koninkrijk (VK), in beide landen wordt valproaat het meest voorgeschreven. Daarnaast zijn er ook een aantal verschillen in voorschrijfgedrag; zo geven de artsen in het VK de voorkeur aan het nietenzyminducerende levetiracetam, terwijl wij in NL eerder voor carbamazepine kiezen.

De refractaire epilepsie in deze doelgroep komt ook naar voren in hoofdstuk 6, waar we zien dat geen enkele patiënt aanvalsvrij wordt met het nieuw geïntroduceerde middel Perampanel (PMP). Daarnaast zagen we dat het voorkomen van bijwerkingen op het gebied van gedrag en/of stemming hoger was in onze onderzochte populatie dan in andere studies. Bijwerkingen waren dan ook de voornaamste reden voor het staken van deze medicatie. We vonden echter ook dat reeds bestaande gedragsproblemen niet verergerd werden door de start van dit nieuwe medicijn. We pleiten daarom in het artikel ook dat starten, opbouw en stoppen van deze medicatie onder multidisciplinaire begeleiding gebeurd, zodat potentiele problemen eerder herkend worden.

De bevindingen in hoofdstuk 7 laten zien dat zelfs wanneer je weet welke etiologische diagnose iemand heeft, en je behandelplan op basis van beschikbare evidence-based literatuur hierop afstemt, je alsnog problemen kunt tegenkomen in de klinische praktijk. We hebben alle volwassen patiënten beschreven met een SCN1A-mutatie (Dravet syndroom), die gebruik maakten van de 24-uurs zorg van Kempenhaeghe. Op basis van de literatuur en theorie zouden we er verstandig aan doen om in deze groep de medicamenten carbamazepine (CBZ) en oxcarbazepine (OXC) af te bouwen. In de door ons onderzochte groep vonden we echter dat tijdens de afbouw van CBZ drie van de negen patiënten juist een toename lieten zien in aanvalsfrequentie, in plaats van de verwachte afname van het aantal aanvallen. 
Daarnaast zagen we in de groep waarin op enig moment een afbouw had plaatsgevonden, dat drie van de vier sterfgevallen gerelateerd waren aan epilepsiefactoren. Een patiënt overleed tijdens een status epilepticus, tijdens afbouw.

De beschreven ervaringen in ons artikel laten zien dat er geen 'hard theoretisch bewijs' is om direct te besluiten tot afbouw wanneer je een nieuwe etiologische diagnose ontdekt. Het is van groot belang dat aanpassingen in het behandelplan op basis van onderliggende etiologie gebeuren in multidisciplinair verband onder leiding van een ervaren neuroloog, die zich goed bewust is van zowel de voordelen, nadelen en risico's van een therapeutische verandering voor deze specifieke patiënt.

Tot slot worden in hoofdstuk $\mathbf{8}$ alle resultaten uit dit proefschrift samengevat en bediscussieerd. De sterke en zwakke punten komen aan de orde en we bespreken mogelijke aanknopingspunten voor verder onderzoek in de toekomst. We hebben geprobeerd om alle klinisch relevante informatie, verkregen tijdens de uitgevoerde onderzoeken, in een grafische weergave te plaatsen. Op deze manier hopen we enige handvaten te bieden aan artsen en professionals wanneer ze tijdens hun dagelijks werk tegen neuropsychiatrische problemen aanlopen in deze specifieke doelgroep. 


\section{Valorisation}

\section{Relevance of findings}

It is not exactly clear how many people with intellectual disability (ID) live in the Netherlands. According to calculations by the Sociaal Cultureel Planbureau (SCP), in 2013 there were around 142,000 people with ID. Additionally there are approximately 1.4 million people with a borderline ID (IQ between 70 and 85). The expenditure on the care for people with ID amounted to around 7.3 billion euros in $2015,8.6 \%$ of the total healthcare costs in the Netherlands. ${ }^{1}$

People with ID have much higher rates of epilepsy than the general population $(22.2 \% \text { vs } 1 \%)^{2,3}$, their epilepsy often is more difficult to diagnose and treat, it is more likely that treatment requires polypharmacy and patients may find it harder to communicatie their wishes and needs. ${ }^{4}$

People with ID and epilepsy are part of a vulnerable population in society, whom deserves the best healthcare. But care and treatment pathways for this complex subpopulation are poorly defined and there are no strict specific national or international guidelines available yet.

Epilepsy and ID have been linked to a variety of behavioral, affective and psychiatric comorbidities ${ }^{5-7}$, but we also know that epilepsy a condition which also often falls into the mind-body gap in terms of the way clinicians or services respond ${ }^{8}$. This is one of the motivations to carry out this PhD in a "twin"-form, that stresses the relevance of a multidisciplinary and holistic approach. This thesis covers the medical perspectives, the other thesis is performed by a behavioral scientist.

These theses are an effort to make a pragmatic, yet evidence-based, bridge over the yawning gap of available literature and challenges faced in daily clinical practice.

In the first part of this thesis we showed that unraveling the etiological diagnosis could be beneficial to both adult as well as pediatric patients, and should be considered at any age and any patient. By using whole exome sequencing (WES) we were able to find an explanation in more than $25 \%$ of patients, and in over $50 \%$ of these patients this diagnosis might even be relevant for treatment strategy in the future.

In a population in whom the combination of epilepsy and neuropsychiatric problems are common, cause and effect are often difficult to disentangle, but our thesis reveals that the role of epilepsy characteristics seems to be modest compared to ID-characteristics. This shows that there should always be attention for other factors besides epilepsy-related problems. 
Before any medication introduction, stop or change it would be helpful to document baseline behaviors in a more standardized way, in order to monitor changes during follow-up. Publishing our evaluation of such an approach to psychiatric symptoms would therefore be beneficial for both the patient and the clinician.

The second part of this thesis consists of relevant clinical findings concerning the therapeutic options in this complex population. Over the past few years, various AEDs with new modes of action were introduced, but patients with ID and epilepsy are often excluded from the initial registration studies, as they are complicated for both practical and medical ethical reasons. We show that, although challenging, newer AEDs should not be avoided when facing problems in the current therapy, but the refractory nature of epilepsy should be part of counseling families so they are informed on realistic grounds.

Our last chapter shows that even when theoretically an AED should be avoided based on a specific etiology or diagnosis, withdrawal should by very closely monitored by an experienced neurologist or clinician who is aware of both the risks and benefits for this specific patient.

\section{Target groups}

The principal purpose of this thesis is to help colleagues working with people with ID and epilepsy to increase their knowledge with regard to evidence based assessments, investigations and ultimately also treatment choices in this complex population. We hope to encourage them to take an active role in the attempt to enhance the evidence-base of considerations and treatment choices.

With increasing the knowledge and awareness of the healthcare professional, the indirect target groups ofcourse are the patients and their relatives.

\section{Activities for innovation and implementation of knowledge}

All studies in this thesis are published or submitted as scientific manuscripts in peerreviewed international journals. Several findings have also been published in national peer-reviewed journals.

We participated in national and international congresses and symposia, also reaching out to non-academic healthcare professionals.

Additionally we included a large part of the outcomes in training programs and seminars for both doctors and nursing staff. 
Different findings of this thesis will be implemented and considered for the national epilepsy guideline by the "Nederlandse Vereniging voor Neurologie"(NVN).

Furthermore, this thesis resulted in a new national collaboration with the CCE ("Centrum voor Consultatie en Expertise"), bringing experts in the field of behavioral problems and epilepsy together in a multidisciplinary team which can be consulted throughout the whole of Netherlands.

Our international collaboration with the UK will be expanded to other countries, making more and more use of existing datasets. This enhances the opportunity to compare the different experiences from international centers in order to establish an international guideline that is, in line with the white paper ${ }^{9}$ focused on this specific population. International studies with a prospective design are part of the 'strategic nota' for 2020-2024 of our research-team. Since 2019 we are also part of the ILAE taskforce for people with ID and epilepsy.

Future plans include a website and/or app for patients and caregivers, which can be filled with results and alerts coming from different studies in the future, with which the pathway of considerations can be expanded. It is important to keep in mind that this pathway should be interpreted as a continuum rather than a definitive categorical structure, and the results of this thesis are just the primary pieces of the puzzle. 


\section{References}

1. Woittiez I, Ras M, Eggink E, et al. Hoe vaak komt een verstandelijke beperking voor? www.nationaalkompas.nl 2014b

2. Robertson J, Hatton C, Emerson E, Baines S. Prevalence of epilepsy among people with intellectual disabilities: A systematic review. Seizure. 2015;29:46-62.

3. Forsgren L, Beghi E, Oun A, Sillanpää M. The epidemiology of epilepsy in Europe - a systematic review. Eur J Neurol. 2005;12(4):245-253.

4. Royal College of Psychiatrists, College Report 206 Prescribing anti-epileptic drugs for people with epilepsy and intellectual disability [cited 2019 April 27 $7^{\text {th }}$. Available from: http://www.rcpsych.ac.uk/usefulresources/publications/collegereports/cr/cr206.aspx

5. Hamiwka L, Jones JE, Salpekar J, Caplan R. Child psychiatry. Epilepsy Behav. 2011;22(1):38-46.

6. Aldenkamp AP, Bodde N. Behaviour, cognition and epilepsy. Acta Neurol Scand Suppl. 2005;182:1925.

7. Lin JJ, Mula M, Hermann BP. Uncovering the neurobehavioural comorbidities of epilepsy over the lifespan. Lancet. 2012;380(9848):1180-1192.

8. Royal College of Psychiatrists. College Report 203 Management of epilepsy in adults with intellectual disability [cited 2019 July 29]. Available from: http://www.rcpsych.ac.uk/usefulresources/ publications/collegereports/cr/cr203.aspx

9. Kerr M, Linehan C, Thompson R, et al. A White Paper on the medical and social needs of people with epilepsy and intellectual disability: the Task Force on Intellectual Disabilities and Epilepsy of the International League Against Epilepsy. Epilepsia. 2014;55(12):1902-1906. 


\section{Dankwoord}

Jawel, het zit erop! De afgelopen jaren zijn voorbij gevlogen, op meerdere fronten. Mijn wetenschappelijke inspanningen liggen hier voor $u$ in dit mooie boekje. Maar dat was er nooit geweest zonder hulp van velen, want wetenschap in je eentje is naast ineffectief ook erg ongezellig. Mijn dank gaat dus naar iedereen die op welke manier dan ook een bijdrage heeft geleverd aan het bereiken van deze bijzondere mijlpaal.

Allereerst mijn promotieteam: Prof.dr. Bert Aldenkamp, dr. Jurgen Schelhaas, dr. Jos Hendriksen, drs. Francis Tan en mijn wetenschappelijke steun en toeverlaat: dr. Jans van Ool.

Bert, jij was het rustpunt in alle promotie-hectiek. Jouw kennis en expertise op het gebied van onderzoek zorgden altijd voor overzicht en enthousiaste plannen. Jouw snelle analyses en daaruit volgende plannen hebben er voor gezorgd dat ik me nauwelijks zorgen heb gemaakt of me dit wel ging lukken.

Jurgen, jij stond aan de basis van het ontstaan van het onderwerp van dit proefschrift. Jouw woorden "doe alsjeblieft iets waar je heel blij van wordt" gaven me het vertrouwen dat mijn ervaringen uit de praktijk belangrijk genoeg waren om heel veel onderzoek naar te doen. Het enthousiasme waarmee mijn ideeën werden ontvangen werkte aanstekelijk. Wat baalde ik dat je vervolgens bijna aan de andere kant van Nederland ging werken, gelukkig blijkt inmiddels dat afstand maar een relatief begrip is. Er volgen vast nog vele mooie projecten in de toekomst.

Jos, samen met Jurgen vormde jij een vaste basis voor mij en Jans. Je zorgde voor een stimulerend klimaat, en wist vaak met weinig woorden precies de kern te raken. De overwegingen vanuit jouw vakgebied waren een aanvulling op zowel mijn wetenschappelijke werk, als ook in mijn klinisch werk als AVG.

Francis, zonder jou was er helemaal geen onderzoek geweest. Vanaf het eerste moment dat ik 10 jaar geleden bij Kempenhaeghe binnenliep als beginnende dokter heb je me gestimuleerd om te groeien. Je bood me daar op verschillende manier alle kansen toe, waar ik je eeuwig dankbaar voor ben. Door jou wordt mijn drang naar kennis, kunde en onderwijs enigszins in goede banen geleid (en ja, ik weet dat ik soms lastig te sturen ben..). Je gunt me de vrijheid (soms na enige overtuigende betogen) om mezelf te zijn als dokter, wetenschapper en mens.

Jans, ik had dit nooit in $m^{\prime} n$ eentje kunnen (willen) doen. Jij vulde me op wetenschappelijk gebied perfect aan, en zorgde dat ik me niet verloor in al $m^{\prime} n$ enthousiasme. Af en toe moest je me weer even met beide benen op de (wetenschappelijke) grond zetten als ik weer eens iets verzonnen had dat echt niet 
met statistiek ondersteund kon worden. Wat was ik trots in januari toen jij jouw deel al mocht verdedigen, en ik voel me een stuk rustiger wetende dat jij letterlijk achter me staat tijdens mijn eigen verdediging. De komende jaren gaan vast nog heel veel mooie dingen brengen, werkgerelateerd en ver daar buiten $(;)$

Leden van de beoordelingscommissie; Prof.dr. R.J. van Oostenbrugge, Prof.dr. F.J.M. Feron, Prof.dr. L. Lagae, Dr. D.A.M. Maes-Festen en Dr. M.C. Marcelis, dank voor het lezen en beoordelen van mijn gehele proefschrift. Ik ben vereerd dat de gehele commissie tijd vrij heeft kunnen en willen maken om ook bij mijn verdediging in Maastricht aanwezig te kunnen zijn.

Zonder deelnemers uiteraard ook geen resultaten, ik wil dus graag alle bewoners van Kempenhaeghe, hun begeleiders en vertegenwoordigers bedanken voor hun enthousiasme, geduld, tijd en bemoedigende woorden. Wonen met begeleiding vanuit een expertisecentrum wil zeggen dat je vaak de vraag krijgt "wil je meedoen aan dit of dat onderzoek", en het feit dat we zoveel mensen bereid hebben kunnen vinden om mee te willen doen toont hoe betrokken jullie zijn. Door jullie kunnen we in de toekomst de zorg voor alle mensen met een verstandelijke beperking en epilepsie, ongeacht waar ze wonen, verbeteren. Zonder jullie zouden wij als dokters nooit de juiste hulp kunnen bieden.

Voor alle studies in dit boekje heb ik met heel veel verschillende mensen mogen samenwerken, zowel nationaal als internationaal. Mijn dank gaat uit naar alle coauteurs die een bijdrage hebben geleverd aan de verbetering van de kwaliteit van de publicaties; jullie kritische overwegingen, nieuwe inzichten, expertise en zelfs spellingscontroles waren onmisbaar.

Al die naaste collega's binnen (of inmiddels buiten) Kempenhaeghe die me op verschillende manieren de afgelopen jaren hebben gesteund met woorden, kennis, overnemen van klinisch werk of koppen koffie. Naomi, Sjef, Caroline, Ruby, Twan, Anneke, Irma \& Monique; ookal werken jullie niet meer allemaal binnen Kempenhaeghe, de afgelopen jaren waren zonder jullie nooit gelukt. Ik kon altijd op jullie rekenen als er weer eens een keer ondergedoken moest worden voor klinisch werk, zodat die laatste revisie toch net voor de deadline weer verstuurd kon worden. Daarnaast ook ons "kinderteam"; Judith, Marloes, Willeke en Hilde. Het geeft veel rust met zulke leuke collega's om je heen :- En tot die laatste categorie behoren uiteraard ook Inge, Richard, Marian. Jullie inspireren me allemaal op je eigen manier. Al die lieve collega's maken dat ik iedere dag met veel plezier weer naar m'n werk ga. Ik weet dat 
ik gevaar loop met opsommen van mensen..want de kans is groot dat ik iemand vergeet (zeker ook vanwege de postnatale toestand waarin ik momenteel dit dankwoord aan het typen ben): dus mis je jezelf.. wees niet teleurgesteld.. het liefst zou ik hier iedereen persoonlijk vernoemen aangezien er wat mij betreft geen 'rangorde' oid is, maar dan wordt het zo'n dik boekje ;)

Lieve vriendinnetjes; Mar, Mo, Ils, Rie, Lies \& Inge. Vol dromen begonnen we 22 jaar geleden (mijn hemel..wat worden we oud..) aan onze basis. Er zijn er maar weinig die me zo goed kennen als jullie.. het is niet voor niets dat jullie het over 'druk op Fran d'r manier' hebben ipv je teveel zorgen over me te maken. Al was daar helaas de afgelopen jaren wel af en toe aanleiding toe.. dank dat jullie er altijd voor me zijn, ook als ik er niet om vraag. Ik voel me een rijk persoon met zulke lieve en zorgzame mensen in m'n buurt.

En als ik het over dat laatste heb..dan kan ik nog een naam niet vergeten: Els (of Miek..ik zal proberen me op termijn aan te passen aan de meerderheid ;) ) dank voor al je wijze woorden, en dansjes uiteraard de afgelopen jaren.

Ook de 'Ladies' verdienen hier een plekje; Max, Berry, Dragan, Roderick, Guido, Maurice, Frank.. dank dat jullie er voor Arjen en mij zijn, jullie kunnen als geen ander voor de nodige ontspanning zorgen, zeker wanneer mijn hoofd (of dat van Arjen) weer eens overliep met ideeën of to-do-lijstjes.

Ik las wel eens ergens; "it takes a village to raise a scientist".. en ik vrees in mijn geval dat daar nog wel wat meer voor nodig was. Bedankt lieve (schoon)familie dat we met alles bij jullie terecht kunnen.

Irena \& Leandra, wat ben ik een trotse zus.. we zijn alle 3 zo anders, maar juist dat maakt ons zo bijzonder. En wat moeten jullie vroeger moe geworden zijn van al mijn wilde ideeën waar ik jullie tijdens de uitvoering uiteraard bij nodig had. Ik beloof plechtig de komende 50 jaar wat rustiger te worden, ik zal het proberen in ieder geval.., ;)

Papa en mama, het is vast niet altijd makkelijk geweest een dochter die het woord 'geduld' en 'rustig' niet in haar standaard pakketje had zitten. Maar wat ben ik jullie dankbaar voor alle motiverende woorden, het geduld en vertrouwen dat jullie me de afgelopen 34 jaar gegeven hebben. Het maakt me tot de volwassen vrouw (en moeder) die ik nu ben.

En dan tot slot..mijn eigen mooie gezin. Arjen, we zijn samen volwassen geworden..waar een glas goldstrike 18 jaar geleden al niet goed voor kan zijn :) 
Ik heb er geen woorden voor om te omschrijven hoe ik me voel als ik jou met onze 2 mini's zie zitten. En ook al was dit hele promotie-proces soms abacadabra voor je, je gaf me altijd het gevoel dat ik het kon. Dank, dank voor alles.. jullie zijn het meest dierbare wat ik bezit ()

Jenthe, Fabe.. dit boekje heeft helaas maar weinig plaatjes. Als jullie straks wat ouder zijn dan zal ik met alle plezier je uitleggen waarom mama dit gemaakt heeft. Jenthe, bedankt voor je mooie tekeningen, ik ben enorm trots op de kaft. En Fabe.. jouw komst was de beste deadline van ze allemaal! 


\section{Curriculum vitae}

Francesca Monica Snoeijen-Schouwenaars werd op 10 april 1985 geboren in Eindhoven. In 2003 behaalde ze haar Gymnasium diploma aan het Strabrecht College in Geldrop. Aansluitend startte zij de opleiding Geneeskunde aan de universiteit Maastricht. Ze liep coschappen in Aruba en Zuid-Afrika. Ook deed ze tijdens haar opleiding, naast haar werk bij de Albert Heijn en in een dorpscafé, vakantiewerk als woonbegeleider voor mensen met een verstandelijke beperking en epilepsie binnen Kempenhaeghe. Hier

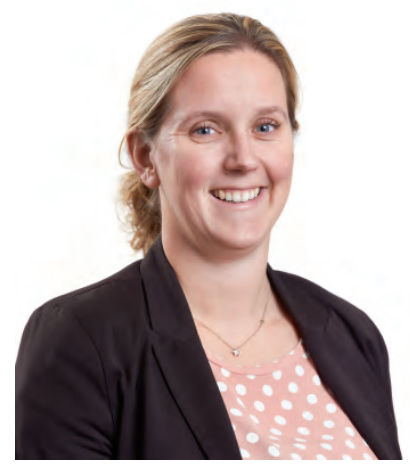
begon haar interesse voor deze specifieke doelgroep. Ze besloot haar laatste twee stages binnen dit expertisecentrum uit te voeren en dit was ook haar eerste kennismaking met wetenschappelijk onderzoek. Haar eerste onderzoek voerde ze uit naar de botkwaliteit van mensen met epilepsie en een verstandelijke beperking.

Na haar geneeskunde studie startte ze in 2009 als ANIOS Spoedeisende hulp / Intensive Care in het St.Anna ziekenhuis in Geldrop. In 2010 begon zij aan de specialisatie opleiding tot arts voor verstandelijk gehandicapten (AVG) aan de Erasmus Universiteit in Rotterdam. Tijdens deze opleiding werkte zij als AIOS bij stichting ORO in Deurne. Tijdens haar laatste jaar combineerde ze de opleiding met een parttime onderzoekscontract binnen Kempenhaeghe. Na afronding van de AVGopleiding begon zij in 2013 als AVG binnen Kempenhaeghe waar zij verantwoordelijk is voor de coördinatie van de medische zorg voor de volwassenen en kinderen die afhankelijk zijn van 24-uurs (epilepsie)-zorg. Ze is betrokken bij meerdere onderwijsprojecten en commissies. Vanaf 2015 combineert ze deze klinische zorg met haar promotietraject.

Francesca woont in Heeze, is getrouwd met Arjen en is de trotse moeder van Jenthe en Fabe.

Francesca Monica Snoeijen-Schouwenaars was born on April 10th 1985 in Eindhoven. In 2003 she graduated her secondary school education at the Strabrecht College in Geldrop. Subsequently she started Medical School in Maastricht University, and followed internships in Aruba and South-Africa. During her study, besides 
working at the Albert Heijn and a local pub, she had a job during the holidays in Kempenhaeghe as a caregiver for people with an intellectual disability (ID) and epilepsy. It was during this job this specific population attracted her interest.

She decided to perform her last year of Medical School in the expertise-centre of Kempenhaeghe, this was also her first acquaintance with scientific research. Her first study was in the field of bone-quality in people with ID and epilepsy.

After graduating Medical School in 2009 she worked as a resident in the emergency department and intensive care of the St.Anna Hospital in Geldrop. In 2010 she started her fellowship as a trainee physician for people with ID at the Erasmus University in Rotterdam, and she was working for stiching ORO in Deurne. During the last year of her fellowship she combined her clinical work with a research-position in Kempenhaeghe. After finishing her study in 2013, she started working as a full-time ID-physician at Kempenhaeghe, where she's responsible for the coordination of daily clinical care for both children and adults with ID and epilepsy. She's involved in many different teaching programs and committees. From 2015 forward, she combined this clinical care with her PdD-trajectory.

Francesca lives in Heeze, is married to Arjen and is the proud mom of Jenthe and Fabe. 


\section{List of publications}

\section{Peer-reviewed international journals}

- Snoeijen-Schouwenaars FM, van Ool JS, Tan IY, Aldenkamp AP, Schelhaas HJ, Hendriksen JGM. Mood, anxiety, and perceived quality of life in adults with epilepsy and intellectual disability. Acta Neurol Scand. 2019 Jun;139(6):519-25

- Snoeijen-Schouwenaars FM, van Ool JS, Verhoeven JS, van Mierlo P, Braakman $\mathrm{HMH}$, Smeets EE, et al. Diagnostic exome sequencing in 100 consecutive patients with both epilepsy and intellectual disability. Epilepsia. 2019;60(1):155-64.

- van Ool JS, Snoeijen-Schouwenaars FM, Tan IY, Schelhaas HJ, Aldenkamp AP, Hendriksen JGM. Classification of intellectual disability according to domains of adaptive functioning and between-domains discrepancy in adults with epilepsy. $J$ Intellect Disabil Res. 2019;63(1):40-8.

- Li X, Poschmann S, Chen Q, Fazeli W, Oundjian NJ, Snoeijen-Schouwenaars FM, et al. De novo BK channel variant causes epilepsy by affecting voltage gating but not Ca. Eur J Hum Genet. 2018;26(2):220-9.

- van Ool JS, Hurks PPM, Snoeijen-Schouwenaars FM, Tan IY, Schelhaas HJ, Klinkenberg S, et al. Accuracy of WISC-III and WAIS-IV short forms in patients with neurological disorders. Dev Neurorehabil. 2018;21(2):101-7.

- van Ool JS, Haenen Al, Snoeijen-Schouwenaars FM, Aldenkamp AP, Hendriksen JGM, Schelhaas $\mathrm{HJ}$, et al. Psychogenic nonepileptic seizures in adults with epilepsy and intellectual disability: A neglected area. Seizure. 2018;59:67-71.

- van Ool JS, Snoeijen-Schouwenaars FM, Tan IY, Jurgen Schelhaas H, Aldenkamp AP, Hendriksen JGM. Challenging behavior in adults with epilepsy and intellectual disability: An analysis of epilepsy characteristics. Epilepsy Behav. 2018;86:72-8.

- Snoeijen-Schouwenaars FM, Veendrick MJ, van Mierlo P, van Erp G, de Louw AJ, Kleine BU, et al. Carbamazepine and oxcarbazepine in adult patients with Dravet syndrome: Friend or foe? Seizure. 2015;29:114-8.

- Snoeijen-Schouwenaars FM, van Ool JS, Tan IY, Schelhaas HJ, Majoie MH. Evaluation of perampanel in patients with intellectual disability and epilepsy. Epilepsy Behav. 2017;66:64-7.

- Berkvens JJ, Veugen I, Veendrick-Meekes MJ, Snoeijen-Schouwenaars FM, Schelhaas $\mathrm{HJ}$, Willemsen $\mathrm{MH}$, et al. Autism and behavior in adult patients with Dravet syndrome (DS). Epilepsy Behav. 2015;47:11-6. 
- Snoeijen-Schouwenaars FM, van Deursen KC, Tan IY, Verschuure P, Majoie MH. Vitamin $D$ supplementation in children with epilepsy and intellectual disability. Pediatr Neurol. 2015;52(2):160-4.

- van Mierlo P, Snoeijen-Schouwenaars FM, Veendrick MJ, Tan IY, Willemsen MH, Schelhaas $\mathrm{HJ}$, et al. Letter: Recruitment of patients with both epilepsy and intellectual disability. Epilepsia. 2015;56(4):662-3.

- Peters CM, Schouwenaars FM, Haagsma E, Evenhuis HM, Echteld MA. Antibiotic prescribing and $\mathrm{C}$-reactive protein testing for pulmonary infections in patients with intellectual disabilities. Br J Gen Pract. 2013;63(610):e326-30.

- Beerhorst K, Schouwenaars FM, Tan IY, Aldenkamp AP. Epilepsy: fractures and the role of cumulative antiepileptic drug load. Acta Neurol Scand. 2012;125(1): 54-9.

\section{Other publications peer-reviewed national journals}

- Snoeijen-Schouwenaars, F.M., van Ool J. Neuropsychiatrische comorbiditeit bij volwassenen met epilepsie en verstandelijke beperking. TAVG 2018; 36 (4): 156-9.

- Majoie, H.J.M., Snoeijen-Schouwenaars, F.M. De verdiepingsstage epilepsie voor AVG's. TAVG 2018; 36 (4) 157-65.

- Snoeijen-Schouwenaars, F.M., van Ool J., Tan F., Wammes-van der Heijden, E. Schelhaas J., Majoie, M. Perampanel bij patiënten met een verstandelijke beperking. Epilepsie 2017; 15(1):5-7.

- Snoeijen-Schouwenaars, F.M., van Ool J. Literatuuronderzoek: Gedrags- en stemmingsproblematiek bij mensen met een verstandelijke beperking en epilepsie. Beschikbaar via: www.cce.nl

- van Mierlo P., Snoeijen-Schouwenaars F.M., Willemsen M, Wammes-van der Heijden E, Verhoeven J, Majoie M, Soubron J, Veendrick M, Tan F, Schelhaas J. GABAerge anti-epileptica bij patienten met een subcorticale band hetrotopie. Epilepsie 2016; 14(4):14-6.

- Sourbron J, Snoeijen-Schouwenaars F.M., Schelhaas J. Nieuwe classificatie voor corticale aanlegstoornissen. Epilepsie 2015; 13(4):6-10

- Peeters E, Schelhaas J, Snoeijen-Schouwenaars F.M. Kennis van epilepsie bij begeleiders van zorg voor mensen met een verstandelijke beperking. Epilepsie 2015; 13(3):12-4.

- Snoeijen-Schouwenaars F.M., Majoie M, Wammes-van der Heijden E, Arends J, Graveland G, Veendrick M. Nascholingsavond voor AVG-artsen: nieuwe antiepileptica. TAVG 2014; 32(1):54-6. 
- Veendrick M, Snoeijen-Schouwenaars F.M., Van Mierlo P, Van Erp G, Kleine B, Schelhaas J, Tan F. Carbamazepine afbouw bij volwassen patienten met het syndroom van Dravet. Correct volgens de literatuur maar pas op in de praktijk. Epilepsie 2014; 12(3):8-11. 
